\title{
Tuning DNA Compaction
}

\author{
Dissertation \\ zur Erlangung des Doktorgrades \\ der Mathematisch-Naturwissenschaftlichen Fakultäten \\ der Georg-August-Universität zu Göttingen
}

\author{
vorgelegt von \\ Rolf Dootz \\ aus Heidenheim
}

Göttingen, 30. Januar 2008 
D7

Referent:

Prof. Dr. T. Salditt

Koreferent:

Prof. Dr. S. Herminghaus

Tag der mündlichen Prüfung: $\quad$ 19.02.2008 


\section{ABSTRACT}

DNA compaction is the collapse of long DNA chains into well-organized condensates of complex, hierarchical nanostructure induced by the presence of cationic agents. Although much progress has been made in understanding underlying interaction mechanisms of in vivo DNA compaction, the interplay of the myriad compaction agents and their types of interactions with DNA still raise a wealth of unanswered, fundamental questions. In particular, the hierarchical organization of chromatin is widely unclear. There, the DNA is first wrapped around histone cores and the formed beads-on-a-string structure is successively shifted towards higher order forms of chromatin structure. The latter process involves linker histones as major antagonists.

Here, new results are presented that are derived from bio-mimetic investigations of the simplest possible DNA compaction model system containing only dendrimers, which can be viewed as uniformly charged cationic nanospheres, and unspecific, polydisperse DNA. Small angle X-ray (micro-)diffraction is employed as a principle method of analysis that accesses relevant molecular length scales. Targeting a quantitative understanding of compaction mechanisms, X-ray (micro-)diffraction measurements performed under laminar flow conditions in hydrodynamic focusing microfluidic devices provides microscale control of the self-assembly process. In addition, the method enables time-resolved access to structure formation in situ, in particular to transient intermediate states.

Utilizing the high level of control over dendrimer size and charge, DNA compaction is systematically tuned and analyzed in detail. Results show that dendrimers bridge the entire spectrum of biological condensation agents from small cations, such as spermine/spermidine encountered in viruses, to the much larger histone proteins found in eukaryotic cells. Despite its simplicity, the dendrimer/DNA system reproduces characteristic features of DNA compaction in vivo. In particular, PAMAM 6 dendrimers (having a size and charge comparable to histone core proteins) induce a complete wrapping of the DNA around the cation. As such, PAMAM 6/DNA entities are structurally artificial equivalents of nucleosome core particles. For cationic dendrimers having an intermediate size and charge, which is conveniently between that of small multivalent organic cations and larger histone-like proteins, an alternate route of DNA compaction aside from the established salt or macroion condensation is 
observed in microflow below the isoelectric point, where DNA is in excess of dendrimers.

In addition, the phenomenon of charge-induced dendrimer swelling has been experimentally quantified in detail over a wide range of generations. Results clearly show highly predictable, charge-induced changes of the dendrimer conformation and therefore eliminate the discrepancy between theory and experiments that previously existed in literature.

Besides artificial model-proteins, the interaction of linker histones H1 and DNA has been studied in microflow. The time-resolved access to struture formation dynamics clearly shows that the interaction of H1 with DNA is a two step process: an initial unspecific binding of $\mathrm{H} 1$ to DNA is followed by a rearrangement of molecules in the formed complexes. Results suggest that the conformational transition of $\mathrm{H} 1$ tails from their rather extended conformation, in aqueous solution, to their fully folded state, upon interaction with DNA, is most likely the motor of the conformational phase transition of H1/DNA assemblies.

Results obtained in this thesis are expected to have a direct bearing on the understanding of the hierarchical organization of chromatin in vivo. Underlying concepts and techniques may be generalized and used to experimentally address also other relevant protein/DNA systems. Moreover, the studied systems are of inherent importance to the field of biotechnology and are expected to contribute towards the design of new vectors for DNA gene delivery. 


\section{INHALT}

DNA-Kompaktion bezeichnet die Kondensation von langen DNA-Molekülen zu extrem dicht gepackten Aggregaten mit komplexer Nanostruktur. Induziert wird dieser Vorgang im Allgemeinen durch kationische Wechselwirkungspartner. Trotz der bereits erzielten großen Fortschritte auf dem Weg zu einem vollständigen Verständnis der zugrundeliegenden Wechselwirkungsmechanismen stellt das komplexe Wechselspiel der zahlreichen beteiligten Reaktionspartnern Wissenschafter noch immer vor eine Vielzahl unbeantworteter Fragen. Insbesondere der hierarchische Aufbau des Chromatins, bei dem die DNA zunächst auf Histon-Oktamere aufwickelt und anschließend - unter Beteiligung von Linker-Histonen - zu höheren Organisationsstufen überführt wird, ist weitgehend ungeklärt.

In der vorliegenden Arbeit werden biomimetische Untersuchungen des denkbar einfachsten Modellsystems für die DNA-Kompaktion vorgestellt. Dieses besteht nur aus Dendrimeren, die als gleichmäßig geladene, bis zu 10nm große, kationische Kugeln beschrieben werden können, und polydispersen DNA-Molekülen mit einer unspezifischen Basensequenz. Zur Untersuchung werden RöntgenDiffraktionsmessungen eingesetzt. Die Durchführung der Messungen im kontinuierlichen Fluss in Mikrofluidik-Bauteilen unter Ausnutzung hydrodynamischer Fokussierungseffekte gewährt einen kontrollierten und zeitaufgelösten Zugang zu verschiedenen Stadien des Selbstorganisationsprozesses. Dadurch können insbesondere transiente Zwischenstadien der Reaktion verfolgt und ein quantitatives Verständnis der Kompaktionsmechanismen erzielt werden. Der hohe Grad der Kontrolle über die Größe und die Ladung der Dendrimere ermöglicht es, verschiedene DNAKompaktionsszenarien gezielt einzustellen und im Detail zu analysieren.

Die Vielfalt der natürlich vorkommenden DNA-Kompaktionspartnern reicht von kleinen organischen Kationen wie Spermidin und Spermin in Viren bis hin zu den wesentlich größeren Histon-Oktameren in eukaryotischen Zellen. Die in dieser Arbeit vorgestellten Ergebnisse zeigen, dass sich Dendrimere ausgesprochen gut dazu eignen, dieses gesamte Spektrum zu reproduzieren. Trotz seiner starken Vereinfachung ist das Dendrimer/DNA-Modellsystem in der Lage, charakteristische Züge der natürlich auftretenden DNA-Kompaktion wiederzugeben. Besonders hervorzuheben ist dabei die Wechselwirkung von DNA mit PAMAM 6-Dendrimeren, die zu einem Aufwickeln der DNA um die in Bezug auf Ladung und Größe mit Histon-Oktameren vergleichbaren 
PAMAM 6 führt. Die gebildeten PAMAM 6/DNA-Einheiten zeigen eine erstaunliche Übereinstimmung in Größe und Gestalt mit nukleosomalen Kernpartikeln. Für Dendrimere mit einer mittleren Größe von ca. 3nm kann unter Verwendung von mikrofluidischen Methoden ein neuartiger DNA-Kompaktionsmechanismus unterhalb des isoelektrischen Punktes beobachtet werden. Dieser unterscheidet sich grundlegend von den bereits bekannten Salz- oder Makroion-induzierten DNAKondensationsformen.

Neben den Untersuchungen zur DNA-Kompaktion ist die Abhängigkeit der Dendrimerkonformation von der Ladung der Moleküle experimentell genau quantifiziert worden. Dabei sind vorhersagbare, ladungsabhängige Änderungen der Dendrimerkonformation beobachtet worden. Diese Beobachtungen beseitigen die Diskrepanz, die bisher in der Literatur zwischen theoretischen Vorhersagen und experimentellen Beobachtungen bestand.

Zusätzlich zu den künstlichen Modell-Proteinen wurde auch die Wechselwirkung von natürlich vorkommenden Linker-Histonen H1 mit DNA im Mikrofluss zeitaufgelöst untersucht. Die aufgezeichnete Röntgen-Streubilder belegen einen zweistufigen Wechselwirkungsmechanismus: Auf ein zunächst unspezifisches Anlagern der Proteine an die DNS folgt eine Reorganisation der Moleküle im gebildeten Komplex. Die erzielten Ergebnisse legen nahe, dass eine durch die DNA induzierte vollständige Faltung der endständigen H1-Proteinketten für den beobachteten Phasenübergang der H1/DNA-Komplexe verantwortlich ist.

Die in dieser Arbeit erzielten Ergebnisse leisten einen wichtigen Beitrag zum Verständnis des hierarchischen Aufbaus von Chromatin. Es ist davon auszugehen, dass die zugrundeliegenden Konzepte einen experimentellen Zugang zu weiteren relevanten Protein/DNA-Systeme gewähren werden. Darüber hinaus besitzen die hier untersuchten Systeme biotechnologische Bedeutung und es wird erwartet, dass sie zur Entwicklung zukünftiger Transfektionsvektoren beitragen werden. 


\section{CONTENTS}

1. INTRODUCTION...........................................................................11

2. METHODS OF ANALYSIS ................................................................19

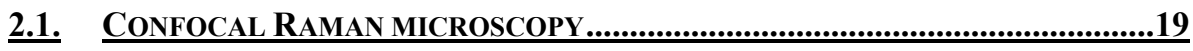

2.2. SMALL ANGLE X-RAY (MICRO)DIFFRACTION ...........................................22

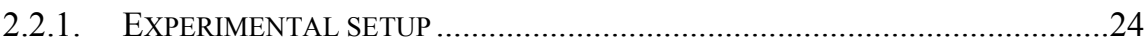

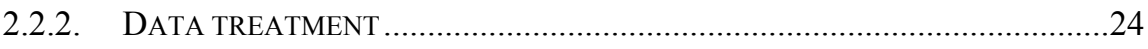

2.3. FINITE ELEMENT SIMULATIONS ……………........................................................27

3. PHYSICS OF THE MICROFLOW .................................................29

3.1. THE NAVIER-STOKES EQUATION .........................................................30

3.2. NO-SLIP BOUNDARY CONDITIONS AND CHANNEL VELOCITY PROFILES ........31

3.3. VISCOELASTIC MATERIALS IN MICROFLOW ……................................................32

3.4. DIFFUSION AND MIXING ..........................................................................33

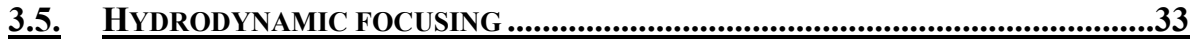

4. MICROFLUIDIC DEVICES............................................................38

4.1. MANUFACTURING MICROFLUIDIC DEVICES...................................................39

4.1.1. SOFT LITHOGRAPHY AND STANDARD PDMS DEVICES ................................39

4.1.2. KAPTON-STEEL-KAPTON DEVICES......................................................

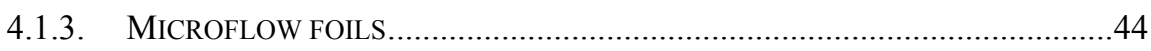

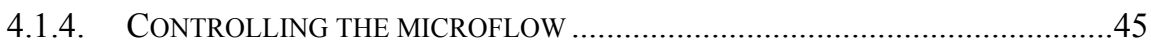

4.2. PROBING MICROFLOW FOILS ..............................................................................46

4.2.1. THE LIQUID CRYSTAL SYSTEM 8CB ....................................................

4.2.2. CHARACTERIZATION OF THE MICROCHANNELS........................................47

4.2.3. GEOMETRICALLY INDUCED BENDING OF 8CB SMECTIC A LAYERS ...........49

4.2.4. 8CB IN THE HYPERBOLIC CHANNEL SECTIONS ........................................50

4.3. CONCLUSIONS .......................................................................................................53

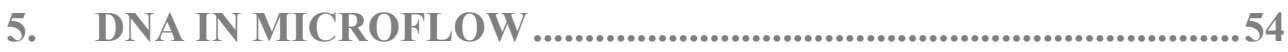




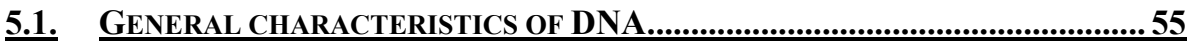

5.2. LIQUID-CRYSTALLINE PROPERTIES OF DNA IN SOLUTION ...........................56

5.2.1. X-RAY DIFFRACTION ON DNA SOLUTIONS ................................................ 58

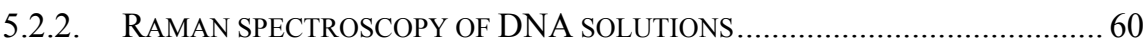

5.3. DNA IN MICROFLOW .....................................................................................62

5.3.1. INFLUENCE OF DNA SOLUTION VISCOSITY ON THE MICROFLOW ..................62

5.3.2. CONFOCAL RAMAN MICROSCOPY OF DNA IN MICROFLOW ……………............ 65

5.3.3. X-RAY MICRODIFFRACTION ANALYSIS OF DNA IN MICROFLOW................. 67

5.4. CONCLUSIONS ….....................................................................................69

6. DENDRIMERS:

CHARACTERIZING COMPACTION AGENTS................ 70

6.1. GENERAL STRUCTURE AND CHARACTERISTICS ............................................71

6.2. X-RAY DIFFRACTION OF DENDRIMER SOLUTIONS....................................... 74

6.3. GENERATION DEPENDENCE OF THE DENDRIMER CONFORMATION ……….... 79

6.4. PH DEPENDENCE OF DENDRIMER CONFORMATION....................................... 85

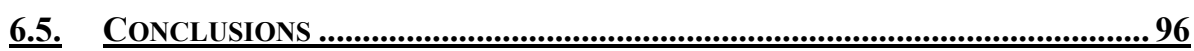

7. DNA COMPACTION:

DENDRIMERS OF INTERMEDIATE SIZE 97

7.1. CONFOCAL RAMAN IMAGING OF PPI 4 INDUCED DNA COMPACTION.........98

7.1.1. RAMAN SPECTRUM OF PPI 4/DNA COMPLEXES …………………......... 98

7.1.2. REAL-TIME MONITORING OF DNA COMPACTION IN MICROFLOW .............99

7.2. X-RAY MICRODIFFRACTION MEASUREMENTS OF PPI 4 INDUCED DNA

COMPACTION................................................................................................... 107

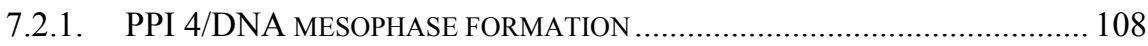

7.2.2. RESOLVING INITIAL STEPS OF MESOPHASE FORMATION............................ 112

7.2.3. COMPARISON WITH CONFOCAL RAMAN MEASUREMENTS ........................ 115

7.3. DNA COMPACTION BY DENDRIMERS OF INTERMEDIATE SIZE AND CHARGE

-A GENERALIZED DESCRIPTION ..................................................................116

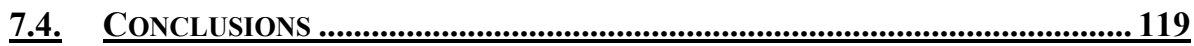

8. DNA COMPACTION:

DO DENDRIMERS MIMIC HISTONES?

8.1. INITIAL STATES OF PAMAM 6/DNA COMPLEX FORMATION .....................122

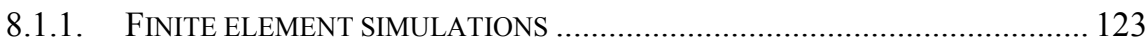

8.1.2. X-RAY MICRODIFFRACTION MEASUREMENTS IN FLOW ........................... 124

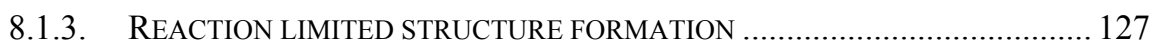

8.2. UNRAVELING THE INTERACTION MECHANISM............................................128 
8.2.1. FORM FACTOR OF PAMAM 6/DNA ENTITIES AT PH $=8.5$

8.2.2. DETERMINATION OF THE PAIR DISTANCE DISTRIBUTION FUNCTION .......129

8.2.3. AB INITIO MODELING OF PAMAM 6/DNA ENTITIES AT PH $=8.5 \ldots \ldots \ldots .133$

8.3. EXCEPTIONAL STRUCTURE OF PAMAM 6/DNA COMPLEXES .................138

8.3.1. 2D AND 3D HEXAGONAL ORGANIZATION OF PAMAM 6/DNA

COMPLEXES .138

8.3.2. 2D TO 3D STRUCTURAL TRANSITION ..................................................... 141

8.3.3. EVOLUTION OF COMPLEX STRUCTURE WITH TIME .................................. 145

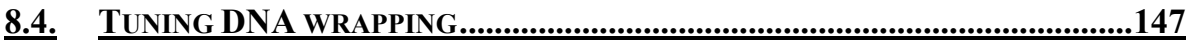

8.4.1. PH INDUCED PAMAM 6/DNA COMPLEX STRUCTURE TRANSITIONS .....147

8.4.2. FORM FACTOR OF PAMAM 6/DNA ENTITIES AT LOW PH ......................149

8.4.3. $\quad P(R)$ AND $R_{\mathrm{G}}$ OF PAMAM 6/DNA ENTITIES AT LOW PH .........................151

8.4.4. AB INITIO MODELING OF PAMAM 6/DNA ENTITIES AT LOW PH............153

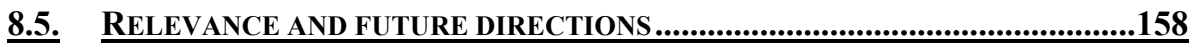

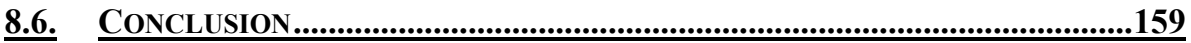

\section{DNA COMPACTION:}

LINKER-HISTONES H1

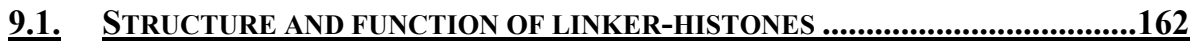

9.2. MONITORING H1 INDUCED DNA COMPACTION IN MICROFLOW ...............165

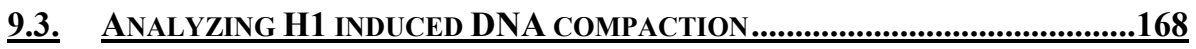

9.3.1. STRUCTURE OF H1/DNA MESOPHASES............................................... 168

9.3.2. MICRODOMAIN SIZES OF H1/DNA COMPLEXES ...................................175

9.3.3. LINKING H1 STRUCTURE TO FUNCTION .................................................176

9.4. DEMONSTRATION OF CERTAIN FUNDAMENTAL PRINCIPLES OF MICROFLUIDICS ENCOUNTERED FOR COMPLEX FLUIDS ..................................178

9.4.1. FLOW INDUCED ORIENTATION OF BIOMATERIALS ................................179

9.4.2. STRAIN INDUCED VARIATIONS IN MICRODOMAIN SIZE ............................181

9.4.3. BULK MEASUREMENTS OF H1/DNA COMPLEXES..................................185

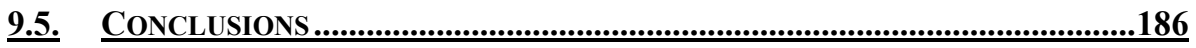

10. SUMMARY 187

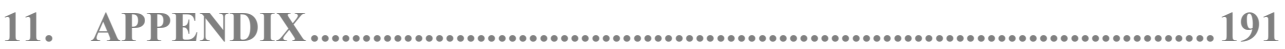

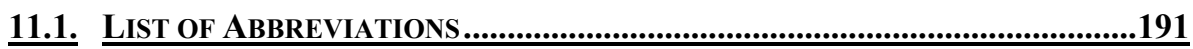

11.2. BIBLIOGRAPHY ........................................................................................197

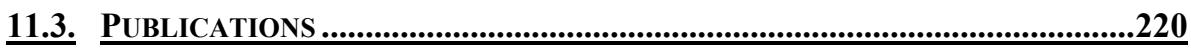




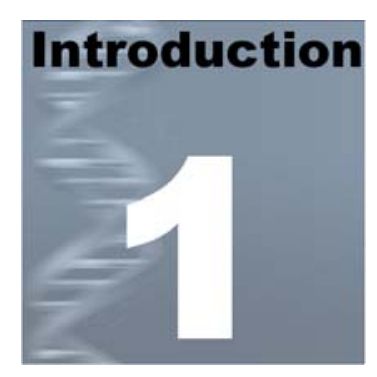

\section{INTRODUCTION}

DNA compaction is the collapse of long, extended DNA chains into compact condensates of complex nanostructure due to the presence of oppositely charged compacting agents. The associated reduction in DNA domain size is striking, as are the different, compaction agent dependent morphologies of the condensates. Therefore, the phenomenon of DNA compaction has drawn considerable attention.

From a more physical point of view, DNA compaction represents a demanding problem of phase transition, liquid crystal behavior, and polyelectrolyte interactions. A profound knowledge of the DNA organizing factors and driving forces is needed to understand processes such as replication or transcription, which depend crucially on the DNA packing, and to gain deeper insights into mimicking of biologically relevant DNA condensates. In particular, controlling of the conformational behavior of DNA through a fine tune of physical parameters of condensation agents and solvents should lead to the creation of new DNA condensates with an adjustable biological activity of the DNA chain. From a biological perspective, DNA compaction represents a reversible and dynamic process by which genetic information is packed and protected. Sophisticated and reversible packing mechanisms in vivo create highly flexible and dynamic DNA condensates that enable fundamental processes such as replication or transcription. Moreover, controllable DNA compaction in vitro is a promising method to deliver DNA containing genes of therapeutic interest to target cells.

Genetic DNA is a highly negatively charged, double-stranded macromolecule. The unique character of DNA is illustrated by its dimensions: the diameter of the DNA cylinder is approximately $2 \mathrm{~nm}$, its persistence length is in the range of $30-100 \mathrm{~nm}$, and 
although its contour length can be up to several meters, DNA is packed in micrometer sized cell nuclei. Therefore, DNA represents a unique bridge between the nano- and microscale with enormous potential for hierarchical organization.

Major driving forces of cationic DNA compaction are electrostatic interactions. DNA compaction is generally induced by partially neutralizing the negative charge of DNA phosphate groups. A general feature is the fact that a charge neutralization of about $90 \%$ is indispensable for DNA condensation. ${ }^{1}$ When multivalent cations are added to a DNA solution, they exchange places with mono- and divalent counter-ions screening the DNA. Entropic gains derived upon release of bound counterions ${ }^{2-4}$ and the associated reorganization of water molecules surrounding the DNA surface ${ }^{5}$ provide a second energetic contribution to the free energy of the DNA-cation system, which has been shown experimentally ${ }^{6}$ and theoretically ${ }^{7}$ to govern DNA compaction. When the multivalent ion concentration is raised above a certain threshold, a rapid aggregation of DNA segments into bundles results, which precipitate from solution. ${ }^{8}$ Remaining interactions between neutral polyplexes will generally be attractive and promote further aggregation into bigger clusters.

The structure of DNA condensates is regulated by the correlation between compaction agent dimensions and effective charge, on the one hand, and DNA charge density and chain rigidity, on the other hand. Two distinct mechanisms of DNA interaction with nanoscale structures can be found upon DNA compaction: DNA aggregates small and/or less charged compaction agents along its chain (Figure 1-1a) or it freely adsorbs on large, highly charged compaction agents (Figure 1-1b). The latter mechanism includes the possibility of DNA wrapping around nanoscale objects much smaller than its own persistence length and successive organization into higher-order structures. Accordingly, both scenarios of DNA compaction are clearly distinguishable in nature: compaction of DNA by small multivalent cations and compaction of DNA by interaction with proteins possessing a 3D structure. ${ }^{9,10}$

The first type of DNA compaction - shown in Figure 1-1a - is found in viruses and certain bacteria. In viruses, the compaction of double-stranded DNA is assisted by multi-cationic ions - in particular, small polyamines like spermidine and spermine that reduce the repulsion between DNA segments. This leads to the formation of extremely dense bundles with hexagonal symmetry and high DNA concentration. ${ }^{11-13}$ In bacteria suffering from potentially lethal conditions, DNA compaction by a mainly negatively charged DNA-binding protein named Dps (DNA binding protein from starved cells) is mediated via ion bridges forming stable, long-range ordered Dps-DNA columnar mesophases. ${ }^{14,15}$ 

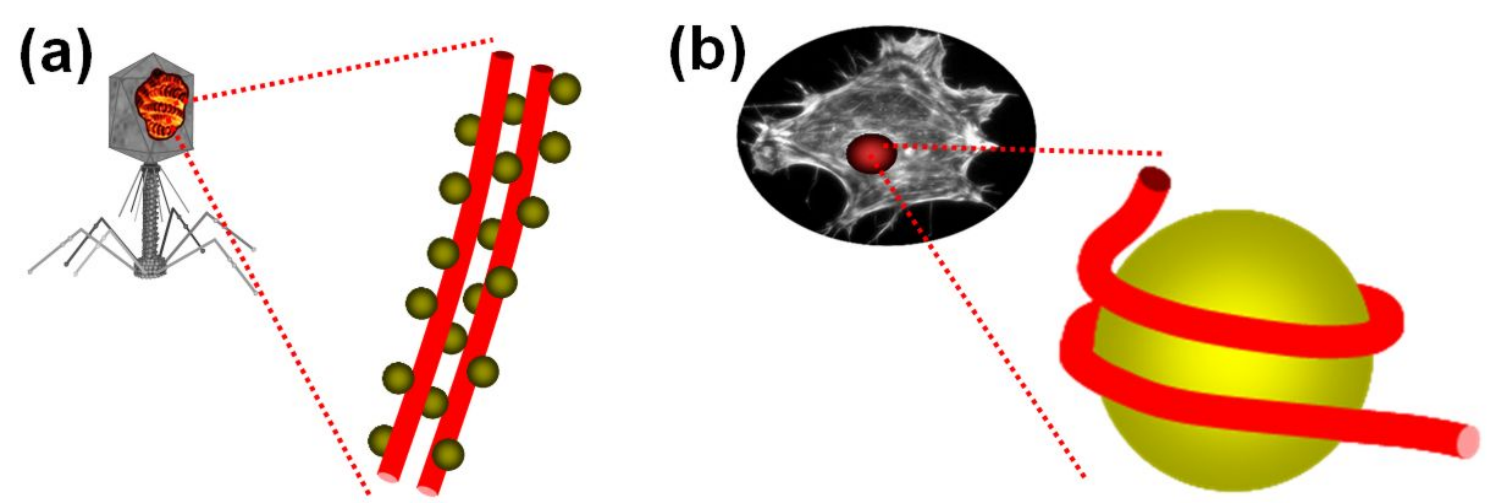

Figure 1-1: (a) In viruses, small compaction agents like spermidine and spermine aggregate along DNA strands. (b) In eukaryotic nuclei, the DNA is wrapped around histone proteins.

The second type of DNA compaction (Figure 1-1b) is manifested in bacteria ${ }^{14,16-18}$ and eukaryotic cells, ${ }^{9,} 19,20$ where the interaction of DNA with a multitude of proteins plays the dominant role. This results in multi-component, supercoiled structures of the DNA condensates. An exquisite phenomenon is the multi-hierarchical packing in eukaryotic cells. This involves local wrapping of short stretches of DNA around a highly basic, octameric histone protein core in order to form nucleosomal core particles, which are connected by a variable stretch of linker-DNA. ${ }^{21-25}$ Successive folding of the resulting "beads-on-a-string" structure into increasingly compacted filaments leads to well defined higher order structures (Figure 1-2). ${ }^{9,19,20}$ In this context, binding of linkerhistones to the linker-DNA plays a prominent role, facilitating the shift of chromatin structure towards more condensed, higher order forms (i.e. the $30 \mathrm{~nm}$ chromatin fiber). ${ }^{24}$ Therefore, linker-histones are of central importance in genome organization and regulation. However, despite extensive studies most fundamental questions such as linker-histone location and functions are still unanswered. Most surprisingly, so far there are no small angle scattering studies available in literature probing linkerhistone/DNA interaction dynamics and structure formation on relevant molecular length scales.

It is important to notice that even in cells DNA compaction is not only promoted by DNA-binding proteins, but also by molecular crowding and by the presence of multicharged cations. Interactions between DNA and cationic polyamines (e.g. spermidine, spermine) similar to those in viruses are speculated to be essential for the organization of the genetic material. ${ }^{26-28}$ Although much progress has been made in understanding DNA compaction in vivo, the interplay of the myriad compaction agents and the manifold types of interactions involved still raise a wealth of important 
1. Introduction

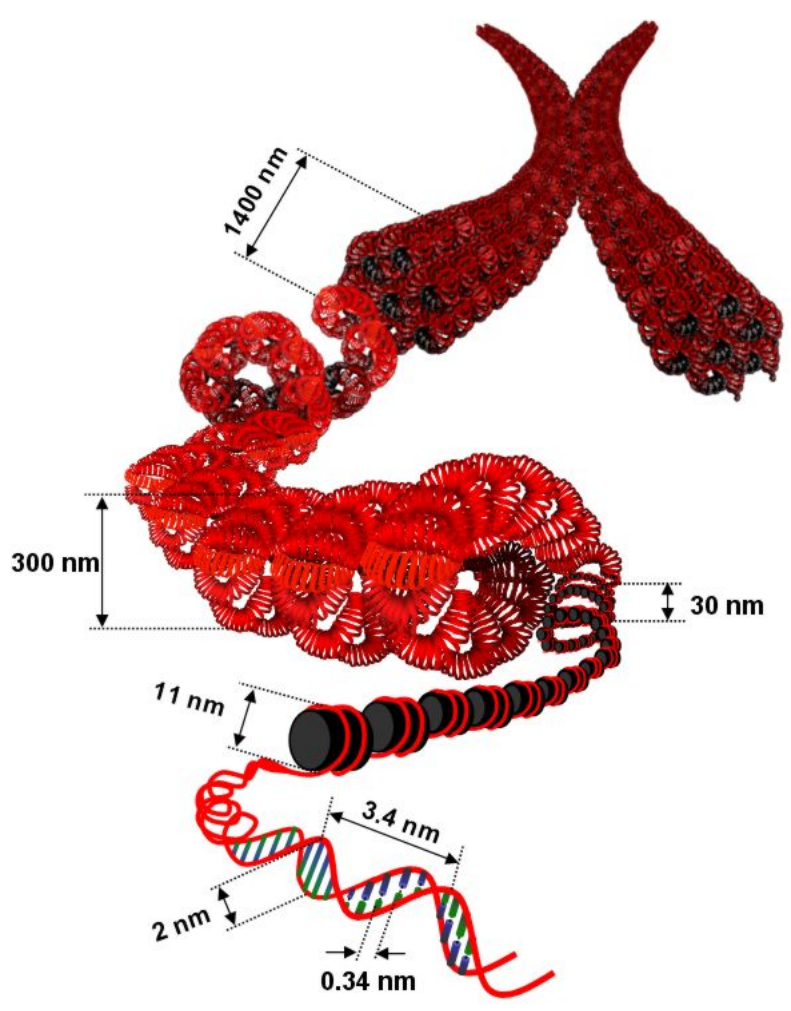

Figure 1-2: Multi-hierarchical self-organization of DNA in eukaryotic cells. Characteristic length scales are annotated.

questions. In particular, the chromosomal structure poses significant puzzles. Owing to the fact that DNA compaction in vivo is very challenging, one way to approach open problems is to mimic DNA compaction in vitro using purified, less complex systems. Benefiting from the fact that distinct active processes driven by energy consumption implied in DNA compaction in vivo - are eliminated, the physico-chemical interplay of DNA and compaction agent can be studied.

The properties of polycation/DNA condensates depend on various factors including the nature of ionic groups, the charge density, the proportion of opposite charges, the molecular weight of the macromolecules and the physicochemical environment (e.g. $\mathrm{pH}$, salt concentration). ${ }^{29,30}$ A broad number of nanoscale structures that compact DNA in vitro show similar characteristics to those observed in living organisms. Compacting agents with the ability to bridge the entire range of naturally occurring condensation agents from small cations, such as spermine/spermidine encountered in viruses, to the much larger histone proteins, in eukaryotic cells, are of special interest. Utilizing such agents should allow for investigations of the whole range of DNA compaction mechanisms - including like-charge attraction and formation of mesophases as well as electrostatically driven adsorption of DNA chain on oppositely charged compaction 


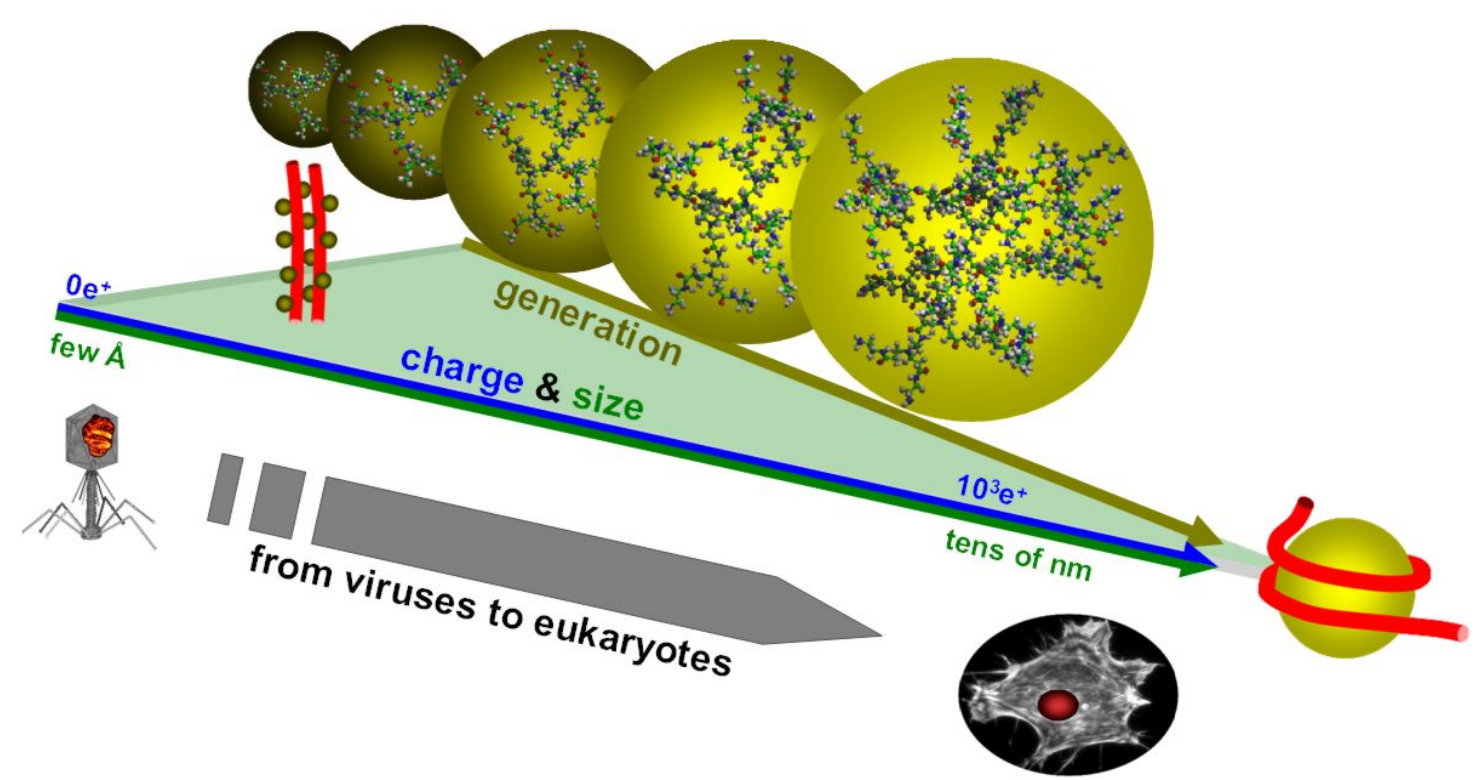

Figure 1-3: Tuning DNA compaction by varying dendrimer generation and therefore controlling the size and the charge of the compaction agent. This allows for covering the whole range of in vivo compaction scenarios from viruses to eukaryotes.

agents - by tuning only a small amount of controllable parameters. Moreover, the comparison of DNA compaction by histone proteins and model proteins may help to understand to what extent the consecutive hierarchical organization of chromatin including the wrapping of DNA around histones - happens due to energy consuming processes and how far it is dominated by pure electrostatic and electrodynamic interactions.

Recently, a revolutionary class of precisely engineered, spherical polycations ${ }^{31}$ called dendrimers ${ }^{32}$ have been synthesized, which are potentially the ideal molecules to enable DNA compaction studies. The systematic, layer-wise dendrimer build-up leads to extremely well defined molecules with a high structural and chemical homogeneity. ${ }^{33}$ Depending on the number of layers - termed generations -, dendrimer diameters can be tuned from a few angstroms up to tens of nanometers (Figure 1-3). ${ }^{34,35}$ Comprising protonable primary and tertiary amine groups, the dendrimer's charge can be controlled by variation of the solvent $\mathrm{pH}^{36,37}$ In addition, $\mathrm{pH}$-induced changes of dendrimer charges are generally expected to alter dendrimer conformation. However, the only experimental study on this topic reported no change of dendrimer size upon variation of $\mathrm{pH}$ value. ${ }^{38}$ Therefore, there is a discrepancy between theory and experiments making $\mathrm{pH}$-dependant conformational behavior a subject of considerable debate. ${ }^{38-43}$ 


\section{Introduction}

Besides potentially providing insights into in vivo DNA compaction mechanisms, there is a huge interest in utilizing dendrimer/DNA systems for drug delivery and as model systems for supramolecular aggregation. The resulting interest in dendrimer-induced DNA compaction stemming from both biology and physics has motivated many studies. However, the majority of these studies focus on the application of DNAdendrimer condensates for gene therapy disregarding fundamental, physical investigations of dendrimer/DNA interactions. ${ }^{44-52}$ So far, only two groups have performed (X-ray diffraction) studies to elucidate dendrimer/DNA complex formation. ${ }^{53,54}$ Moreover, only dendrimers of an intermediate size between that of lowmolecular DNA condensing agents (e.g. spermidine, spermine) and histones have been used for both studies. DNA chains in these complexes are found to organize into columnar mesophases with in-plane square or hexagonal symmetries depending upon the dendrimer generation number and dendrimer-to-DNA charge ratio. In addition, first microscopic observations indicate the existence of two different mechanisms of DNA compaction with dendrimers of low and high generations: ${ }^{55}$ Small dendrimers with only a few cationic groups collect on DNA chains, whereas for large dendrimers a wrapping of DNA chains around the dendrimers is speculated. However, beyond these first indications, no quantitative or unambiguous data are available and there is still poor understanding of the details of the interaction.

Due to their unique properties, dendrimers potentially provide the opportunity to fine tune their size and charge in a broad range by varying only few parameters such as generation number and $\mathrm{pH}$ of the solvent. Surprisingly, there do not seem to be any studies that utilize the huge variability in dendrimer size and charge to systematically tune DNA compaction and to analyze it in detail. The complexity of DNA compaction reactions arises from the involvement of many degrees of freedom, expected to be highly cooperative and involving conformational changes of the DNA as well as the compaction agent. However, no studies currently available consider these effects in the context of DNA compaction. Most of all, all studies analyzing dendrimer induced DNA compaction lack microscale control of the self-assembly process. None of them provides time-resolved access to structure formation in situ, in particular to transient intermediate states.

The aim of this thesis is to analyze DNA compaction dynamics and structure formation. Dendrimers of different size and charge, which act as model proteins, are used as compaction agents to systematically access the whole range of DNA compaction mechanisms. Since profound knowledge of the compaction agent properties is indispensable for understanding their interaction with DNA, dendrimers are also 


\section{Introduction}

analyzed in detail. In addition, the interaction and structure formation of linker-histones $\mathrm{H} 1$ and DNA is studied, in particular due to the important role of $\mathrm{H} 1$ for the organization of chromatin.

Experiments and results presented in this thesis are expected to provide fundamental insights into DNA compaction and organization in vivo, in particular due to the fact that special emphasis is given to time-resolved access to structure formation. To this end, DNA compaction is studied in hydrodynamic focusing microfluidic devices, targeting a quantitative understanding of compaction mechanisms. Aside advantages such as reduced sample volumes and shorter reaction and analysis times, microfluidics is a powerful tool for investigations of soft condensed matter and biological systems. ${ }^{56}$ The microfluidic setup is designed in a way that ensures a non-equilibrium ascent of reactant concentrations. Consequently, the study of different states of the reaction under controllable conditions during one measurement is possible by varying the observation position and the flow velocities in the microfluidic device. Due to the laminar flow conditions on the microscale, the compaction of DNA is diffusion controlled and allows for DNA compaction to occur in a highly defined way. Therefore, the formation of kinetically trapped structures is minimized and more compact condensates are formed in the microchannels.

The resulting progression of DNA compaction is detected online using spatially resolved X-ray microdiffraction. ${ }^{56-62}$ Small angle X-ray diffraction is a particularly useful tool for studies of DNA compaction, since it probes relevant length scales (on the order of $\mathrm{nm}$ ) and can illuminate multi-dimensional details of DNA condensates particularly when assemblies are aligned. ${ }^{63,64}$ The characterization of these materials, which are typically liquid-crystalline at ambient conditions, is significantly improved owing to a concurrent orientation during self-assembly processes. ${ }^{59,61}$

However, a major obstacle for performing X-ray diffraction measurements directly on a microfluidic chip is the lack of cheap and robust devices suitable for X-ray measurements (i.e. low X-ray scattering and absorption), which provide the ability of adapting the microchannel design according to the needs of each analyzed system. Therefore, it is necessary to first develop a straightforward and scalable method of fabricating long lifetime X-ray microdiffraction compatible microfluidic devices. ${ }^{63}$

In addition to small angle X-ray diffraction, confocal Raman microscopy is used to study molecular interactions of dendrimers and DNA, and to image structural formation in the microchannels. The combination of both the structural information, resulting from small angle X-ray scattering measurements, and the chemical sensitivity, obtained with Raman imaging, allows for detailed insights into the characteristics and the dynamics of self-assembled DNA condensates. 


\section{Introduction}

This thesis is organized as follows:

In chapter 2 the applied experimental techniques - Raman scattering and small angle X-ray scattering - are briefly discussed together with the details on instrumentation and data treatment. Basic aspects of the physics of the microflow are described in chapter 3. The manufacturing process of newly developed X-ray compatible microfluidic devices as well as a demonstration of their analytic power and geometric flexibility is given in chapter 4 . In chapter 5 , the liquid-crystalline properties of DNA in microflow are investigated. This is followed by a detailed analysis of the dendrimers in chapter 6 . Results concerning the dynamic assembly of DNA condensates by cationic dendrimers of different size are discussed in chapters 7 and 8 . Chapter 9 is devoted to the analysis of linker-histone H1/DNA interaction and structure formation. Moreover, the H1/DNA system is used to demonstrate basic principles of microfluidics of complex fluids and to show in detail the significant advantages for the characterization of such fluids arising from the combination of X-ray microdiffraction and microfluidics. In the final chapter 10 , the results are summarized. 
2. Methods

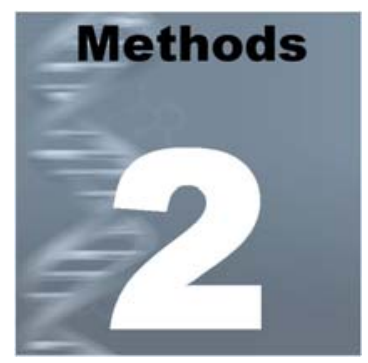

\section{Methods of Analysis}

In this chapter, the applied experimental techniques - Raman scattering and small angle X-ray scattering - as well as the utilized experimental setups are described in detail. Furthermore, basics of finite element simulations are briefly given.

\subsection{Confocal Raman microscopy}

The Raman effect is an inelastic scattering process, which has been discovered by C.V. Raman in $1928 .^{65}$ A classical electromagnetic field description of the Raman effect can be used to explain many of the important features of Raman band intensities. ${ }^{57}$ Exposed to an electric field,

$$
E=E_{0} \cos \left(\omega_{0} t\right)
$$

the electron cloud around a molecule is distorted and a dipole moment $p$ is induced:

$$
p=\alpha E
$$

The proportionality constant $\alpha$ is the polarizability of the molecule. The induced dipole scatters light at the frequency $\omega_{0}$ of the incident electromagnetic wave (Rayleigh scattering). However, Raman scattering occurs because a molecular vibration can 


\section{Methods}
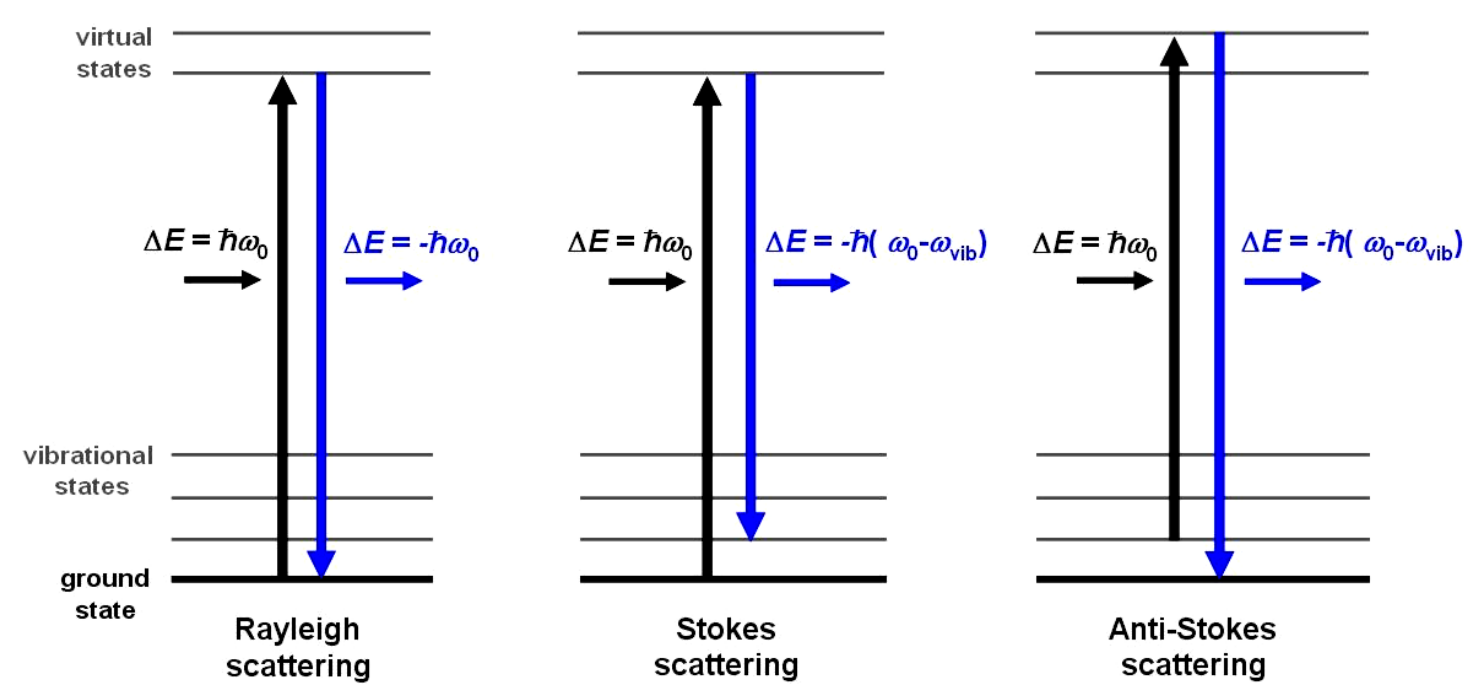

Figure 2-1: Rayleigh and Raman scattering.

change $\alpha$. The change is described by the polarizability derivative, $\partial \alpha / \partial r$, where $r$ is the normal coordinate of the vibration: ${ }^{66}$

$$
\alpha(r)=\alpha\left(r_{0}\right)+\frac{\partial \alpha}{\partial r}\left(r-r_{0}\right)+\ldots ; r=r_{0}+q \cos \left(\omega_{v i b} t\right) .
$$

Consistently, the dipole moment can be described by

$$
p(t)=\alpha\left(r_{0}\right) E_{0} \cos \left(\omega_{0} t\right)+\frac{\partial \alpha}{\partial r} E_{0} q\left[\cos \left(\omega_{0}-\omega_{v i b}\right) t+\cos \left(\omega_{0}+\omega_{v i b}\right) t\right]
$$

and the scattered light comprises Rayleigh and Raman contributions at frequencies of $\omega_{0}$ and $\left(\omega_{0} \pm \omega_{\mathrm{vib}}\right)$, respectively. According to the frequency of the Raman scattered light, one distinguishes between Stokes scattering, $\left(\omega_{0}-\omega_{\mathrm{vib}}\right)$, and Anti-Stokes scattering, $\left(\omega_{0}+\omega_{\text {vib }}\right)$.

One selection rule for a Raman-active vibration is given by ${ }^{66}$

$$
\partial \alpha / \partial r \neq 0
$$

This is analogous to the more familiar selection rule for an infrared-active vibration, which states that there must be a change in the permanent dipole moment during the vibration. According to the classical description given above, the scattering intensity is proportional to the frequency to the power of four. Therefore, Anti-Stokes scattering should be stronger than Stokes scattering. However, this is not observed.

In order to explain this phenomenon, a quantum mechanical approach to Raman scattering theory is needed. Quantum mechanically, the Raman effect can be described 


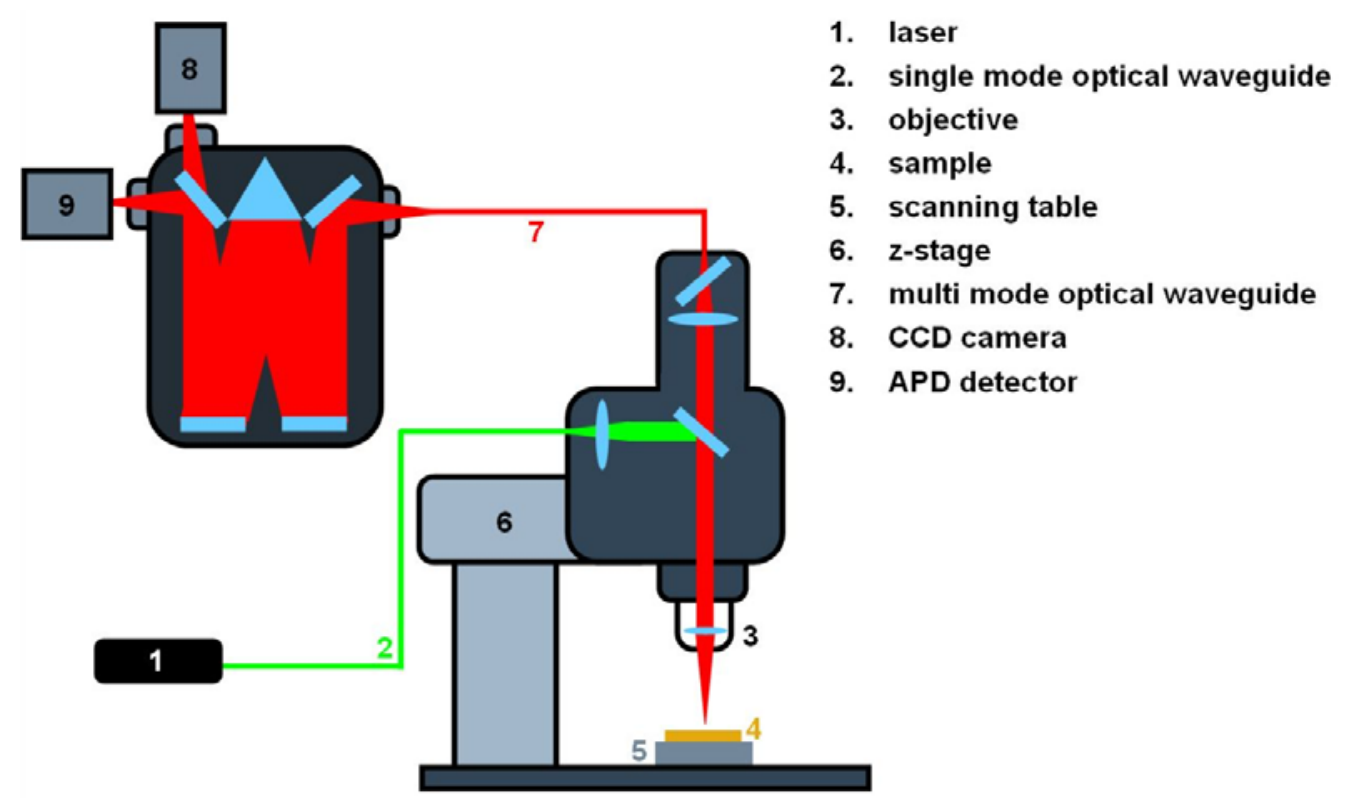

Figure 2-2: Schematic representation of the confocal Raman setup.

as a two photon process corresponding to the absorption and subsequent emission of a photon via an intermediate electron state (Figure 2-1). Vibrational and/or rotational energy $\hbar \omega_{0}$ can be exchanged. Thereby, light quanta are scattered, which have a scattered energy of $\hbar\left(\omega_{0} \pm \omega_{v i b}\right)$, giving rise to Raman lines. For Stokes scattering, the vibrational quantum number is changed by +1 whereas for Anti-Stokes scattering it is changed by -1 . Scattering intensities are proportional to the absolute frequency of initial states, which are Boltzmann distributed in thermal equilibrium. Accordingly, the higher intensity of Stokes scattering is due to higher absolute occupation of corresponding vibrational modes: ${ }^{57}$

$$
\frac{I_{\text {Anti-Stokes }}}{I_{\text {Stokes }}} \sim e^{-\frac{\hbar \omega_{v i b}}{k T}} .
$$

The frequency of scattered photons is specific to the molecular group from which the scattering occurs. Therefore, each molecule can be identified by its unique Raman spectrum.

Confocal Raman microscopy is a chemically sensitive method of imaging, which is independent of additional marker molecules and enables quantitative analysis and in situ detection. The method is suited to nucleic acids as well as for their complexes in aqueous solutions and in crystalline and non-crystalline solids. The applicability of Raman microscopy over a wide range of experimental conditions, such as solution temperature and composition, facilitates its use in the study of many biologically 


\section{Methods}

important structural transitions. ${ }^{67,68}$ However, biomaterials are sensitive to laser irradiation and can rapidly degrade when exposed to a laser beam for long periods of time. Measurements in microfluidic devices eradicate this concern, because while the laser beam remains at a fixed position, the material within the device continues to flow. Figure 2-2 shows a schematic representation of the confocal Raman microscope CRM200 manufactured by Witec (Ulm, Germany). The microscope is equipped with a piezzo scanning table, which can be moved $(200 \pm 0.004) \mu \mathrm{m}$ in $x$ - and $y$-, and $(20 \pm 0.001) \mu \mathrm{m}$ in $z$-direction. The laser beam (SGL-2200 laser, 532nm, $250 \mathrm{~mW}$, controlled by SUWTech LGD-2500, Shanghai Uniwave Technology) is focused on the sample in the microfluidic device using a diffraction limited spot size by an Olympus LMPlanFl 100x/0.80 objective. The measured signal is transmitted to a grating spectrograph via an optical fiber with a diameter of $50 \mu \mathrm{m}$. The end of this fiber is placed at the image plane of the microscope and used as a pinhole. The microscope provides the recording of spectra with an air-cooled CCD-chip (1340x1000 pixel) behind a grating spectrograph with a 600 lines per $\mathrm{mm}$ grating and a resolution of $6 \mathrm{~cm}^{-1}$. Additionally, high resolution 2D-Raman images of the sample at fixed wave numbers can be recorded by laterally $(x-y)$ and vertically $(x-z, y-z)$ scanning the sample through the excitation spot using the scanning table and detecting the Raman signal with a high quantum yield avalanche photodiode detector (APD). Recording of confocal Raman spectra and 2D scans is done using the programs ScanControl and ImageControl (WiTec GmbH, Ulm, Germany).

\subsection{Small angle $X$-ray (micro)diffraction}

Scattering of X-rays at small angles close to the primary beam yields information on sizes, shapes and the internal structure of particles. ${ }^{69}$ Apart from structural analysis of single particles, the method is suitable to study spatial correlations of particles. Covering relevant length scales from one to a few hundred nanometer, small-angle Xray scattering (SAXS) is a fundamental tool in the study of biological macromolecules. The major advantage of the method lies in its ability to provide structural information about disordered systems, i.e. about macromolecules in solution. Solution scattering studies are in particular important, given the fact that it is not possible to crystallize numerous macromolecules with high biological significance. Moreover, SAXS allows one to study the structure of native particles in near physiological environments and to analyze structural changes in response to variations of external conditions at relative low effort. ${ }^{69-71}$ 
However, due to the low information content of small angle scattering data from solutions in the absence of crystalline order, there is only a limited number of independent parameters that can in principle be extracted from such scattering data. According to Shannon's sampling theorem, ${ }^{72-74}$ the number of degrees of freedom associated with $I(q)$ on an interval $\left[q_{\min }, q_{\max }\right]$ is estimated as

$$
N_{S}=\frac{D_{\max }\left(q_{\max }-q_{\min }\right)}{\pi},
$$

with $D_{\max }$ representing the maximum particle diameter. Due to the fact that small angle scattering curves decay rapidly with $q$, they are reliably registered only at low resolution and, in practice, $N_{\mathrm{S}}$ does not exceed $10-15 .^{75}$ Therefore, small angle scattering is commonly considered to be not only a low-resolution but also a lowinformation technique. Here, the number of Shannon channels is $N_{\mathrm{S}} \approx 8$ (estimated with the program $\mathrm{GNOM}^{76}$ ). Fitting complex models, which contain a larger number of parameters, to recorded scattering data can not yield a stable solution without additional a priori information and results are expected to display a strong dependence on initial parameters. Accordingly, the model derived for describing the dendrimers in chapter 6.2 includes only 4 independent fit parameters.

Molecular sizes, shapes and inter-molecular interferences contribute to the scattering curves. SAXS experiments measure the Fourier transform of the electron density of objects in the sample. ${ }^{71}$ The electron density of biomolecular assemblies is a convolution of the electron density of the single scattering objects with the $2 \mathrm{D}$ or $3 \mathrm{D}$ lattice of delta-functions that define their arrangement in the complex. Thus, the scattering intensity $I(q)$ consists of Bragg peaks, determined by the structure factor $S(q)$, accounting for interference effects between particles, with peak heights modified by the smoothly varying form factor, i.e. the single particle scattering function, $F(q)$ :

$$
I(q) \sim N F(q) S(q) .
$$

$N$ is the number of particles. $q$ is the scattering vector defined by the irradiated wavelength $\lambda$ and the scattering angle $\theta$ according to the following equation:

$$
q=\frac{4 \pi \sin \theta}{\lambda} .
$$

Bragg peak heights and shapes are determined by lattice vibrations, defects, finite-size effects, and other distortions, whereas the peak positions determine the symmetry and dimensions of the lattice. ${ }^{71,77}$ 


\subsubsection{Experimental setup}

Small angle X-ray scattering (SAXS) measurements are conducted at the beam-line ID10b at the European Synchrotron Radiation Facility (ESRF, Grenoble, France). The ID10b beam-line is a multi-purpose, high-brilliance undulator beam-line for high resolution X-ray scattering and surface diffraction on solids and liquids. ${ }^{62}$ The sample is mounted onto the ID10b goniometer. Beryllium compound refractive lenses $(\mathrm{CRL})^{78}$ with a focal distance $f \approx 1.3 \mathrm{~m}$ are used to focus the synchrotron X-ray beam of $8 \mathrm{keV}$ $(\lambda=1.55 \AA)$ down to a spot of $d_{\mathrm{S}} \approx 20 \mu \mathrm{m}$ in diameter. Moving the microdevice along coordinates $x$, and $y$, the X-ray beam is positioned at the desired position. A CCD camera with fluorescent screen is used as a detector. Resulting 2D images of diffraction patterns cover a $q$-range of $0.25-3.50 \mathrm{~nm}^{-1}$. Due to the high-flux X-ray beam, short exposure times of 30-120s are possible.

In addition, experiments are performed using an in-house Bruker AXS Nanostar (Bruker AXS, Karlsruhe, Germany). This setup includes a rotating anode X-ray source for $\mathrm{Cu}-\mathrm{K}_{\alpha}$ radiation $(1.54 \AA$ ) at a generator power of $4.05 \mathrm{~kW}$. The $\mathrm{X}$-ray beam is adjusted to a size of $d_{\mathrm{N}} \approx 100 \mu \mathrm{m}$ or $400 \mu \mathrm{m}$ by a set of three pinholes. 2D scattering data are recorded using a virtually noise-free, real-time 2D Hi-Star detector with photon counting ability. Providing the opportunity of varying the sample-detector distance between $6-120 \mathrm{~cm}$, in-house a total $q$-range of $0.1-9.0 \mathrm{~nm}^{-1}$ is covered. This allows for resolving of feature sizes ranging from $0.7-62.7 \mathrm{~nm}$. Due to the lower adsorption of injected fluids compared to material of the channel walls, the in-house setup enables real space imaging of microfluidic devices via lateral and vertical X-ray absorption scanning with a step size down to $50 \mu \mathrm{m}$. This allows for precise selection of measurement positions. According to the significantly lower intensity of the in-house X-ray beam, exposure times of up to two hours per image are necessary. Performing measurements under continuous flow conditions is therefore indispensable to the retention of molecular integrity, especially when analyzing complex materials that can be easily destroyed due to the high X-ray energy (chapter 9.4.3). ${ }^{59-61}$

\subsubsection{Data treatment}

SAXS data were acquired from complex materials either inside of microfluidic devices or from samples loaded in thin quartz capillaries with a wall thickness of $0.01 \mathrm{~mm}$ and diameters varying between $1.3-1.6 \mathrm{~mm}$. All collected data are first corrected for detector sensitivity by using the Bruker recording software SAXS. Background intensities 

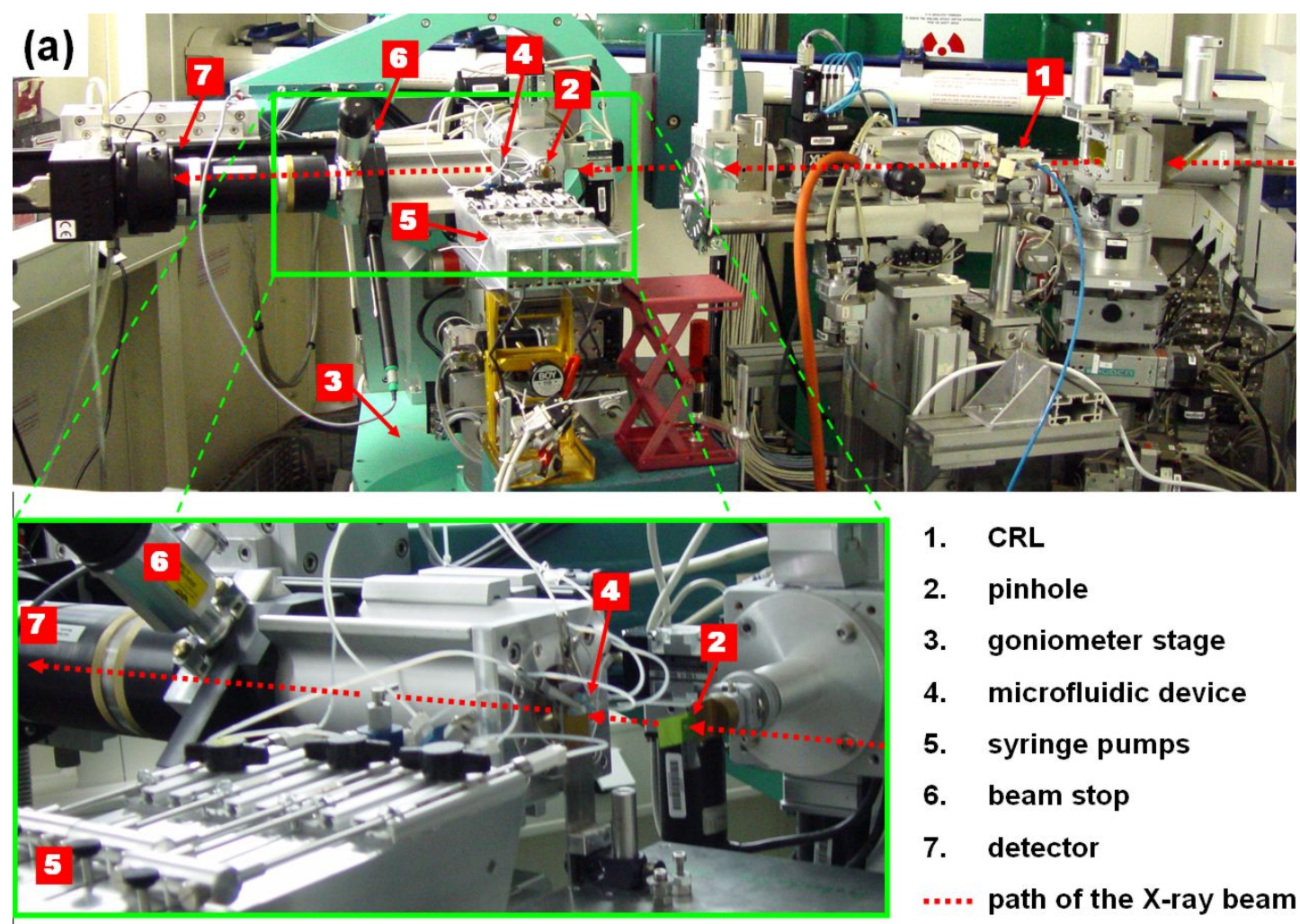

1. CRL

2. pinhole

3. goniometer stage

4. microfluidic device

5. syringe pumps

6. beam stop

7. detector

..... path of the X-ray beam
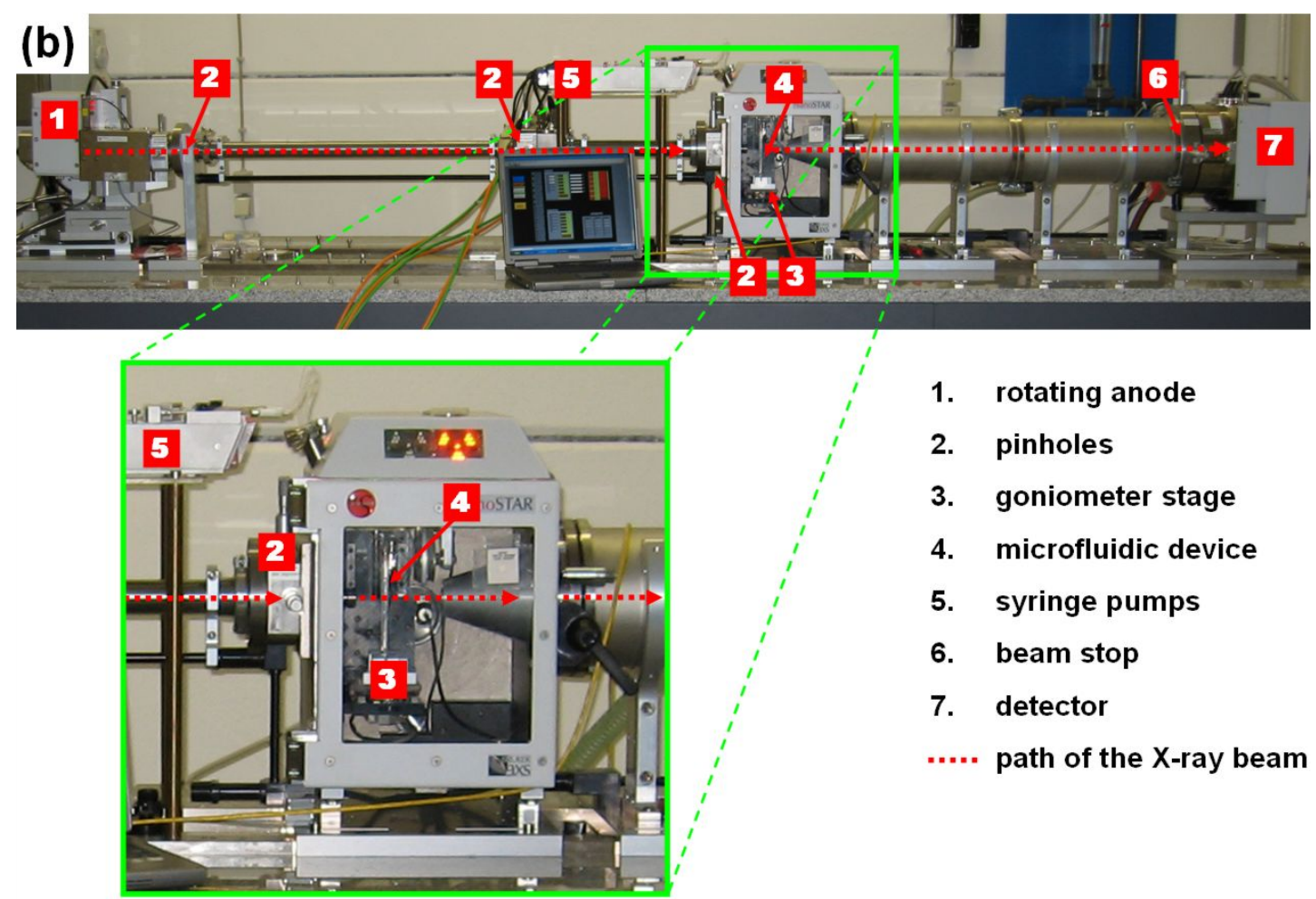

1. rotating anode

2. pinholes

3. goniometer stage

4. microfluidic device

5. syringe pumps

6. beam stop

7. detector

..... path of the X-ray beam

Figure 2-3: Experimental synchrotron beam-line (a) and in-house (b) setup. 

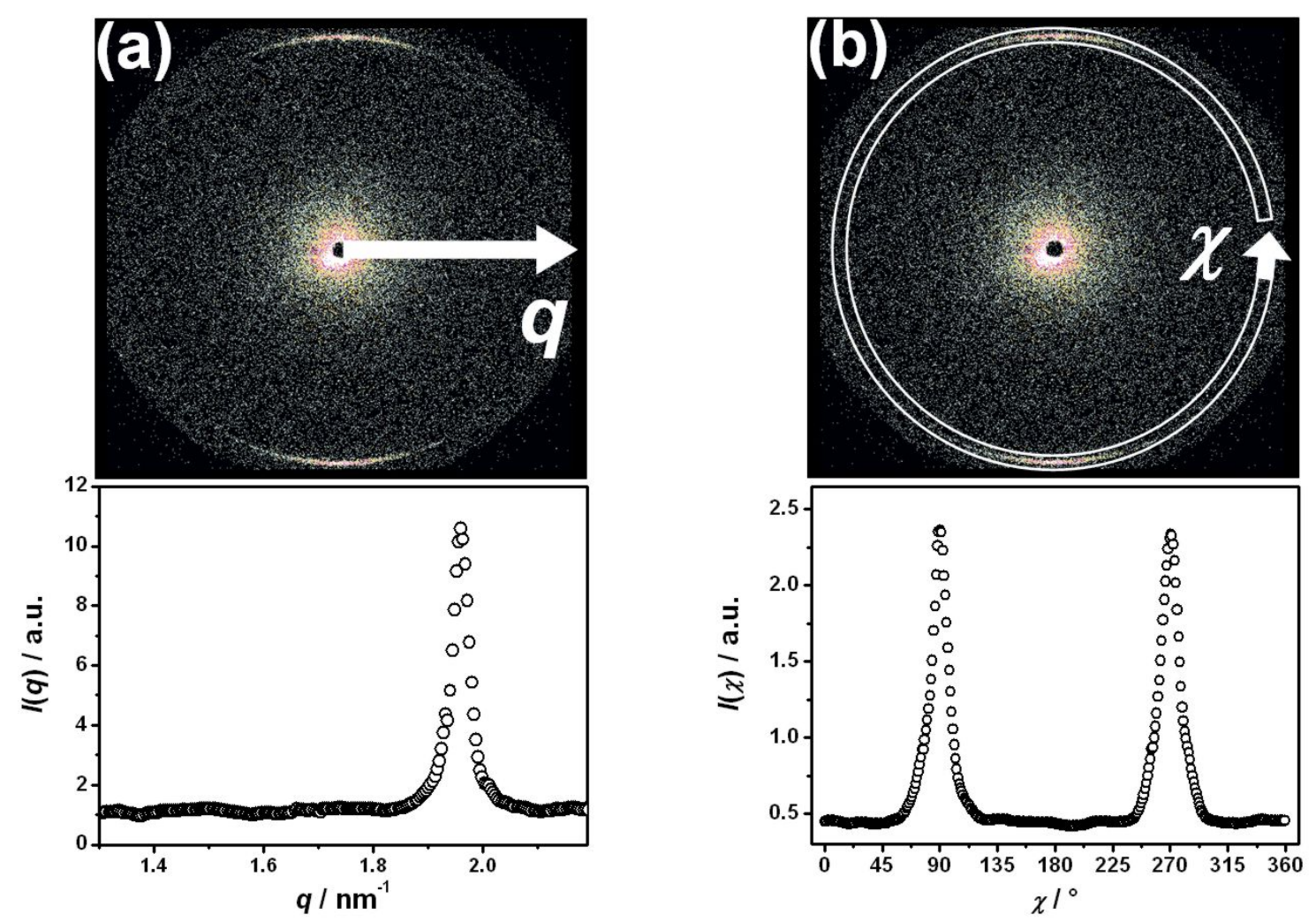

Figure 2-4: Converting 2D raw data images to $1 D$ intensity plots. (a) $I(q)$ and (b) $I_{q \max }(\chi)$.

caused by scattering from the X-ray windows of the detector vacuum chamber, glass capillaries or microchannel devices, the solvent, and the beam stop overspill are determined by separate reference measurements. In order to account for different capillary diameters and to determine absolute scattering intensities, each reference data is weighted by the ratio of overall scattering intensities $I_{\mathrm{GC}}$ (sample)/ $I_{\mathrm{GC}}$ (reference) before subtracting from the respective sample data. $I_{\mathrm{GC}}$ is acquired by inserting glassy carbon in the beam path at a fixed position closely after the sample and measuring for 100s.

A typical 2D X-ray image shows patterns of intensity $I(q, \chi)$ corresponding to features of the liquid-crystalline lattices that are probed (Figure 2-4). The real spacing $d$ of these features is inversely proportional to the momentum transfer

$$
q=4 \pi / \lambda \sin \theta
$$

where $\lambda$ is the X-ray wavelength and $2 \theta$ is the angle between incident and scattered radiation. A plot of intensity $I$ versus $q$ is obtained by azimuthally integrating the image over $360^{\circ}$ : 


$$
I(q)=\int_{0}^{2 \pi} I(q, \chi) d \chi
$$

The azimuthal distribution of the peak intensity $I_{\mathrm{qmax}}(\chi)$ in a fixed $q$ region $q_{\max } \pm \Delta q$ around a peak position $q_{\max }$ is also a quantity of interest, in particular because an alignment of materials occurs in microflow.

$$
I_{q_{\max }}(\chi)=\int_{q_{\max }-\Delta q / 2}^{q_{\max }+\Delta q / 2} I(q, \chi) d q
$$

Materials investigated in this thesis are not single-crystalline, and their 2D scattering images reflect a powder average from multiple orientations. This results in an intensity distribution along the pattern at fixed radial position $q$. In Figure 2-4, the extraction of data from a $2 \mathrm{D}$ raw data image is demonstrated and resulting plots of intensity $I$ versus $q$ and $I$ versus the azimuthal angle $\chi\left(\chi=0^{\circ}-360^{\circ}\right)$ along a fixed $q$ region, respectively, are shown. In addition, the full width at half maximum of a peak of $I(\chi), \Delta \chi$, serves for quantifying the extent of material orientation within microdevices.

For recording in-house X-ray data and for their evaluation, the program SAXS (Bruker $\mathrm{GmbH}$, Karlsruhe, Germany) is used. In addition, X-ray data processing is performed using the program Fit2D by Andy Hammersley. ${ }^{79}$ For the analysis of the interaction of PAMAM dendrimers generation 6 and DNA, presented in chapter 8.2, the programs GNOM $^{76}$, DAMMIN, ${ }^{80}$ and CRYSOL ${ }^{81}$ by Svergun et al. are used.

\subsection{Finite Element Simulations}

In this thesis, finite element simulations using the program Comsol Multiphysics 3.2 (Femlab GmbH, Göttingen, Germany) are performed for numerical solutions of differential equations describing the physics of the microflow in microchannel devices: the Navier-Stokes equation, which is coupled with diffusion equations for involved components (chapter 3).

The basic concept of the finite element method is to divide a structure into smaller elements of finite dimensions and simple shape (e.g. triangles), which are connected at a finite number of joints. The mathematical problem is locally adapted for each finite element in a way that equations of equilibrium for the global structure are obtained by combining the equilibrium equation of each element. Thereby, the continuity is ensured at each joint. Imposing boundary conditions, equations of equilibrium are solved to obtain required variables such as stress, strain, concentration distribution or velocity 


\section{Methods}

field. Thus, instead of solving the problem for the global structure in one operation, the problem is solved separately for the constituent elements.

Since numerous physical phenomena like viscosity effects and surface tensions have to be taken additionally into account, computational power is often precluding numerical calculations of the real 3D problem. However, exploiting the symmetry of the channel design enables to reduce the problem and therefore the computation time without affecting principle physical phenomena. ${ }^{82}$ 
3. Microfluidics

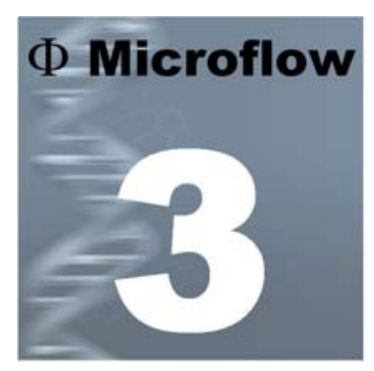

\section{PhySICS OF THE MICROFLOW}

The miniaturization, integration, and analysis of chemical and biological processes on the nanoliter-scale continue to drive remarkable progress in the fields of biotechnology, protein crystallization, and combinatorial chemistry. ${ }^{83-87}$ Aside from advantages such as reduced sample volumes and the possibility of high throughput and parallel operations, microfluidics is a powerful tool for fundamental investigations of soft condensed matter and biological systems. ${ }^{56}$ Allowing to manipulate single cells and even single macromolecules, there is great interest in using microfluidic systems for analytical tests. $^{58,83,88,89}$ However, with miniaturizing fluid transport systems to the microscale, there is a fundamental change in hydrodynamics that occurs. At these scales, inertial forces are virtually non-existent, surface tension plays an important role, diffusion governs mixing, and evaporation acts quickly on exposed liquid surfaces. In order to understand and work with microfluidics, one must first face the physical phenomena that dominate at microscale dimensions. 


\subsection{The Navier-Stokes equation}

The basic equation for the dynamics of a Newtonian fluid is the Navier-Stokes equation, $^{90}$

$$
\rho\left(\frac{\partial u}{\partial t}+(u \nabla) u\right)=-\nabla p+\eta \nabla^{2} u+f
$$

where $\rho$ is the fluid density, $u$ the fluid velocity, $p$ the pressure, and $\eta$ the fluid viscosity. Since fluids are continuum materials, the Navier-Stokes equation essentially represents the continuum version of Newton's law $F=m a$. Forces per unit volume on the right-hand side are balanced by inertial acceleration terms on the left-hand side. $f$ represents body force densities. ${ }^{90-92}$

To determine the relative effect of viscous and inertial forces, the dimensionless Reynolds number can be considered:

$$
\operatorname{Re}=\frac{\rho u l}{\eta}
$$

$l$ is a typical length scale of the observed system. On macroscopic dimensions, where turbulences are governing fluid behavior, Reynolds numbers of $10^{3}$ or higher are found. Owing to the small dimensions of microfluidic devices, Reynolds numbers reached for any of the experiments discussed in this thesis are all in the order of $\operatorname{Re}<1$. At such low Reynolds numbers, viscous forces overwhelm inertial forces and dominate fluid dynamics. Therefore, the left-hand side of the Navier-Stokes equation can be neglected, leaving the linear Stokes equation:

$$
\eta \nabla^{2} u+f=\nabla p
$$

The fluid flow is determined entirely by the pressure distribution, the incompressibility constraint,

$$
\nabla \times u=0,
$$

required from mass conservation,

$$
\partial \rho / \partial t+\nabla(\rho u)=0
$$

and boundary conditions. Contrary to the Navier-Stokes equation, the Stokes equation is symmetric in time. 

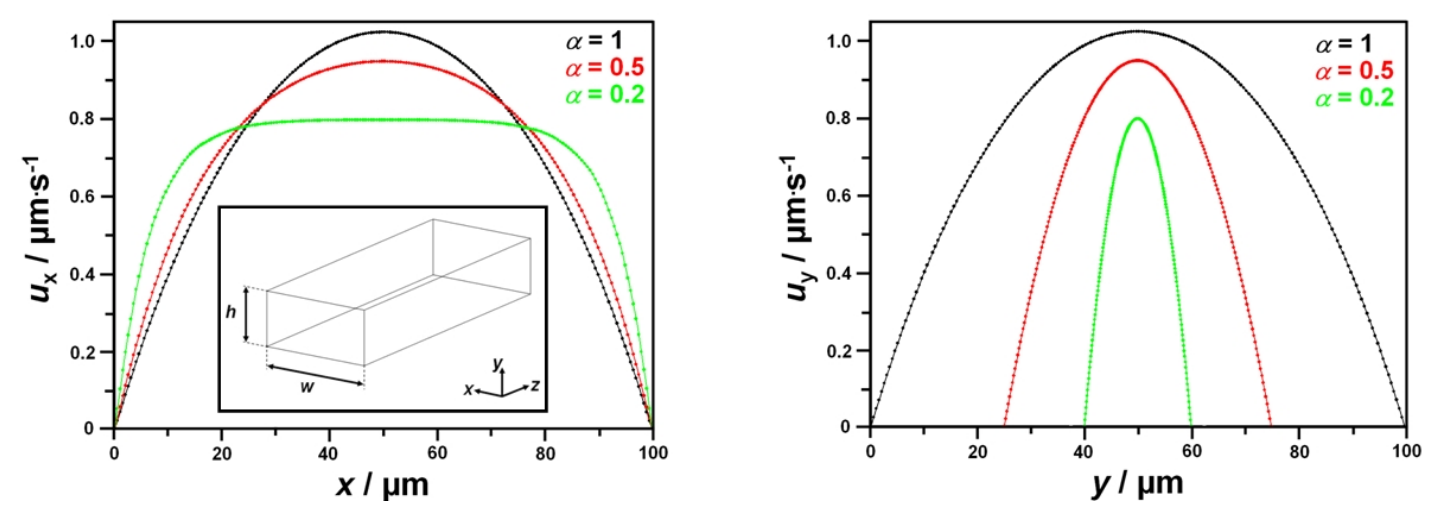

Figure 3-1: (a) Normalized velocity profiles along the wide (a) and the narrow (b) channel aspect obtained from finite element simulations.

\subsection{No-slip boundary conditions and channel velocity profiles}

The influence of boundary conditions is significant affecting in particular the relation between pressure gradients and volumetric flow rates, and the velocity profile perpendicular to the mean flow direction. In general, no-slip boundary conditions ( $u=0$ at channel walls) have been accepted not so much due to convincing theoretical background but rather due to apparent experimental success. ${ }^{91}$ However, recent measurements have shown evidence for partial slip of liquids at solid boundaries. ${ }^{93-97}$ The magnitude of the apparent slip length - the velocity profile extrapolates to zero at this distance inside the wall - measured seems to be highly sensitive to the nature of the surface, in particular to surface roughness and wettability. Using solely microchannels with hydrophilic walls in combination with aqueous solutions in this thesis, it is reasonable to assume no-slip boundary conditions. ${ }^{95,97}$ Typical microchannels have a rectangular cross-section with a width $w$, a height $h$ and an aspect ratio of $\alpha=h / w$. The velocity profile can be calculated by integrating the Stokes equation. ${ }^{92}$ Figure 3-1 shows velocity profiles obtained from FEM flow field simulations of a rectangular microchannel. The channel has a width of $w=100 \mu \mathrm{m}$, whereas the height $h$ is varied from 20 to $100 \mu \mathrm{m}$. A parabolic Poiseuille flow profile is obtained along the narrow aspect of the channel ( $y$-axis), with the fluid in the center of the channel having the largest velocity. The averaged flow along the wider channel aspect ( $x$-axis) has a boundary-layer character. It varies rapidly near the walls and approaches a low gradient profile in the center of the channel. The distance over which the flow changes from the constant value to the stick boundary condition is approximately the narrow dimension. 


\section{Microfluidics}

Therefore, with decreasing $\alpha$ the average velocity in this direction is increasingly pluglike, whereas the velocity profile in the narrow direction remains parabolic.

\subsection{Viscoelastic materials in microflow}

Since the Stokes equation contains no time derivatives, all fluid-dynamical phenomena described by this equation are stationary. ${ }^{92}$ However, there are physical processes encountered in microfluidics, whose non-linearities may even increase with reducing device dimensions. ${ }^{91,98}$ Dissolved polymers introduce viscoelasticity to the fluid that further enriches the flow behavior by including purely elastic flow instabilities ${ }^{99}$ and elastic turbulence. ${ }^{100}$ Effects of viscoelasticity are most apparent under high rates of variation of stresses. The strength of non-linear effects depends on the Weissenberg number, ${ }^{101}$

$$
W i=\tau_{p} \dot{\varepsilon}
$$

where $\dot{\varepsilon}$ is the rate of deformation in the flow and $\tau_{\mathrm{P}}$ is the polymer relaxation time. When $W i$ is small, the polymer relaxes before the flow deforms it significantly, and perturbations from equilibrium are small. With $W i$ approaching unity, the polymer does not have time to relax and it is unraveled via a coil-stretch transition.

A second time scale characteristic of variations in the flow geometry may also exist. $^{90,91}$ In this case, the behavior of the complex material is characterized by a dimensionless Deborah number,

$$
D e=\frac{\tau}{t},
$$

with $\tau$ being a microscopic relaxation time characteristic of the molecular structure and $t$ being the time constant of the applied perturbation. De characterizes the intrinsic fluidity of a material.

When $D e$ is small compared to unity, liquid-type behavior is observed. At higher frequencies and therefore larger values of $D e$, the response is typical of an elastic

solid. ${ }^{90}$ Viscoelastic effects are fundamentally independent of $R e$ and should not diminish when the device is miniaturized. ${ }^{102}$ Performing experiments on a microscale eliminates inertial effects that compete or mask viscoelastic effects. Therefore, microfluidic devices have proved useful for both analyzing effects of fluid flow on polymer behavior and studying effects of polymers on the behavior of fluid flows. 


\subsection{Diffusion and Mixing}

Mixing is a basic process required for many biological, chemical, and physical applications. On the macroscopic level, the diffusion is described by Fick's first law, ${ }^{90}$

$$
j=-D \nabla c .
$$

It is used in the steady-state case and indicates that the diffusion flux $j$ is depending on the concentration gradient $\nabla c$. The coefficient $D$ is the diffusivity. If changes in concentration occur over time, they result in positional changes in diffusion flux. This is expressed by the continuity equation,

$$
\frac{\partial c}{\partial t}=-\nabla j
$$

Therefore, for non-steady or continually changing state diffusion, one has to use Fick's second law, ${ }^{90}$

$$
\frac{\partial c}{\partial t}=D \Delta c
$$

which is obtained by combining equation (3-8) and (3-9). In order to fully describe local concentration and concentration changes, the Navier-Stokes equation or - for purely laminar flow - the Stokes equation has to be additionally considered since it is coupled to equation (3-10). In general, the resulting system of coupled differential equations is very complicated and cannot be analytically solved. To describe the physics of the microflow inside the microchannel system, finite element simulations are therefore used (chapter 2.3).

\subsection{Hydrodynamic focusing}

Laminar flow conditions in microchannels force mixing to occur purely by diffusion. This can be desirable or not, depending on the application. However, unique flow phenomena on microscales can be leveraged to design and fabricate microfluidic devices according to experimental needs. For analyzing DNA compaction under highly defined conditions, hydrodynamic focusing devices are used. Hydrodynamic focusing features crossed (micro) channel geometry with three inlets and one outlet. Solutions injected in the microfluidic device meet at the confluence of the microchannels. The 

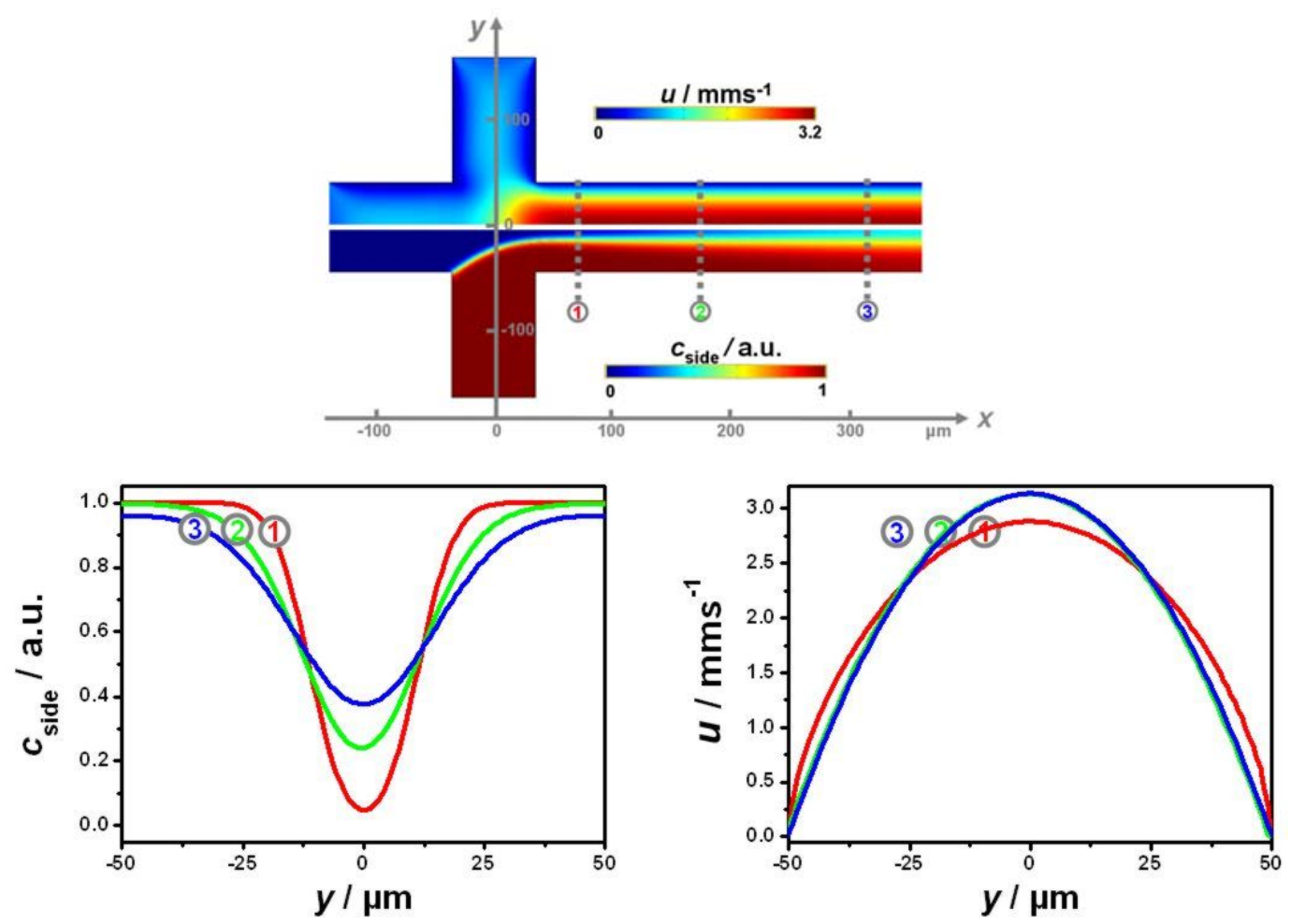

Figure 3-2: (a) Finite element simulations of the velocity field $u$ (top) and the concentration of side channel component $c_{\text {side }}$ (bottom) illustrating the effect of laminar mixing. (b) Line profiles of $u$ and $c_{\text {side }}$ at annotated positions along the main channel.

main stream is hydrodynamically focused by the influx of the solutions injected into the two side channels and fluid elements are accelerated. ${ }^{103}$ In addition to hydrodynamic focusing of a liquid stream, using two miscible liquids in crossed microchannels allows for diffusive mixing of these liquids. This opens a wide field of interesting experiments. ${ }^{56,60,104}$ The details of subsequent mixing between fluids vary greatly depending on the geometry of the outlet channel. In what follows, two fundamental mixing scenarios are discussed.

In Figure 3-2a, flow conditions in a hydrodynamic focusing device with an outlet channel of uniform width are calculated using 2D finite element simulations. Simulations describe the velocity field $u$ (top) and the concentration $c_{\text {side }}$ (bottom) of the side channel component evolving when aqueous solutions with identical viscosities $\eta_{\text {main }}=\eta_{\text {side }} \approx \eta_{\text {water }}$ are injected in the main and side channels with a fixed initial velocity $u_{0}=0.7 \mathrm{~mm}^{-1}$. Both side channel solutions contain small molecules of hydrodynamic radius $R_{\text {side }}=0.45 \mathrm{~nm}$ with a diffusion constant $D_{\text {side }}=5 \cdot 10^{-10} \mathrm{~m}^{2} \mathrm{~s}^{-1}$. 

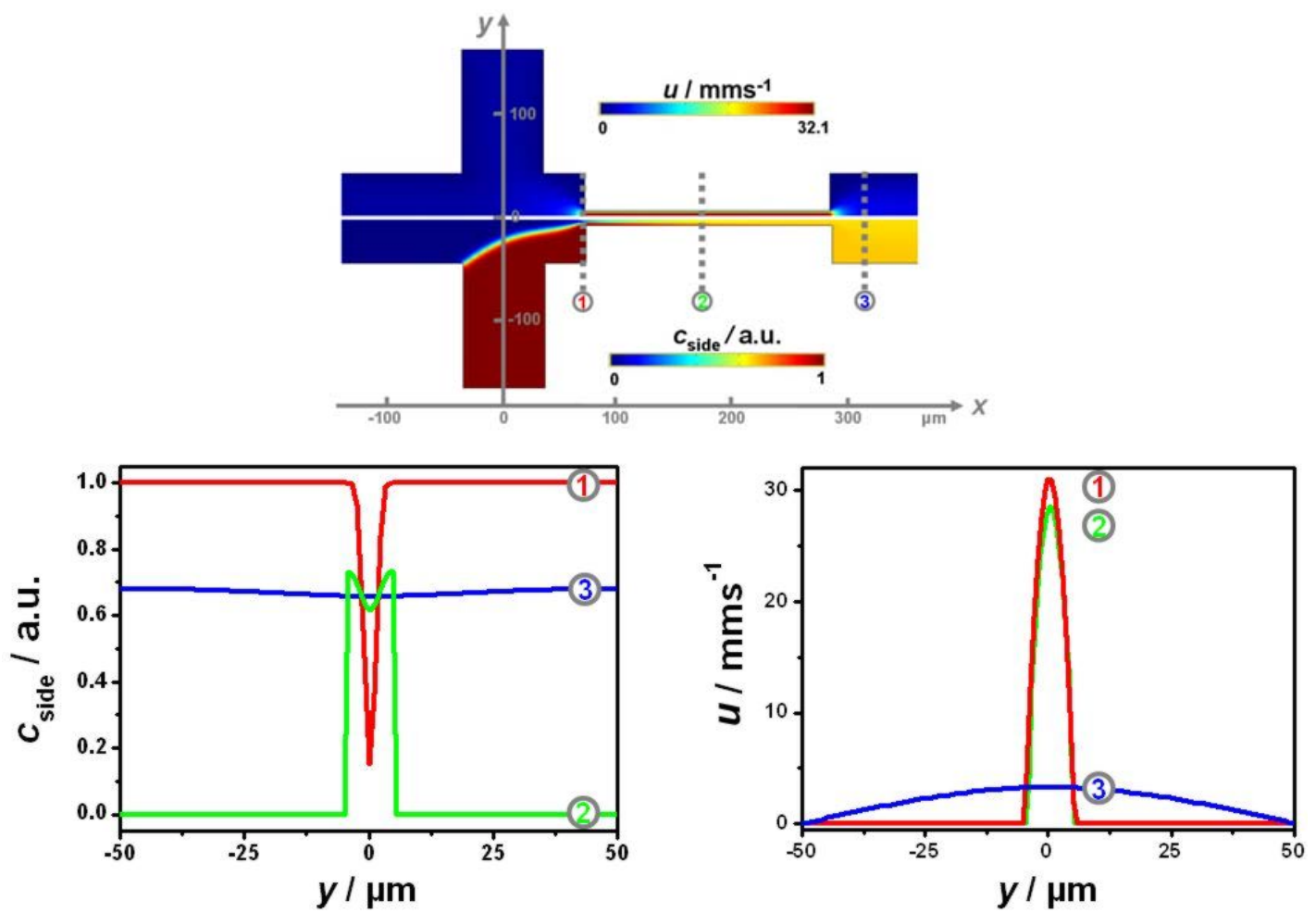

Figure 3-3: (a) Finite element simulations of the velocity field $u$ (top) and the concentration of side channel component $c_{\text {side }}$ (bottom) illustrating the effect of fast mixing in a device with a narrowed region in the outlet channel. (b) Line profiles of $u$ and $c_{\text {side }}$ at annotated positions along the main channel.

These values are comparable to those of small biologically relevant molecules such as glucose. $c_{\text {side }}$ ranges from 0 to 1.0 . Owing to the symmetry of the microfluidic device, it is sufficient to simulate half of the device.

Lines crossing the channels quantify values of $u$ and $c_{\text {side }}$ at different $x$ positions 1,2 , and 3 (Figure 3-2b). The mixing in the device shown in Figure $3-2 \mathrm{a}$ is a consequence of the laminar flow and the diffusion within the channel. A continuous increase in $c_{\text {side }}$ at the channel center $(y=0)$ is recorded. $c_{\text {side }}$ will approach a constant value at some further point $x$ when side and main components are completely mixed. Velocity profiles of this device approach a steady-state value at some distance from the confluence area. Taking advantage of the highly defined laminar flow conditions along the outlet channel, diffusive mixing establishes stable concentration gradients. Introducing distinct chemical reagents into the injected solutions, concentration gradients in devices having such geometry can be utilized to study non-equilibrium structure formation in the intermixed region. Each observation point contains a different concentration of reactants. Thus, a wide variety of chemical environments can be easily created. 


\section{Microfluidics}

Temporal resolution of particular time points in the reaction can be achieved by observing the state of the reaction at different positions along the outlet channel because distance in the flow direction translates into a time of reaction. ${ }^{103,105,106}$ Consequently, interaction dynamics depending on the concentration distribution can be spatially separated. Remarkably, non-equilibrium dynamics are thus accessible in steady-state flow. ${ }^{59-61}$

Besides establishing highly defined concentration gradients, rapid mixing of different solutions is often desired, e.g. to quantify time-evolved properties of a fully mixed ensemble. Figure 3-3a shows the geometry of a hydrodynamic focusing device with this ability. The inclusion of an abrupt decrease in the width of the interaction channel creates markedly different flow conditions than those in a straight channel having a uniform width. Finite element simulations of the second device have been performed using identical initial velocities and concentrations. However, the scale of $u$ is 10 -fold higher for the device in Figure 3-3a due to the rapid increase in $u$ in the narrow channel region. Line profiles at $x$ positions close to the beginning of the channel step (position 1) exhibit extremely steep $u$ and $c_{\text {side }}$ values moving from the center of the channel outward along the $y$-axis. The fast mixing induced by the strong reduction of the distance, which molecules have to cover by diffusion, results in a nearly constant value of $c_{\text {side }}$ at positions after the confined region (i.e. position 3 ).

The two scenarios only serve as examples. Interactions within microchannels can also be controlled by variables such as relative flow velocity and further geometric modifications. The width of the focused stream can be adjusted by the ratio of flow rates of the main channel and the side channels. Increasing the flow rate of the side channels leads to a narrowing of the center stream. Clearly, a wide variety of parameters are sufficiently malleable to enable investigations of real-time dynamic interactions. Furthermore, these parameters may be tailored to address specific experimental considerations.

An additional, important benefit of utilizing hydrodynamic focusing devices to study complex fluids results from the ability to introduce an additional hydrodynamic stress due to the sudden increase of the overall flow rate in the crossing region. Fluid elements become extended along the flow direction, as do the polymer molecules within the flow. Providing a sufficiently large rate of extension $\dot{\varepsilon}$ compared to $\tau_{\mathrm{P}}{ }^{-1}$ (high $W i$ ), polymer molecules are stretched and aligned during the self-assembly process. This improves the formation of liquid-crystalline phases, which allows for a significantly enhanced characterization by X-ray diffraction. ${ }^{59-61}$ Thus, additional information regarding the liquid-crystalline order of the complex fluid is obtained. However, biomaterials are typically sensitive to the high energy of X-rays and can rapidly degrade when exposed to a beam for long periods of time. Measurements in 
3. Microfluidics

microdevices eradicate this concern, because while the X-ray beam remains at a fixed position, the materials within the device continue to flow. As a consequence, each individual molecule is exposed to the X-ray beam only for a very short time, and the detector records an ensemble average instead of a time average. 


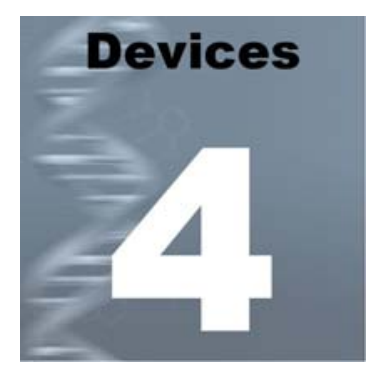

\section{Microfluidic DeVICES}

X-ray diffraction, ${ }^{105,} 107$ and, in particular, spatially resolved microdiffraction in hydrodynamic focusing microdevices ${ }^{59-62,108}$ provide new opportunities to study timeresolved reaction dynamics of complex fluids under controllable conditions. The characterization of these materials, which are typically liquid-crystalline under ambient conditions, is significantly improved owing to a concurrent orientation during selfassembly processes. ${ }^{59,61}$ However, a major obstacle for performing X-ray diffraction measurements directly on a microfluidic chip is the lack of cheap, tunable, and robust devices with thin, low-absorbing and low-scattering windows suitable for X-ray measurements. In this chapter, a straightforward and scalable method of fabricating inexpensive, long-lifetime X-ray microdiffraction compatible microfluidic devices is presented. To illustrate the analytic power and geometric flexibility of the microfluidic devices, X-ray microdiffraction measurements have been performed in order to study shear-induced alignment effects in the well characterized smectic liquid crystal system n-octyl-4-cyanobiphenyl (8CB). ${ }^{63}$ 

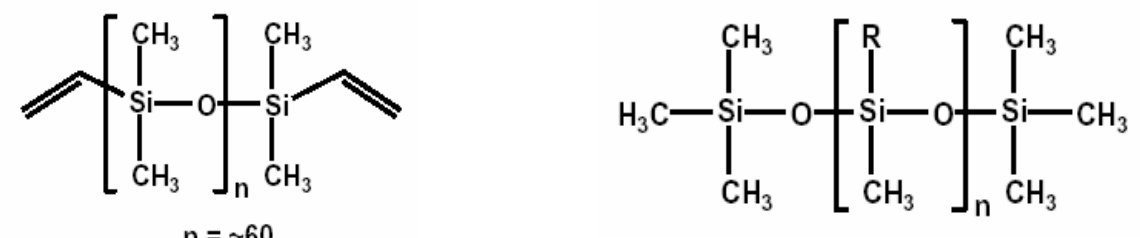

Figure 4-1: Chemical structure of PDMS: oligomer (left), crosslinked (right).

\subsection{Manufacturing microfluidic devices}

The utility of microfluidic devices to manipulate fluids is of widespread interest in many scientific and industrial contexts. Their design often requires unusual geometries and the interplay of multiple physical effects such as pressure gradients, surface tensions, and capillarity. The earliest microfluidic systems were fabricated in silicon by using technology originally developed for the microchip industry. ${ }^{109-111}$ For biological research, silicon is often not the ideal material for microfluidic applications due to optical opacity, difficulty in component integration, and unsuitable surface characteristics. Furthermore, faster and less expensive device fabrication is needed. In order to satisfy these demands, soft lithographical techniques to create microfluidic devices have been developed over the past two decades. ${ }^{112-114}$

\subsubsection{Soft lithography and standard PDMS devices}

Soft lithography involves the replication of a master structure into a soft elastomer, such as Sylgard 184 poly-(dimethylsiloxane) (PDMS, Dow Corning GmbH, Wiesbaden, Germany). Although several polymers (e.g. polyurethane) have been used for soft lithography, PDMS has a useful combination of properties. It is nontoxic, permeable for water and many gases, readily available commercially, and optically transparent down to a wavelength of approximately $\lambda \approx 300 \mathrm{~nm}$. It has a Young's modulus of about $1 \mathrm{MPa}$ (depending on cross-linking density) making it a moderately stiff elastomer. ${ }^{112}$ Consisting of repeating units of -O-Si $\left(\mathrm{CH}_{3}\right)_{2}$-groups (Figure 4-1), it is intrinsically hydrophobic (advancing contact angle of water: $\theta_{\mathrm{a}}\left(\mathrm{H}_{2} \mathrm{O}\right) \approx 110^{\circ}$ ). ${ }^{112,115}$ Typically, standard photolithography is used for manufacturing masters. Figure 4-2 shows the principle steps of master fabrication. A silicon wafer (Si-Mat, Landsberg/Lech, Germany) is cleaned with isopropanol and dried on a hotplate for 5 


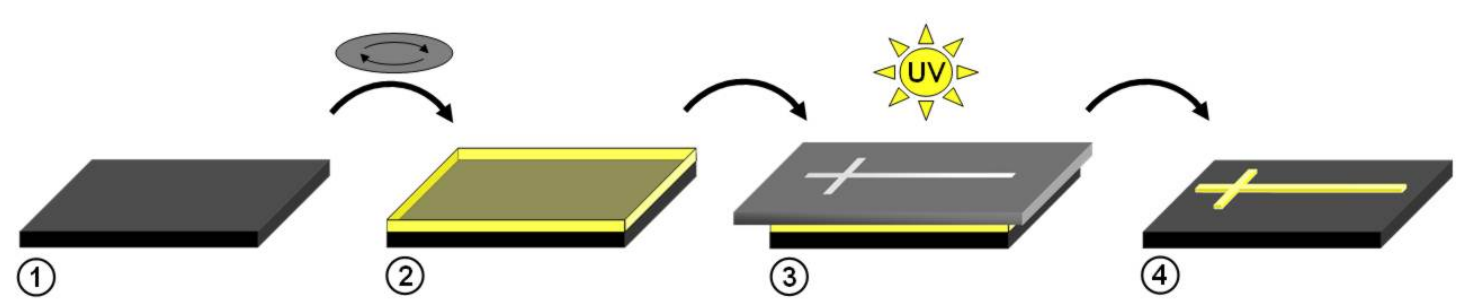

Figure 4-2: Schematic representation of principle steps of the master fabrication. (1) Clean silicon wafer as a substrate. (2) Spin coating the substrate with photo resist. (3) Exposure to UV light through a high resolution lithography mask. (4) Master containing the developed structure.

minutes at $200^{\circ} \mathrm{C}$. A layer of negative photo resist (SU-8, Micro Resist Technology $\mathrm{GmbH}$, Berlin, Germany) is spin coated onto the wafer. Depending on the type of photoresist and the spin coater (SCS, Indianapolis, USA) parameters, a thickness range of $50-200 \mu \mathrm{m}$ can be achieved. To polymerize selected regions of the photo resist, the wafer is exposed to UV light (wavelength $\lambda=365 \mathrm{~nm}$ ) through a lithography mask using a Karl Süss MJB3 mask aligner (Süss Microtech AG, Garching, Germany). Lithography masks are printed transparencies (JD-Photo-Tools Ltd., Oldham, UK) or chrome masks (ML\&C, Jena-Maue, Germany), with microstructures drawn using AutoCAD 2005 (Autodesk, München, Germany). For resist layers thicker than a few micrometers, an iterative exposure is necessary. In order to completely crosslink the exposed photo resist areas, the wafer is post-exposure baked and developed. After dissolving the unpolymerized photoresist, a positive relief of the channel structure is left on the wafer and cleaned with isopropanol. This structure acts as a master for casting PDMS channels.

To replicate the 3D microstructure in PDMS (Figure 4-3), the pre-polymer is mixed with the cross-linker (ratio 10:1 (w/w)), degassed thoroughly using an exsicator and poured onto the master. The liquid PDMS pre-polymer conforms to the shape of the master and replicates features of the master with high fidelity (10's of nm). ${ }^{116}$ After curing the pre-polymer at $65^{\circ} \mathrm{C}$ for approximately $3 \mathrm{~h}$, the PDMS replica is peeled off of the master. To prevent irreversible bonding between silicon and PDMS, the surface of the wafer is treated with heptafluoropropyl-trimethylsilane (97\%, Sigma-Aldrich, Germany) by absorption from the gas phase. Dimensional limits in channel design are in the range of 1 to 300 micrometers. The width of the channels is measured with optical bright field microscopy resulting in an accuracy of $\pm 0.2 \mu \mathrm{m}$.

To introduce fluids into the microchannels, Teflon tubing (NovoDirect, Kehl, Germany) with an inner diameter of $500 \mu \mathrm{m}$ and an outer diameter of $1000 \mu \mathrm{m}$ is used. 


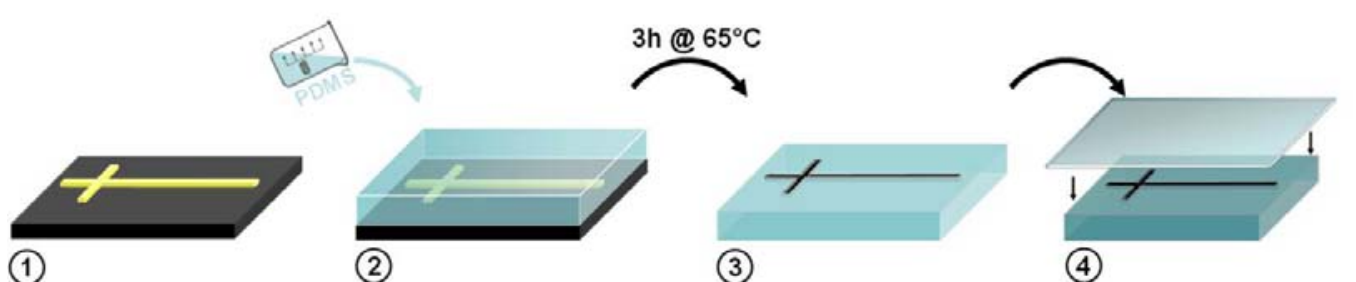

Figure 4-3: Schematic representation of the manufacturing process of a standard microfluidic device from an existing master. (1) Photolithographic fabrication of a master with relief structure. (2) Casting of PDMS against the master. (3) Peeled off PDMS replica after cross linking the PDMS at $65^{\circ} \mathrm{C}$. (4) Sealing the channel structure with a cover slide.

To connect the tubing, holes slightly smaller than the outer tube diameter are punched in the PDMS replica. When the tubing is inserted, it exerts pressure on the PDMS and provides a waterproof seal. To improve tightness, the tubes are additionally glued into the PDMS by using Loctite ${ }^{\circledR}$ 406/770 glue (Henkel Loctite, München, Germany).

The PDMS replica can be sealed irreversibly to PDMS, glass, silicon, polystyrene, polyethylene, or silicon nitride by exposing both the surface of PDMS and the surface of the substrate for 10s to an air plasma using a plasma cleaner (Harrick Scientific Corporation, Ossining, USA). Oxidization using plasma produces silanol ( $\mathrm{Si}-\mathrm{OH})$ groups on the PDMS surface, converting it to a hydrophilic form $\left(\theta_{\mathrm{a}}\left(\mathrm{H}_{2} \mathrm{O}\right) \approx 10^{\circ}\right),{ }^{112,115}$ and $-\mathrm{OH}$-containing functional groups on the other materials. These two types of polar groups form covalent -O-Si-O-bonds when the surfaces are brought into contact. ${ }^{111}$ For standard microfluidic PDMS devices, microchannels in the cross-linked PDMS stamp are sealed with a glass slide.

Elastomeric polymer molding enables the generation of topologically sophisticated microfluidic structures according to experimental demands. A significant advantage of this technique is the rapid turn-around time from conception of the experiment to fabrication. ${ }^{111}$ One master can be used several times to fabricate replicas, resulting in an extreme cost and time efficency. In addition, the list of advantages of microfluidic devices manufactured using PDMS includes robustness, reduced size of operating systems, flexibility in design, reduced use of reagents, increased speed of analyses, and easy integration with outside components because the polymer conforms to most materials.

When studying complex fluids in a microchannel device, the utilization of a multitude of methods of analysis is desirable. Asides from different optical techniques, X-ray microdiffraction in microflow in particular provides new opportunities to study complex fluids. ${ }^{59-62,108}$ Due to its optical properties, PDMS is compatible with many 


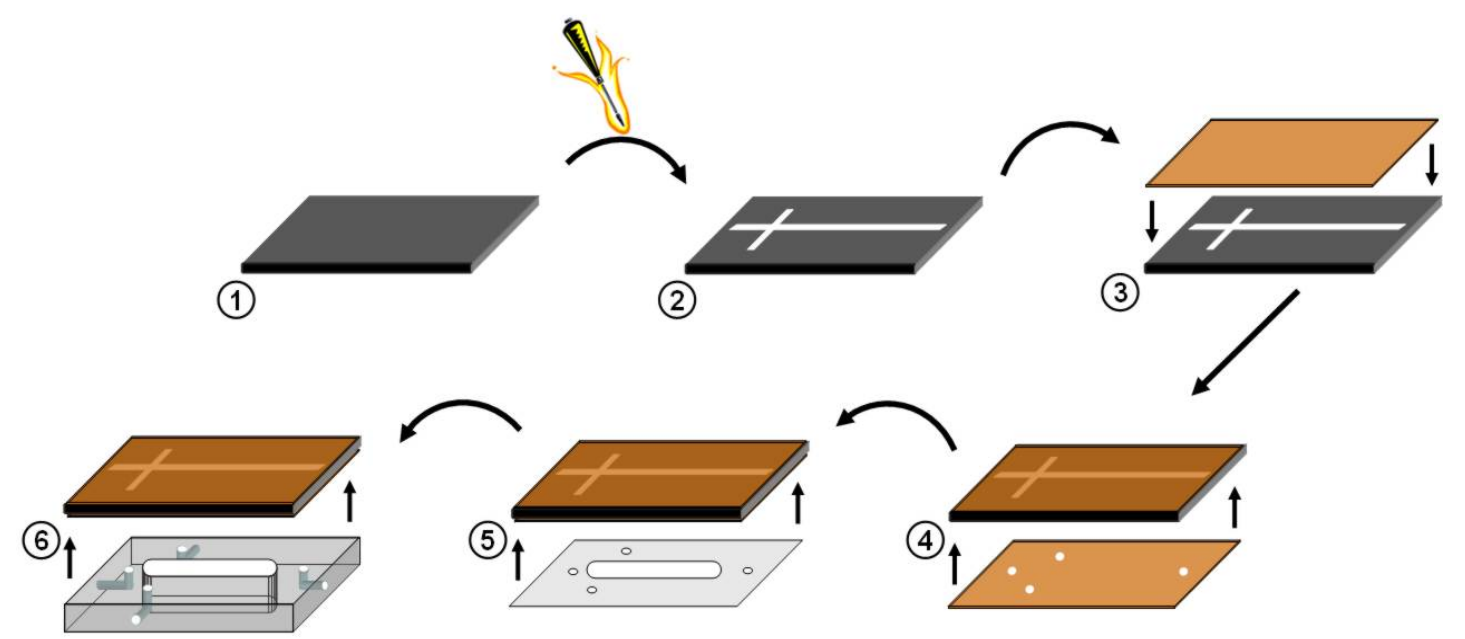

Figure 4-4: Schematic representation of the manufacturing process of KSK devices: (1) Stainless steel plate as starting point. (2) Spark erosion of the channel structure. (3) Covering with a thin self-adhesive Kapton foil on one side. (4) Closing the channel structure by covering the other side with a second Kapton foil. Four holes are punched into this Kapton foil, which fit the inlet positions of the microfluidic device. (5) Thin double side sticking tape with cavities at the inlet position and the measuring area is used for mounting the microfluidic device on a PMMA slab assisting the connections to the fluid pumping system (6).

optical detection methods. However, X-ray diffraction measurements directly on a microfluidic chip cannot be performed using standard PDMS devices. The major obstacle here for is the X-ray absorption and scattering properties of PDMS. In the following sections, two straightforward and scalable methods are presented for fabricating long lifetime X-ray microdiffraction compatible microfluidic devices with thin polyimide (Kapton) windows.

\subsubsection{Kapton-Steel-Kapton devices}

The production of the X-ray compatible Kapton-Steel-Kapton (KSK) microfluidic devices is illustrated in Figure 4-4. The microchannel structure is spark eroded in a thin stainless steel plate resulting in a microchannel structure, which is open on both sides. The thickness of the plate defines the height of the microchannels. Adhesive Kapton foils coated with a poly(siloxane) layer (thickness $53 \mu \mathrm{m}$, Dr.Müller GmbH, Allingen, Germany) are placed on both sides of the steel plate, such that the foils seal the device and serve as X-ray transparent windows to the microchannel. Four holes are punched into the bottom Kapton foil fitting the channel ends of the steel plate and serving as in- 


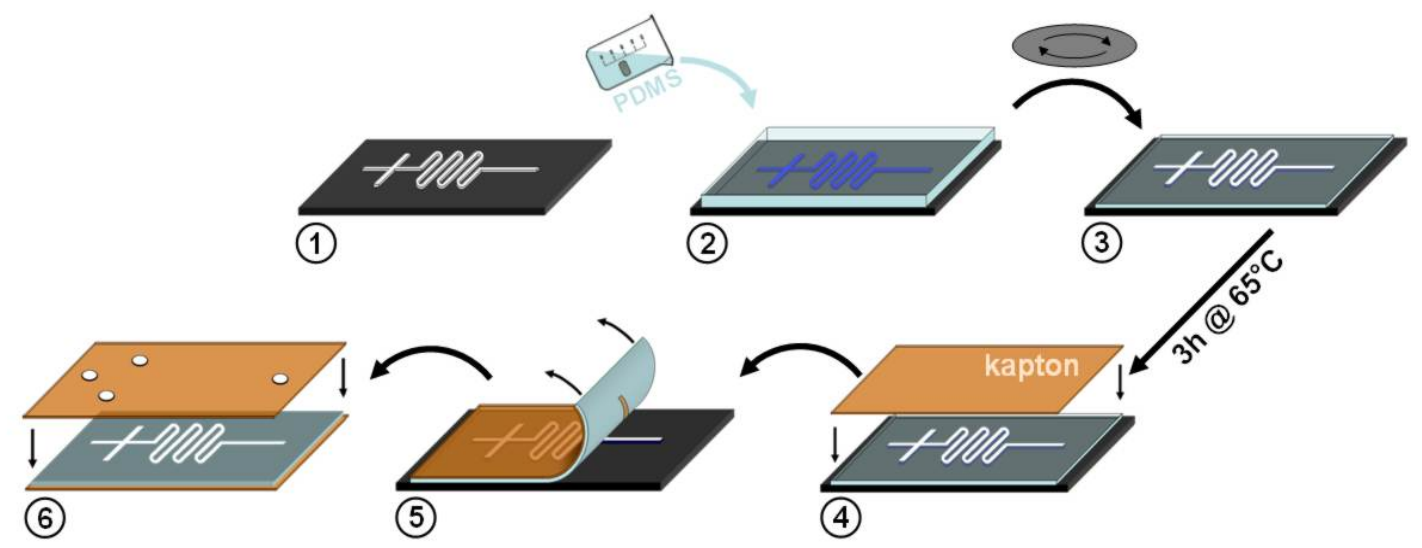

Figure 4-5: Schematic representation of the manufacturing process of microflow foils: (1) Photolithographic fabrication of a master with relief structure. (2) Casting of PDMS against the master (3) Adjusting of the PDMS layer to the height of the relief structure and cross-linking at $65^{\circ} \mathrm{C}$. (4) Covering with a thin self-adhesive Kapton foil. (5) Peeling off of the Kapton-PDMS replica. (6) Sealing of the channel structure with a second Kapton foil. Four holes are punched into the Kapton foil fitting the inlet positions of the microfluidic device.

lets of the microfluidic device. Using a thin double sided sticking tape with cavities at the inlet positions and the measuring area, the microfluidic device is mounted on a poly(methylmethacrylate) (PMMA) slab assisting the connection to the fluid pumping system. The center region of the PMMA support is milled out to provide an undisturbed pathway for X-ray beams. The connection to the pumping system is established by Teflon tubing implemented into male nuts (ProLiquid, Überlingen, Germany) that are screwed in the sockets of the PMMA support. Channels of the KSK devices have a width of 100-150 $\mu \mathrm{m}$ and a depth ranging from $200-300 \mu \mathrm{m}$.

Kapton has been acknowledged as a useful material for X-ray windows as it is both resistant to high flux densities of microfocused X-ray beams and shows low X-ray absorption in the keV energy range. Therefore, using ultra-thin Kapton foils enables the performance of high quality X-ray microdiffraction experiments. The biggest advantages of KSK devices are their robustness and the fact that the Kapton foils can be discarded, and then the microchannel structure in the stainless steel plate can be cleaned and reused with new Kapton foils. However, technical limitations of the spark eroding process restrain the abilities of KSK devices: the minimum channel width is approximately $50 \mu \mathrm{m}$, and smaller structures are not feasible. In addition, only simple geometric channel designs are realizable. Since the total length of the device is limited 


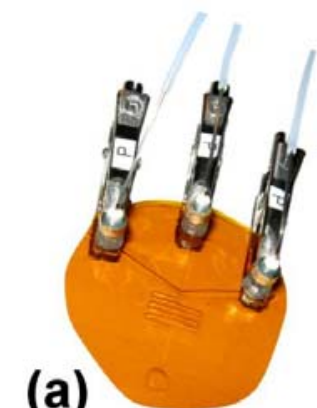

(a)

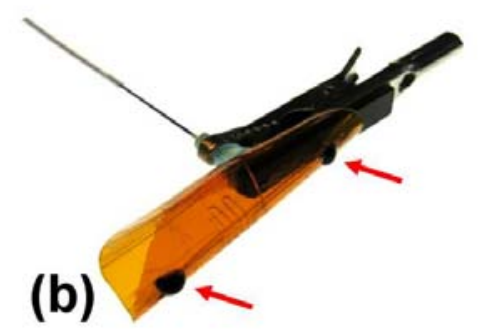

Figure 4-6: Photographs of connected microflow foils and the clipping system.

$(<3 \mathrm{~cm})$, the total observable reaction time is quite restricted making KSK devices inappropriate for the observation of many reactions.

\subsubsection{Microflow foils}

Microflow foils are the second type of X-ray compatible microfluidic devices used in this work. Based on the principle ideas of soft lithography, microflow foils outperform previously reported microchannel setups ${ }^{60,61,105,107,108}$ due to their inexpensive and rapid manufacturing steps as well as their overwhelming versatility with respect to channel design elements. The fabrication of these devices is shown in Figure 4-5.

The first steps of fabricating a master are the same as the standard soft lithography techniques described in chapter 4.1.1. In a next step, PDMS is spin-coated atop the master and excess PDMS is gently scraped off manually. The PDMS elastomer is subsequently cross-linked for three hours at $65^{\circ} \mathrm{C}$. A Kapton adhesive foil is used to seal the device, which assists peeling the PDMS off the master. The channel structure is sealed from the other side with a second Kapton foil. Four holes are punched into this Kapton foil fitting inlet positions of the microfluidic device. Press fitting clipping systems consisting of a modified crocodile clip (Conrad Electronics, Hirschau, Germany) and a syringe needle (Braun Sterican, 500 $\mu \mathrm{m}$ in diameter, VWR, Darmstadt, Germany) are used for connecting the sandwich structured microflow foils to the pumping system via Teflon tubes.

The Kapton-PDMS-Kapton microflow foils are a sophisticated addition to the field of microfluidic microdiffraction. In addition to the advantages of conventional soft lithographic devices described in chapter 4.1.1, an unprecedented versatility is achieved by using mechanically flexible tapes. The developed microflow foils facilitate the production of robust devices with ultra-thin walls that are highly adaptable in shape to 


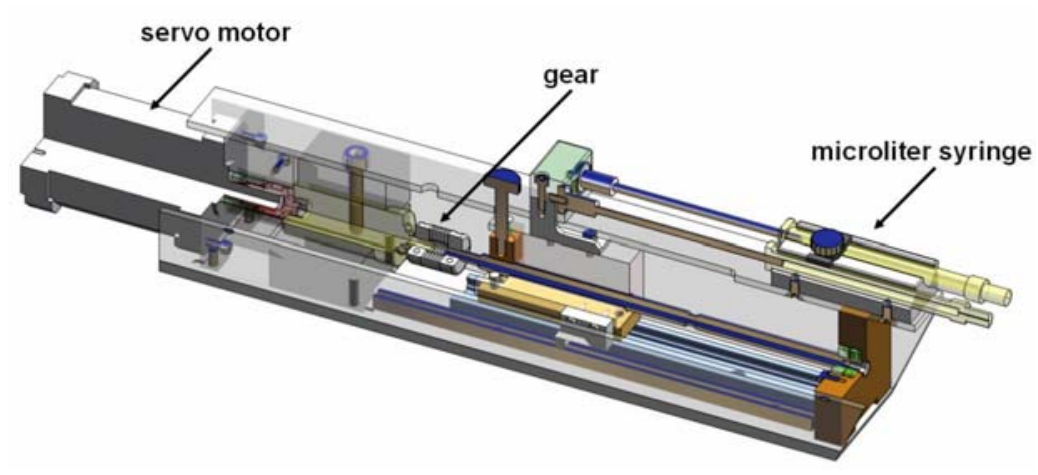

Figure 4-7: Drawing of the custom made syringe pump.

experimental requirements. To demonstrate this, colored water has been injected through the microchannels of a curled microflow foil resulting in droplets at the channel outlets (marked with arrows, Figure 4-6). The adhesion between the layers of microflow foils is strong enough to successfully pump fluids having viscosities on the order of $10^{4} \mathrm{mPa}$ s and higher.

This new technology is not limited to X-ray measurements, since all kind of different tapes can be used instead of Kapton foils - as long as these tightly bond with the intermediate PDMS layer. For instance, metal foils or optically transparent foils offer the ability to conduct reflectivity or optical microscopy investigations on complex fluids in microflow. Furthermore, the use of tapes also allows for facile incorporation of non-sealing layers such as filter papers and membranes.

\subsubsection{Controlling the microflow}

To control and manipulate the flow inside the microchannels, a home built syringe pumping system is used. Servo EC motors (Systro GmbH, Eiterfeld, Germany) with a very high accuracy of 10000 impulses per revolution are used to control the feed of up to two Hamilton microsyringes (10-500 $\mu$; Zefa, Harthausen, Germany) connected to the microchannels. To power the motors, a LabVIEW computer interface (National Instruments Corporation, Austin, TX, USA) is used.

At a gear transmission ratio of 546.75:1 inside the servo pumps, the pump setup allows for a continuous pumping in the range of $\mathrm{fL}^{-1}$ and $\mu \mathrm{L} \cdot \mathrm{s}^{-1}$ using fluids with a viscosity up to $10^{4} \mathrm{mPa}$. This results in experimental flow velocities between $35 \mathrm{~nm}^{-1}$ and $12.2 \mathrm{~mm} \mathrm{~s}^{-1}$ for a microchannel device with a square channel profile of $150 \mu \mathrm{m}$ in width and $500 \mu 1$ syringes. By varying the gear transmission ratio, the dynamic range of the pumps can be extended another three orders of magnitude. 


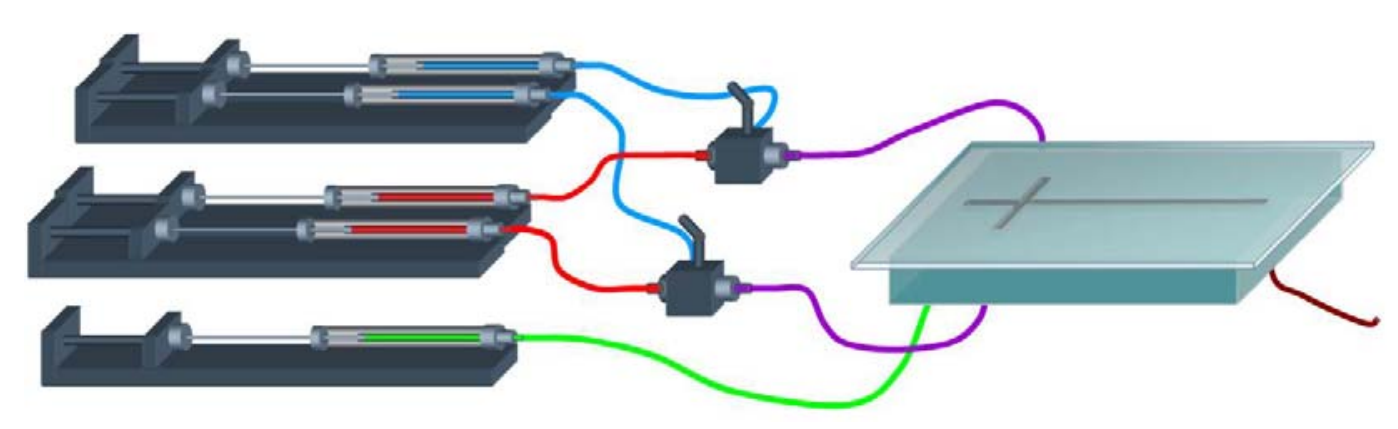

Figure 4-8: Microfluidic setup utilizing five microliter syringes.

Typically, five microliter syringes are utilized in total, one for the main channel and two for each of the two side channels. The connection of the two syringes feeding the side channels is established via a switch-able T-junction in a manner such that the content of both syringes is continuously added to the side streams. By varying the coflow in the side channels, the concentration of reactants during the measurement can be adjusted.

\subsection{Probing microflow foils}

X-ray diffraction ${ }^{105,107,117}$, and in particular spatially resolved microdiffraction in hydrodynamic focusing microdevices ${ }^{59-61,108}$ enables the analysis of time-resolved reaction dynamics of complex fluids under controllable conditions. X-ray compatible microflow foils introduced in chapter 4.1.3 provide new opportunities to study complex fluids. In order to illustrate the analytic power and geometric flexibility of these newly developed microflow foils, X-ray microdiffraction measurements have been performed in order to study shear-induced alignment effects in the well characterized smectic liquid crystal system n-octyl-4-cyanobiphenyl (8CB). ${ }^{118,119}$

\subsubsection{The liquid crystal system $8 C B$}

Smectic liquid crystals consist of elongated molecules that are aligned and arranged in layers. The chemical structure of the liquid crystal $n$-octyl-4-cyanobiphenyl (8CB, Sigma-Aldrich, Germany) is shown in Figure 4-9. 8CB is a thermotropic liquid crystal that exhibits a transition from nematic to smectic A at a temperature $T=33.5^{\circ} \mathrm{C}$. At 


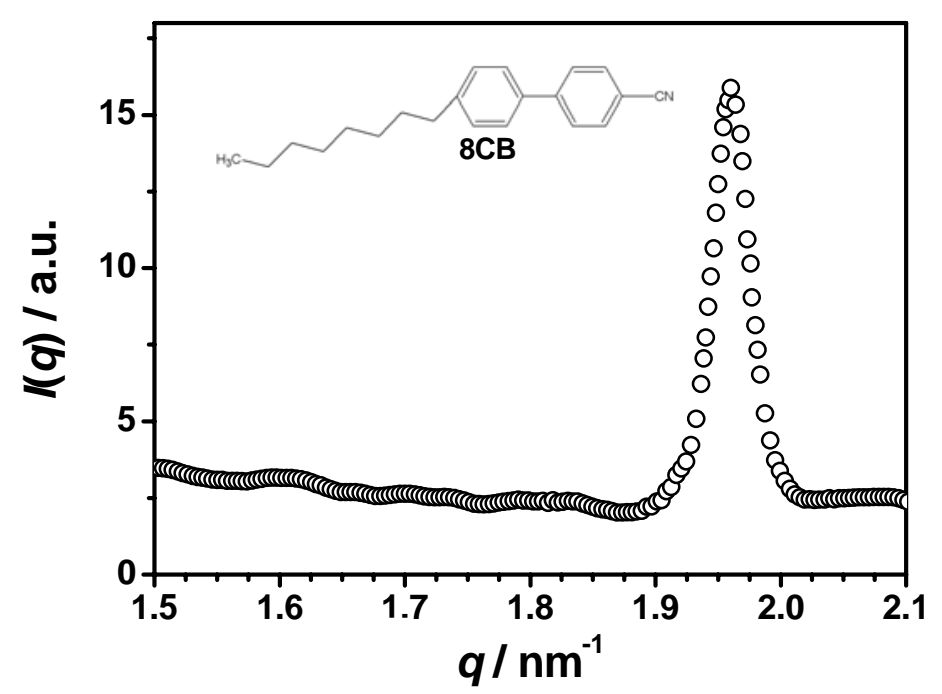

Figure 4-9: Scattering intensity plotted versus momentum transfer $q$ showing the peak of the smectic $A$ layer spacing at a position $q_{0}=1.98 \mathrm{~nm}^{-1}$. The chemical structure of the liquid crystal 8CB (4'-n-octyl-4cyanobiphenyl) is given as an inset.

room temperature, $8 \mathrm{CB}$ appears as a viscous fluid and forms layers displaying a smectic A liquid-crystalline phase. ${ }^{118,119}$ The smectic A layers of $8 \mathrm{CB}$ have a spacing of $d=3.17 \mathrm{~nm}$, which is easily accessible by the in-house as well as the synchrotron Xray microdiffraction setup. This is readily seen in Figure 4-9, which contains a plot of scattering intensity versus momentum transfer $q$ showing a prominent peak at a position $q_{0}=2 \pi / d, q_{0}=1.98 \mathrm{~nm}^{-1}$. The mesoscale assembly of $8 \mathrm{CB}$ molecules is strongly influenced by external stress. ${ }^{120,121}$ External stress can be applied and its influence analyzed by geometrically constraining the complex fluid inside a microfluidic device. ${ }^{103}$

\subsubsection{Characterization of the microchannels}

The channel structure of the microflow foils used for the $8 \mathrm{CB}$ experiments consists of a crossing area where three incoming channels meet. The height of the channels is $150 \mu \mathrm{m}$ and the width varies from $50 \mu \mathrm{m}$ to $150 \mu \mathrm{m}$. The device was constructed for enabling flow focusing, however in the experiments described below, the liquid crystal $8 \mathrm{CB}$ is injected in all three inlet channels. Flow velocities range between $10 \mu \mathrm{m}^{-1}$ and $500 \mu \mathrm{m} \mathrm{s}^{-1}$. The intersection area of the microflow foil is followed by a meandering channel section consisting of successive U-shaped turns. Due to the winding channel 


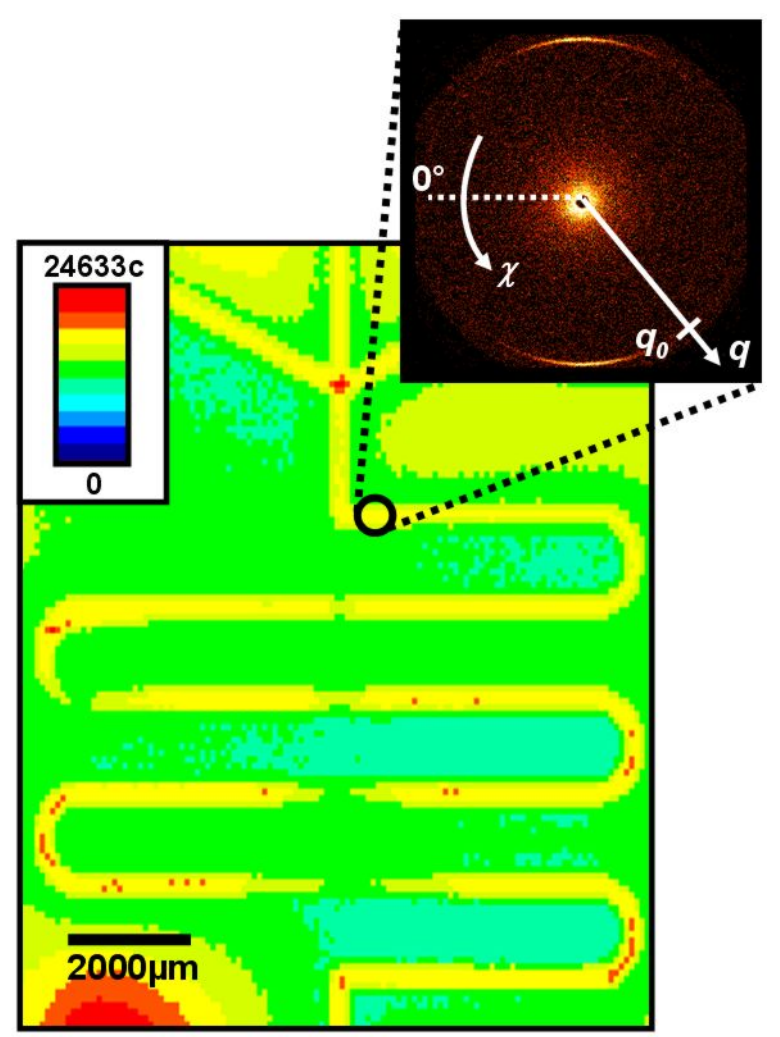

Figure 4-10: Scanning X-ray absorption image (step size $0.1 \mu \mathrm{m}$, exposure time $0.5 \mathrm{~s}$ ) of the microfluidic channel system using the in-house setup with a beam size of $d_{N} \approx 200 \mu \mathrm{m}$. The inset picture shows the 2D liquid-crystalline diffraction pattern of $8 \mathrm{CB}$. The reciprocal vector $q$ and the azimuthal angle $\chi$ are noted.

course, it is possible to extend the length of the channel significantly without increasing total lateral dimensions of the flow cell. Four hyperbolically shaped channel contractions down to $50 \mu \mathrm{m}$ in width are positioned horizontally along the channels.

X-ray absorption scanning images (Figure 4-10) of the entire device can be obtained with the in-house diffraction setup by lateral and vertical scanning with a minimum step-size of $50 \mu \mathrm{m}$, allowing for precise selection of measurement points. The channel structure is observable due to the lower absorption of $8 \mathrm{CB}$ compared to PDMS. The typical 2D scattering pattern of $8 \mathrm{CB}$ flowing through microchannels in the inset picture shows an orientation, which corresponds to an alignment of flowing smectic $8 \mathrm{CB}$ layers in the flow direction parallel to the channel course. The rod-shaped $8 \mathrm{CB}$ molecules are oriented perpendicular to the smectic A layers (Figure 4-12 right). For further details on the experimental X-ray setup see chapter 2.2 . 


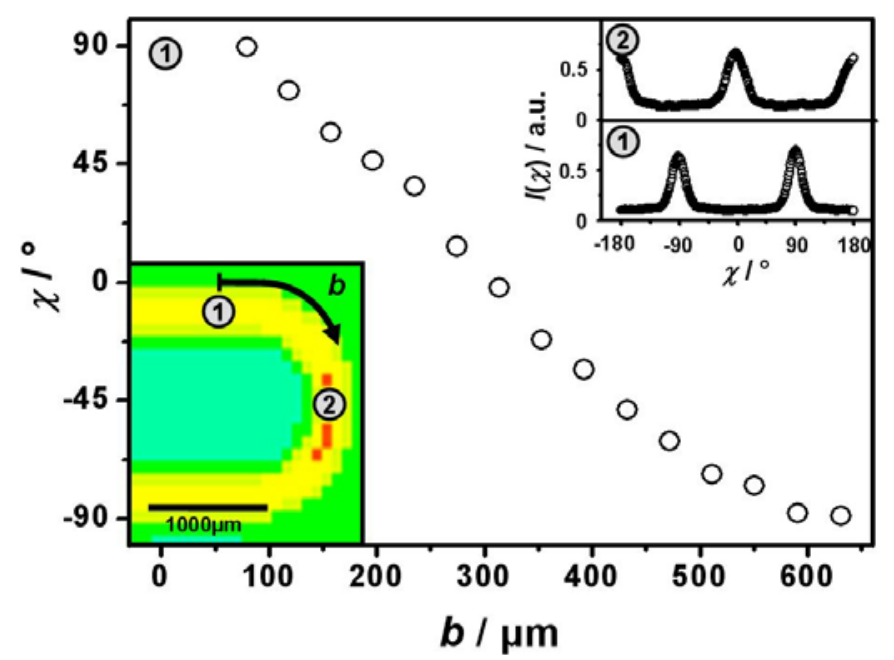

Figure 4-11: Orientation $\chi$ of the azimuthal intensity distribution of smectic A layers of the liquid-crystalline phase along the contour $b$ of a U-turn represented in the scanning $X$-ray absorption image. In the top section, corresponding azimuthal intensity distributions along the ring of the $q_{0}$ peak for two selected positions are shown to illustrate the effect of the U-turned channel shape.

\subsubsection{Geometrically induced bending of $8 C B$ smectic $A$ layers}

In Figure 4-11, the dependence of the orientation of 8CB smectic A layers on the local course of the microchannel is displayed along the contour $b$ of an U-shaped turn. The orientation of $8 \mathrm{CB}$ layers is induced by the geometry of the curve. A continuous change in orientation of $180^{\circ}$ of the complex fluid is observed, suggesting the suitability of this geometry for tracking bending processes.

Complex fluids undergo significant changes in their structural behavior when they are confined on the microscale under applied shear. ${ }^{122}$ Alignment of liquid-crystalline phases can be achieved by injecting liquid crystals into microchannels. ${ }^{121}$ However, this generally results in an imperfect ordering of molecules or a coarsening into intrinsically singly oriented microdomains. ${ }^{123,124}$ In Figure 4-12, the full width at half maximum $\Delta \chi$ of the azimuthal intensity distribution as a function of the position along the contour length $b$ is shown. In the horizontal parts of the channel, a width of $\Delta \chi=(15.6 \pm 0.6)^{\circ}$ is observed. However, by gradually changing the channel course in the U-turn region, local shears pointing in slightly different directions act on the confined complex fluid. This results in an orientational coarsening into oriented microdomains, each having discrete liquid-crystalline alignments. Due to the diameter $d_{\mathrm{N}} \approx 200 \mu \mathrm{m}$ of the in-house 

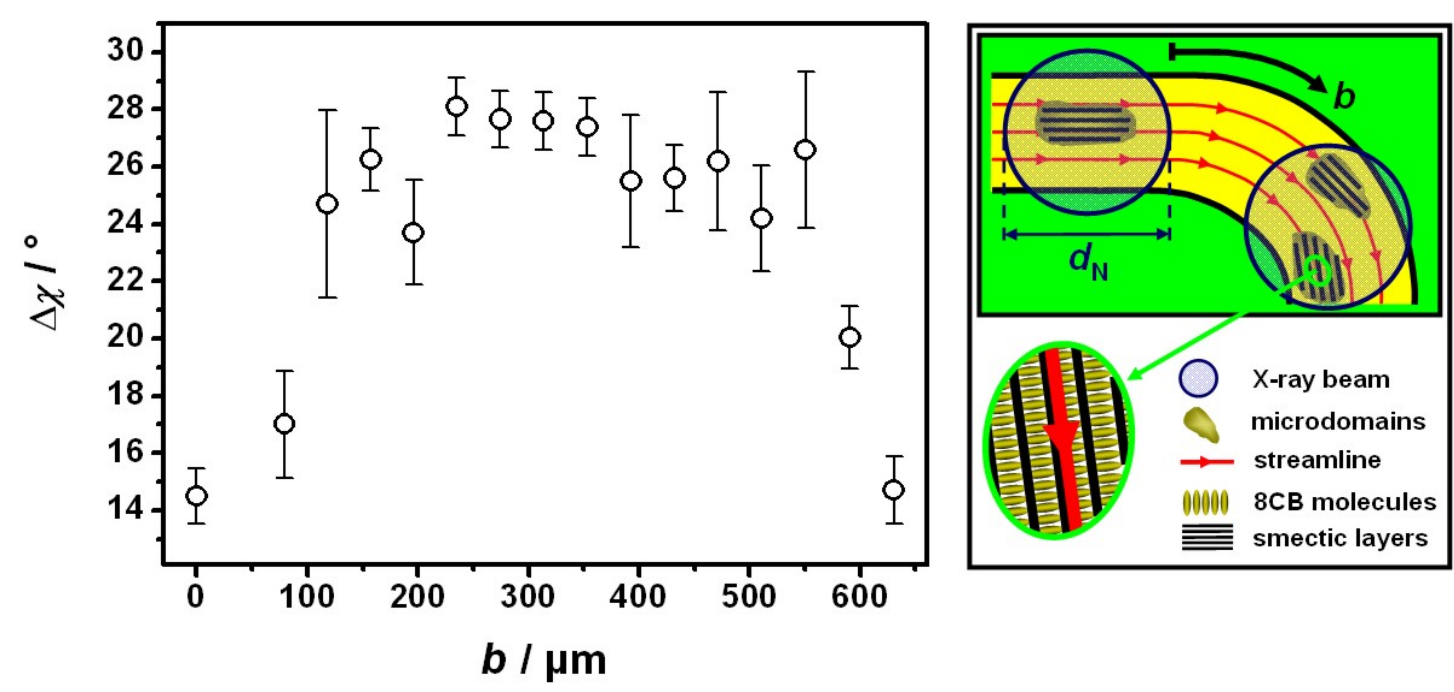

Figure 4-12: Width $\Delta \chi$ of the azimuthal intensity distribution of the smectic A layers of the liquid-crystalline phase along the contour $b$ of a U-turn. The effect of the beam size $d_{\mathrm{N}}$ and the discretely oriented microdomains are schematically demonstrated.

X-ray beam, different microdomains contribute to the measured signal (Figure 4-12), leading to the observed increase in width $\left(\Delta \chi=(27.6 \pm 0.6)^{\circ}\right)$. In addition, bending of the smectic A layers within microdomains may contribute to $\Delta \chi$.

\subsection{4. $8 C B$ in the hyperbolic channel sections}

When a fluid element passes through one of the hyperbolic contractions, it becomes extended along the flow direction as does the complex fluid within it. Additionally, a geometrically induced acceleration of the fluid elements is generated leading to an improved orientation of the confined complex fluid. Using finite element simulations, the velocity field and the strain rate in the hyperbolic channel regions is determined. As an example, Figure 4-13 shows a simulation of the velocity field in the hyperbolic region with a length of $2 \mathrm{~mm}$ along with arrows conveying the magnitude of velocity per position. The channel geometry has a specific advantage in terms of the fluid flow within the device: the velocity increases and decreases along the channel in a linear fashion. This is seen in Figure 4-13b, where the velocity is plotted against position $x$ along the middle of the channel. The mechanical implications of this linear velocity increase stem from the fact that the strain rate is described as the gradient of the velocity. It follows that a constant strain rate exists in the hyperbolic channel, with a change in sign occurring at the midpoint of the channel (Figure 4-13c). The length of 

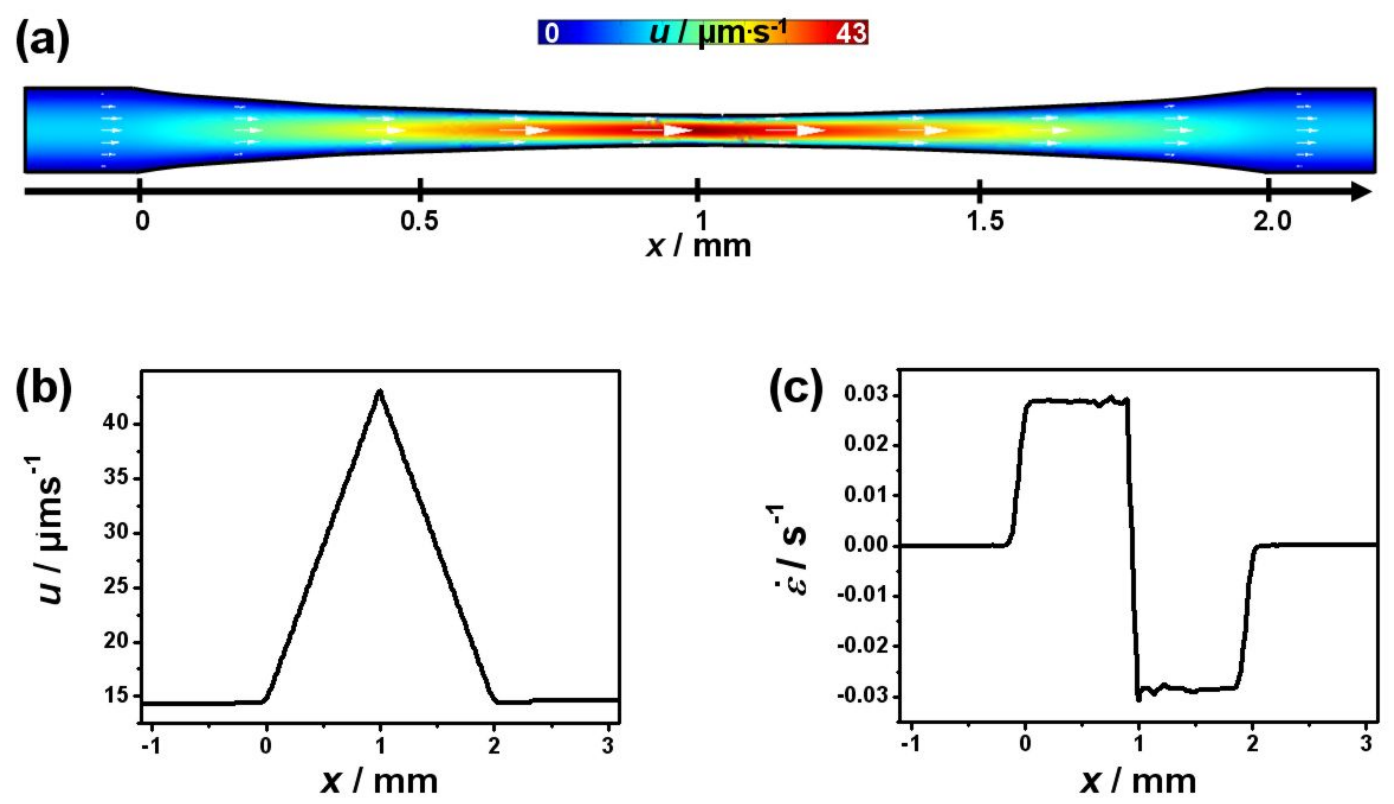

Figure 4-13: The velocity profile in a hyperbolic region (a) is linear in position $x$ (b) and results in a constant strain rate $\dot{\varepsilon}$ (c). The orientation parameter $\Delta \chi$ of $8 \mathrm{CB}$ diffraction peaks within the channel has a minimum value at $x=1 \mu \mathrm{m}$, the middle of the hyperbolic region.

the hyperbolic channel sections is varied in a manner such that, due to the linear mean velocity profiles, the mean shear rate is successively doubled in each hyperbolic area. Mean shear rates used for the measurements are in the range of $0.05 \mathrm{~s}^{-1}$ to $4 \mathrm{~s}^{-1}$ for the shortest and the longest hyperbolic section, respectively.

Figure 4-14a shows the width $\Delta \chi$ of the azimuthal intensity distribution as a function of the position $x$ for the four hyperbolic contraction regions measured using the in-house setup at a flow velocity of $10 \mu \mathrm{m}^{-1}$. Corresponding 2D scanning absorption images of the hyperbolic channel areas are shown for clarification. In regions with uniform channel geometry, a width $\Delta \chi=(15.6 \pm 0.6)^{\circ}$ is observed. The increased shear in the hyperbolically shaped channel regions forces microdomains to orient along the course of the stream. Since multiple microdomains are contributing to the measured signal, a decrease of orientational ordering is observed. This corresponds to an increase in $\Delta \chi$ ranging from approximately $2.1^{\circ}$ (Figure 4-14a(IV)) to $7.2^{\circ}$ (Figure 4-14a(I)) when approaching the ends of the contraction sections (inset of Figure 4-14a).

An increase in shear induced orientation is expected when moving from the ends to the middle of the hyperbolic contractions. The in-house beam size of $d_{\mathrm{N}} \approx 200 \mu \mathrm{m}$ being a limiting factor, it is not possible to resolve this improvement in orientation for the first hyperbolic channel section (Figure 4-14a(I)) having a length of $500 \mu \mathrm{m}$. However, with increasing length of the hyperbolic acceleration region, the limiting effect of the finite 


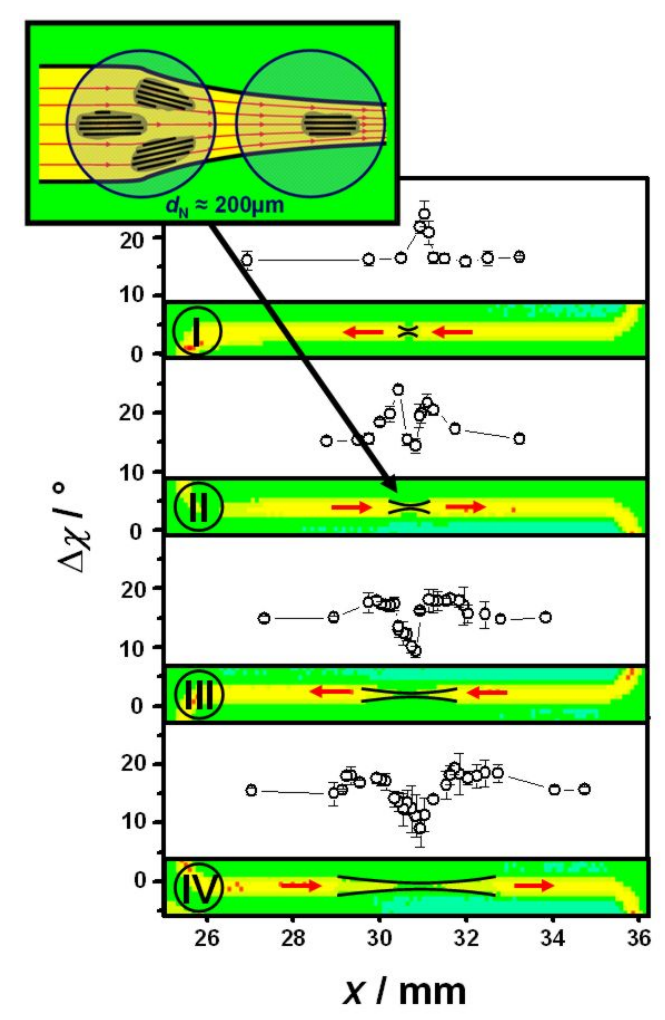

(a)

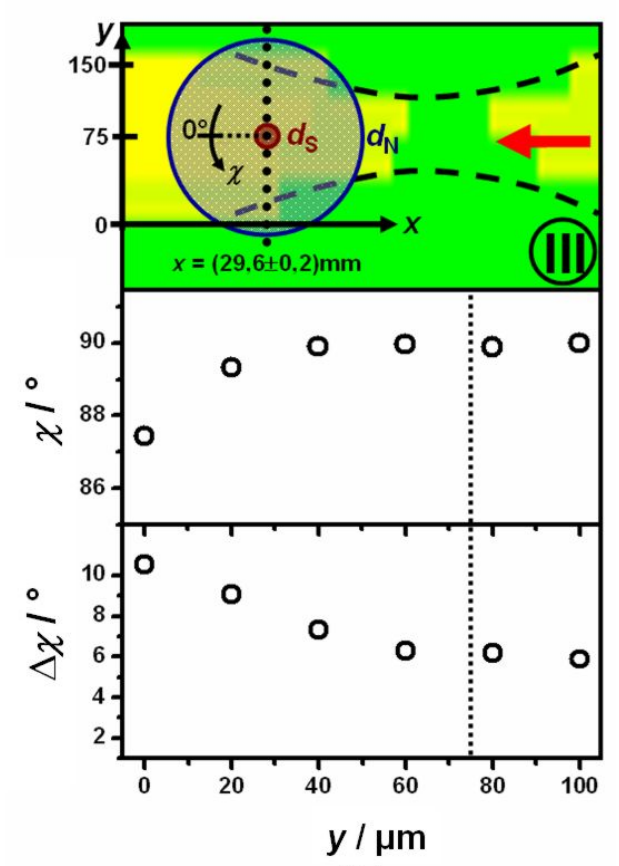

(b)

Figure 4-14: (a) Width $\Delta \chi$ of the azimuthal intensity distribution as a function of the $x$-position for all hyperbolic channel profiles measured using the inhouse setup. Effects of the beam size $d_{\mathrm{N}}$ and the discretely oriented microdomains, whose existence is implied by the scattering, are schematically demonstrated (inset). (b) Orientation $\chi$ and width $\Delta \chi$ of the azimuthal intensity distribution along the $y$-axis, measured using the microfocused synchrotron $X$-ray setup. The dotted line indicates the center of the channel. The top section shows the corresponding channel domain with synchrotron $\left(d_{S}\right)$ and in-house $\left(d_{\mathrm{N}}\right)$ beam diameter.

beam size is reduced. Therefore, the expected shear-induced increase in orientation is observed, as evidenced by the decrease in width to $\Delta \chi=(9.1 \pm 1.5)^{\circ}$ (Figure 4-14a(IIIIV)).

Using the synchrotron microdiffraction setup, ${ }^{78}$ the spatial resolution is improved by an order of magnitude due to the microfocused beam. The different beam sizes of the inhouse and synchrotron setup are illustrated in the top section. Figure 4-14b shows measurements perpendicular to the channel at a position close to the end of a hyperbolic section. Applying a flow velocity of $500 \mu \mathrm{m} \mathrm{s}^{-1}$ (mean strain rate $0.5 \mathrm{~s}^{-1}$ ), a remarkable decrease in width of the azimuthal intensity distribution down to $\Delta \chi=(5.9 \pm 0.6)^{\circ}$ is observed when approaching the maximum flow velocity in the center of the channel. 


\subsection{Conclusions}

In summary, the rapid prototyping of newly developed X-ray compatible microfluidic devices has been demonstrated. Straightforward soft lithographic fabrication techniques result in microchannel devices that are inexpensive, flexible, resistant to intense microfocused X-ray beams, and exceptionally useful for prototyping tailored microfluidic devices with sophisticated microfluidic geometry. The development of these microfluidic devices now places researchers in an advantageous position to approach important biophysical questions regarding the self-assembly of biomatter and interactions in a variety of environments. It is anticipated that especially microflow foils will provide a robust and affordable approach to continuous flow X-ray microdiffraction that will have broad applicability in material sciences, e.g. in micro total analysis systems (" $\mu$-TAS") and bioassays. The experiments on $8 \mathrm{CB}$ lay a foundation for future experiments involving biomaterials. The same principles demonstrated with the $8 \mathrm{CB}$ system (i.e. alignment in shear fields and confining geometries as well as induced rotation) can be measured with liquid-crystalline biological matter. In particular for accurate studies on the behavior and compaction of DNA in flow, the microfluidic devices are an indispensable starting point. 
5. DNA in Microflow

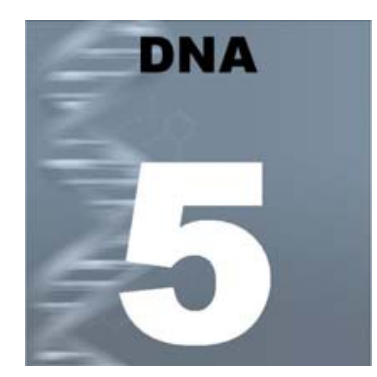

\section{DNA IN MICROFLOW}

DNA plays an exquisite role among the constituents of living systems. Therefore, an understanding of its phase behavior and knowledge of its structural features at each phase are of fundamental importance. In this chapter, liquid-crystalline properties of semi-dilute DNA solutions are discussed. Since it is highly desirable to study DNA solutions on a molecular length scales, X-ray diffraction experiments are used to follow the DNA inter-helix spacing between nearest neighbors, when increasing progressively the polymer concentration. Besides bulk properties, it is of crucial interest for this study, to analyze and characterize the behavior of semi-dilute DNA solutions in microflow. A detailed study of this behavior provides a first step towards understanding of the more complex process of DNA condensation in microchannels. Performing experiments under continuous flow conditions in hydrodynamic focusing devices, the influence of external stress on biomacromolecules can be analyzed and the molecules can be oriented. In addition, confocal Raman microscopy enables direct sample imaging at high resolution in specific consideration of the local molecular structure without using any markers. 

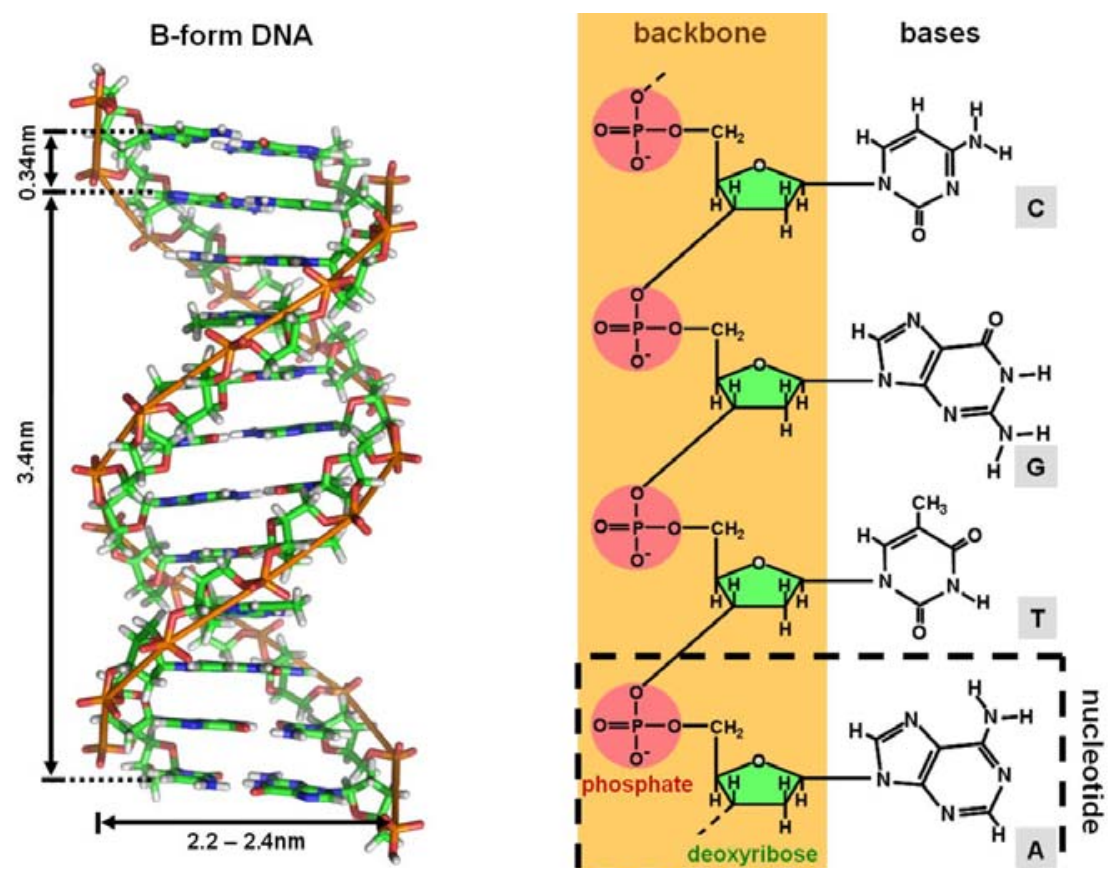

Figure 5-1: Structure and dimensions of B-form DNA. ${ }^{125}$

\subsection{General characteristics of DNA}

The most important and most abundant form in which DNA can be found in nature is the double-helical B-form. ${ }^{126}$ In this conformation, the highly negatively charged, water-soluble double helices have a diameter of about $2.0 \mathrm{~nm}^{127}$ and can reach gigantic lengths up to several meters. The persistence length of the semi-flexible polymer chains is in the range of $30-100 \mathrm{~nm} .{ }^{128}$ In eukaryotic cells, the huge DNA molecules are situated in nuclei with diameters of $5-10 \mu \mathrm{m} .{ }^{129}$ These four numbers ranging from the nano- to the macroscale elucidate the multi-dimensional character of DNA, which gives rise to extremely interesting and unique properties.

The double helix consists of two tightly associated polymer chains, each being a string of four interchangeable types of basic repeating units called nucleotides. Each nucleotide unit contains a sugar-phosphate backbone element, which carries one negative charge. Attached to each sugar is one of four types of bases. These bases are classified into two types: cytosine (C) and thymine (T) are six-membered heterocyclic, organic compounds called pyrimidines, while adenine (A) and guanine $(\mathrm{G})$ are fused five- and six-membered rings called purines. Nucleotides can be strung together in any sequence by phosphodiester bonds between the third and fifth carbon atoms of adjacent sugar rings. The length of each DNA backbone unit is about $0.34 \mathrm{~nm} .{ }^{127}$ 
The DNA double helix is mainly stabilized by hydrogen bonds between complementary bases on opposite strands forming base pairs (bp), which lie horizontally between the two spiraling strands. In the canonical Watson-Crick base pairing, adenine (A) forms a base pair with thymine $(\mathrm{T})$, as does guanine $(\mathrm{G})$ with cytosine $(\mathrm{C})$ in DNA. ${ }^{126}$ In addition, forces generated by hydrophobic effects and $\pi$-stacking, which are not influenced by the sequence of the DNA, contribute to double-helix stabilization. As the DNA strands wind around each other, two gaps between each set of phosphate backbones are generated, revealing the sides of the bases inside. There are two of these grooves twisting around the surface of the double helix: the major groove ( $2.2 \mathrm{~nm}$ wide) and the minor groove $\left(1.2 \mathrm{~nm}\right.$ wide). ${ }^{130}$ In particular, differences in width and local charge density of grooves result in the formation of specific binding sides for natural as well as synthetic polycationic agents.

\subsection{Liquid-crystalline properties of DNA in solution}

Over the past decades, extensive experimental studies have been performed on aqueous DNA solutions - water is a good solvent for $\mathrm{DNA}^{131}$ - using a multitude of experimental techniques. ${ }^{132-138}$ Due to their connectivity, stiffness, and strong electrostatic interactions, DNA molecules in solution show a wide range of complex behavior common to several synthetic and biological polyelectrolytes in the form of long rods. Their nature depends especially on the polymer concentration. At low concentrations, a DNA solution is a classical liquid with isotropically disturbed polymer chains that exhibit no preferential orientation. As the concentration gets higher, the solution passes from a dilute regime to a semi-dilute regime, where overlapping of polymer chains occurs, and to liquid-crystalline phases. The phase sequence with increasing concentration can be described as follows: The isotropic solution transforms into either blue phases or pre-cholesteric stages and then into a cholesteric phase, which turns itself into a columnar hexagonal. ${ }^{136}, 138,139$ Although DNA chain length influences phase transitions and phase boundaries, the nature and order of liquid-crystalline phases seems to remain unaffected. However, up to now no well organized liquid-crystalline phases have been obtained with long (>500nm) DNA, probably on account of the time required for the sample to organize and stabilize. ${ }^{136}$ Similar liquid-crystalline DNA organizations can be found in vivo, e.g. cholesteric DNA phases in dinoflagellate ${ }^{140}$ and hexagonal packing of DNA molecules in bacteriophages $^{141}$ and sperm heads. ${ }^{142}$ Thus, both an understanding of DNA phase 

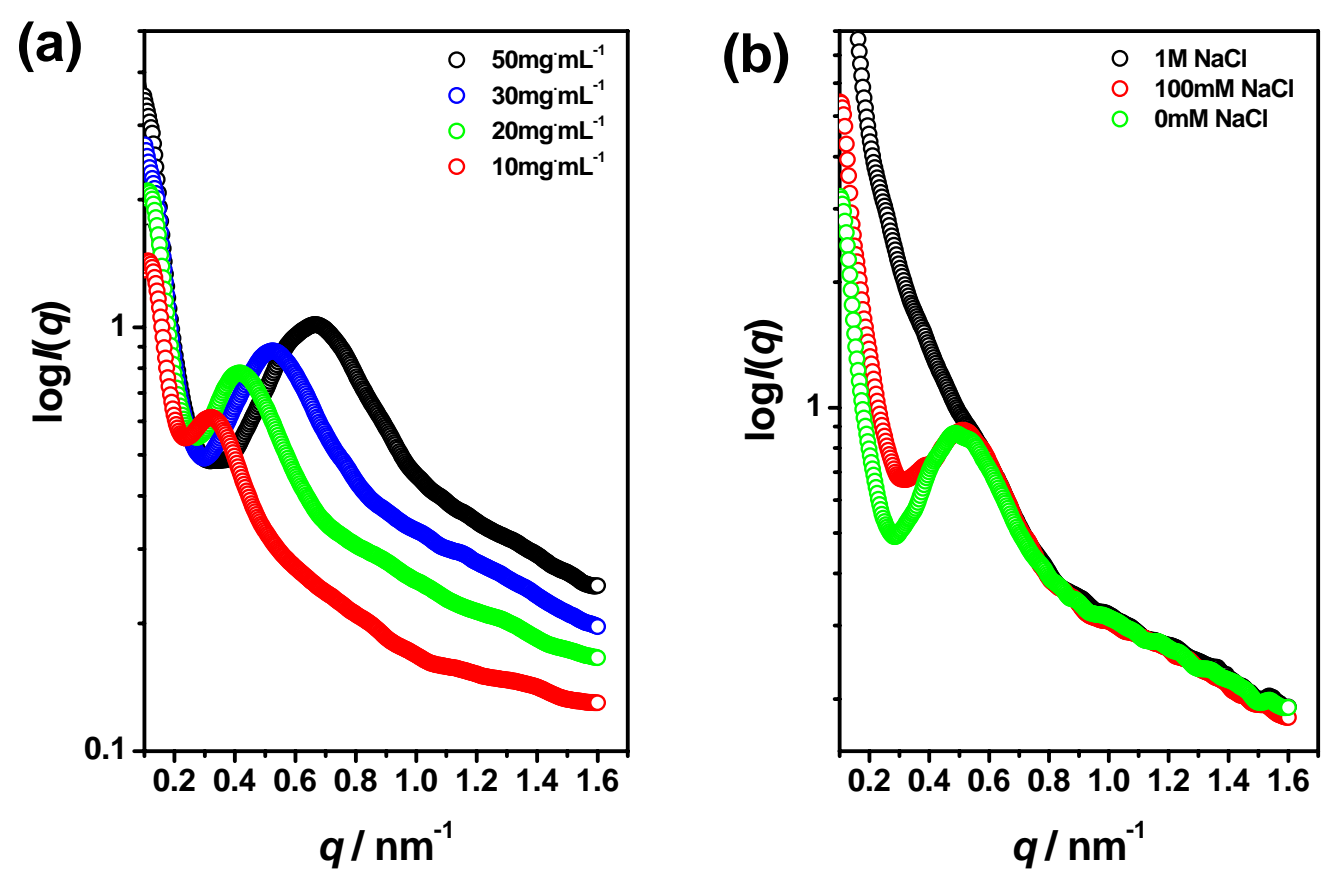

Figure 5-2: (a) SAXS intensities obtained from DNA solutions of different concentrations. (b) Effect of added salt on the scattering intensity of 30mg $\mathrm{mL}^{-1}$ DNA solution.

behavior and the knowledge of its structural features are of fundamental importance in order to understand DNA organization in vivo. Moreover, it represents an essential first step towards understanding DNA compaction.

In the framework of this thesis, lyophilized highly polymerized calf thymus DNA sodium salt (Sigma-Aldrich Chemie GmbH, Taufkirchen, Germany) and $\lambda$-DNA (Fermentas GmbH, St. Leon-Rot, Germany) are used, which are commonly employed as representative and standard polynucleotides. The average DNA chain length of the calf thymus DNA is determined to be $6 \mu \mathrm{m}$ (ca. $18000 \mathrm{bp}$ ) using gel electrophoresis (Consort, Turnhout, Belgium), whereas the monodisperse $\lambda$-DNA has a length of $16.5 \mu \mathrm{m}(48502 \mathrm{bp}$ ). The DNA is solubilized in ultrapure $18.2 \mathrm{M} \Omega \mathrm{cm}$ water (Millipore $\mathrm{GmbH}$, Schwalbach, Germany). The $\mathrm{pH}$ is controlled by adding aqueous solutions of $\mathrm{HCl}$ (Sigma-Aldrich Chemie $\mathrm{GmbH}$ ) and $\mathrm{NaOH}$ (Sigma-Aldrich Chemie $\mathrm{GmbH}$ ), respectively. Additionally, the concentration of monovalent ions $(\mathrm{NaCl}, 99.9 \%$ SigmaAldrich Chemie $\mathrm{GmbH}$ ) is adjusted if necessary. 


\subsubsection{X-ray diffraction on DNA solutions}

It is highly desirable to study DNA solutions on molecular length scales. Therefore, Xray diffraction experiments are used to follow the DNA inter-helix spacing between nearest neighbors, when increasing progressively the polymer concentration. Measurements of semi-dilute DNA solutions with concentrations ranging from 10$50 \mathrm{mg}^{-1}$ are performed in sealed quartz glass capillaries. Figure 5-2 shows exemplarily the dependence of the corrected scattering intensity on the scattering wave vector for four different concentrations. Scattering data show the existence of a single, pronounced peak in the scattering intensity at a certain position denoted by $q_{\text {DNA }}$. Assuming a Lorentzian peak shape, fits of scattering intensities are performed in order to estimate $q_{\text {DNA }}$. With increasing DNA concentration, the peak maximum shifts toward higher $q$ values and greater intensities.

Figure $5-2 \mathrm{~b}$ shows the variation of the scattering intensity exemplarily for a $30 \mathrm{mg}^{\mathrm{m}} \mathrm{mL}^{-1}$ DNA solution as a function of $q$ at different $\mathrm{NaCl}$ concentrations. Upon addition of $\mathrm{NaCl}$, the peak roughly maintains its position constant, but its intensity decreases progressively. The scattering behavior is closer to that of a neutral polymer due to progressive screening of electrostatic forces. These salt-induced changes in scattering intensity reflect the electrostatic nature of the peak. Consequently, observed peaks have to be interpreted as resulting from electrostatic interactions. These interactions impose a preferential, average distance between the charged macromolecules and lead to an "organized" structure characteristic of a cubic arrangement in the dilute regime, and a cylindrical or hexagonal packing in the semi-dilute regime. ${ }^{132,134}$ The peak position $q_{\mathrm{DNA}}$ corresponds to the average distance $d_{\mathrm{DNA}}=2 \pi / q_{\mathrm{DNA}}$ between molecules in solution. $d_{\mathrm{DNA}}$ is ranging from $9.3 \mathrm{~nm}$ for $50 \mathrm{mg}^{-1} \mathrm{~mL}^{-1}$ to $19.0 \mathrm{~nm}$ for $10 \mathrm{mg}^{-1} \mathrm{~mL}^{-1}$.

The peak position $q_{\text {DNA }}$ plotted as a function of DNA concentration is shown in Figure 5-3 in a double-logarithmic representation. Within error bars, all data points fall on the straight line shown in red. This corresponds to the relation

$$
\begin{aligned}
& q_{D N A}=a_{1} c_{D N A}^{a_{2}} ; \\
& a_{1}=(1.15 \pm 0.16) \cdot 10^{-1}, \\
& a_{2}=(0.47 \pm 0.07) .
\end{aligned}
$$

This finding is in agreement with theories for the scattering of charged polyelectrolyte solution, which predict a single peak with a position depending as $c_{D N A}^{0.33}$ on the concentration in the dilute regime and a $c_{D N A}^{0.5}$ dependence in the semi-dilute regime. ${ }^{143,144}$ The relation between peak position and concentration does not seem to 
5. DNA in Microflow

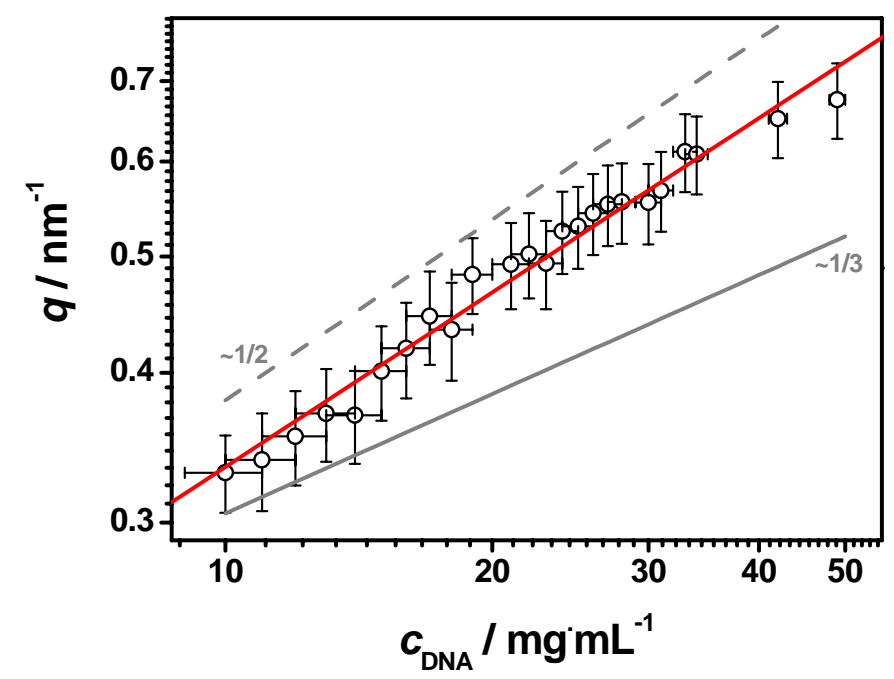

Figure 5-3: Peak position $q_{\mathrm{DNA}}$ versus DNA concentration $c_{\mathrm{DNA}}$ in double logarithmic representation. The red line is a fit to the data points according to equation (4-1). Solid and dashed gray lines represent the theoretically predicted course of dilute and semi-dilute solutions, respectively.

depend on the length of the DNA fragments used, suggesting that the position of the main maximum is independent of the molecular weight (or contour length) of DNA fragments. $^{133,135}$

More information can be obtained from an analysis of the full width at half maximum $\Delta q_{\text {DNA }}$ of the peaks obtained from Lorentz fits. In Figure 5-4, the dependence of $\Delta q_{\text {DNA }}$ on the DNA concentration is shown. Again, data points fall on a straight line in log-log representation:

$$
\begin{aligned}
& \Delta q_{D N A}=\bar{a}_{1} c_{D N A}^{\bar{a}_{2}} ; \\
& \bar{a}_{1}=(0.74 \pm 0.15) \cdot 10^{-1}, \\
& \bar{a}_{2}=(0.47 \pm 0.07) .
\end{aligned}
$$

Since peaks become broader, one would assume a change in the degree of order with increasing concentration. However, the fact that both $q_{\text {DNA }}$ and $\Delta q_{\text {DNA }}$ show the same concentration dependence leads to the expression

$$
\Delta q_{D N A}=\bar{a}_{1} / a_{1} \cdot q_{D N A} .
$$

This can be rewritten in terms of the correlation length $L_{\mathrm{c}}$ and the average molecular distance $d_{\mathrm{DNA}}$ :

$$
L_{C}=\frac{2 \pi}{\Delta q_{D N A}}=(1.55 \pm 0.16) d_{D N A}
$$




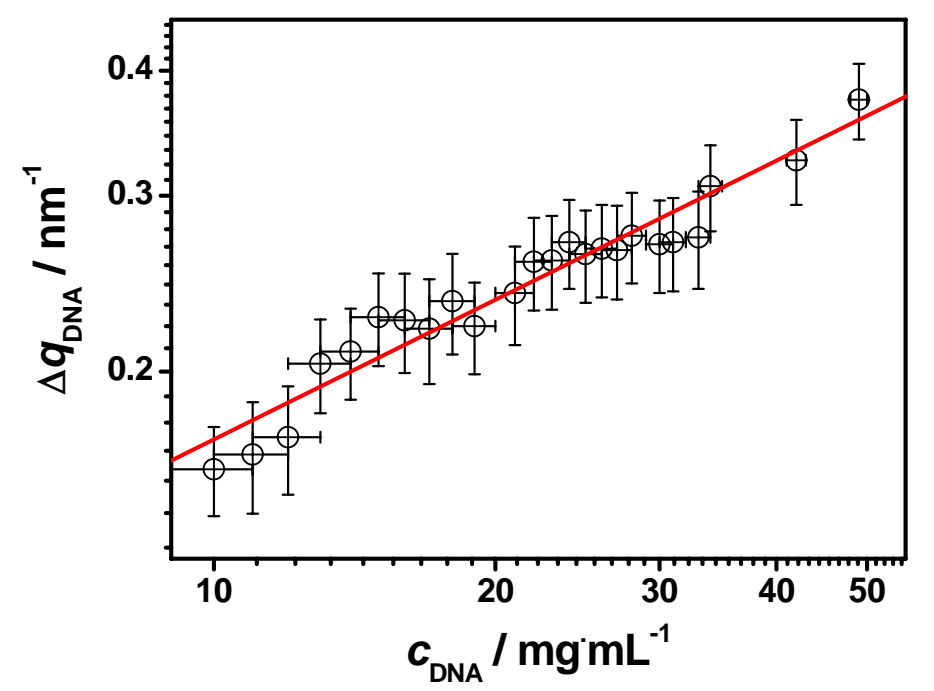

Figure 5-4: Full width at half maximum $\Delta q_{\text {DNA }}$ of the DNA peak versus DNA concentration $c_{\mathrm{DNA}}$ in double logarithmic representation. The red line is a fit to the data points according to equation (4-2).

Strikingly, this relation implies that although the correlation length is depending on the lattice spacing and therefore on the concentration, the degree of correlational order in DNA solutions is not. According to above results, the correlation between DNA molecules exceeds neighboring chains, but does not fully reach next nearest neighbors. The broadening of the peak with concentration in fact directly results from the concentration dependence of the peak position $q_{\text {DNA }}$. From the literature, comparable results are known for significantly shorter DNA molecules (40nm). ${ }^{137}$ Therefore, this result seems to be universal for the semi-dilute regime.

\subsubsection{Raman spectroscopy of DNA solutions}

In vivo DNA functionality is characterized by the complex temporal and spatial interplay of ions, metabolites, macromolecules, and macromolecular assemblies. X-ray diffraction can yield detailed information concerning the 2D and 3D organization on the molecular and mesoscale of known and unknown DNA structures and condensates. However, a full knowledge of the mechanism of the particular DNA organization and compaction will require a direct measurement of the precise conformations of molecular groups and the chemical interplay of constituents. This remains well beyond the scope of current X-ray methods. Therefore, Raman spectroscopy measurements of DNA and DNA condensates have been additionally performed. In particular, confocal 


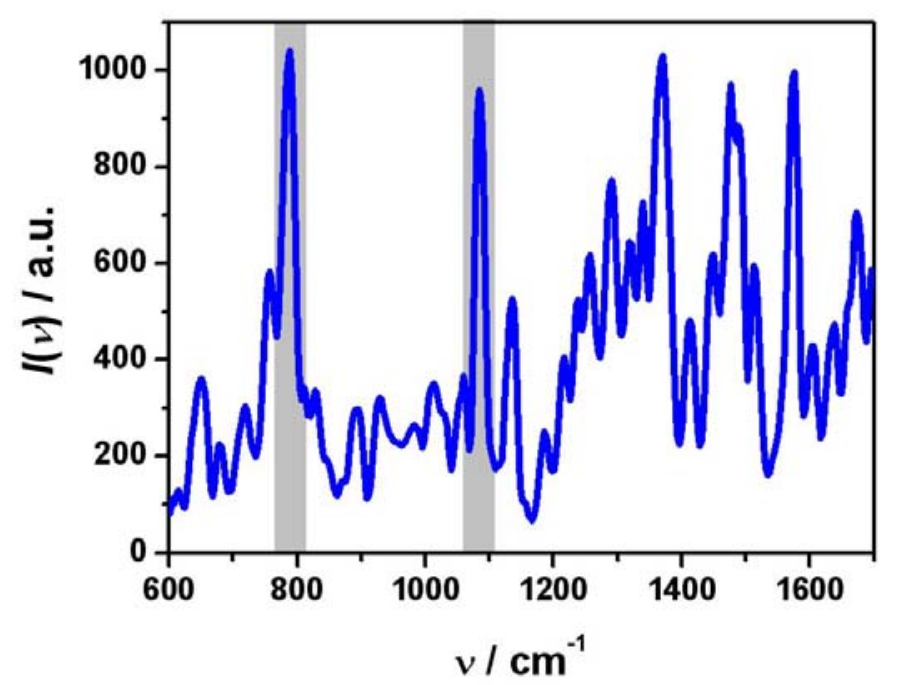

Figure 5-5: Raman spectrum of DNA. Two prominent Raman bands assigned to phosphate group vibrations are marked.

Raman microscopy enables direct sample imaging at high resolution in specific consideration of the local molecular structure without using any markers.

In a first step, it is necessary to analyze the Raman spectrum of DNA. The Raman spectrum of DNA shown in Figure 5-5 is an accumulated average of 50 exposures of $150 \mathrm{~s}$ each. The spectral resolution is $6 \mathrm{~cm}^{-1}$. Characteristic Raman bands of DNA are situated in the wave number region of $600-1700 \mathrm{~cm}^{-1}$. The Raman spectrum of DNA is rich in prominent bands. Each Raman band corresponds to specific molecular vibrations, which correspond to a change of the polarizability induced by the incident laser radiation. These vibrations are usually localized within a well defined group of atoms residing in base, sugar or phosphate moieties. According to the location of the vibrating group of atoms, Raman bands are often assigned to one of these three nucleotide entities. A reliable assignment of spectral bands to specific vibrational modes of nucleotide residues is indispensable for a successful application. This objective has been approached over the last decades using experimental and theoretical methods including comparison of Raman data with corresponding infrared spectra, isotope substitutions, and analyzing single crystals of a known structure. More details are given elsewhere. ${ }^{67}$

Due to the fact that phosphate groups are the carrier of the negative charge of DNA, Raman bands assigned to them are of special interest. The most prominent among these bands are observed at 782 and $1087 \mathrm{~cm}^{-1}$. The Raman band at $782 \mathrm{~cm}^{-1}$ is due to a vibration of the 5' C-O-P-O-C3' network of B-form DNA. It is overlapped by bands belonging to vibrations assigned to the bases thymine $\left(785 \mathrm{~cm}^{-1}\right)$ and cytosine $\left(775 \mathrm{~cm}^{-1}\right)$. The Raman band at $1087 \mathrm{~cm}^{-1}$ is due to a symmetric stretching vibration of 
the $\mathrm{PO}_{2}{ }^{-}$moiety. Both Raman bands are reliable indicator for B-form DNA. ${ }^{145-147}$ In addition, the phosphate groups give rise to Raman bands at 807 and $826 \mathrm{~cm}^{-1}$, which are both relatively weak in intensity for B-form DNA.

Vibrations localized in sugar moieties of the DNA (desoxyribose) are contributing to Raman bands at 892, 926, 1030,1058, 1413,1441, and $1477 \mathrm{~cm}^{-1}$ exhibiting very low Raman intensities. ${ }^{67,145}$

Raman bands of the bases can be mainly found in three spectral intervals: Between $600-800 \mathrm{~cm}^{-1}$ Raman bands originate from complex vibrations involving a concerted ring breathing of purine or pyrimidines residues. In the region $1130-1600 \mathrm{~cm}^{-1}$, Raman bands assigned primarily to in-plane vibrations of base residues overlap each other. Carbonyl group stretching vibrations give rise to strong Raman bands at wave numbers of $1600-1750 \mathrm{~cm}^{-1}$. $^{145}$

\subsection{DNA in microflow}

In chapter 5.2, bulk properties of DNA have been measured and discussed. However, it is of crucial interest for this study, to analyze and characterize the behavior of semidilute DNA solutions in microflow. A detailed study of this behavior provides a first step towards understanding of the more complex process of DNA condensation in microchannels. In order to improve the understanding, finite element simulations of the physical conditions inside microchannels are performed. Combining microfluidics with confocal Raman microscopy allows for a direct visualization of mutual effects of flow conditions and DNA properties. Using small angle X-ray microdiffraction, the dynamic behavior of soft matter in specific consideration of the molecular structure can be investigated. Aside from advantages such as reduced sample volumes and the possibility of high throughput, a unique feature of microfluidic setups is the steady, continuous flow, which enables in situ investigations without material damage.

\subsubsection{Influence of DNA solution viscosity on the microflow}

Performing microfluidic experiments, an important characteristic of DNA solutions with a high impact on the flow field is the viscosity. ${ }^{59}$ In order to analyze the influence of viscosity effects on flow fields in microchannels, finite element method simulations of the microflow in hydrodynamic focusing devices with crossed microchannel 
5. DNA in Microflow
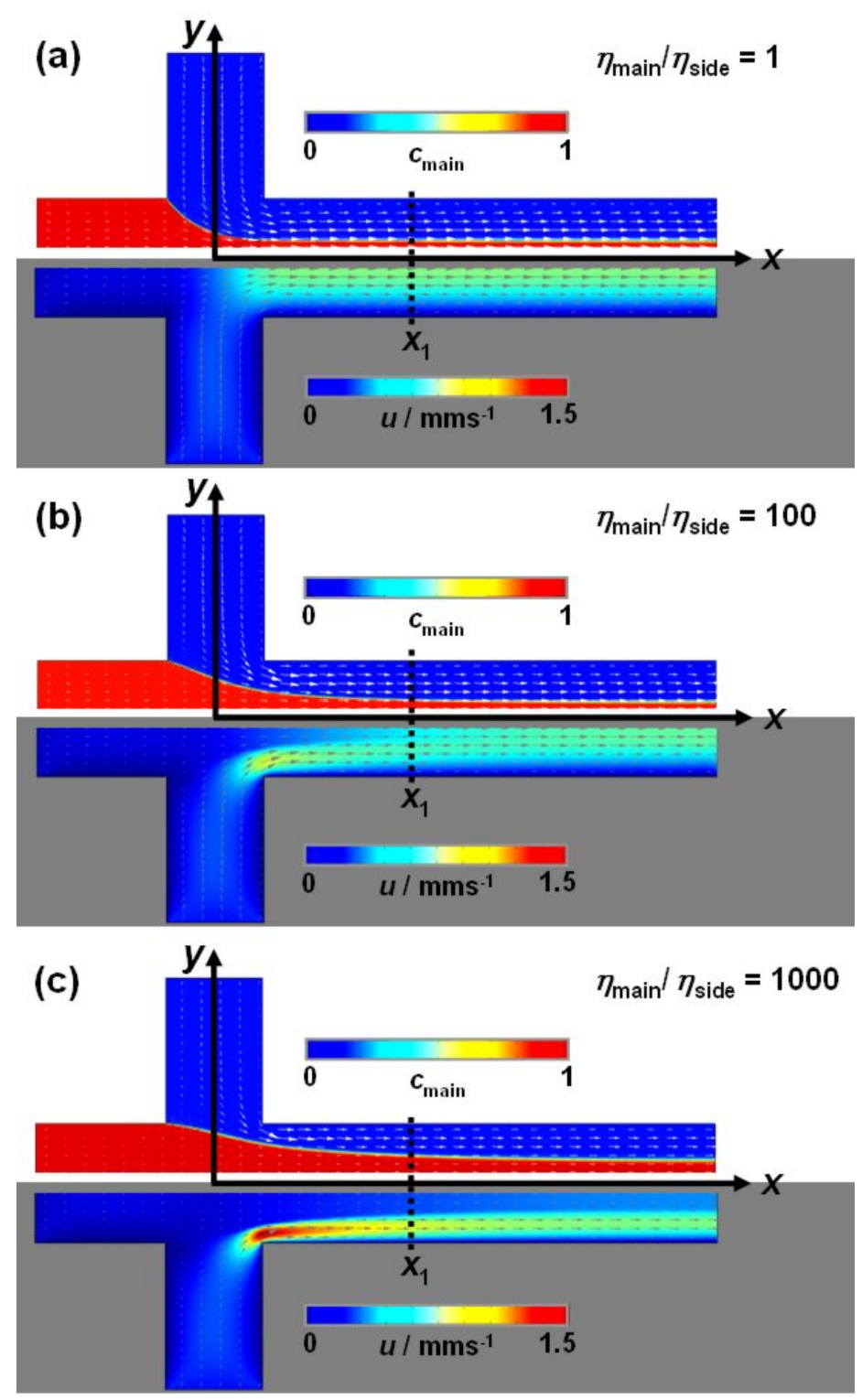

Figure 5-6: Simulation data of the concentration (upper part) and the velocity field (lower part) for three different viscosity ratios $\eta_{\text {main }} / \eta_{\text {side }}$. Corresponding simulations are scaled equally to facilitate the comparison. The position $x_{1}=300 \mu \mathrm{m}$ is annotated.

geometry are performed varying the viscosity $\eta_{\text {main }}$ of the main channel stream over three orders of magnitude. The incompressible Navier-Stokes equation coupled with the diffusion equations for the different components is solved in 2D to obtain a stationary solution (low Reynolds numbers, using about 20,000 finite elements). To allow for comparison with experiments discussed in chapter 5.3.2 and 5.3.3, a channel width of $150 \mu \mathrm{m}$ is chosen. Simulated flow velocities are $u_{\text {main }}=250 \mu \mathrm{ms}^{-1}$ and $u_{\text {side }}=2 \cdot u_{\text {main }}$. 

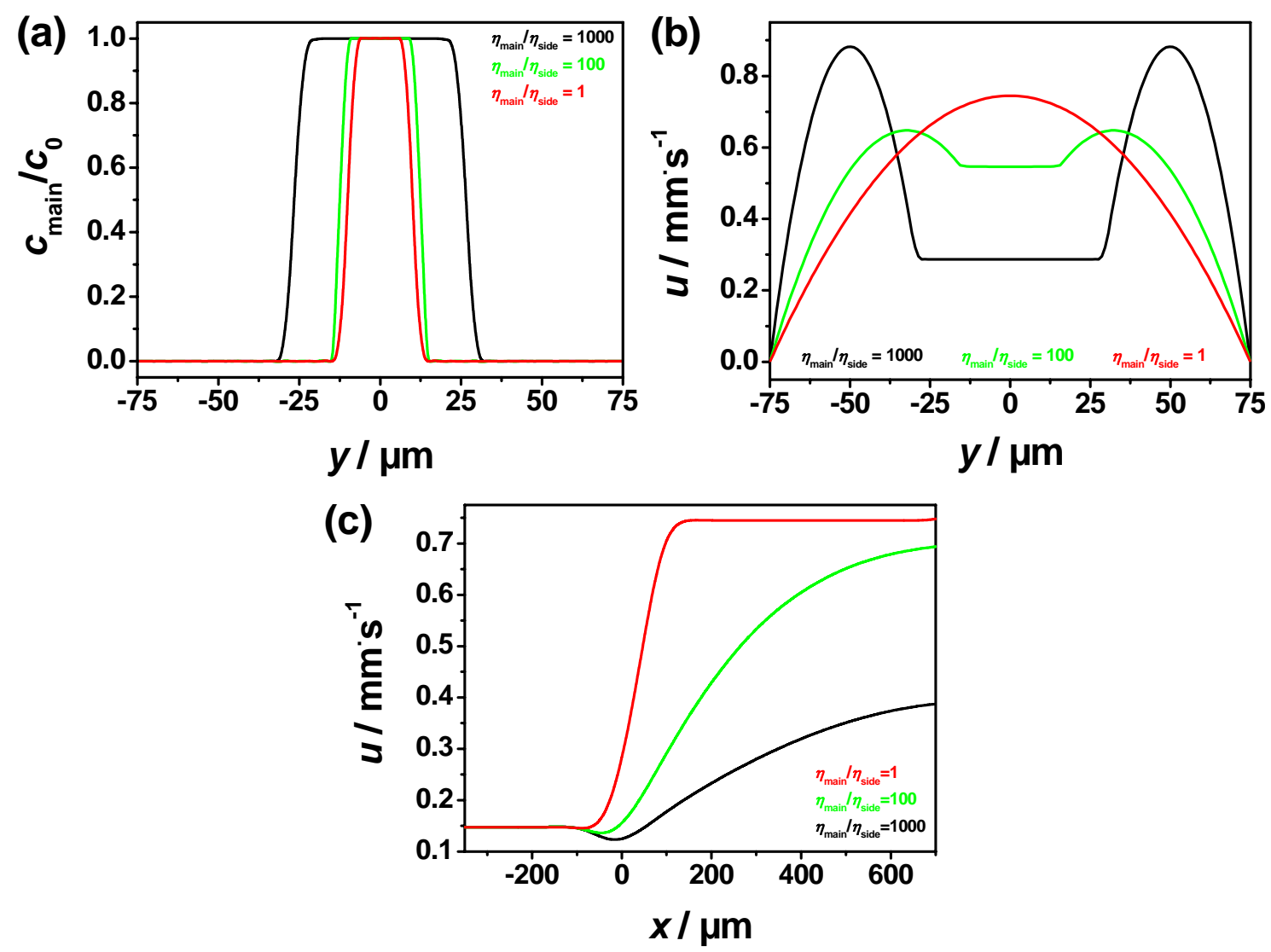

Figure 5-7: (a) Dependence of the local lateral extension of the main stream visualized by the concentration profile and (b) the flow velocity $u$ along the $y$-axis for different ratios of viscosities at a fixed position $x_{1}=$ $300 \mu \mathrm{m}$. (c) Dependence of the flow velocity $u$ along the $x$-axis $(y=0)$ for different ratios of viscosities. The velocity ratio of $u_{\text {main }} / u_{\text {side }}=1 / 2$ is kept constant.

Figure 5-6 shows simulated flow profiles and velocity fields for three different viscosity ratios $\eta_{\text {main }} / \eta_{\text {side. }}$ Owing to the microchannel symmetry, it is sufficient to examine - and therefore display - half of the channel geometry. Corresponding simulations are scaled equally to facilitate comparison. At the confluence of the three inlet channels, flow patterns are interfering and focusing of the main liquid stream as well as a simultaneous acceleration of the flow can be observed. Although flow velocities of all three inlet channels remain unchanged, depending on the ratio of viscosity $\eta_{\text {main }} / \eta_{\text {side }}$ significant differences in flow profiles are observable.

Figure 5-7a shows the dependence of the local lateral extension of the main (DNA) stream - visualized by the concentration profile (left) - and the flow velocity (right) along the $y$-axis for different ratios of viscosities at a fixed position $x_{1}=300 \mu \mathrm{m}$. For a purely aqueous system, the viscosity in the center stream and the side streams is equal $\left(\eta_{\text {main }} / \eta_{\text {side }}=1\right)$. Therefore, all incoming streams behave as a single flow. In this case, a 
parabolic velocity profile perpendicular to the flow direction can be obtained in the microchannel as expected. Due to the chosen velocity ratio of $u_{\text {main }} / u_{\text {side }}=1 / 2$, approximately $1 / 5$ of the main channel is occupied by the center stream as can be seen from the concentration profile in Figure 5-7a.

In contrast to a purely aqueous system, for highly viscous systems a strikingly different behavior can be observed. With increasing viscosity, the flow velocity of the center stream is less influenced by the inflowing side streams. This reduced influence is reflected in the concentration profile, which exhibits a larger lateral extension of the center stream (Figure 5-7a). Consistently, flow velocities of the side streams are dramatically increased. Parabolic flow profiles developing in both side streams can be observed perpendicular to the flow direction. Interfaces between the side and the main stream act like additional soft walls and side streams flow as if in channels of smaller width. Moving down the main channel, a parabolic flow profile throughout the whole channel is expected to eventually evolve.

In Figure 5-7b, line scans along the center of the main channel parallel to the flow direction are shown. For $\eta_{\text {main }} / \eta_{\text {side }}=1$, a strong acceleration of the flow velocity occurs, which is limited to the microchannel intersection. After passing it, the flow velocity remains constant. However, with increasing viscosity of the solution in the main channel, the influence of the side streams on the main stream is dramatically reduced. Consequently, the velocity profile parallel to the flow direction in the center of the main channel increases only very slowly and the acceleration region expands several orders of magnitude. Depending on the ratio of $\eta_{\text {main }} / \eta_{\text {side, }}$ an acceleration is noticeable several $1000 \mu \mathrm{m}$ downwards the outlet channel. Locating DNA molecules in the main stream, this long-lasting acceleration phase in the hydrodynamic focusing device can be exploited to align DNA molecules in a tunable way.

\subsubsection{Confocal Raman microscopy of DNA in microflow}

To experimentally access conditions in the microchannel, confocal Raman measurements are performed. A center stream of semi-dilute DNA solution with a mean velocity of $u_{\text {main }}=250 \mu \mathrm{ms}^{-1}$ is hydrodynamically focused by two symmetrical side streams of water with $u_{\text {side }}=2 \cdot u_{\text {main }}$. Confocal Raman images of the DNA jet in Figure 5-8 (upper part) are recorded in the middle-plane of the microfluidic cell by lateral scanning using the avalanche photodiode detector at a specific wave number of $v=1576 \mathrm{~cm}^{-1}$. At this wave number, the Raman spectrum of DNA shows a prominent peak, which arises from vibrations of the bases guanine and adenine. 


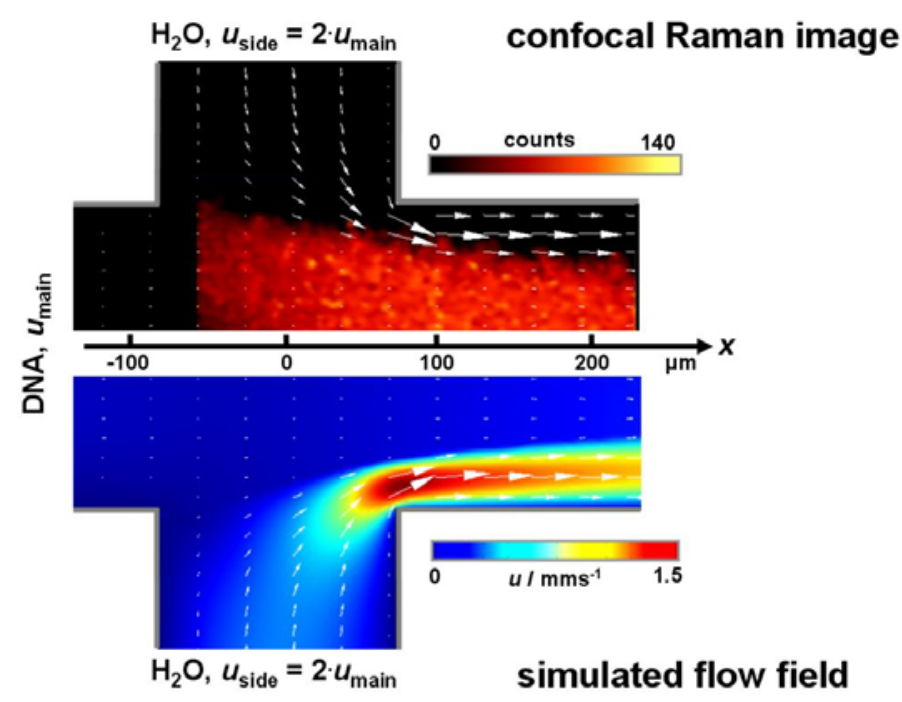

Figure 5-8: Confocal Raman image (top) of a hydrodynamically focused DNA stream recorded at a fixed wave number of $v=1576 \mathrm{~cm}^{-1}$. For direct comparison the simulated velocity profile is overlaid and the modeled flow field (bottom) shown.

Performing Raman analyses, two competing effects have to be considered. On the one hand, the intensity of the Raman signal increases with concentration. On the other hand, DNA solutions exhibit a self-fluorescence, which is problematic at higher concentrations. Therefore, a semi-dilute DNA solution with a concentration of $10 \mathrm{mg} \mathrm{mL}^{-1}$ is used.

In order to quantify the influence of viscosity effects on the flow fields in the microchannels, finite element simulations are performed, and results are compared to the experiment. Apart from the viscosity of the DNA solution, all simulation parameters such as channel geometry and flow rates are known. The viscosity of the DNA solution is used as a fit parameter in the simulations to match experimental results, i.e. the shape of the hydrodynamically focused center stream. The viscosity is fitted to $\eta_{1} \approx 10^{2} \cdot \eta_{2}=10^{2} \cdot \eta_{\text {water }}=89.1 \mathrm{mPas}$ ( $\eta_{\text {water }}=0.891 \mathrm{mPa}$ ). This is in good agreement with the result obtained from bulk measurements $\eta_{\mathrm{DNA}} \approx 0.1 \mathrm{~Pa}$ s using an Ubbelohde-viscometer.

The simulated flow field is shown in Figure 5-8. For direct comparison with experimental results, the modeled velocity profile in the hydrodynamic focusing device is overlaid to the Raman image. Experiment and simulation show good agreement. It is possible to achieve the actual shape of the hydrodynamically focused DNA solution with high precision as shown in Figure 5-8. The stream profile of the DNA solution shown in Figure 5-8 displays a significant broadening in shape due to the high solution viscosity. The results demonstrate the consistency of bulk viscosimetry, microfluidics 
experiments, and finite element simulations. In turn, this technique provides a method which enables us to evaluate the viscosity of minute quantities of very viscous fluids.

\subsubsection{X-ray microdiffraction analysis of DNA in microflow}

Besides visualization and simulation of flow profiles, analysis of the influence of flow conditions on DNA molecules inside hydrodynamic focusing devices at a molecular length scale is highly desirable. Therefore, small angle X-ray microdiffraction is utilized to illuminate conformational details of the DNA molecular ordering at different positions in the channels with high resolution. In particular, X-ray experiments profit from the alignment of DNA molecules.

A center stream of semi-dilute DNA solution with a mean velocity of $u_{\text {main }}=250 \mu \mathrm{m} \cdot \mathrm{s}^{-1}$ is hydrodynamically focused by two symmetrical side streams of a $67 \%$ aqueous glucose solution with $u_{\text {side }}=4 u_{\text {main }}$. A DNA correlation peak at $q_{\mathrm{DNA}}=0.498 \mathrm{~nm}^{-1}$ is measured in the inlet channel at $x=-200 \mu \mathrm{m}$ (Figure 5-9a). According to results in chapter 5.2.1, the concentration of the DNA solution is determined from $q_{\mathrm{DNA}}$ to be $23 \mathrm{mg}^{-1} \mathrm{~mL}^{-1}$. The inter-axial spacing of DNA molecules is $d_{\mathrm{DNA}}=12.6 \mathrm{~nm}$. Glucose solutions are chosen for hydrodynamic focusing of the DNA stream in order to establish a viscosity ratio of the solutions in the main and the side channels of $\eta_{\text {main }} / \eta_{\text {side }} \approx 15$.

Scans of the intensity along the azimuthal angle $\chi$ on the ring of the $q_{\text {DNA }}$ peak at different channel positions $x$ are shown in Figure 5-9b. Owing to the symmetry of $I(\chi)$, it is sufficient to display the range $\chi=0-180^{\circ}$. Maximal intensities can be observed at azimuthal angles of $\chi_{\max } \approx \pm 90^{\circ}$. The degree of orientation can be quantified as the full width at half maximum $\Delta \chi$ of a Gaussian fit of the azimuthal peaks. In Figure 5-9c, the dependence of $\Delta \chi$ from the position in the main channel is compared to the local strain rate $\dot{\varepsilon}=\partial u / \partial y$, which is calculated from the simulated velocity field shown in Figure 5-7b.

Owing to the highly concentration dependent viscous flow, DNA molecules show a weak pre-elongation along the flow direction in the inlet channel. Starting at $x \approx-100 \mu \mathrm{m}$, a continuous decrease of $\Delta \chi$ is observable. This corresponds to a steady increase in the orientation of DNA molecules. According to the course of the strain rate, this is only expected for $x>-50 \mu \mathrm{m}$. Moreover, an initial decrease in the strain rate owing to obstruction effects induced by hydrodynamic focusing and therefore a 

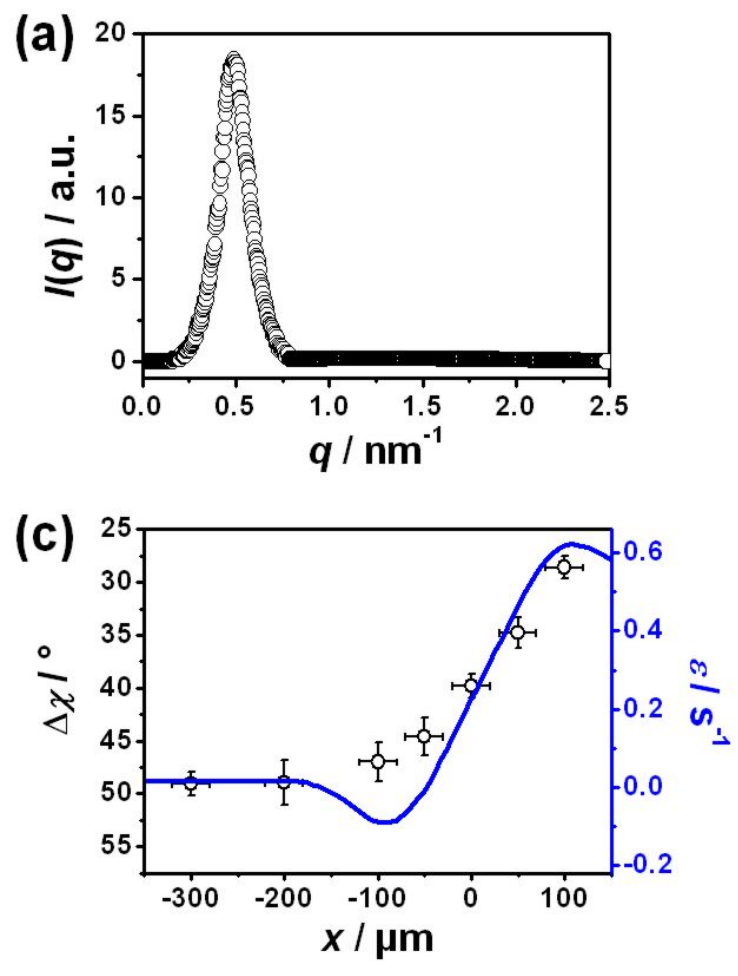

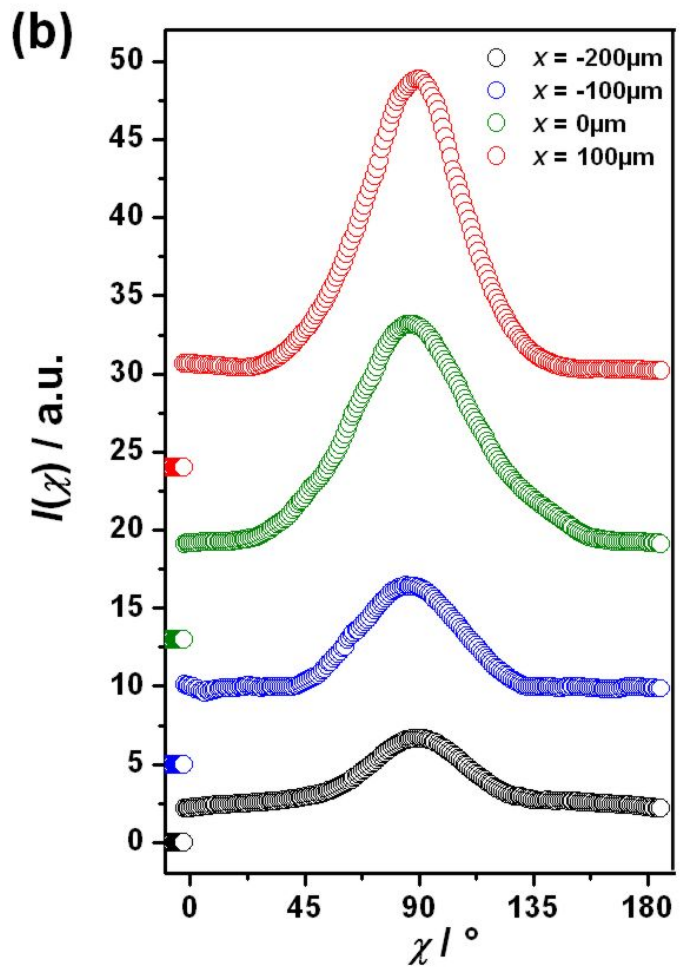

Figure 5-9: (a) DNA correlation peak. (b) Intensity modulation of the DNA correlation peak (scans of the azimuth angle $\chi$ ) at different positions $x$. (c) Dependence of the standard deviation $\Delta \chi$ on the position along the $x$ axis compared to the strain rate $\dot{\varepsilon}$ obtained from finite element simulations.

decrease in orientation is expected. This is reflected in the simulation data shown in Figure 5-9c. Both, the early onset of the increase in orientation and the absence of an initial decrease in orientation can be attributed to the fact that the long chain DNA molecules in semi-dilute solutions are entangled. In the channel intersection area, a significant increase in orientation is observed. This behavior quantitatively corresponds to the calculation of the strain rate as provided from finite elements simulations. One can clearly see how appropriate flow fields owing to hydrodynamic focusing inside microchannel systems are for successive stretching and aligning of long chain DNA molecules in solutions. 


\subsection{Conclusions}

As an essential first step towards understanding DNA compaction, semi-dilute DNA solutions have been studied in bulk. SAXS data show that the short-ranged order for DNA rods has a correlation length slightly exceeding first neighbors. In addition, the DNA Raman spectrum has been recorded and analyzed. In order to analyze viscosity effects on flow fields in microchannels, a finite element modeling of the experimental conditions in the microchannel device has been performed. Varying the ratio of main channel to side channel viscosity, $\eta_{\text {main }} / \eta_{\text {side, }}$ simulations show dramatic effects on the flow profiles. Increasing $\eta_{\text {main }} / \eta_{\text {side }}$ yields a strong acceleration of side stream flow velocities, which in turn strongly affects local concentrations. Moreover, it is an important result that the acceleration region can be expanded several orders of magnitude by controlling $\eta_{\text {main }} / \eta_{\text {side }}$. This effect has been exploited to align DNA molecules situated in the main stream. The orienting influence of flow conditions has been directly quantified by SAXS measurements in flow. The fact that simulations and experimental results obtained by confocal Raman imaging of the DNA jet are in good agreement demonstrates the consistency of microfluidic experiments and finite element modeling. In turn, this technique provides a method to evaluate the viscosity of minute quantities of highly viscous fluids. 
6. Dendrimers

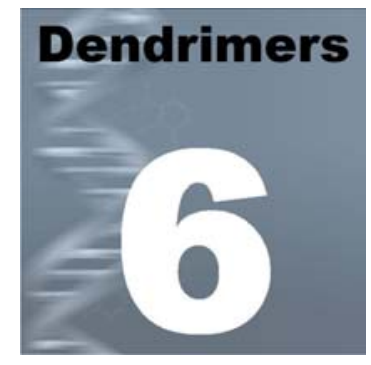

\section{DENDRIMERS \\ Characterizing Compaction Agents}

Dendrimers are a unique class of precisely engineered, highly branched, synthetic macromolecules $^{31,32}$ that can be easily tailored to act as highly specific binders of various biological and chemical substances. This has led to multifold applications ranging from surface coatings, ${ }^{148}$ catalysis, ${ }^{149}$ scaffolds for quantum dots, ${ }^{150}$ plastic additives and property modifiers, ${ }^{151,152}$ to the inhibition of HIV infection in primate studies. ${ }^{153}$ In particular as compaction agents for DNA condensation, dendrimers attract wide interest from physicists, chemists, and biologists. The 3D nanosized structure of the high generation dendrimers makes this class of molecules suitable as mimics of proteins. Furthermore, dendrimers represent one of the most efficient polymeric gene carriers. ${ }^{44-52}$ In order to understand dendrimer induced DNA compaction, it is of crucial importance to first characterize the compaction agents in detail. This chapter is dedicated to this issue. 


\subsection{General structure and characteristics}

Contrary to other hyper-branched polymers, which are produced by one-step reactions leading to irregular, polydisperse molecules with non-ideally defined structures, ${ }^{154}$ dendrimers are synthesized by step-wise reaction sequences. This systematic build-up leads to well defined molecules with a high structural and chemical homogeneity. ${ }^{33}$ The unique architecture of dendrimers features iterative attaching of polymeric subunits with a certain chain length $P$ and chemical valence $f_{\mathrm{b}}$ around an initiator core of a certain chemical valence $f_{\mathrm{c}}$. Figure 6-1 shows a schematic representation of the most commonly used case of $f_{\mathrm{c}}=4$. Additional branching units generally have a functionality of $f_{\mathrm{b}}=2$ and the same molecular weight $M_{\mathrm{w}}$. However, successive attachment of branches is not limited to this simple progression. When additional branches have been added at each available chain end of the dendrimer exterior, another "layer" or "generation" is completed. Thus, the size of the molecules is determined by the number $G$ of generations present in the polymers. For dendrimers with a regular architecture, the total number of segments $N_{\text {tot }}$ can be expressed as a function of these quantities: ${ }^{39}$

$$
N_{\text {tot }}=1+\frac{f_{c}}{f_{b}-1}\left(f_{b}^{G+1}-1\right)
$$

Depending on their generation, they possess diameters up to tens of nanometers and are therefore capable of self-organizing into superstructures. ${ }^{34,} 35$ However, there is a natural limit in the maximum generation number that can be completed chemically. This is owing to the fact that the high degree of branching causes the number of monomers $N$ to grow exponentially with distance from the core whereas the available volume increases only cubically. ${ }^{155}$ This issue raises the question of whether polymeric arms emanating from the central core are highly stretched in order to increase the available volume, or if there is significant back folding of terminal segments towards the center of the dendrimer.

The organization and distribution of internal segment densities, in particular the location and accessibility of terminal groups, play a key role in understanding not only the molecular properties themselves, but also their interactions with other molecules. Much of the progress in understanding the underlying physics controlling dendrimer properties has come from investigations via computer simulations. Most of these simulations seem to agree that back folding of dendrimer end groups occurs, leading to a distribution of terminal segments throughout the whole molecule. ${ }^{156-158}$ As a consequence, most simulations support a radial segment density profile having a 


\section{Dendrimers}

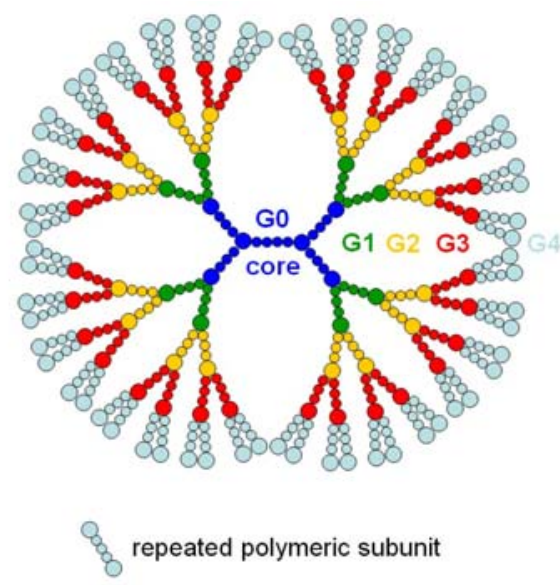

Figure 6-1: Schematic representation of the dendrimer architecture for the case of a tetrafunctional core $f_{\mathrm{c}}=4$ and bifunctional branches $f_{\mathrm{b}}=2$.

maximum at the core and decreasing to the surface. ${ }^{156-158}$ This is supported by density profiles obtained from small angle X-ray scattering data of PAMAM dendrimers generation $2-10^{159,160}$ and in particular by contrast variation small angle neutron scattering experiments using labeled end groups in a fourth-generation dendrimer. ${ }^{161}$ The hybrid character of dendrimers between colloidal entities on the one hand and their polymeric structure on the other hand is best appreciated by describing the overall shape of dendrimers. An analysis of SAXS data reveals that - at least for $G \geq 3$ dendrimers exhibit a rather compact, globular shape. It has been shown, that the most consistent description of experimental data has been achieved by modeling dendrimers as globular particles with a fuzzy surface. ${ }^{160}$

In the present study, two types of amino terminated dendrimers of different generations are used: poly(amido-amine) (PAMAM) dendrimers $(99.9 \%$ pure, Sigma-Aldrich Chemie GmbH, Taufkirchen, Germany) and poly(propylene-imine) (PPI) dendrimers (99.9\% pure, Sigma-Aldrich Chemie $\mathrm{GmbH}$ ), the two by far most commonly used types of dendrimers. These two types of dendrimers have been chosen due to their commercial availability with a wide variety of generations and peripheral functionalities as well as their relevance as scaffolds for effective DNA transfection. ${ }^{162}$ Figure 6-2 shows a schematic representation of PAMAM generation 3 and PPI generation 5 dendrimers and their repeated polymeric subunit. As shown in Figure 6-2, there is an inconsistency in literature in counting generations for these two types. Important in the frame of this thesis is the fact that this leads to different equations accounting for the number of total segments $N_{\text {tot }}$ in respect to the generation number $G$ :

$$
\begin{aligned}
N_{\text {tot }}^{\text {PAMAM }} & =2^{G+3}-3 \\
N_{\text {tot }}^{P P I} & =2^{G+2}-3
\end{aligned}
$$



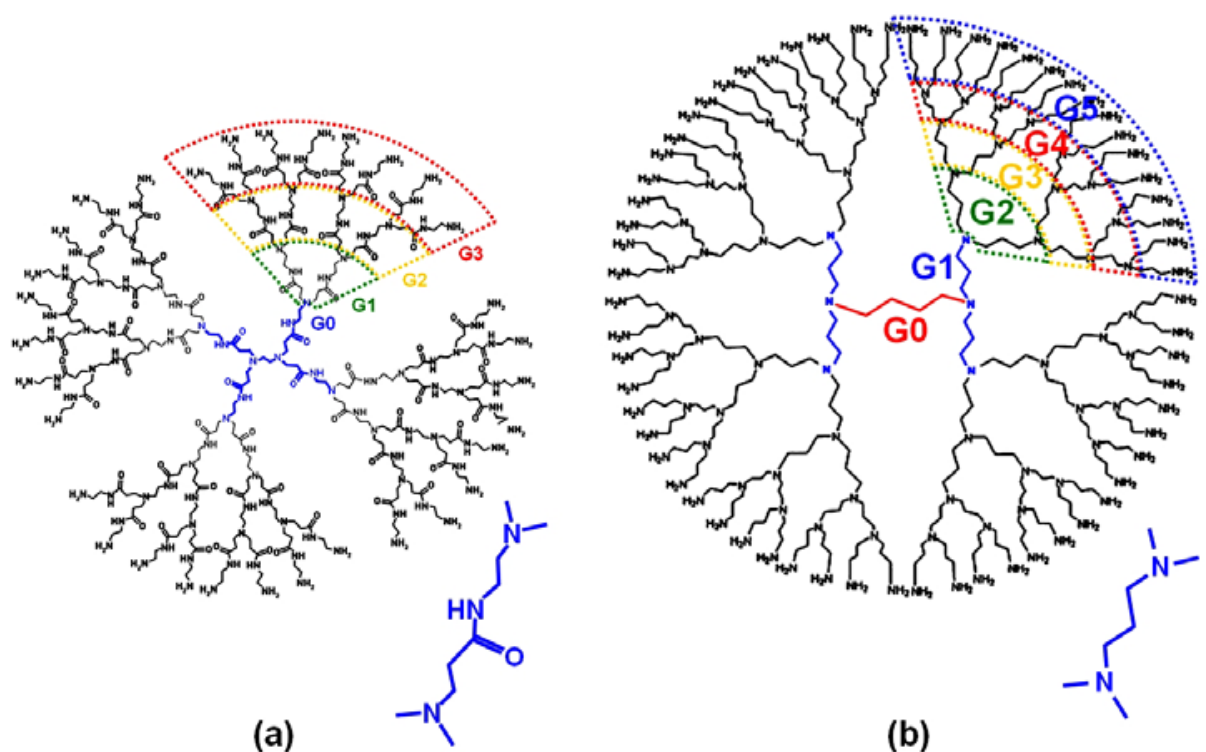

(b)

Figure 6-2: Schematic representation of (a) a poly(amido amine) dendrimer generation 3 (PAMAM 3) and (b) a poly(propylen imine) dendrimer generation 5 (PPI 5). Repeated polymeric subunits are depicted.

Up to this point, the discussion of dendrimers has implicitly been limited to neutral dendrimers excluding electrostatic effects. However, PAMAM and PPI dendrimers are polyelectrolytes comprising a multiplicity of potentially charged primary and tertiary amine groups which are of crucial importance for most applications.

In general, charged groups strongly affect polymeric conformation and size. Higher molecular charge results in electrostatic repulsion between the like-charged polymeric segments. Accordingly, a more expanded and more hydrated polymer conformation is adapted as has been demonstrated for linear ${ }^{163}$ and star-like ${ }^{164}$ polymers. Similar effects are expected for dendrimers. Due to their unique properties resulting from their precise architecture, dendrimers should respond to superimposed electrostatics in a very predictable manner.

A very simple method of influencing the charge of polyelectrolytes with high significance in biological systems and for various applications is the variation of the $\mathrm{pH}$ value of their solvent. All reported simulations advise that PAMAM as well as PPI dendrimers should exhibit conformational changes due to different degrees of protonation at different $\mathrm{pH}$ values and/or ionic strengths. ${ }^{40,43,165,166}$ However, so far only one experimental study has been performed on this topic. PAMAM dendrimers generation 8 have been analyzed utilizing SANS and no change of dendrimer size upon variation of the $\mathrm{pH}$ value and for the ionic strength has been observed. ${ }^{38}$ Therefore, there is a discrepancy between theory and experiments making the charge-induced 


\begin{tabular}{|c|c|c|c|c|}
\hline \multirow{2}{*}{ G } & \multirow{2}{*}{ No. of atoms } & \multirow{2}{*}{$\boldsymbol{M}_{\mathbf{w}} / \mathbf{g} / \mathbf{m o l}$} & \multicolumn{2}{c|}{$\begin{array}{c}\text { No. of amino groups } \\
\text { primary }\end{array}$} \\
\hline \multicolumn{5}{c|}{ tertiary } \\
\hline 3 & 1092 & 6909 & 32 & 30 \\
\hline 6 & 9156 & 58048 & 256 & 254 \\
\hline 8 & 36804 & 233383 & 1024 & 1022 \\
\hline \multicolumn{5}{|c|}{ PPI dendrimers } \\
\hline 3 & 354 & 1684 & 16 & 14 \\
\hline 4 & 706 & 3514 & 32 & 30 \\
\hline
\end{tabular}

Table 6-1: Number of atoms, primary and tertiary amino groups for PPI and PAMAM dendrimers used.

dendrimer swelling and the $\mathrm{pH}$-dependant conformational behavior a subject of considerable debate. ${ }^{38-43}$

Additional experimental results are needed to figure out whether the phenomenon of charge-induced dendrimer swelling is absent altogether. To analyze dendrimer properties, small angle X-ray scattering (SAXS) experiments on PAMAM dendrimers generation 3, 6 and 8 and PPI dendrimers generation 3 and 4 have been performed.

\subsection{X-ray diffraction of dendrimer solutions}

Molecular sizes, shapes, and intermolecular interferences contribute to scattering curves obtained from a polymer solution. According to equation (2-7), the experimentally recorded scattering intensity $I(q)$ consists of contributions of the form factor of the scattering entities, $F(q)$, and the structure factor, $S(q)$. The latter accounts for inter-particle interactions. For sufficiently diluted solutions, inter-molecular correlations can effectively be ignored and $S(q)$ approaches unity. Thus, it becomes possible to obtain the scattering intensity at scattering vectors $q$, whose coherent part is directly proportional to the form factor of the observed macromolecules,

$$
I(q) \underset{c \rightarrow 0}{\sim} N F(q) .
$$

For semi-dilute solutions however, $S(q) \approx 1$ is no longer true for very low $q$ values leading to a slight depression of $I(q)$. The advantage of experiments in the semi-dilute regime over a more concentrated one is that inter-particle interactions, which affect changes in the scattering function, are unlikely to exist. Therefore, the form factor $F(q)$ is almost unchanged but the quality of the scattering data and is simultaneously 


\section{Dendrimers}

increased due to the higher concentration. Solutions with a polymer mass fraction of $1 \%$ can be considered to be in the dilute regime, while mass fractions of $5 \%$ exhibit a small downturn at low- $q$ with no significant effect for $q>0.5 \mathrm{~nm}^{-1} .159$

The overall particle size is typically expressed by the radius of gyration $R_{\mathrm{g}}$. $R_{\mathrm{g}}$ gives measure of the mean square distance of scattering centers from the molecule's center of mass,

$$
R_{g}^{2}=\frac{1}{N}\left\langle\sum_{i=1}^{N}\left(r_{i}-r\right)^{2}\right\rangle .
$$

In the above equation, $r_{i}, i=1 \ldots N$, denote the positions of the $N$ monomers comprising the polymer. $r$ is the position of the center of mass. The brackets denote averaging over all measurable microscopic states, each weighted with the corresponding Boltzmann factor for the energy of the instantaneous configuration. For spherical particles, the radius of gyration is connected to the radius of the spheres $R$ according to the following equation: $^{167}$

$$
R=\sqrt{\frac{5}{3}} R_{g}
$$

The most common methods for determining the radius of gyration are graphical methods. There are primarily two graphical methods known as Guinier and Zimm plot, respectively. ${ }^{168}$ Zimm fits become accurate for systems that can be described as long Gaussian chains. Guinier fits represent a method suited for objects, which can be described as spheres. Based on the dendrimer molecular architecture, it is reasonable to assume that dendrimers are rather spherical objects. Therefore, their particle scattering factor in the low $q$ limit $\left(q R_{\mathrm{g}}<<1\right)$ can be approximated by the Guinier approximation for the scattering intensity ${ }^{69,159,167}$ with the functional form:

$$
I(q)=a_{1}+a_{2} \exp \left(-q^{2} R_{g}^{2} / 3\right) .
$$

Performing fits to scattering profiles in the low $q$ limit $\left(q R_{\mathrm{g}}<<1\right)$ with above equation yields the radius of gyration $R_{\mathrm{g}} . a_{1}$ and $a_{2}$ are used to account for the absolute scale and the baseline of experimental data.

However, this way of determining $R_{\mathrm{g}}$ is limited when experimental data for sufficient low $q$ values are not available. In this study, the smallest accessible region of the particle scattering term varies from $q_{\min } R_{\mathrm{g}} \approx 0.22$ for PPI dendrimers generation 3 to $q_{\text {min }} R_{\mathrm{g}} \approx 0.9$ for PAMAM dendrimers generation 8 . Therefore, estimating $R_{\mathrm{g}}$ by the Guinier approximation, which is based on expansions around $q=0$, is assumed to be increasingly inappropriate with increasing dendrimer size. Consequently, the Guinier 


\section{Dendrimers}

approximation is only used for the smaller dendrimers PPI generation 3, 4 and PAMAM generation 3. For dendrimers of higher generations, a different approach has to be used, which is described below.

Owing to their precise architecture, dendrimers are monodispersely synthesizable. The degree of dendrimer polydispersity has been measured by atomic force microscopy to be less than 1.08 for generations 5-10. ${ }^{169}$ Accordingly, no polydispersity of the system has to be considered. Different dendrimeric entities are contributing to the dendrimer scattering spectra at $q$ ranges corresponding to their length scales. The overall dendrimer shape on larger length scales determines the scattering intensity in the low $q$ region. Beside this, the loose, polymeric character of dendrimers gives rise to internal density variations with a correlation length $\xi$. The so-called "blob scattering" originating from these contributes significantly to the overall scattering intensity at larger scattering vectors. Accordingly, the dendrimer form factor $F(q)$ can be described by the sum of scattering arising form the overall shape, $F_{\text {shape }}(q)$, and the blob scattering contribution, $F_{\text {blob }}(q):{ }^{170}$

$$
F(q)=F_{\text {shape }}(q)+a_{\text {rel }} F_{\text {blob }}(q)
$$

$a_{\text {rel }}$ gives the relative weight of the two terms.

$F(q)$ is the Fourier transform of the density correlation function $\chi(r)$. Taking the sum of both terms in equation (6-7) instead of their convolution is strictly valid only if the $q$ ranges of both scattering contributions are clearly separated, allowing to neglect interference terms. According to the model introduced by Beaucage, ${ }^{171}$ this can be ascertained by replacing $q$ by $\bar{q}$ in $F_{\text {blob }}(q)$ :

$$
\bar{q}=\frac{q}{\left(\operatorname{erf}\left(q R_{g} / \sqrt{6}\right)\right)^{3}} .
$$

This results in a cut-off to low $q$ values, where shape contributions become dominant. A so-called 'blob' is a spherical volume with a radius $\xi$. Within a blob, sections of the dendrons have to be described as self-avoiding (sub) walks and excluded volume interaction has to be taken into account. Accordingly, scattering from length scale $r \leq \xi$ is therefore analogous to that of a semi-dilute polymer solution. Other than in semidilute solutions, where the blob size depends only on the polymer concentration, in the Daoud-Cotton model, ${ }^{172}$ which was originally developed for star polymers, it is postulated that $\xi$ is expected to increase with radial distance from the center. However in the $q$ range of interest, contributions from the largest, outermost blobs should significantly dominate the scattering signal. Therefore, the $r$ dependence of $\xi$ can be neglected. ${ }^{160}$ 
6. Dendrimers

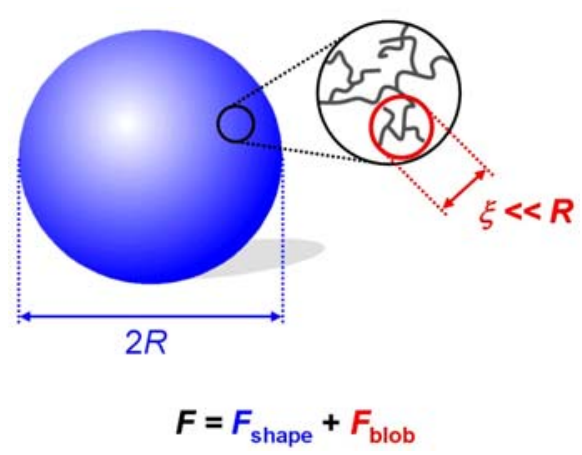

Figure 6-3: Schematic representation of the two contributions $F_{\text {shape }}$ and $F_{\text {blob }}$ stemming from the overall dendrimer shape and from the loose polymeric character of dendrimers, respectively.

$F_{\text {blob }}(q)$ describes density variations on length scales smaller than the correlation length $\xi$ of internal density variations. On length scales larger than $\xi, \chi(r)$ is equal to zero. Determining $F_{\text {blob }}(q)$, the integration in equation (6-9) is therefore limited to $r \leq \xi$,

$$
F_{b l o b}(q)=4 \pi \int_{0}^{\xi} r^{2} \gamma(r) \frac{\sin (q r)}{q r} d r .
$$

The density correlation function can be described by a power-law dependence, whose exponent can be derived from the Flory-Huggins parameter $v=3 / 5$ for good solvent conditions, which are fulfilled for the DNA-water system: ${ }^{173}$

$$
\gamma(r) \sim r^{\mu-2} ; \mu=v^{-1}-1 .
$$

In order to obtain an analytic expression for $F_{\text {blob }}(q)$, the factor $\exp (-r / \xi)$ is introduced into the integral in equation (6-9) as cut-off and the integration is extended to infinity. Taking into account equations (6-8), (6-9), and (6-10), the following expression can be derived for the scattering contribution of internal density variations: ${ }^{160}$

$$
F_{\text {blob }}(\bar{q})=\frac{1}{\mu \bar{q} \xi} \cdot \frac{\sin (\mu \arctan (\bar{q} \xi))}{\left(1+(\bar{q} \xi)^{2}\right)^{\mu / 2}} .
$$

According to the discussion of dendrimer properties in chapter 6.1, the dendrimer density profile is modeled by a convolution of a homogeneous sphere with radius $R$ and a Gaussian distribution with standard deviation $\sigma$ (Figure 6-4). Using the fact that the Fourier transformation splits a convolution into the product of the Fourier transform of the multipliers, the scattering amplitude $A_{\text {shape }}(q)$ can be expressed as follows: ${ }^{174}$ 
6. Dendrimers

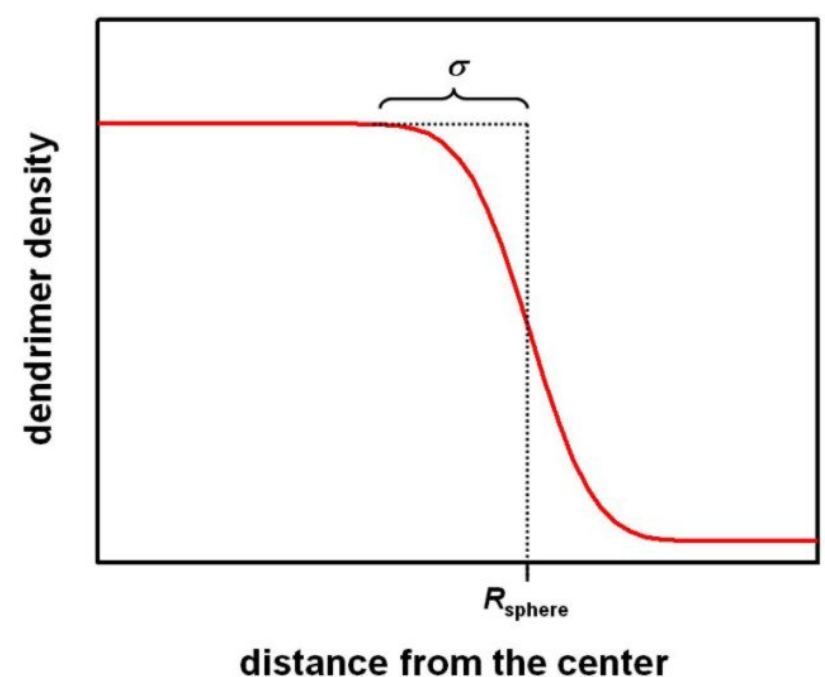

Figure 6-4: Modeled dendrimer density profile.

$A_{\text {shape }}(q)=\int_{0}^{\infty} \rho(r) r^{2} \frac{\sin (q r)}{q r} d r=\frac{3}{(q R)^{3}}[\sin (q R)-q R \cdot \cos (q R)] \exp \left(-\frac{q^{2} \sigma^{2}}{4}\right)$.

$\rho$ is the dendrimer segment density profile. The form factor can be calculated from the scattering amplitude according to the relation

$$
F_{\text {shape }}(q)=A_{\text {shape }}(q)^{2}=\frac{9}{(q R)^{6}}[\sin (q R)-q R \cdot \cos (q R)]^{2} \exp \left(-\frac{q^{2} \sigma^{2}}{2}\right) .
$$

In summery, the model for fitting the scattering intensity of dendrimer solutions described above includes four adjustable parameters: the sphere radius $R$, the width of the smeared surface region determined by the standard deviation $\sigma$ of the assumed Gaussian distribution, the correlation length $\xi$ of density variations, and the relative weighting factor $a_{\text {rel }}$. Analog models accounting for the contribution of the loose, polymeric character as well as the overall compact shape have been already successfully applied to dense polymer systems such as star polymers, ${ }^{170,175,176}$ diblockcopolymer micelles, ${ }^{177}$ and dendrimers. ${ }^{160,174,178}$ 


\subsection{Generation dependence of the dendrimer conformation}

Small angle X-ray scattering data of all dendrimers are obtained at low polymer concentrations $(\approx 2 \mathrm{wt} \%)$ at $\mathrm{pH}=8.5$. Since further dilution had no effect on the scattering intensity profile, the shown scattering data represent dendrimer form factors $F(q)$. Any influence of inter-particle interaction can be considered to be negligible at those concentrations.

In Figure 6-5a, scattering intensities are plotted versus the dimensionless, generalized variable $q R_{\mathrm{g}}$. This allows for direct comparison of generation dependent changes in the general shape, independent of particle dimensions. The commonly used logarithmic representation ensures a very sensitive visualization over a broad $q R_{\mathrm{g}}$ range. Successive data sets are separated by a multiplicative factor of 10 for the sake of clarity. Recorded scattering intensities are fitted by the fuzzy surface model developed above. Results are listed in Table 6.2 .

The form factor $F(q)$ shows the development of oscillations for high $q$ values with increasing generation number. For PAMAM dendrimers generation 6 and 8 secondary and ternary (only $G=8$ ) maxima are clearly visible. As an example, the two different contributions $F_{\text {blob }}$ and $F_{\text {shape }}$ to the overall scattering are separately plotted for PAMAM generation 8 dendrimers in Figure 6-5b. The shape contribution $F_{\text {shape }}(q)$ obtained from the density profile of a fuzzy sphere is dominating the scattering at low $q$ values. Furthermore, $F_{\text {shape }}(q)$ is reflecting the typical behavior expected for spherical objects with the occurrence of higher order maxima in the high $q$ region.

Leaving all four fit parameters adjustable, it is important to emphasize that the fuzzy sphere model presented above describes experimental scattering profiles in a very satisfactory way. Initially, it is important to briefly discuss mutual effects of the fit parameters used. The correlation length $\xi$ has been introduced as a fit parameter of the blob scattering term $F_{\text {blob }}(q)$ in above model, whereas the parameters $R$ and $\sigma$ are included in the contribution resulting from the overall shape $F_{\text {shape }}(q)$. The effect of $\xi$ on $R$ and $\sigma$ is negligible, since $F_{\text {blob }}(q)$ and $F_{\text {shape }}(q)$ are dominant on different length scales and therefore in different $q$ regions. However, $\xi$ and $a_{\text {rel }}$ are mainly affecting one another. The model derived above is most sensitive to distinguish between effects arising from the blob scattering and the detailed character of the surface, respectively, for data obtained from dendrimers of highest generations (PAMAM 6 and 8). Concerning in particular scattering profiles obtained from PAMAM dendrimers 

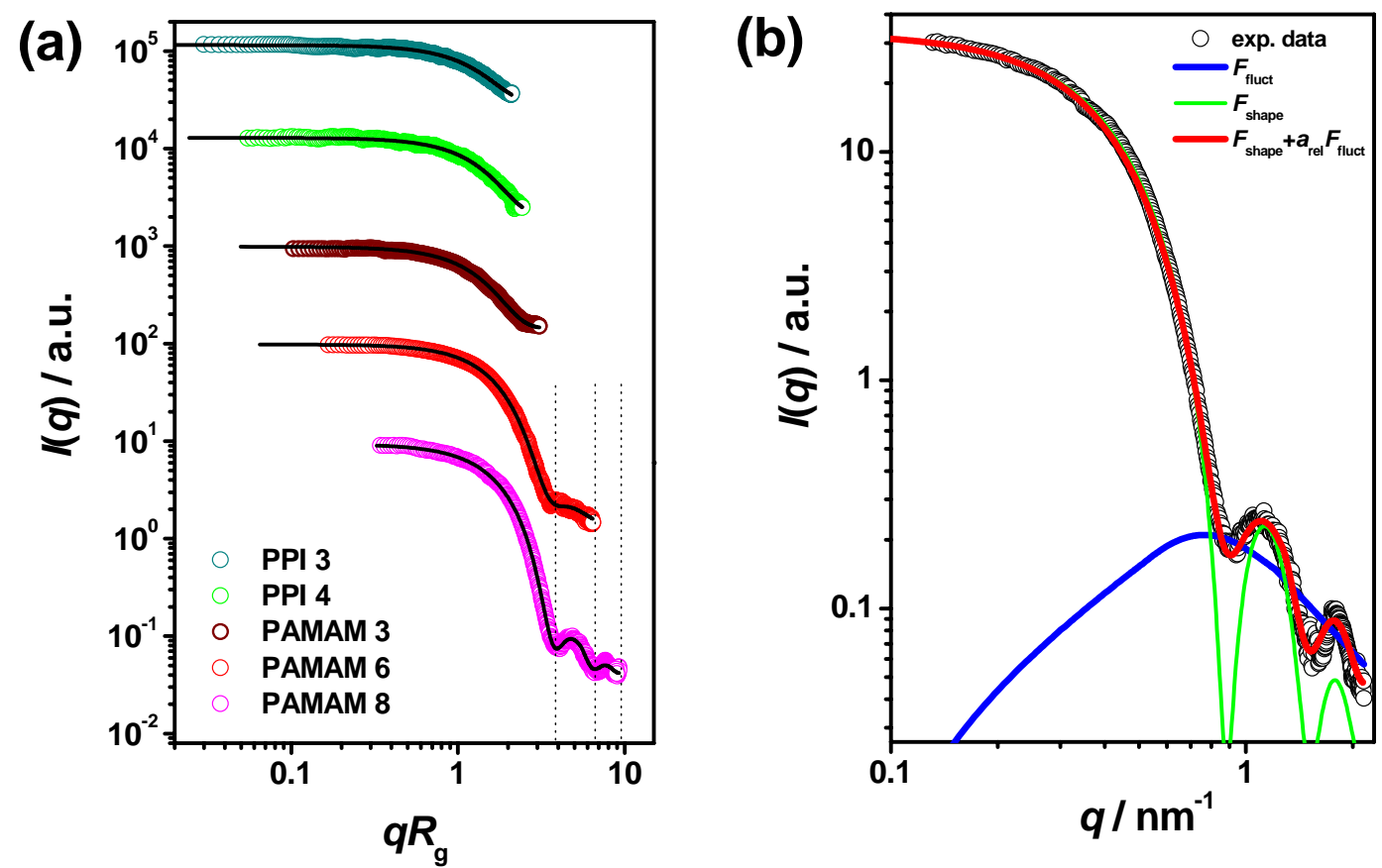

Figure 6-5: (a) Double-logarithmic plot of scattering intensities obtained from aqueous dendrimer solutions $(\mathrm{pH}=8.5)$ versus the generalized variable $q R_{\mathrm{g}}$. Data are separated by a constant factor of 10 for better visibility. Solid lines represent fits to the data using the fuzzy surface model. Positions of minimum intensity are annotated by dashed lines. (b) Comparison of modeling and experimental data for PAMAM generation 8 in double-logarithmic representation. The two contributions $F_{\text {blob }}$ and $F_{\text {shape }}$ are shown.

generation 6 and 8, the sensitivity of the fit routine can be even more increased when considering only $q$ ranges including the intermediate drop off and the second local maximum $\left(q=0.6-1.5 \mathrm{~nm}^{-1}\right)$. Separate analysis of data collected from PAMAM 6 and 8 at different $\mathrm{pH}$ conditions are initially analyzed in this $q$ region, leaving all four parameters adjustable. The obtained results show that the correlation length $\xi$ is not changing significantly or systematically around an average value of $\xi=(1.54 \pm 0.09) \mathrm{nm}$. In what follows, the correlation length is kept fixed to $1.54 \mathrm{~nm}$, thereby reducing the amount of fit parameters to three $\left(R, \sigma, a_{\mathrm{rel}}\right)$. This finding is supported by data on PAMAM dendrimers of high generations presented in literature, where the correlation length has been determined to be $\xi \approx(1.56 \pm 0.1) \mathrm{nm}$, however without considering $\mathrm{pH}^{160,178}$ 


\begin{tabular}{|c|c|c|c|c|c|}
\hline & Guinier & \multicolumn{4}{c|}{ Fuzzy sphere } \\
\cline { 2 - 6 } $\boldsymbol{G}$ & $\boldsymbol{R}_{\mathbf{g}} / \mathbf{n m}$ & $\boldsymbol{R}_{\mathbf{g}} / \mathbf{n m}$ & $\boldsymbol{R} / \mathbf{n m}$ & $\boldsymbol{\sigma} / \mathbf{n m}$ & $\boldsymbol{\sigma} / \boldsymbol{R}$ \\
\hline \multicolumn{5}{|c|}{ PAMAM dendrimers } \\
\hline 3 & $1.18 \pm 0.26$ & $1.19 \pm 0.10$ & $1.53 \pm 0.13$ & $0.79 \pm 0.08$ & 0.52 \\
\hline 6 & --- & $2.35 \pm 0.05$ & $3.04 \pm 0.07$ & $1.29 \pm 0.13$ & 0,43 \\
\hline 8 & --- & $3.88 \pm 0.02$ & $5.01 \pm 0.03$ & $1.44 \pm 0.11$ & 0.29 \\
\hline \multicolumn{7}{|c|}{ PPI dendrimers } \\
\hline 3 & $0.88 \pm 0.20$ & $0.84 \pm 0.12$ & $1.09 \pm 0.15$ & $0.57 \pm 0.05$ & 0.53 \\
\hline 4 & $1.12 \pm 0.22$ & $1.08 \pm 0.10$ & $1.40 \pm 0.13$ & $0.70 \pm 0.06$ & 0.5 \\
\hline
\end{tabular}

Table 6-2: Summary of the results obtained from fitting the SAXS data $(\mathrm{pH}=8.5)$.

The minima in intensity at $q_{\mathrm{min}} R_{\mathrm{g}}$, as shown in Figure 6-5, are most sensitive to the size of the particles. For spheres, values of

$$
q_{\min } R_{g}=\sqrt{3 / 5}(2 n+1) \cdot \pi / 2=3.65,6.08,8.52, \ldots ; \quad n \in \aleph
$$

are expected (Mie scattering). ${ }^{160}$ Measured values of $q_{\min } R_{\mathrm{g}}=3.62,6.09,8.52$ are in very good agreement with the expected values. The fact that the minima are more pronounced with increasing generation number indicates a tendency of the molecules to be become more compact and spherical having sharper boundaries. ${ }^{159,160,179}$ For smaller dendrimers, the radii of gyration $R_{\mathrm{g}}$ are additionally obtained by Guinier fits (equation (6-6)) of the low $q$ region (Table 6.2). Interestingly, the ratio of the radius of gyration and the particle radius, $R_{\mathrm{g}} / R$, varies between 0.772 (PAMAM 3 ) and 0.81 (PPI 4). This is close to the theoretical value of $\sqrt{5 / 3} \approx 0.775$ expected for an ideal, homogenous sphere.

According to equation (6-2), the molecular weight $M_{\mathrm{W}}$ of a dendrimer is proportional to $2^{G+3}-3$ for PAMAM dendrimers and $2^{G+2}-3$ for PPI dendrimers. Assuming a constant density in the core region $r \leq R, R$ is expected to obey to the following relation since the volume is proportional to the molecular weight:

$$
\begin{aligned}
R_{P A M A M} & \sim\left(2^{G+3}-3\right)^{1 / 3} \\
R_{P P I} & \sim\left(2^{G+2}-3\right)^{1 / 3} .
\end{aligned}
$$

Fitting experimental data, $R$ obtained for both types of dendrimers follows above dependence very well (Figure 6-6a), independent of the chemical character of dendrimers. The relation $R \sim M_{W}^{1 / 3}$ is indicative of a compact (space-filling) dendrimer structure with a fractal dimensionality of approximately 3 . This scaling exponent is in excellent agreement with experimental ${ }^{160}$ and theoretical results. ${ }^{43,156}$ 

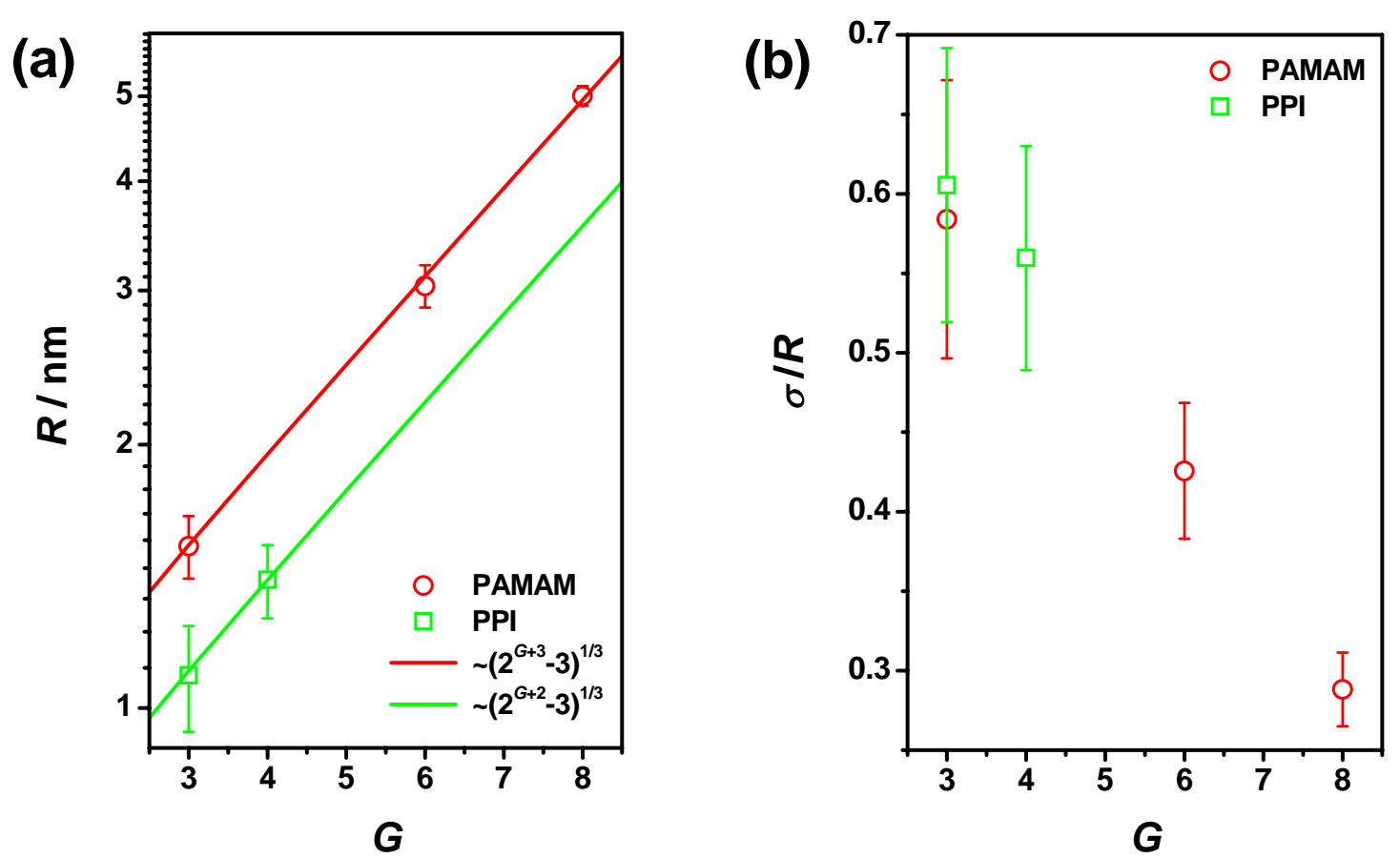

Figure 6-6: (a) Dependence of the dendrimer radius $R$ on the generation number $G$. Solid lines represent fits to the data according to equation (6-15). (b) $\sigma / R$ obtained from modeling the dendrimer surface fuzziness in dependence of the generation number $G$.

In Figure $6-6 \mathrm{~b}$, the ratio $\sigma / R$ of the width of the surface region relative to the radius of the corresponding solid sphere is plotted in dependence of the generation number. $\sigma / R$ decreases significantly with increasing $G$. Consistently, this again reflects the tendency of the molecules to extend their homogenous interior with increasing generation number.

Figure 6-5b shows that at high scattering vectors, the dendrimer form factor is significantly determined by the superposed contribution $F_{\text {blob }}(q)$ from density inhomogeneities on length scales smaller than the correlation length $\xi$. Accordingly, $F_{\text {blob }}(q)$ leads to a smearing out of higher order maxima stemming from $F_{\text {shape }}(q) .{ }^{160}$ $F_{\text {blob }}(q)$ is expected to result in a typical power-law dependence $q^{-5 / 3}$. The exponent can be derived from the Flory-Huggins parameter $v$ analogous to that obtained from a semi-dilute polymer solution under good solvent conditions.

In Figure 6-7, scattering intensities of different dendrimers are shown for high scattering vectors in a double-logarithmic representation. The limiting power-law behavior of dendrimers is still a matter of discussion in literature. From star polymers a $q^{-5 / 3}$ power-law behavior is known, ${ }^{180}$ while particles with sharp interfaces between particle and solvent exhibit $q^{-4}$ power-law behavior. ${ }^{181}$ 


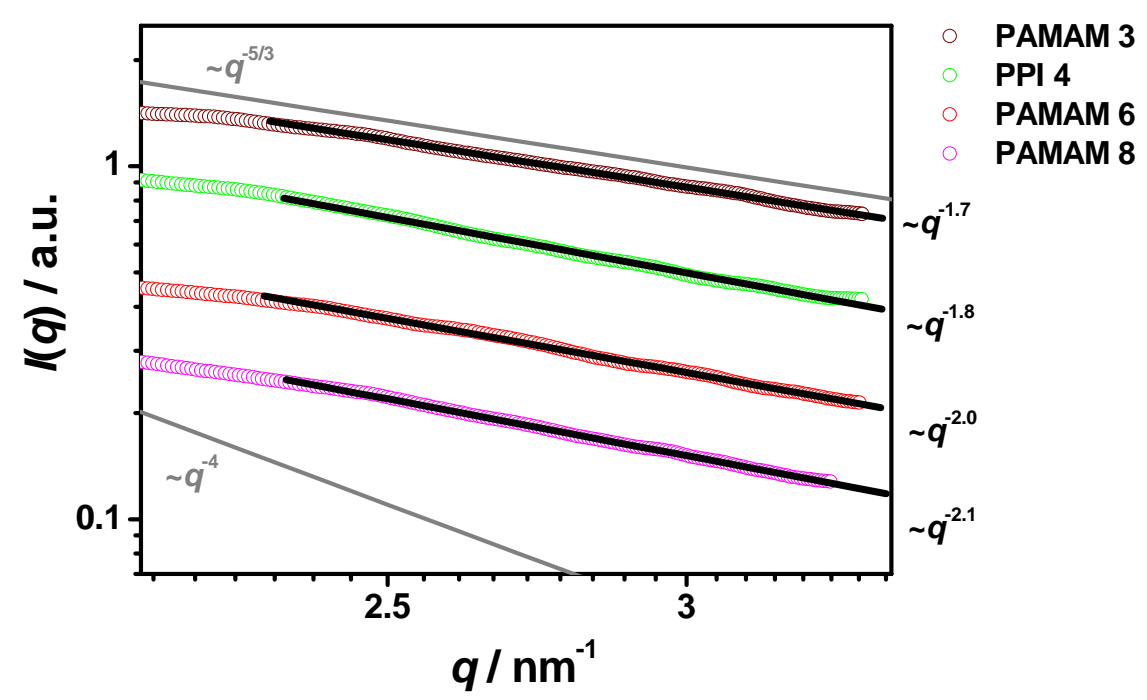

Figure 6-7: Double-logarithmic representation of the power-law dependence in the high $q$ region. Black lines represent linear fits to the experimental data. $q^{-5 / 3}$ and $q^{-4}$ power-law decays are shown for comparison as gray lines.

A transition from a $q^{-4}$ to $q^{-5 / 3}$ power-law decay with decreasing dendrimer generation for the high $q$ region, which is accompanied by a disappearance of higher order maxima, is reported from SAXS analysis of PPI and PAMAM dendrimers. ${ }^{159,} 182$ Accordingly, a transition from a rather compact, spherical shape of high-generation dendrimers to a much looser, star-like shape of low-generation dendrimers is assumed. ${ }^{159,182}$ Contrary to these findings, Rathgeber et al. observe a $q^{-5 / 3}$ power-law decay for PAMAM dendrimers independent of generation number. ${ }^{160,174}$

Experimental data shown in Figure 6-7 exhibit a slight progression in power-law behavior with generation number. This finding is valid for PPI as well as PAMAM dendrimers. Exponent values range from -1.7 for dendrimers of generation 3 to -2.1 for dendrimers of generation 8 . Observed changes in limiting power-law exponent with generation number are small compared to SAXS results presented in reference ${ }^{159}$ and ${ }^{182}$. Supported by the appearance of oscillation in the high $q$ region with increasing generation number, which are typical for spherical objects, these experimental findings could be interpreted as resulting from a conformational transition towards a more compact shape as this has been done in references ${ }^{159}$ and ${ }^{182}$. However, such a conclusion is not mandatory, as is demonstrated in what follows.

In Figure 6-8, values obtained for the relative weighing factor of the two scattering contributions $a_{\text {rel }}$ are plotted in dependence of generation number in semi-logarithmic representation. $a_{\text {rel }}$ can be consider in analogy to considerations of Richter et al. for star polymers $^{183}$ as the ratio of intensities $a_{\text {rel }}=I(1 / \xi) / I(0)$ of the two scattering contributions. $I(1 / \xi)$ is the scattering intensity on length scale $q \approx 1 / \xi$ of the blob size. 


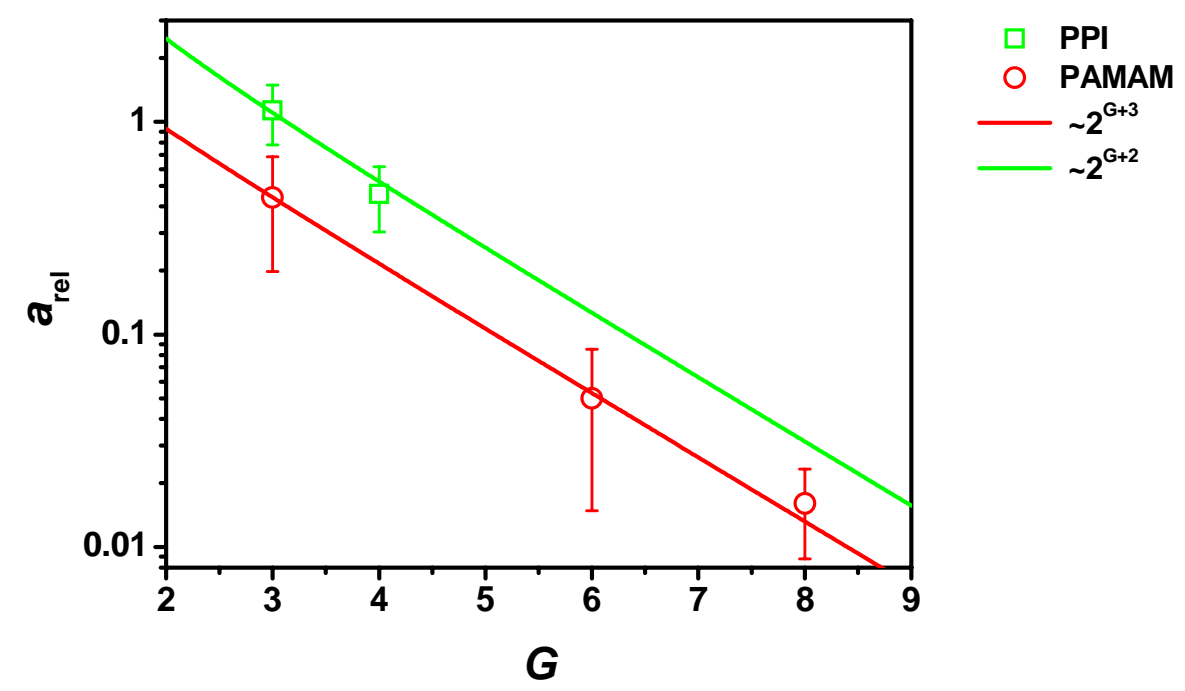

Figure 6-8: Relative weighing factor $a_{\text {rel }}$ of the two scattering contributions as a function of the generation number, $G$. Solid lines represent fits to the data according to equation (6-16).

At these high $q$ values, an incoherent superposition of the coherent scattering from within each blob occurs. Accordingly, the scattering intensity can be expressed as $I(1 / \xi) \sim N_{\text {blob }} N_{\text {seg }}^{2} . N_{\text {blob }}$ is the number of blobs and $N_{\text {seg }}$ is the number of segments per blob. In the Guinier regime at $q=0$, scattering contributions of all segments add up coherently and the scattering intensity of the whole molecule becomes proportional to $I(0) \sim\left(N_{\text {blob }} N_{\text {seg }}\right)^{2}=N_{\text {tot }}^{2}$ with $N_{\text {tot }}$ being the total number of segments in the whole dendrimer. $a_{\text {rel }}$ is therefore given by

$$
\begin{aligned}
a_{r e l} & =\frac{N_{b l o b} N_{\text {seg }}^{2}}{N_{\text {tot }}^{2}}=\frac{N_{\text {seg }}}{N_{\text {tot }}} \\
a_{r e l}^{\text {PAMAM }} & =N_{\text {seg }}^{\text {PAMAM }}\left(2^{G+3}-3\right)^{-1}, \\
a_{r e l}^{P P I} & =N_{\text {seg }}^{\text {PPI }}\left(2^{G+2}-3\right)^{-1},
\end{aligned}
$$

i.e. $a_{\text {rel }}$ is the ratio of the number of scatterers in one blob to the total number of scatterers $N_{\text {tot }}$ given by equation (6-2). Since the blob size does not depend on the generation of dendrimers, it is reasonable to assume that the number of segments within a blob $N_{\text {seg }}$ is independent of generation number, too. Fits to the experimental data according to above equations show good agreement within the experimental accuracy (Figure 6-8). The number of segments within a blob is determined to be $N_{\text {seg }}^{\text {PAMAM }} \approx 27$ and $N_{\text {seg }}^{P P I} \approx 32$ for PAMAM and PPI dendrimers, respectively. This is in good 
agreement with small angle scattering results reported in literature for PAMAM dendrimers $\left(N_{\text {seg }} \approx 22\right)$. ${ }^{160}$

It is important to notice that $a_{\text {rel }}$ increases significantly with decreasing generation number for both types of dendrimers. This indicates that the limiting power-law behavior is increasingly dominated by scattering from the internal, loose polymeric

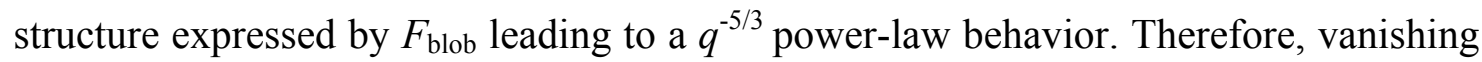
of higher-order maxima in the sphere form factor is not necessarily a consequence of changes in the overall shape of dendrimers and therefore it is not a sensitive measure for potential structural changes.

\section{4. $\mathrm{pH}$ dependence of dendrimer conformation}

Figure 6-9 shows exemplarily the absolute scattering intensity of one representative of each dendrimer type at low $\mathrm{pH}$, neutral and high $\mathrm{pH}$ conditions in a double logarithmic representation. Solid lines give results obtained from fitting the data with the fuzzy sphere model. Successive data sets are offset in intensity by a constant multiplicative factor to ensure clarity of presentation.

With decreasing $\mathrm{pH}$, the form factor $F(q)$ of PAMAM 8 dendrimers shows more pronounced oscillations for high $q$ values. Furthermore, minima in intensity at $q_{\min } R_{\mathrm{g}}$ are shifted towards higher $q$ values with increasing $\mathrm{pH}$ indicating that the size of dendrimers is changing. To quantify these changes in size, dendrimer radii $R$ are plotted in dependence of the solution $\mathrm{pH}$ in Figure 6-10 for three selected dendrimers. Resulting from intra-polymeric Coulomb interactions, a significant progression of dendrimer radius is observable with $\mathrm{pH}$ variation. To understand this behavior, the $\mathrm{pH}-$ dependent protonation mechanism of dendrimer amino groups has to be taken into account.

The microscopic charging mechanism of PAMAM and PPI dendrimers has recently been investigated in detail by potentiometric (acid-base) titrations. ${ }^{36,37,184}$ For PAMAM as well as for PPI, titration curves of dendrimers of generation 3 or higher show only minor differences. Both types of dendrimers are characterized by two distinct protonation steps and one intermediate plateau. An almost independent protonation of primary and tertiary groups has been observed. However, different protonation mechanisms are observed for the two species, which are schematically represented in Figure 6-11. 

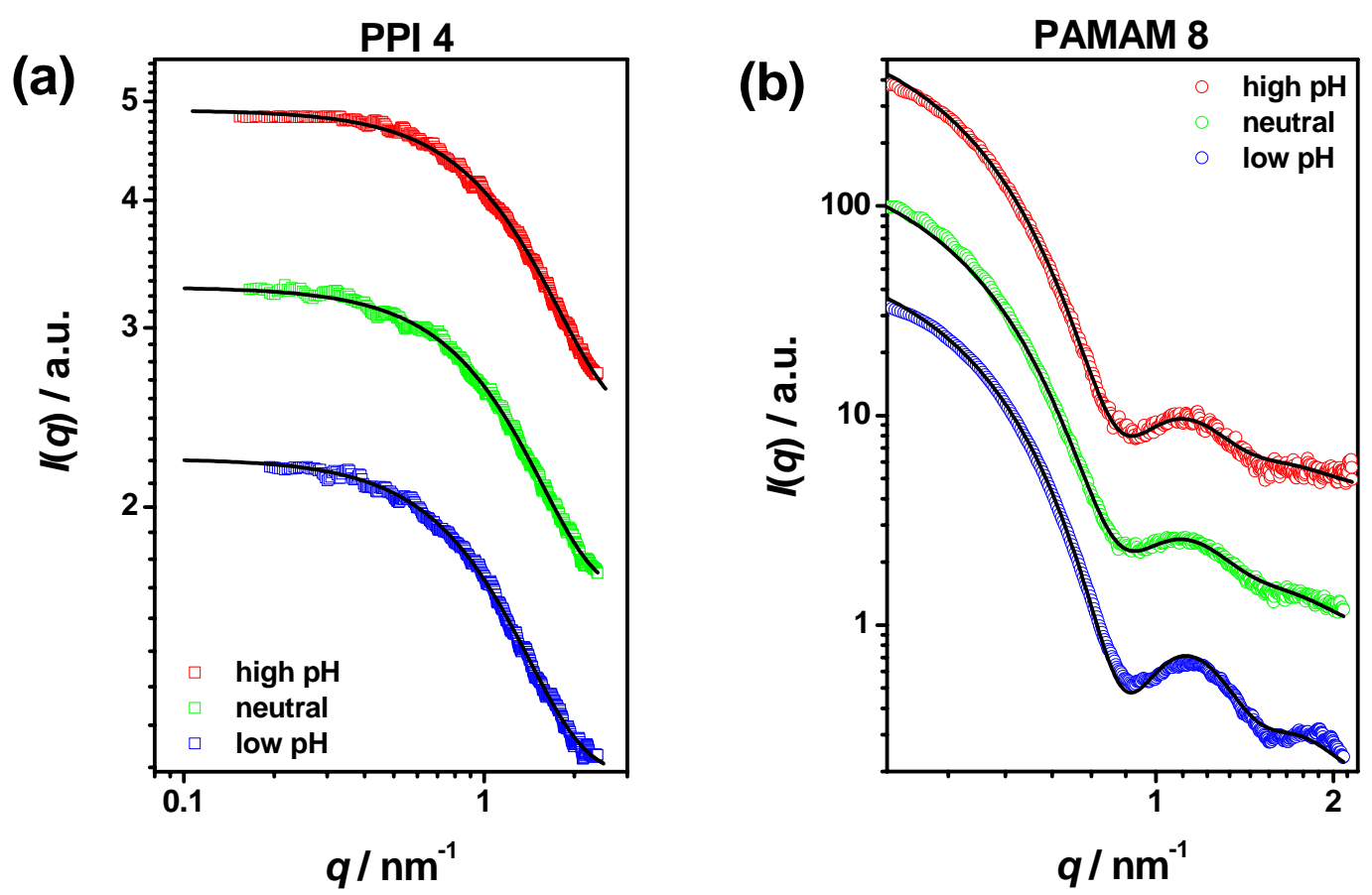

Figure 6-9: SAXS data obtained from dendrimers at low pH, neutral, and high $\mathrm{pH}$ conditions. Data for PPI generation 4 (a) and PAMAM generation 8 (b) are shown exemplarily. Solid lines are fits to the data according to the fuzzy sphere model.

Exposed to high $\mathrm{pH}$ values $(\mathrm{pH}>10)$, all amino groups of PAMAM dendrimers are unprotonated and the dendrimer are uncharged. This results in an increased tendency of the terminal units to fold back into the dendrimer interior leading to a dense-core conformation and smaller radii. In a first protonation step, primary amines of the outer layer of the PAMAM dendrimer protonate independently $\left(\mathrm{pK}_{\mathrm{I}} \approx 9.0-9.2^{36,184}\right)$. The result is a stable conformation with all primary amines protonated and all tertiary amines deprotonated. The arising electrostatic repulsion between like-charged end groups reduces back folding of dendrimer branches and leads to the increase in radius with reducing $\mathrm{pH}$ observable in Figure 6-10.

For PAMAM dendrimers, the intermediate plateau has been observed around $\mathrm{pH}=7$. In this $\mathrm{pH}$ region, changes in $\mathrm{pH}$ are not or only very slightly effecting the degree of protonation of dendrimers. This is reflected in Figure 6-10: for PAMAM 6 and 8 the radius $R$ stays almost constant between $\mathrm{pH} \approx 6.7-7.3$.

Lowering the $\mathrm{pH}$ of the solution further, PAMAM tertiary amino groups protonate $\left(\mathrm{pK}_{\text {III }} \approx 5.8^{36}\right)$. This process leads to a stable state, where all amino groups are protonated, with the exception of one central tertiary amino group. As a consequence of increased resulting intra-polymeric Coulomb repulsions, dendrimer branches are further 
6. Dendrimers
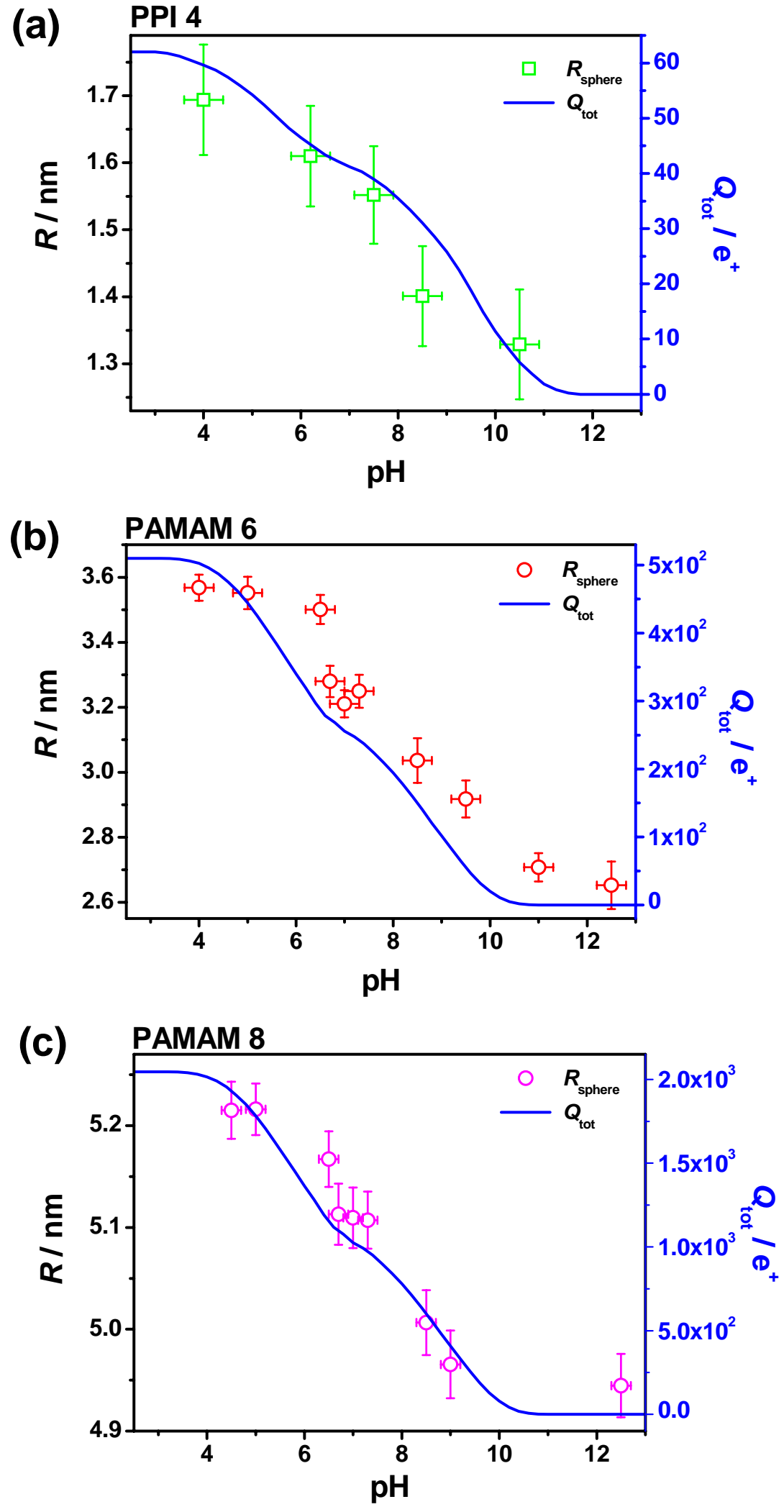

Figure 6-10: Dependence of the dendrimer radius $R$ (open circles) on the pH value of the solution for (a) PPI 4, (b) PAMAM 6, and (c) PAMAM 8 dendrimers. Solid lines give changes of the total dendrimer charge $Q_{\text {tot }}$ resulting from varying the $\mathrm{pH}$. 


\section{Dendrimers}

(a) PAMAM

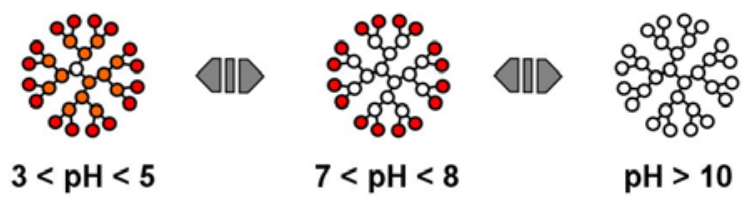

(b) PPI

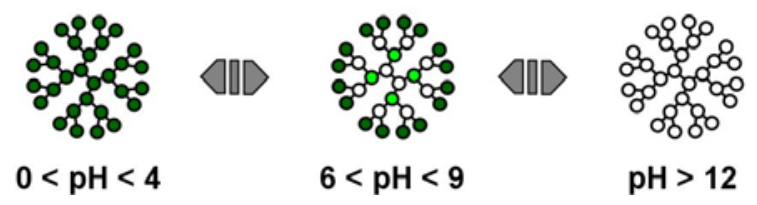

Figure 6-11: Schematic representation of the microscopic protonation mechanism for PAMAM (a) and PPI dendrimers (b) of generation 3 and higher.

extended. Figure $6-10$ shows that for PAMAM generation 6 and 8 starting at $\mathrm{pH} \approx 6$ no further increase in the dendrimer radius is observed with reducing $\mathrm{pH}$, although the degree of protonation is still increasing. This indicates that PAMAM 6 and 8 have already reached a conformation, which is characterized by a maximum degree of stretching of polymeric subunits. In addition, with increasing $\mathrm{pH}$ screening effects could potentially effect the behavior of polymeric segments. ${ }^{43}$ Owing most likely to their less densely packed conformation, dendrimers of lower generations do not exhibit a comparable saturation in radius at low $\mathrm{pH}$ conditions. The last group to protonate is one of the central tertiary amine groups $\left(\mathrm{pK}_{\mathrm{IIIb}} \approx 3.5^{36}\right)$. For large dendrimers, however, this last protonation step contributes very little to the overall charge.

For PPI dendrimers, the intermediate plateau lies at a degree of protonation of $2 / 3$ of the total ionizable groups. During the first protonation step occurring around $\mathrm{pH} \approx 10$, PPI primary amine groups and the odd shells of PPI tertiary amine groups protonate. ${ }^{37}$ Again, arising electrostatic intra-molecular repulsion is reflected by the observable increase in radius (Figure 6-10a). The relatively unpronounced intermediate plateau around $\mathrm{pH} \approx 7$ results from the stability of this onion-like structure where all odd shells of the dendrimer are protonated, while the even ones remain deprotonated (Figure 6-11). This protonation pattern reflects short-ranged repulsive interactions between ionizable sites. In the second step around $\mathrm{pH} \approx 5$, PPI tertiary amine groups of even shells protonate. ${ }^{37}$

Differences in protonation mechanisms between PPI and PAMAM dendrimers are understandable by considering that PAMAM dendrimers exhibit weaker nearest neighbor interactions owing to the fact that the distance between two neighboring binding sites is much larger than it is for PPI dendrimers (Figure 6-2). In addition, ion pairing further diminishes these nearest neighbor interactions. ${ }^{184}$ 


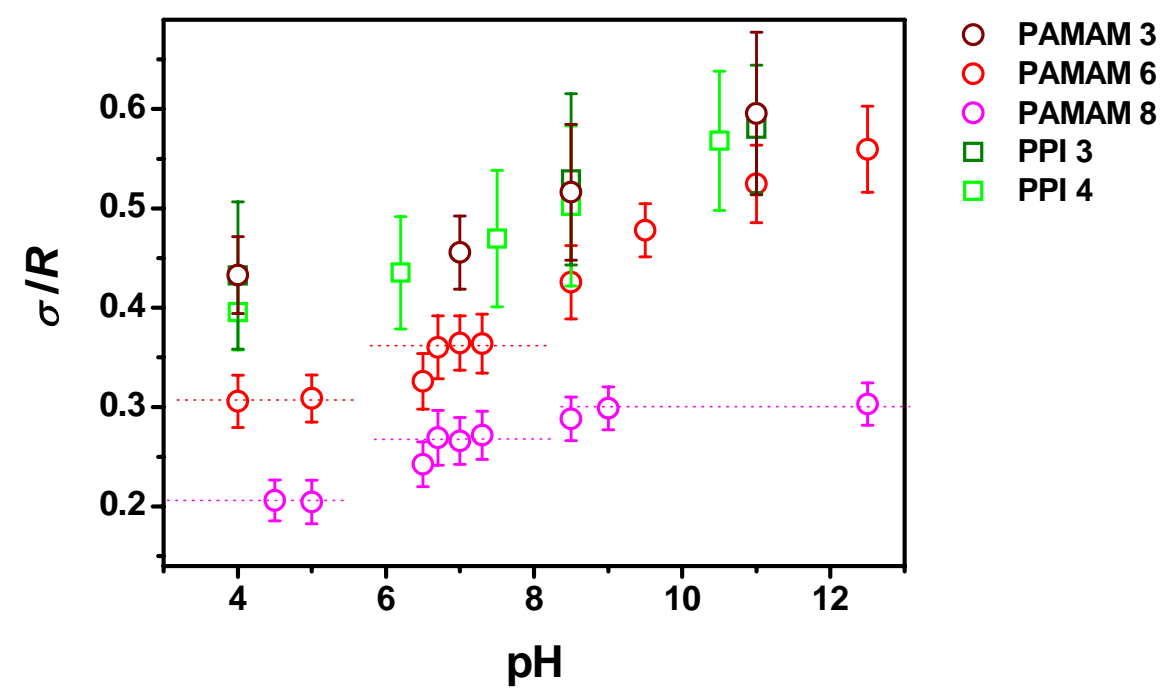

Figure 6-12: Dependence of $\sigma / R$ of different types of dendrimers on pH. Dashed lines are guides to the eye only.

In Figure 6-12, the ratio $\sigma / R$, which reflects the proportion of the width of the fuzzy surface region relative to the dendrimer interior with homogenous density, is plotted in dependence of $\mathrm{pH}$ conditions. For all degrees of protonation, $\sigma / R$ is increasing with decreasing generation number, as has already been shown in Figure 6-6b for $\mathrm{pH}=8.5$. Moreover, independent of the dendrimer type and the generation, $\sigma / R$ increases with increasing $\mathrm{pH}$. These findings indicate a tendency of the molecules to become more compact and spherical having sharper boundaries with increasing generation number and/or charge. With increasing electrostatic repulsion, dendrimer segments tend to increasingly stretch, and dendrimers increasingly behave as hard spheres. According to changes in radii, for PAMAM $8 \sigma / R$ exhibits only small changes throughout all degrees of protonation. This insensitivity could again be a result of the high generation number, which indeed leaves no room for additional back folding or stretching of polymer branches due to very high steric crowding. Furthermore, for PAMAM 6 and $8 \sigma / R$ stays constant for $\mathrm{pH}$ values close to the intermediate plateau region of corresponding titration curves, where changes in the degree of protonation are small.

Both, the dendrimer size and the charge, are of crucial importance for the compaction of DNA by dendrimers. With results from potentiometric titration experiments ${ }^{36,37,184}$ at hands, it is possible to translate changes in $\mathrm{pH}$ into variations of the total molecular charge $Q_{\text {tot }}$. Accordingly, Figure 6-13(a-c) shows the charge dependence of dendrimer radii for the three dendrimers presented in Figure 6-10, whereas Figure 6-13(d) gives the ratio $\sigma / R$ (Figure 6-12) in dependence of the normalized dendrimer charge 

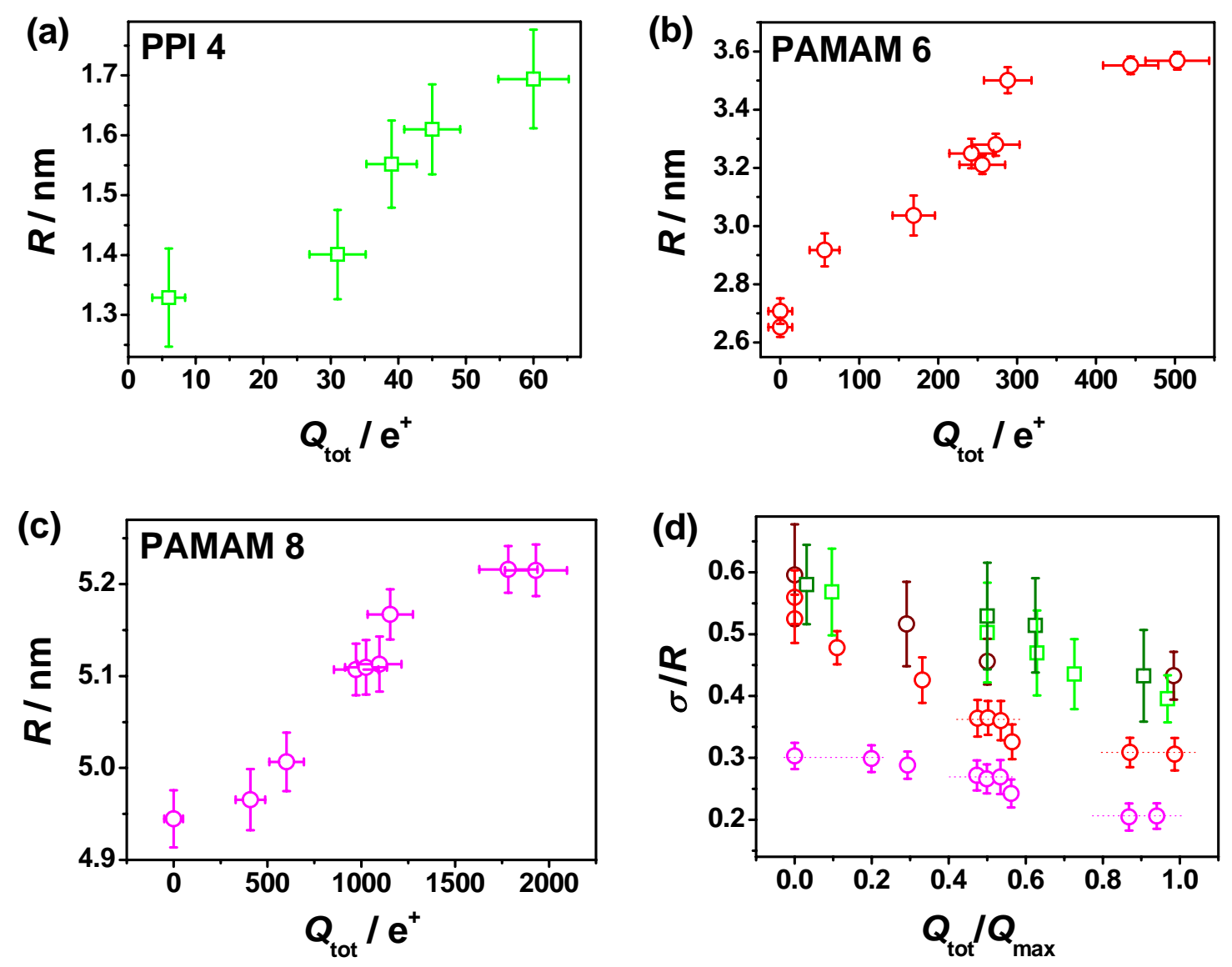

Figure 6-13: Dependence of the dendrimer radius $R$ on the total molecular charge for (a) PPI 4, (b) PAMAM 6, and (c) PAMAM 8 dendrimers. (d) Dependence of $\sigma / R$ on the normalized charge $Q_{\text {tot }} / Q_{\max }$.

$Q_{\text {tot }} / Q_{\text {max }}$. At $Q_{\text {tot }}=0$, dendrimer radii are minimal due to the fact that the dendrimer branches are folded back to the interior. Consistently, $\sigma / R$ adopts maximum values at $Q_{\text {tot }} / Q_{\max }=0$. Continuous charging of the dendrimer initially results in a successive increase of $R$ and a decrease of $\sigma / R$. However, both quantities level off after approximately $65-70 \%$ of all amino groups are protonated. The fact that a protonation of the last $25-30 \%$ of all tertiary amino groups has no effect on both $R$ and $\sigma / R$ for all studied dendrimers is corresponding to observations for $\mathrm{pH}<6$.

In order to determine the $\mathrm{pH}$ dependence of dendrimer conformation, dendrimer volumes $V$ are normalized by corresponding volumes of uncharged dendrimers at high $\mathrm{pH}$ conditions, $V_{0}$, and plotted versus $\mathrm{pH}$ in Figure 6-14. $V / V_{0}$ is continuously increasing with decreasing $\mathrm{pH}$ and therefore increasing molecular charge. Generally, with increasing generation number, the increase in volume is more pronounced. For PAMAM 8 however, significant deviations from this behavior are observable 


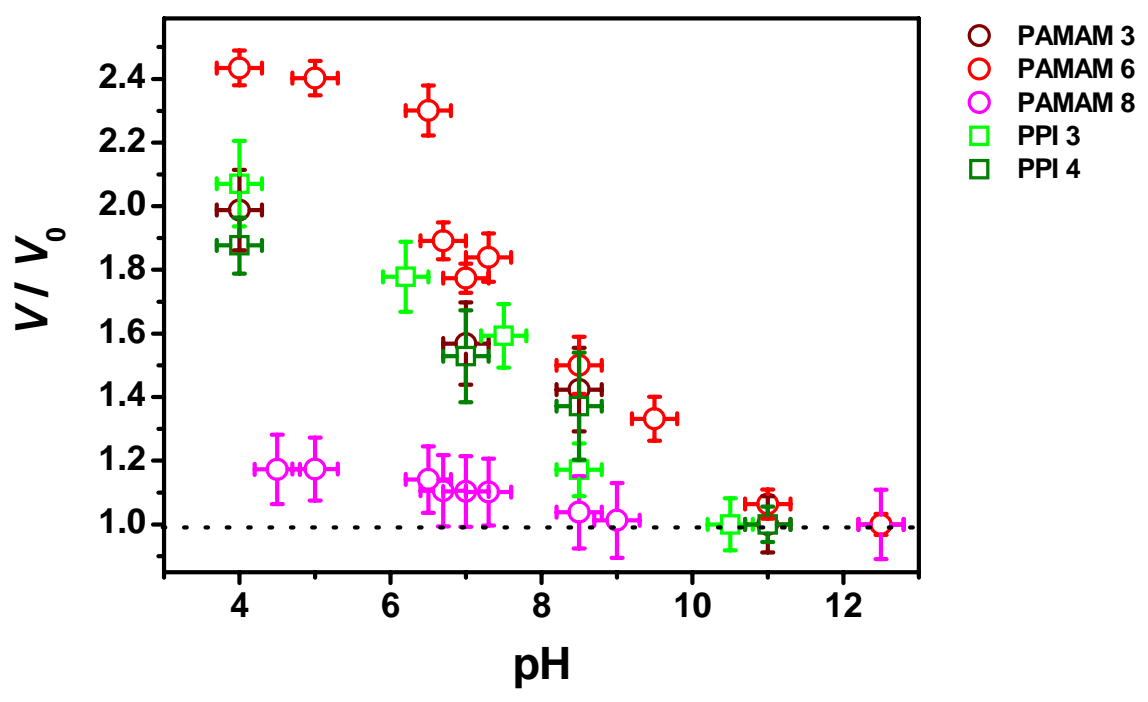

Figure 6-14: pH dependence of dendrimer volumes normalized with respect to their values at $\mathbf{p H}=12$.

exhibiting only comparably small changes throughout all degrees of protonation. This reflects the significantly higher average density - in particular of the outer segment layers - of high generation dendrimers. To account for the exponentially growing volume fraction needed for successive generations, interior polymer segments of lower generation numbers must already be significantly stretched.

Charge-induced changes in radius and volume relative to values at high $\mathrm{pH}$ conditions are given in Figure 6-15. In general, changes between high $\mathrm{pH}$ and neutral conditions are larger than between neutral and low $\mathrm{pH}$ conditions for all dendrimers. This finding reflects the fact that $\approx 50 \%$ of all dendrimer segments are part of the outer layer and therefore possess primary amino (end) groups, which are protonated at neutral conditions. Furthermore, screening effects could play a role at low $\mathrm{pH}$ values. ${ }^{43}$ Expressed in terms of dendrimer volume, dendrimers exhibit changes of up to approximately $150 \%$ (PAMAM 6) when the $\mathrm{pH}$ is varied over the whole range! In particular regarding their application, it is important to notice that the sequence of conformational changes is reversible for dendrimers of all types and generations. Owing to its reproducibility, this was termed a "smart behavior". 39

The only experimental study so far on charge-induced dendrimer swelling reported no changes in size of PAMAM 8 upon variation of $\mathrm{pH}$ or ionic strength. ${ }^{38}$ Figure 6-14 shows that conformational changes of PAMAM 8 are indeed very small and - in the scope of error bars - could indeed be zero. However, this is a particular phenomenon resulting from the fact that PAMAM 8 already represents the high molecular limit. 

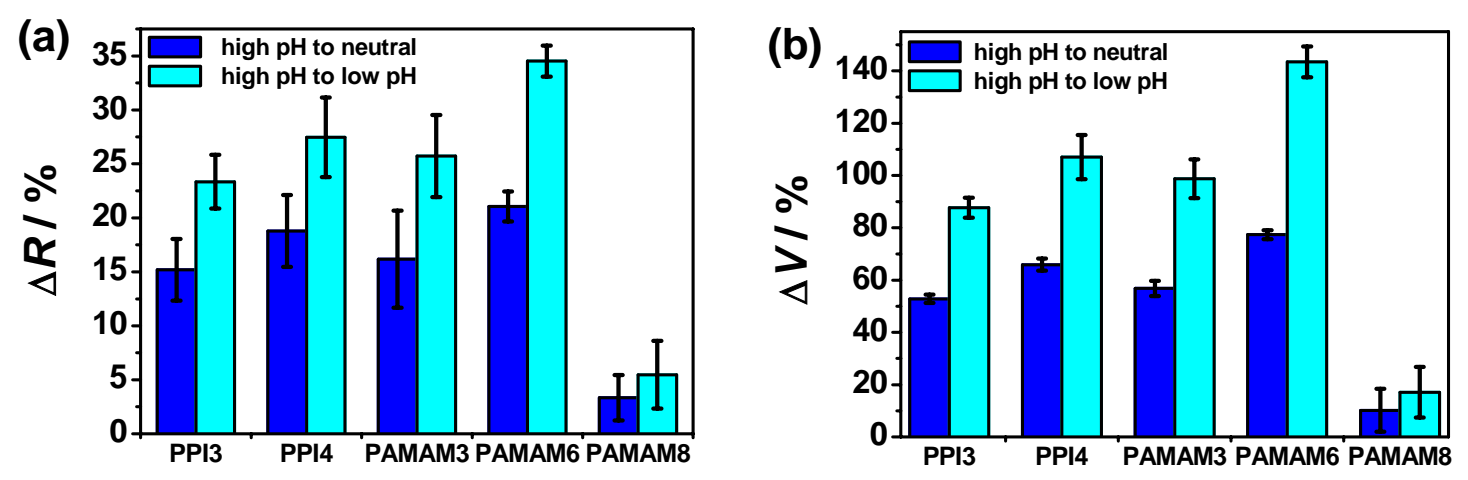

Figure 6-15: $\mathrm{pH}$ induced proportional changes in dendrimer radii (a) and volumes (b).

These results clearly show charge-induced changes of the dendrimer conformation eliminating the discrepancy between theory and experiments that existed in the literature up to now.

Comparing the experimental results to simulations reported in the literature, qualitatively they are in best agreement with results obtained from MD simulations of the conformation of PAMAM dendrimers generation 2-6 at different $\mathrm{pH}$ conditions. ${ }^{40}$ The authors report a conformational change of dendrimers from compact objects at high $\mathrm{pH}$ values to open ones at low $\mathrm{pH}$ values. However, simulations were performed without explicitly accounting for water molecules, but rather using the assumption of a continuous solvent inherent in a single dielectric screening constant $\varepsilon(r)$ and a linearscreening Debye-Hückel theory for the counter-ion interaction. Both assumptions are bound to break down at short intra-molecular distances between charged dendrimer units. Therefore, it is not surprising that the magnitudes of $\mathrm{pH}$ induced conformational changes of dendrimers obtained from simulations are in parts much higher than in the experimental results.

From the data presented above, information can be deduced about the density of dendrimers and about the amount of solvent in the interior of dendrimer molecules. The smaller the radius (and therefore the volume), the smaller is the solvent content inside the dendrimeric structure, and vice versa.

At high $\mathrm{pH}$ values, dendrimers adopt conformations with polymeric segments folded back exhibiting minimal radii and volumes. Knowing dendrimer radii and molecular weights (Table 5-1), it is possible to determine dendrimer densities $\rho$. For PAMAM 3, 6 and 8 , values of $\rho=1.09 \mathrm{~g} \mathrm{~cm}^{-3}, 1.23 \mathrm{~g} \mathrm{~cm}^{-3}$, and $1.23 \mathrm{~g} \mathrm{~cm}^{-3}$, respectively, are obtained, whereas PPI 3 and 4 exhibit smaller densities of $\rho=0.74 \mathrm{~g} \mathrm{~cm}^{-3}$ and $0.73 \mathrm{~g}^{\mathrm{cm}}{ }^{-3}$, respectively. 


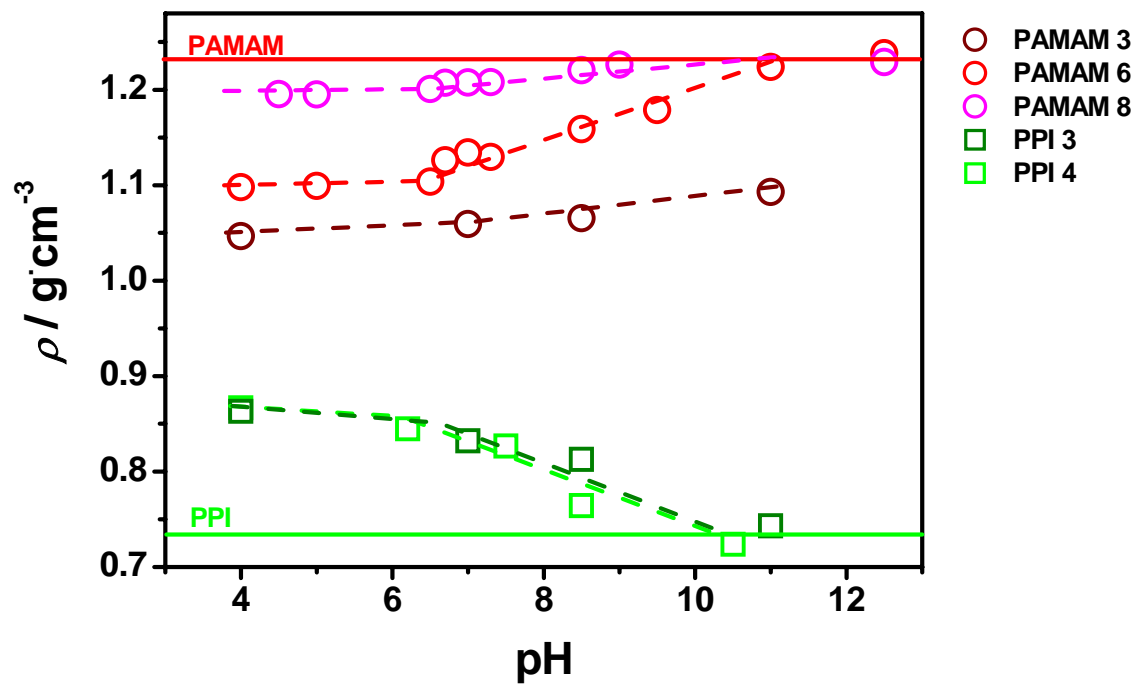

Figure 6-16: $\mathrm{pH}$ dependence of dendrimer density. Solid lines indicate the bulk densities, dashed lines are guides to the eye only.

In literature, bulk densities ranging from $\rho_{\text {lit }}=1.214-1.224 \mathrm{~g}^{\cdot} \mathrm{cm}^{-3}$ for PAMAM generations 3-5 are reported. ${ }^{185}$ Furthermore, based on experimental data of lower PAMAM generations, dendrimer densities of generation 6-9 have been calculated resulting in monotonically increasing densities with a maximum value of $\rho_{\text {lit }}=1.232 \mathrm{gcm}^{-3}$ for PAMAM 9. ${ }^{186}$ Compared to these values, experimental results show a good agreement for PAMAM 6 and 8. However, the determined density of PAMAM 3 deviates significantly from its bulk value.

Since there are no values for PPI dendrimer density reported in literature, it is necessary to compare obtained results to reported densities of condensed organic compounds, which have a chemical composition similar to PPI segments (e.g. $\left(\mathrm{C}_{3} \mathrm{H}_{7}\right)_{2} \mathrm{NH}$, $\left.\left(\mathrm{C}_{2} \mathrm{H}_{5}\right)_{2} \mathrm{CHNH}_{2}, \mathrm{C}_{4} \mathrm{H}_{9} \mathrm{NH}_{2}\right)$. Densities of these compounds are in the range of $\rho_{\text {lit }} \approx 0.73-0.75 \mathrm{~g} \mathrm{~cm}^{-3}$ yielding a good agreement with obtained results. ${ }^{187}$

The $\mathrm{pH}$-dependence of dendrimer densities is given in Figure 6-16. With a decreasing $\mathrm{pH}$, dendrimer radii and volumes increase. This yields an additional interior space, which makes dendrimers well suited for host-guest interactions and encapsulations of guest molecules. Dendrimers being solved in water, the additional interior space $\Delta V$ is occupied by an increasing amount of water molecules (molecular volume $\left.V_{\text {water }}=30.1 \AA^{3}\right)$. ${ }^{188}$ Accordingly, reducing the $\mathrm{pH}$ dendrimer densities approach the density of the incorporated water molecules, $\rho=1 \mathrm{~g} \mathrm{~cm}^{-3}$.

Figure 6-16 shows that for $\mathrm{pH} \geq 10$ only the PAMAM 3 density does not approach its expected bulk value. This indicates that even completely uncharged PAMAM 3 dendrimers with completely back-folded branches still contain a certain amount of 


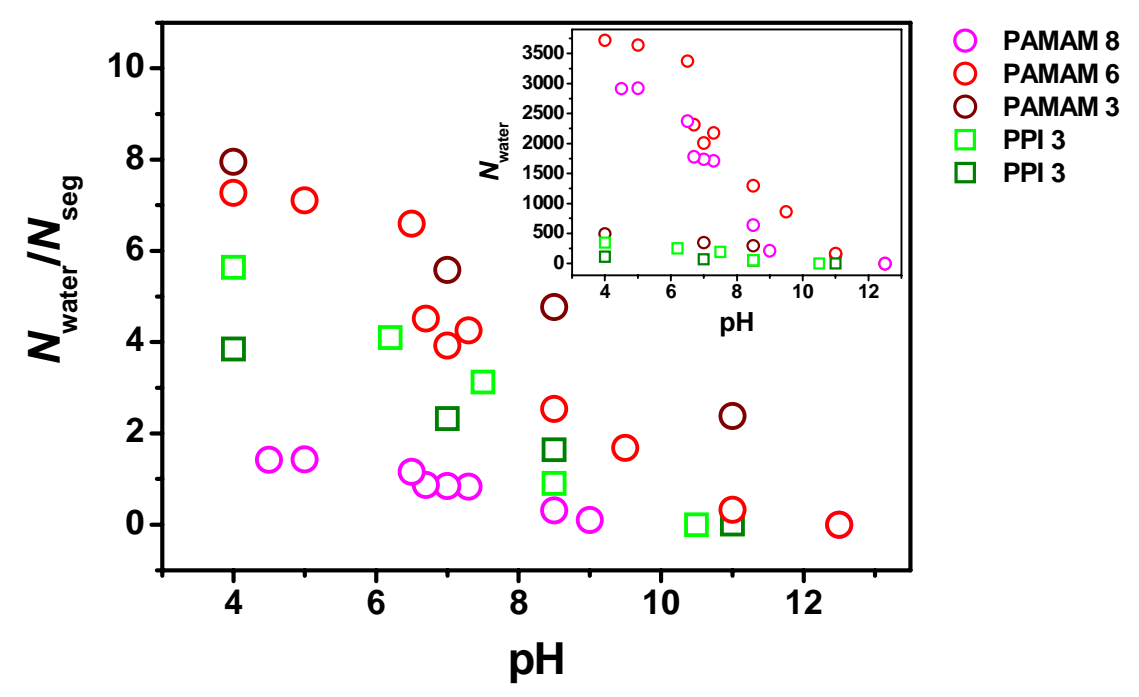

Figure 6-17: Amount of water molecules inside the dendrimers at different pH.

water molecules in their interior. This is contrary to observations for the similar-sized PPI 4 and the smaller PPI 3 dendrimers. An explanation for this behavior is provided by the nature of PAMAM dendrimer branches: compared to PPI branches they are significantly longer and, in addition, they contain hydrophilic carboxyl groups.

In Figure 6-17, the pH-dependent increase of the amount of water molecules per polymer segment $N_{\text {water }} / N_{\text {seg }}$ inside dendrimers is given, whereas the absolute amount of water molecules is given in the inset. Highest amounts of water molecules are found for PAMAM generation 3 and 6. PAMAM 6 yields a maximum amount of about 7 water molecules per segment, whereas the open, undeveloped dendrimer structure of PAMAM 3 with its comparatively long branches enables 8 water molecules per segment.

Besides changing solution $\mathrm{pH}$, conformational changes of dendrimers can be achieved by addition of salt. Increasing the salt concentration leads to an increase in the Debye screening parameter $\kappa$. Consequently, at high ionic strengths, the Coulomb repulsion between the charged dendrimer branches is so strongly screened that dendrimers are expected to behave similar to uncharged ones.

The huge variety of exciting potential applications behind this "smart behavior" of dendrimers can easily been envisioned. Two of the most important, underlying principles are denoted briefly: 


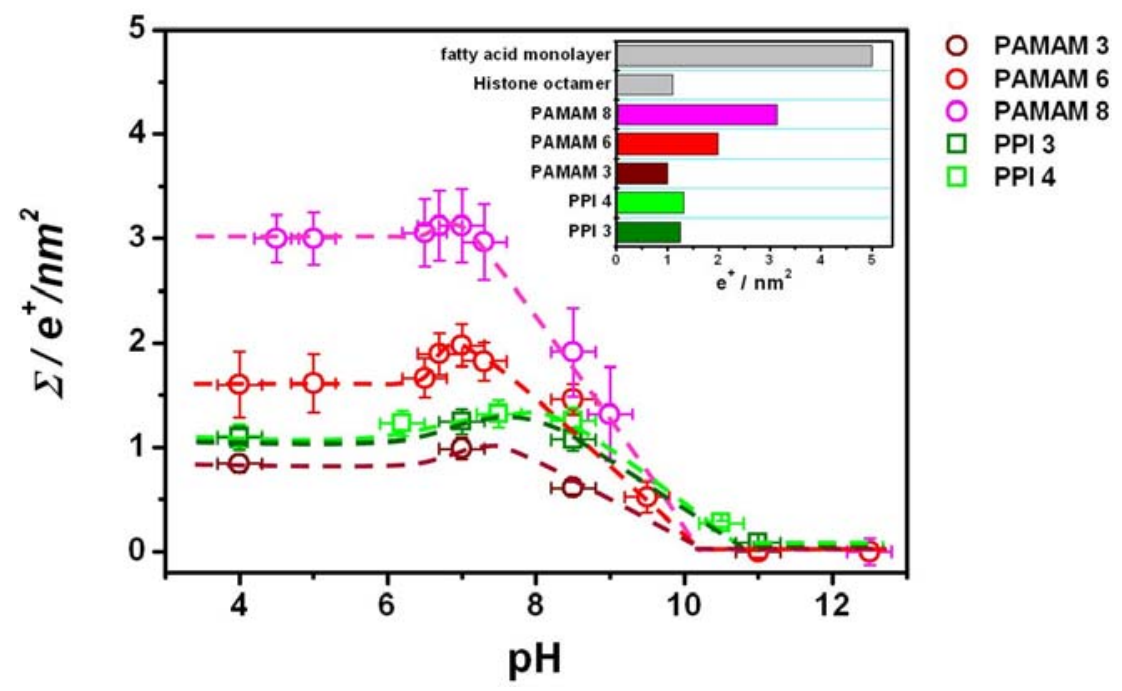

Figure 6-18: pH-dependence of the surface charge density. Dashed lines are guides to the eye only.

- The free volume in the interior of highly charged dendrimers can be used to allow for introduction of smaller molecules (i.e. drugs) into the hollow host. Reducing the effective charge, e.g. by exposure to a highly salted environment such as human blood, would force the dendrimers to collapse back into their dense conformation releasing thereby guest molecules in their interior into the living organism.

- There are several paths of uptake of exogenous materials into a cell with endocytosis being the most efficient way for macromolecules. Consequently, escape from the endosome is critical for the cytosolic delivery of any macromolecule before it is degraded in the lysosome (local $\mathrm{pH} \approx 5$ ). One of the main reasons for the high transfection efficiency obtained with dendrimers is due to both polymer expansion and osmotic induced swelling from protonation of tertiary amines, which has been observed to cause endosomal rupture and release of the complex or DNA into the cytoplasm. ${ }^{40,189-191}$

Therefore, possible conformational changes of dendrimers upon charging and the possibility of controlling it externally through changing the $\mathrm{pH}$ and/or the ionic strength of the solution are most certainly very promising areas of research.

In the context of a dendrimer induced DNA compaction, the surface charge density $\Sigma$ of the compaction agent is of special importance:

$$
\Sigma=Q_{\mathrm{I}} / O
$$

with $Q_{\mathrm{I}}$ being the charge of the primary amine (end-)groups and $O=4 \pi R^{2}$ being the dendrimer surface area. Protonated tertiary amine groups situated well in the dendrimer 


\section{Dendrimers}

interior are not considered to contribute to $\Sigma$ since they are poorly accessible for DNA molecules. Moreover, screening effects are expected to play an important role and to increasingly dominate when the solution $\mathrm{pH}$ is reduced ${ }^{43}$. The $\mathrm{pH}$ dependence of $\Sigma$ is plotted in Figure 6-18. Changing $\mathrm{pH}$ from high $\mathrm{pH}$ to neutral conditions, $\Sigma$ is monotonically increasing. Independent of dendrimer type or generation, $\Sigma$ reaches its maximum value at $\mathrm{pH}=7$ when all primary amine groups are protonated. Further reducing $\mathrm{pH}$ results in a (slight) decrease in $\Sigma$, since $Q_{\mathrm{I}}$ stays constant and $O$ is increasing due to the successive dendrimer swelling. In the inset of Figure 6-18, maximal surface charge densities $\Sigma$ are contrasted to those known from histone octamers and of fatty acid monolayers, which represent the maximum density that can be achieved by packing of hydrocarbonic chains. ${ }^{192}$ Ranges of $\Sigma$ obtained here for dendrimers clearly comprise the range of surface charge densities known from histone proteins.

\subsection{Conclusions}

Owing to their highly defined architecture, dendrimers are ideal agents for manifold applications. From the results presented here, it can be concluded that for most practical purposes, dendrimers can be viewed as compact, spherical objects with a smeared surface region. Comparing results from PPI and PAMAM dendrimers allows for the conclusion that scaling laws and structural organizations are similar for both types of dendrimers. For the first time, the phenomenon of charge-induced dendrimer swelling has been quantified in detail for dendrimers of a wide range of generations. I expect that the observed, highly predictable response of dendrimers to superimposed electrostatics is a general behavior, which may be found for all types of dendrimers. Possible conformational changes of dendrimers upon charging and the possibility of addressing them externally through varying $\mathrm{pH}$ conditions are most certainly very promising areas of research. The high level of control over the dendrimer size, shape, density, and charge makes these compounds perfectly suited for studying DNA compaction. 


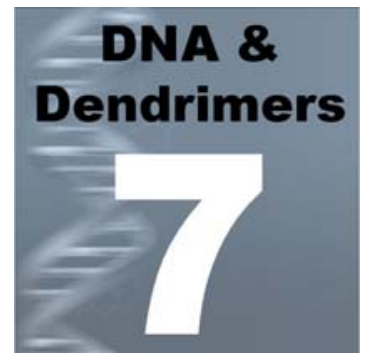

\section{DNA COMPACTION: DENDRIMERS OF INTERMEDIATE SIZE}

In vivo DNA compaction demonstrates nature's sophisticated strategies for packing, protection, and exchange of genetic information. ${ }^{14,15,193}$ In viruses, the DNA compaction usually requires multivalent salts and small organic cations, such as spermidine or spermine, whereas in eukaryotic cells histone proteins play the prominent role. There, the basic unit of organization is the nucleosomal core particle: eight histone molecules form a core particle of $5.5 \mathrm{~nm}$ in diameter and the DNA wraps approximately 1.7 times around that core forming a solenoid of $10 \mathrm{~nm}$ in width. ${ }^{22}$ Although the main driving force for condensation has been identified to be of electrostatic nature, little is known about what determines the stability of the observed, specific structures. ${ }^{10,20}$ Fundamental questions are still unanswered. In how far determine the size and the shape of the histone octamer, how the DNA wraps around it and thus the amount of length compaction? Are specific charge patches on the histone octamer required for a DNA wrapping? Does a uniform spherical charge distribution work as well? In order to answer such questions, it may be useful to consider model systems that are much simpler than the histone/DNA complexes while, at the same time, retaining some of the important characteristics of the histone octamers/DNA fibers.

In this chapter, the dynamic assembly of DNA condensates by cationic dendrimers having an intermediate size and charge that is conveniently between that of small multivalent organic cations and larger histone-like proteins is analyzed. In chapter 6 , dendrimer properties have been determined in great detail and are now used to mimic the influence of electrostatic interactions on DNA compaction in vivo. 
Performing measurements under laminar flow conditions in a hydrodynamic focusing device, the microfluidic setup ensures a non-equilibrium ascent of reactant concentrations and allows for probing the real-time dynamic evolution of supramolecular interactions between DNA and dendrimers. The resulting progression of DNA compaction is detected online using microfocused small-angle X-ray diffraction and confocal Raman microscopy. The combination of both, the structural information resulting from small angle X-ray scattering measurements and the chemical sensitivity of Raman imaging, allows for a detailed insight into the characteristics and the dynamics of self-assembled dendrimer/DNA condensates.

\subsection{Confocal Raman imaging of PPI 4 induced DNA compaction}

\subsubsection{Raman spectrum of PPI 4/DNA complexes}

Raman spectroscopy is used for sensing molecular interactions of PPI dendrimers generation 4 and DNA. The Raman spectrum of PPI 4/DNA complexes shown in Figure 7-1 is an accumulated average of 50 exposures of $150 \mathrm{~s}$ each. The spectral resolution is $6 \mathrm{~cm}^{-1}$. According to the discussion of the Raman spectrum of DNA in chapter 5.2.2, Figure 7-1 shows a wave number region of $600-1700 \mathrm{~cm}^{-1}$.

The Raman spectrum of dendrimer/DNA condensates is largely consistent with the sum of intensities of corresponding Raman bands in the individual dendrimer and DNA spectra as can be readily seen in the inset picture showing the difference spectrum. However, a distinct reduction in intensity of the most prominent phosphate group bands at 782 and $1087 \mathrm{~cm}^{-1}$ is revealed indicating that these negatively charged groups along the DNA backbone are the main targets of dendrimer amine groups. This reflects the fact that an electrostatic attraction between both molecules is the driving force of DNA compaction. Consistently, all dendrimers are expected to have similar impact on the Raman spectra of corresponding dendrimer/DNA condensates. Although the Raman band at $782 \mathrm{~cm}^{-1}$ shows a more prominent change in intensity during compaction, we want to focus on the Raman band at $1087 \mathrm{~cm}^{-1}$, which arises from a symmetric stretching vibration of $\mathrm{PO}_{2}^{-}$moieties and is easily distinguishable from other Raman bands of B-form DNA in its vicinity (see chapter 5.2.2). 


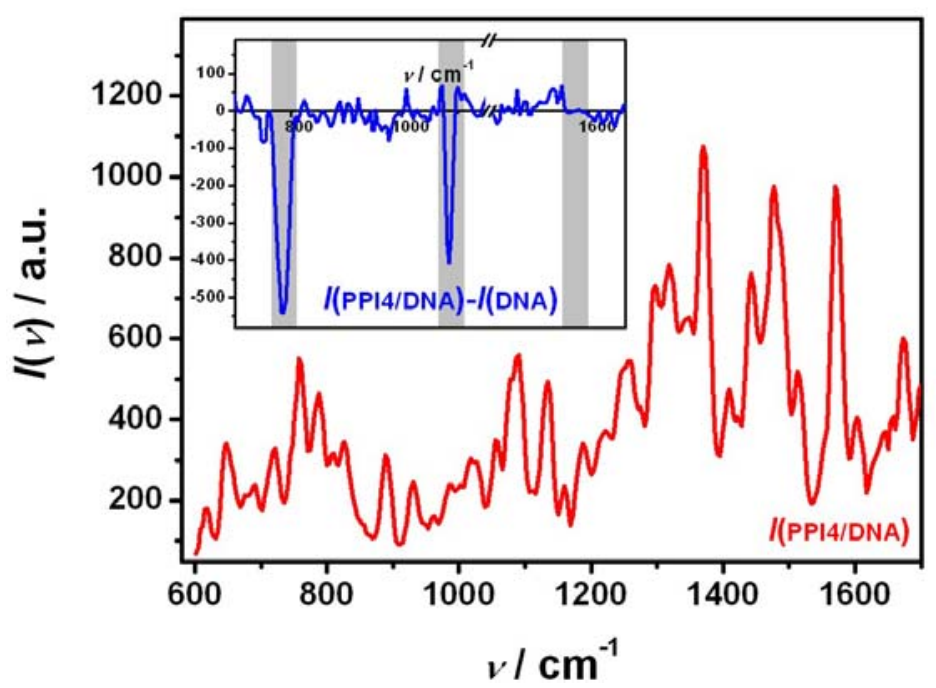

Figure 7-1: Raman spectrum of PPI4/DNA condensates. The inset shows the difference spectrum between PPI 4/DNA and DNA. Wavenumber positions of 782,1087 , and $1576 \mathrm{~cm}^{-1}$ are annotated.

It is known that the complexation of DNA by polyamines preserves and stabilizes the B-form duplex structure of DNA without significantly perturbing base pairing or base stacking interactions of DNA. ${ }^{146}$ Thus, Raman bands belonging to base vibrations are almost unaffected by the complexation. Accordingly, the intensity of the prominent Raman band at $1576 \mathrm{~cm}^{-1}$, which arises from vibrations of the bases guanine and adenine, can be chosen as reference. Therefore, the reduction in intensity of the Raman band at $1087 \mathrm{~cm}^{-1}$ relative to the reference intensity at $1576 \mathrm{~cm}^{-1}$ can be used as a benchmark for DNA compaction reactions. Comparing intensities of the Raman band at $1087 \mathrm{~cm}^{-1}$ in DNA and PPI 4/DNA spectra exhibits a reduction of $30 \%$.

\subsubsection{Real-time monitoring of DNA compaction in microflow}

The knowledge of the Raman spectrum of dendrimer/DNA aggregates is applied for a systematic Raman monitoring of DNA compaction and for investigating its nonequilibrium structure formation dynamics under hydrodynamic focusing conditions. The hydrodynamic focusing device used here consists of two perpendicularly crossed microchannels having a depth of $300 \mu \mathrm{m}$ and a width of $150 \mu \mathrm{m}$. A semi-diluted aqueous DNA solution with a concentration of $c_{\mathrm{DNA}}=2.5 \mathrm{mg} \mathrm{mL}^{-1}$ is injected in the main channel with a mean flow velocity of $u_{\mathrm{DNA}}=200 \mu \mathrm{m} \cdot \mathrm{s}^{-1}$. Hydrodynamic focusing of the DNA solution is initially performed by injecting pure water from the side channels with a velocity of $u_{\text {side }}=4 u_{\text {DNA }}$. After establishing a stationary flow, PPI 4 
dendrimers are continuously added to both side streams. Dendrimer concentrations in the side channels are determined by the co-flow of aqueous dendrimer solution and water into upstream T-valves, which allow for an online control of the dendrimer concentration (see Figure 4-8). The dendrimer concentration can be varied from 0 (only water) to $10 \mathrm{mg} \mathrm{mL}^{-1}$ (only aqueous PPI 4 solution). Hydrodynamic focusing experiments are performed at $\mathrm{pH}=8.5$. Important dendrimer properties are listed in chapter 6.1.

Subsequently to the intersection, the interaction of dendrimers with DNA can be observed along the outlet channel. The flow in microchannels is laminar (Reynolds number $\operatorname{Re}<1$ ), meaning that mixing of components is diffusion controlled. ${ }^{56}$ Positively charged dendrimers diffuse into the DNA stream creating a well defined gradient of chemical reactants for the negatively charged, pre-elongated DNA molecules. Flow velocities are chosen such that a concentration gradient of the reactants extends along the measurable length of the device. It follows that the complex composition varies at every accessible point along the reaction channel reflecting a different extent of DNA compaction. In addition, mixing of components in this well defined manner reduces the likelihood of the creation of kinetically trapped phases.

High resolution 2D confocal Raman images of the hydrodynamic focused DNA stream are recorded at fixed wave numbers of $1087 \mathrm{~cm}^{-1}$ and $1576 \mathrm{~cm}^{-1}$, respectively, by laterally $(x-y)$ rastering the sample through the excitation spot and detecting the Raman signal with the avalanche photodiode detector (APD) (chapter 2.1). Raman intensities $I_{1087}(x, y)$ recorded at the wavenumber $v=1087 \mathrm{~cm}^{-1}$ are divided by corresponding values $I_{1576}(x, y)$ obtained at $v=1576 \mathrm{~cm}^{-1}$ for every $(x, y)$-position. The resulting image is displayed in Figure 7-2 (bottom) showing already reacted areas. Only half of the device is given since the pattern is symmetric.

Combining microfluidics with confocal Raman microscopy allows for direct visualization of mutual effects of flow conditions and biomaterial properties. Finite element simulations of physical conditions inside microchannels are performed in order to model the experimental situation (chapter 2.3). A detailed comparison of simulation and experiment allows for improved understanding. The diffusion constant $D_{\text {PPI4 }}$ of PPI 4 dendrimers and the viscosities of the DNA solution, $\eta_{\mathrm{DNA}}$, and of the formed PPI 4/DNA assemblies, $\eta_{\text {complex }}$, are used as fit parameters in the simulations to match the experimental results, i.e. the shapes of the hydrodynamically focused center stream and of formed dendrimer/DNA assemblies. All other parameters such as the channel geometry and flow rates are known. The diffusion of long chain DNA molecules, which are preferentially oriented perpendicular to the diffusion direction, into the side 


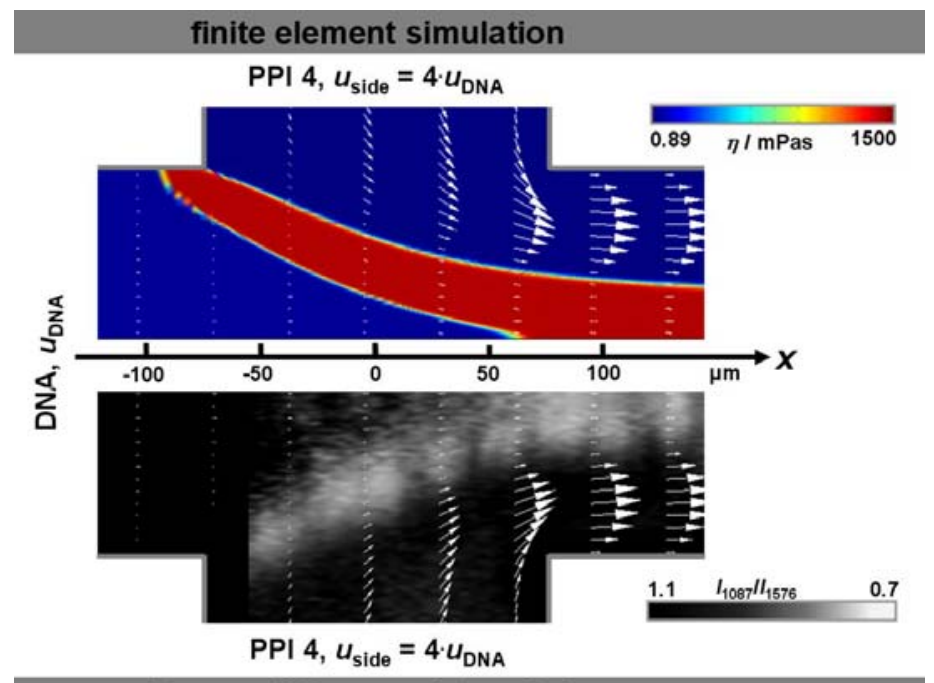

Raman-difference intensity image

Figure 7-2: Real-time monitoring of dendrimer induced DNA compaction in a hydrodynamic focusing device. The Raman-difference intensity indicates the compaction yield (bottom). In the simulation (top), the product of the complexation reaction appears in the diffusion cone of side and main stream components due to its highly increased viscosity.

streams can be neglected since the mobility of DNA macromolecules $\left(D_{\mathrm{DNA}} \approx 2 \cdot 10^{-12} \mathrm{~m}^{2} \cdot \mathrm{s}^{-1}\right)^{194}$ is orders of magnitude lower than that of PPI 4 dendrimers.

The simulated flow field is given in Figure 7-2 (top). For a direct comparison with experimental results, the modeled velocity profile (arrow plot) in the hydrodynamic focusing device is overlaid to the Raman image (bottom). The product of the complexation reaction appears in the diffusion cone of side and main stream components. In simulations, dendrimer/DNA complexes can be best visualized by utilizing the fact that a strong increase in local viscosity is connected to the complexation reaction (Figure 7-2 top). Very small deviations of simulation results from the experimentally recorded shape are observed in the first third of the crossing area where the center stream is slightly bulged into the side channels. These deviations are ascribed to effects of the walls at the bottom and the top of the microchannel that are not considered in the 2D simulations. ${ }^{195}$ Apart from this detail, the experiment and the simulation show excellent agreement.

Analyzing complex materials in hydrodynamic focusing devices provides a method to evaluate the viscosity of minute quantities of very viscous fluids (chapter 5.3.1). PPI 4 dendrimers diffusing into the DNA stream induce a complex formation, which leads to an increase of the local viscosity. To characterize the complex formation, the viscosity has to be coupled to local concentrations of PPI 4 and DNA and its functional dependence has to be described by a single function. As has been demonstrated for the 
self-assembly of collagen, the situation can be well approximated by $\eta=\eta_{\text {water }}+\eta_{\text {DNA }}+\eta_{\text {complex }}{ }^{195}$ Finite element simulations include the solution of a highly complex system of coupled differential equations. To account for a good numerical handling of this system, it is necessary to avoid discontinuities in $\eta$ and its derivatives, in particular at the transition from the uncomplexed materials of low viscosity to the highly viscous dendrimer/DNA complexes. In the simulations, this is achieved by utilizing the sharp but continuous concentration profile of DNA: starting at a threshold value of $N / P=0.05, \eta_{\text {complex }}$ is increasing proportional to the cube of the normalized DNA concentration $c_{\mathrm{DNA}} / 2.5 \mathrm{mg}^{-1} \mathrm{~mL}^{-1}$. Although this power-law dependence is only fulfilling a mathematical requirement and has no strict physical meaning, simulations with varying starting conditions have shown that the DNA concentration profile is dominating over the power-law, whose exact magnitude has almost no effect on the system.

The viscosity of the DNA solution and of dendrimer/DNA condensates are fitted to $\eta_{\text {complex }} \approx 1500 \eta_{\text {water }}=1.33 \mathrm{~Pa} \cdot \quad$ and $\quad \eta_{\text {DNA }}\left(2.5 \mathrm{mg}^{-1}\right) \approx 35{ }^{-1} \eta_{\text {water }}=31.2 \mathrm{mPa} \cdot \mathrm{s}$, respectively. The viscosity of formed biocomplexes is more than 1500 times higher than $\eta_{\text {water. }}$ These results are on the same order of magnitude as results known from other polymer hydrogels. ${ }^{195-197}$ However, to directly verify the consistency of simulation results and the experimental situation, the viscosity of $2.5 \mathrm{mgmL}^{-1} \mathrm{DNA}$ solution has been additionally determined by conducting bulk measurements using an Ubbelohde-viscometer. The obtained result of $\eta_{\mathrm{DNA}}\left(2.5 \mathrm{mg}^{-1} \mathrm{~mL}^{-1}\right)=(30.8 \pm 1.5) \mathrm{mPa} \mathrm{s}$ agrees remarkably well with simulation results. Hence, microfluidic experiments in combination with finite element simulations represent a powerful method to analyze the complete process of DNA complex assembly in terms of the solution viscosity. In particular, this is noteworthy because involved solution viscosities range over five orders of magnitude and are coupled via complexation reactions. The influence of the viscosity on the flow fields in microchannels have been discussed in detail in chapter 5.3.1.

The dendrimer induced DNA complexation results in the formation of highly viscous PPI 4/DNA complexes, through which dendrimers have to successively diffuse. Adjusting simulations to match experimental results yields information related to the diffusion process. According to the Stokes-Einstein equation, the viscosity and the diffusion coefficient are reciprocal:

$$
D=\frac{k T}{6 \pi \eta R} .
$$


Taking into account the high viscosity of dendrimer/DNA complexes, this would imply that the dendrimer diffusion into the DNA solution should be about four orders of magnitude slower than in a corresponding water-water system without viscosity raising DNA. However, large positive deviations from the ideal Stokes-Einstein behavior are known for diffusion in crowded and strongly interacting macromolecular systems, which are still a huge challenge to our understanding. Despite the importance of microrheological properties in biology and in industrial applications, there is no single theory available explaining diffusion phenomena in complex, structured fluids. ${ }^{198}$ Large polymeric molecules strongly increase the solution viscosity even at relatively low volume fractions. Contrary to the movement of large objects however, the viscosity that determines the movement of very small objects in such complex fluids is often close to that of pure solvent. ${ }^{198}$ This is due to the fact that the rather uneven space occupancy creates fluctuating, solvent-filled regions in the polymeric network, through which probe molecules can diffuse considerably faster than it would be predicted from bulk viscosity. $^{199-205}$ Besides anisotropy effects, electrostatic ${ }^{199,206}$ and elastic ${ }^{207}$ contributions are significantly affecting diffusivity of probe molecules in hydrogels yielding both reduced diffusion coefficients and diffusion coefficients even larger than observed in the pure solvent. ${ }^{208}$

The complex interplay of these effects is accounting for the observed value of $D_{\text {PPI } 4} \approx 1.6 \cdot 10^{-10} \mathrm{~m}^{2} \mathrm{~s}^{-1}$. This rather corresponds to the result one would obtain from the Stokes-Einstein relation $\left(r_{\mathrm{PPI} 4}=1.4 \mathrm{~nm}\right.$ at $\left.\mathrm{pH}=8.5\right)$ under purely aqueous conditions. Strikingly, using hydrodynamic focusing, dynamical parameters of chemical reactions in such complex, interacting systems can be quantified, which is essential in chemical engineering, chemistry, and biology.

Since the whole system is dominated by laminar flow, each position along the outlet channel represents a steady state in the reaction evolution. Owing to the diffusive mixing behavior, different local concentrations exist along and perpendicular to the hydrodynamically focused DNA stream. Changes in the relative charge ratio $N / P$, which is known to influence the structure of formed aggregates, ${ }^{53}$ are correlated with these concentration variations. Here, $N$ denominates the number of positive amine charges of dendrimers, whereas $P$ is the number of negative phosphate charges of the DNA backbone. Owing to the laminar flow and the diffusive mixing, different $N / P$ ratios and therefore different states of aggregation are observable along the outlet channel. Accordingly, different time frames of the compaction reaction are accessible by varying flow velocities and/or the observation position along the main channel. From simulation data, local experimental conditions such as the velocity and the concentration can be obtained at each position. 
(a)

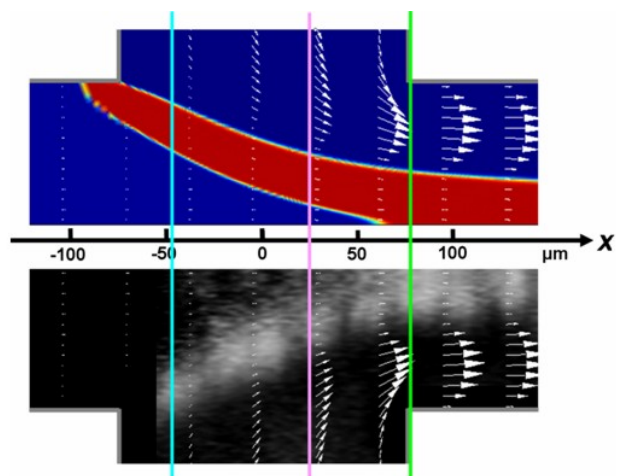

(c)

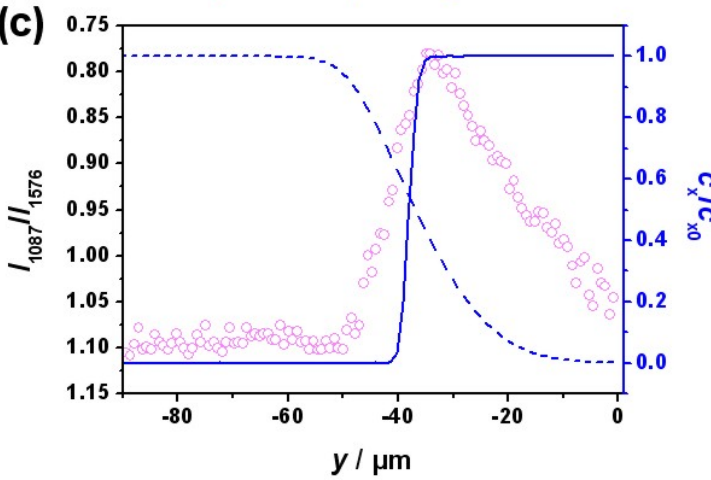

○ $I_{1087} I_{1576}$

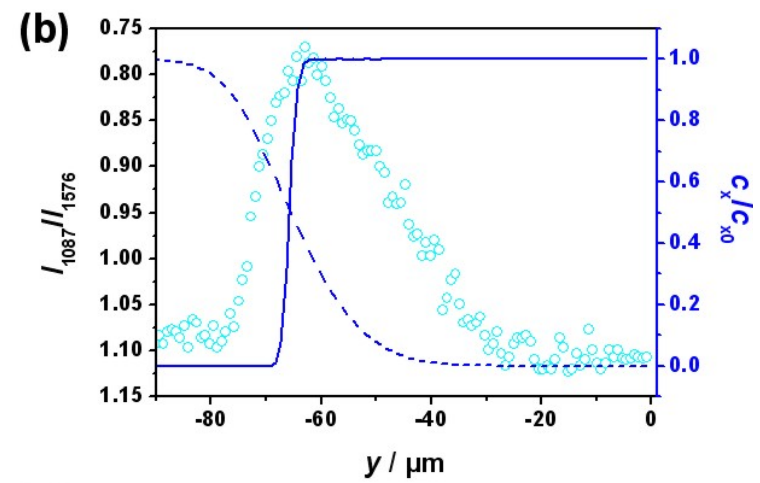

(d)

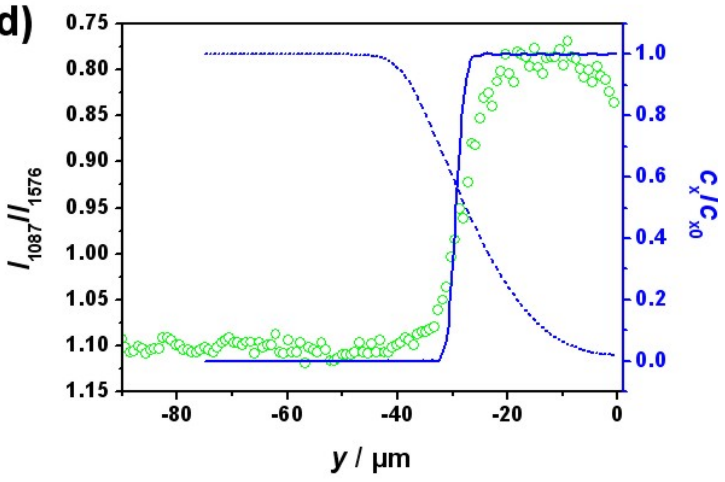

DNA - - PPI 4

Figure 7-3: Line scans perpendicular to the reaction channel at three positions $x=-50,25$ and $75 \mu \mathrm{m}$. Corresponding data are shown in (b)-(d). Dashed and solid lines give the normalized concentration profiles $c_{\mathrm{PPI} 4} / c_{\mathrm{PPI}, 0}$ and $c_{\mathrm{DNA}} / c_{\mathrm{DNA}, 0}$, respectively.
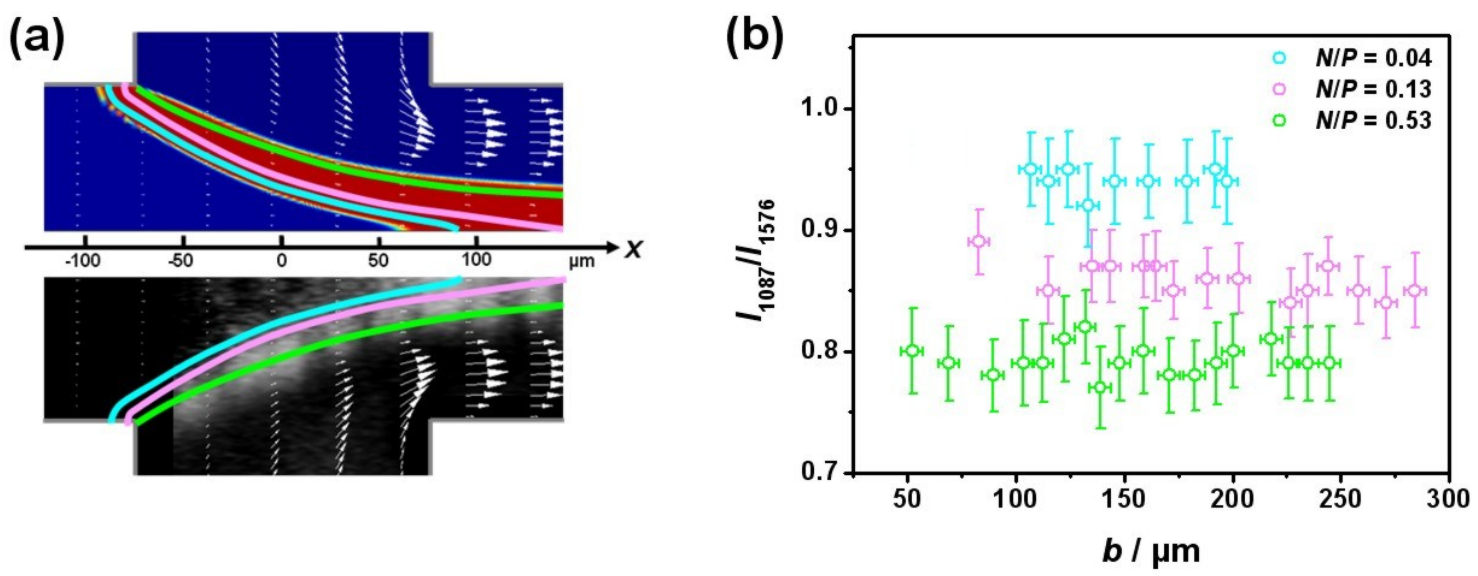

Figure 7-4: (a) Lines with constant $N / P$ ratio at each position. (b) Courses of the Raman intensity ratio $I_{1087} / I_{1576}$ along the three equi-concentration lines depicted in (a). 


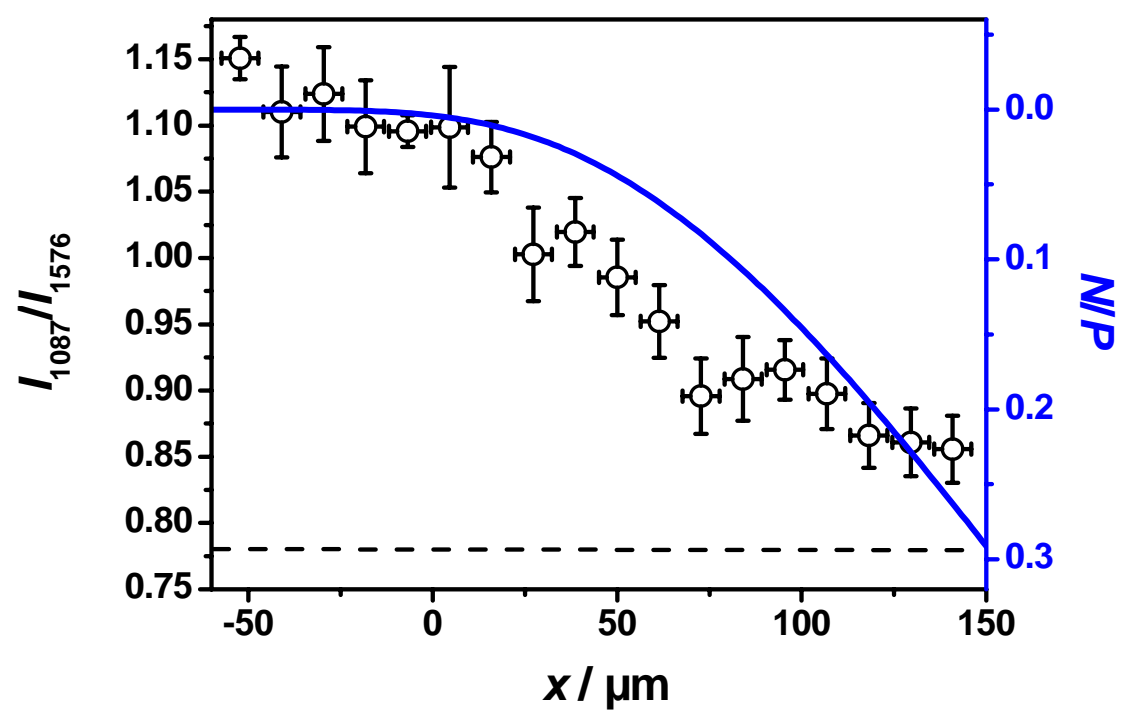

Figure 7-5: Monitoring the DNA complexation along the streamline in the center of the reaction channel $(y=0)$. The $N / P$ axes has been inverted to allow for improved comparability.

Experimental and simulation data are compared by analyzing line scans at specific positions in the channel. For each line scan, the Raman signal is averaged over seven lines. From Raman spectra the ratio of intensity of the two Raman bands at $1087 \mathrm{~cm}^{-1}$ and $1576 \mathrm{~cm}^{-1}$ for pure (non-compacted) DNA is known to be $I_{1087} / I_{1576} \approx 1$.1. Adding PPI 4 molecules, the negatively charged phosphate groups are complexed by positively charged dendrimer sites and the ratio of intensity of the two Raman bands decreases to a final value of $I_{1087} / I_{1576} \approx 0.78$. In Figure 7-3a, three positions $x=-50,25$ and $75 \mu \mathrm{m}$ are marked by colored lines. Corresponding line scans are shown in Figure 7-3b-d. $I_{1087} / I_{1576}$ is given by open circles, solid lines show the normalized DNA concentration $c_{\mathrm{DNA}} / 2.5 \mathrm{mg}^{-1}$, and dashed lines represent the corresponding course of the normalized dendrimer concentration $c_{\mathrm{PPI}} / 10 \mathrm{mg} \mathrm{mL}^{-1}$.

Dendrimers are successively diffusing into the DNA stream and condensing to the negatively charged phosphate groups of the DNA backbone. $y$-regions with a reduced intensity ratio $I_{1087} / I_{1576}$ correspond to already complexed regions of the DNA stream. Figure 7-3 shows that the decrease in intensity is directly correlated with changes in concentrations owing to diffusion. The asymmetry in the reaction zone is due to different diffusion rates of the two reagents. This is also reflected in the different courses of normalized concentrations of DNA and PPI 4. Whereas Figure 7-3d $(x=75 \mu \mathrm{m})$ shows a perfect match of concentration courses and Raman intensity ratio, at $x=25 \mu \mathrm{m}$ (Figure 7-3c) slight deviations between experimental data and simulation 

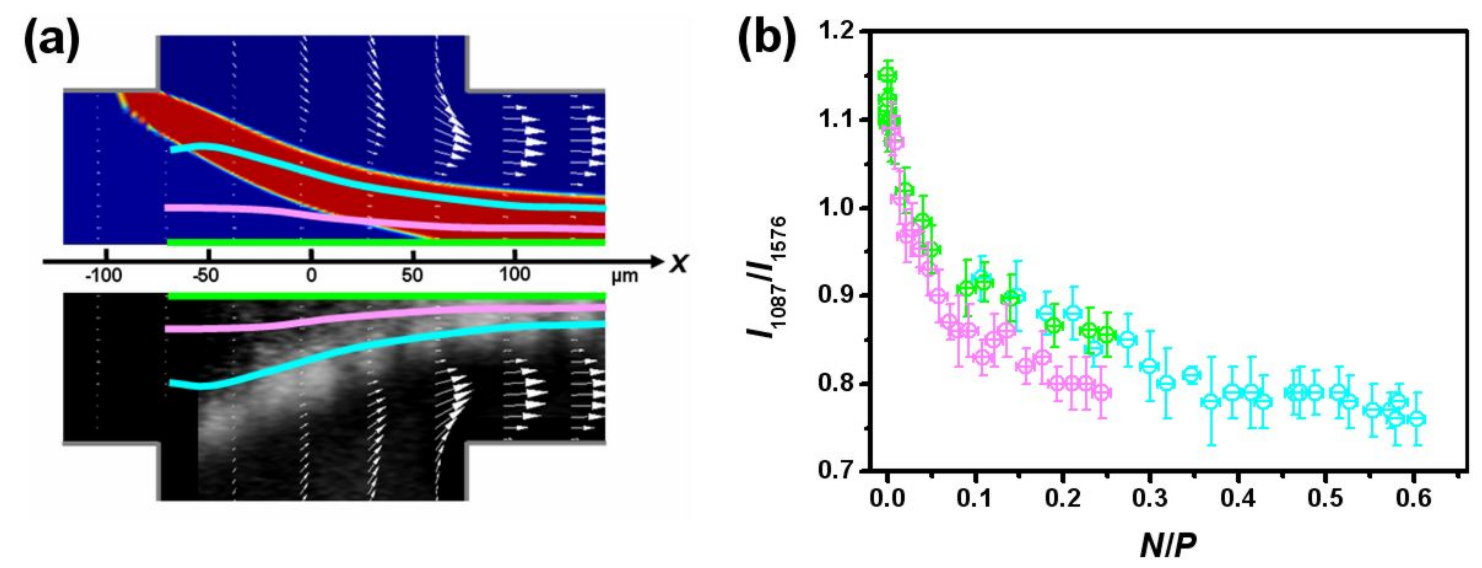

Figure 7-6: $\quad N / P$ dependence of the Raman intensity ratio $I_{1087} / I_{1576}$ (b). Data sets are obtained along the three streamlines depicted in (a).

are observable, which become more pronounce at $x=-50 \mu \mathrm{m}$ (Figure 7-3b). These deviations are a result of the slight bulging of the more viscous DNA center stream into the side channels as has been discussed above. For each of the three $x$-positions, the final ratio of intensity $I_{1087} / I_{1576} \approx 0.78$ is reached at $N / P=(0.55 \pm 0.05)$. This finding shows that experimental results as well as the numerical description are consistent.

Experimental and simulation data are further compared by analyzing physical properties along different lines exhibiting identical $N / P$ ratios at all positions. Three such lines are shown in the inset picture in Figure 7-4 corresponding to $N / P=0.04$, 0.13 and 0.53 . Since $c_{\mathrm{DNA}}$ exhibits only slight deviations from $2.5 \mathrm{mg} \mathrm{mL}^{-1}$ at positions analyzed, the lines are equi-concentration lines corresponding to $c_{\mathrm{PPI}}=0.3,1$ and $4 \mathrm{mg} \mathrm{mL}^{-1}$, respectively. From the Raman image, the intensity ratio $I_{1087} / I_{1576}$ at positions along each line are extracted. Figure $7-4 \mathrm{~b}$ shows the dependence of $I_{1087} / I_{1576}$ on the position $b$ along each line given in Figure $7-4 a$. For each $N / P$ ratio, $I_{1087} / I_{1576}$ is almost constant showing no systematic or significant changes. This finding indicates that the experimental parameters employed here are such that the reaction rate of PPI 4/DNA complexation is much faster than the observations and that the diffusion time scale is much larger than the reaction time scale.

The evolution of DNA compaction mediated by PPI 4 dendrimers can also be monitored along different stream lines. In Figure 7-5, this is exemplarily shown along the streamline in the center of the reaction channel. The Raman signal is averaged over seven lines and plotted in dependence of the position along the streamline, i.e. the $x$ coordinate. Raman data are contrasted to the $N / P$ ratio calculated at each position from local DNA and PPI 4 concentrations. Figure 7-5 shows that the decrease in intensity is directly correlated with changes in $N / P$ and therefore with changes in concentrations. 
Following the center streamline, the final ratio of intensity $I_{1087} / I_{1576} \approx 0.78$ is not reached in the observed reaction area.

To analyze the evolution of DNA complexation in more detail, in Figure 7-6b further data obtained from analyzing DNA complexation along two additional streamlines (depicted in Figure 7-6a) are presented. Data obtained along all three streamlines cover the whole range of $I_{1087} / I_{1576} \approx 1.1 \ldots 0.78$ and are consistent to each other collapsing on a single curve. According to results from line scans perpendicular to the reaction channel, $I_{1087} / I_{1576} \approx 0.78$ is again found to correspond to $N / P \approx 0.55$. Although PPI 4 dendrimers are continuing to diffuse into the DNA stream, the intensity ratio $I_{1087} / I_{1576}$ remains constant indicating that the maximum amount of accessible phosphate groups is complexed by dendrimer amine groups. The fact that an excellent agreement for all analyzed positions is found that emphasizes the validity of the assumptions made for the simulations.

Altogether, the experimental system can be well described by finite element simulations. The diffusive mixing leads to a stable reaction gradient, which induces the complexation. The complexation can be directly visualized by a decrease in Raman intensity ratio coinciding with a dramatic increase in the solution viscosity. To obtain structural information of formed PPI 4/DNA aggregates, small angle X-ray microdiffraction is used.

\subsection{X-ray microdiffraction measurements of PPI 4 induced DNA compaction}

Small angle X-ray scattering plays a prominent role as a characterization technique for biomaterials and biological objects in microfluidic systems ${ }^{105}, 107$ since it probes relevant length scales (on the order of $\mathrm{nm}$ ). In particular, spatially resolved microfocused X-ray scattering ${ }^{60}$ in hydrodynamic focusing microdevices provides new perspectives in studying interaction dynamics of DNA under tunable conditions. Previously, rich phase diagrams of stable PPI 4/DNA ${ }^{53}$ and PAMAM 4/DNA complexes $^{54}$ have been reported, identified using X-ray diffraction. However, the use of synchrotron radiation can damage the sample and, typically, bulk samples are prepared in advance and require large amounts of expensive biomaterials. Perhaps most importantly, bulk experiments are not amenable to online variation of DNA condensation conditions as are those reported here. Using hydrodynamic focusing microdevices, the width of the focused DNA stream can be adjusted by the ratio of 


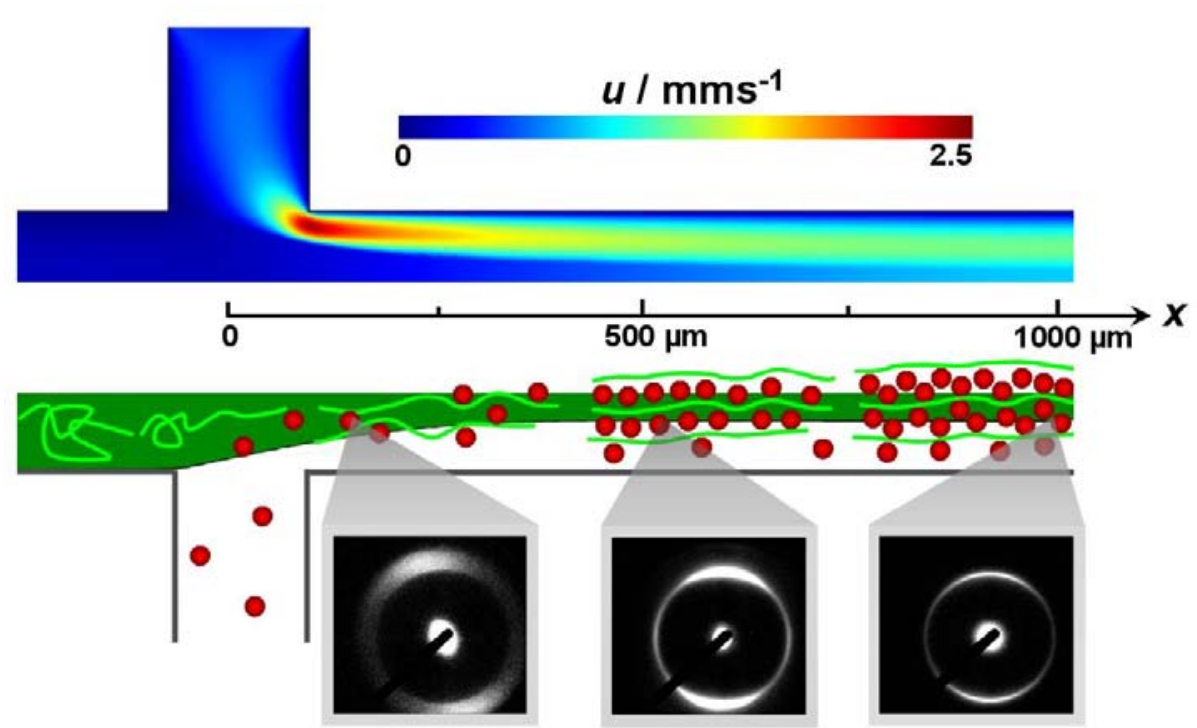

Figure 7-7: Close-up schematics of the evolution of dendrimer induced DNA compaction in a hydrodynamic focusing device (bottom). Representative 2D X-ray images recorded along the reaction channel are shown. The simulated velocity field (top) is shown for comparison. Relevant length scales are annotated.

flow rates of the main channel and the side channels. Therefore, mixing and concentration distributions in the reaction channel can be adjusted by changing the width of the hydrodynamically focused DNA stream as well as flow velocities in all channels. Consequently, interaction dynamics depending on the concentration distribution can be spatially separated in steady state flow.

Structural information can be obtained from the effective structure factor $S$, which is obtained by revising the scattering intensity $I(q)$ by the normalized intensity of a dilute sample. Qualitatively, peaks of the structure factor at distinct $q$ positions can be interpreted as "Bragg reflections" from planes of particles separated by a certain mean nearest-neighbor distance $d \sim q^{-1}$.

\subsubsection{PPI 4/DNA mesophase formation}

In a first set of SAXS experiments, ${ }^{209}$ a $10 \mathrm{mg}^{-1} \mathrm{~mL}^{-1}$ DNA solution is injected in the main channel with a mean velocity of $u_{\mathrm{DNA}}=100 \mu \mathrm{m}^{-1}$ and $20 \mathrm{mg}^{-1} \mathrm{~mL}^{-1}$ PPI 4 dendrimer solutions are added to the side channel with a mean velocity of $u_{\text {PPI }}=4 u_{\text {DNA }}$. The well defined, purely diffusive mixing of components reduces the likelihood of the creation of kinetically trapped phases. Experimental conditions are 

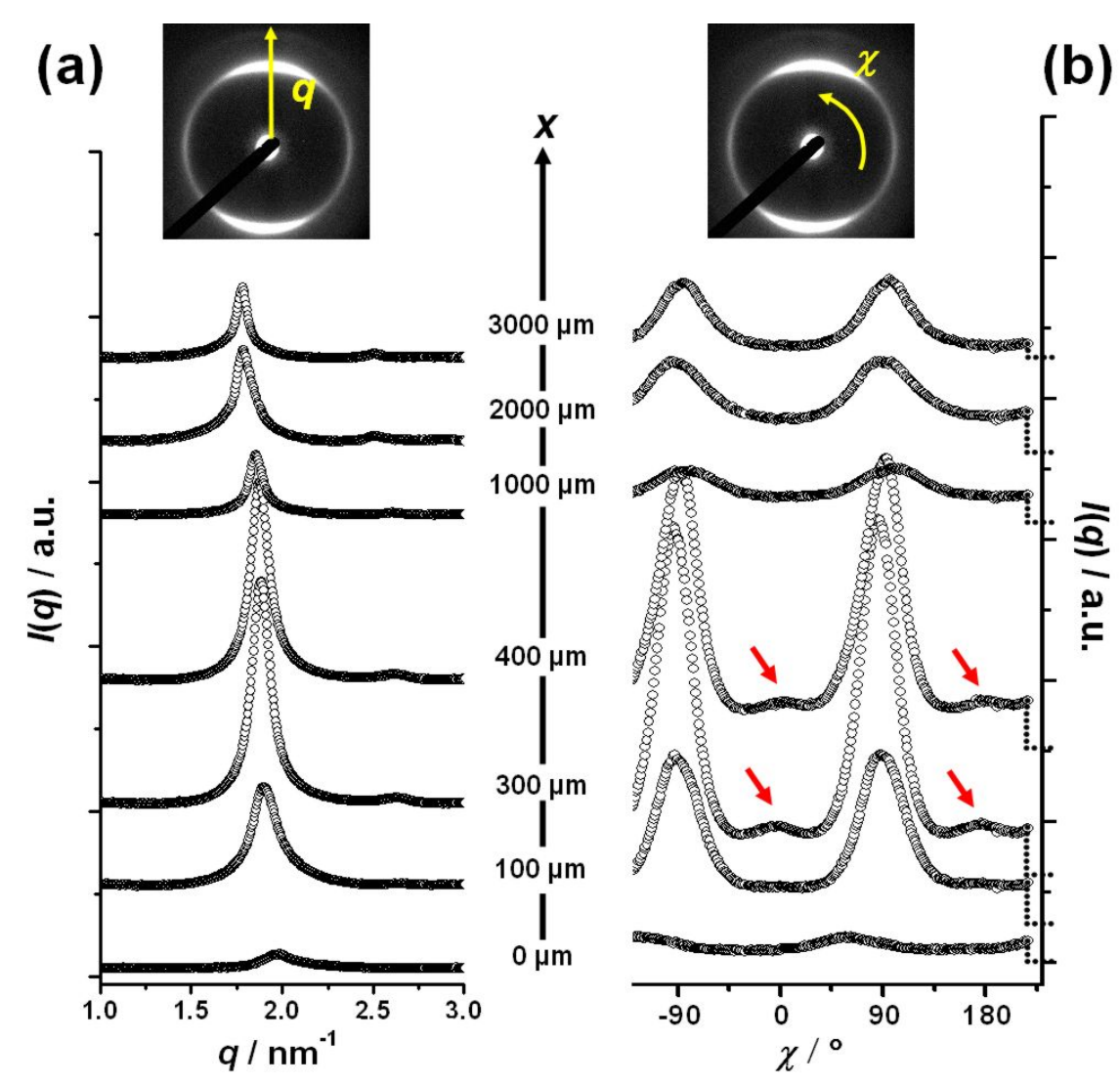

Figure 7-8: Radial averaging (a) and azimuthal integration along the peak $q_{0}$ (b) for the same positions $x$ along the microchannel revealing preferential orientation at $\chi= \pm 90^{\circ}$. Baseline intensities are annotated on the righthand side (dotted lines). ${ }^{209}$

chosen to result in a final charge ratio $N / P<6$ at the furthest measurable point of the device $(x \approx 3 \mathrm{~mm})$. A lateral scanning of the sample allows for spatially resolved small angle X-ray diffraction experiments along the microfluidic channels. The positional accuracy of absolute $x$ and $y$ coordinates in the microdevice is on the order of the beam size $(20 \mu \mathrm{m})$. CCD images are collected with exposure times of 30-120s per position.

Representative X-ray diffraction patterns of PPI 4 induced DNA condensation measured at different distances $x$ from the confluence center are shown in Figure 7-7 (bottom). It is important to note that all images reveal oriented diffraction rings. Owing to a concurrent orientation during the assembly process in microflow, the characterization of biomolecular materials, which are difficult to crystallize and normally form liquid-crystalline structures, is significantly improved. ${ }^{57,63,64}$ This is a clear advantage of using flow to assemble dendrimer/DNA complexes. Qualitative differences (i.e. in the peak width and in the azimuthal orientation) between these images are readily seen. 

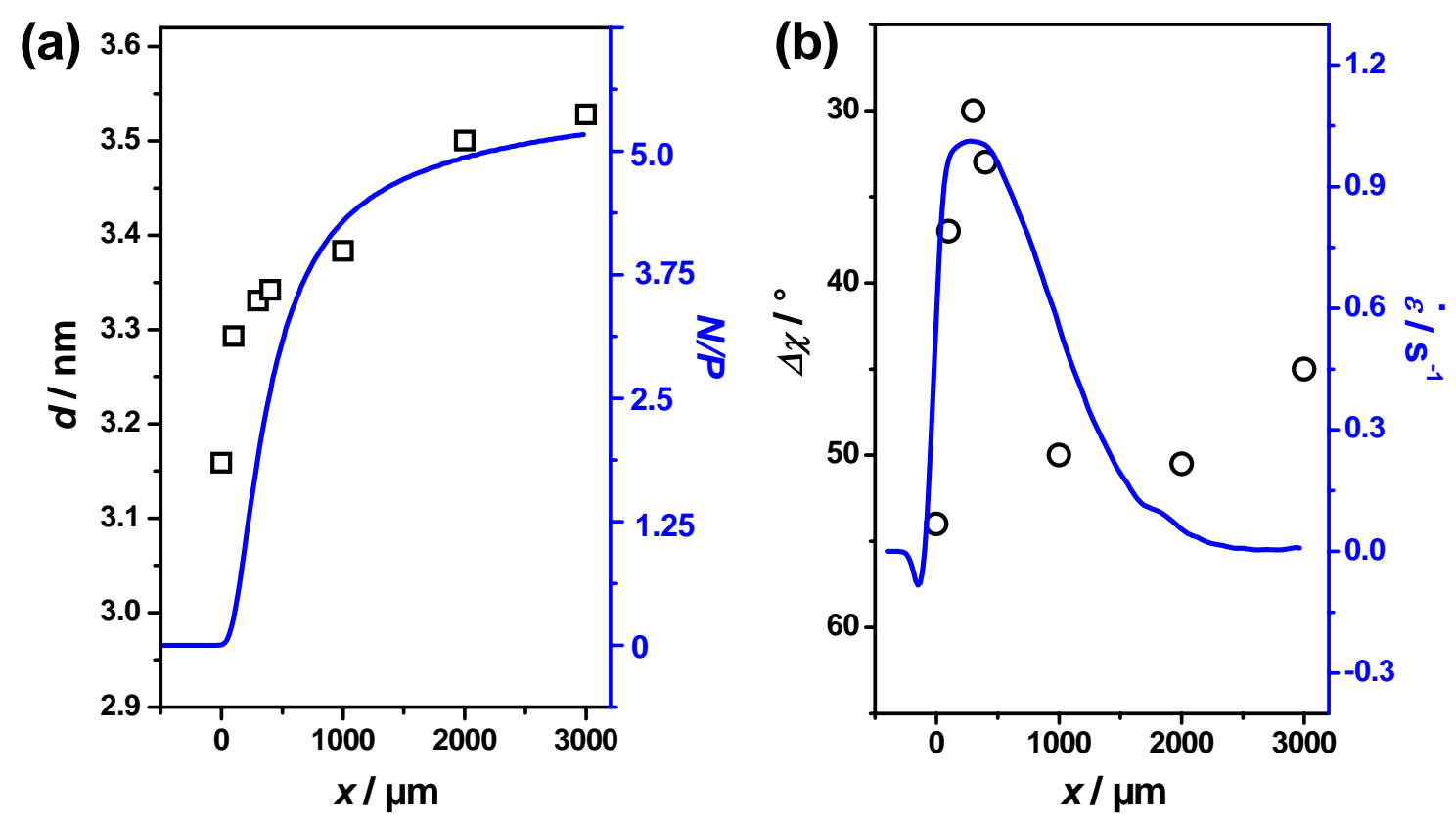

Figure 7-9: The real space distance $d$ of the 2D square lattice (a) and the degree of preference $\Delta \chi$ showing good agreement with the calculated strain rate $\dot{\varepsilon}$ (solid line) in dependence of the $x$ position along the microchannel.

A quantitative evaluation of Raman data obtained from measuring the non-equilibrium DNA compaction under hydrodynamic focusing conditions shows the enormous benefit to our understanding of comparing experimental results to simulation data. Accordingly, flow velocity and concentration profiles in the hydrodynamic focusing device are again modeled using parameters consistent to results presented in chapter 5.3 and 7.1.2. ${ }^{210}$ The simulated velocity field is exemplarily shown in Figure 7-7 (top).

Small angle X-ray diffraction data plotted in terms of the reciprocal vector $q$ and obtained via a radial integration of the raw image data are given in Figure 7-8a. ${ }^{61}$ Plots of X-ray data in the figures are offset for clarity. Baseline values of intensity for azimuthal integrations are included in the data plots in Figure 7-8b.

Starting at the confluence center of the microchannels $(x=y=0)$, a single peak at $q_{0}=2.0 \mathrm{~nm}^{-1}$ is identified (lowest curve in Figure 7-8a). Moving the observation position towards larger $x$, which corresponds to larger $N / P$ ratios, the $q_{0}$-peak is shifted towards smaller $q$ values and a second peak $q_{1}$ is concurrently acquired. The ratio $q_{1}=\sqrt{2} q_{0}$ of the two peak positions is consistent with a columnar mesophase with inplane square symmetry.

The lattice constant $d$ of such a unit cell is related to the peak position $q_{0}$ using the relation $d=2 \pi / q_{0}$. An increase in the lattice spacing $d$ is obtained at observation points 


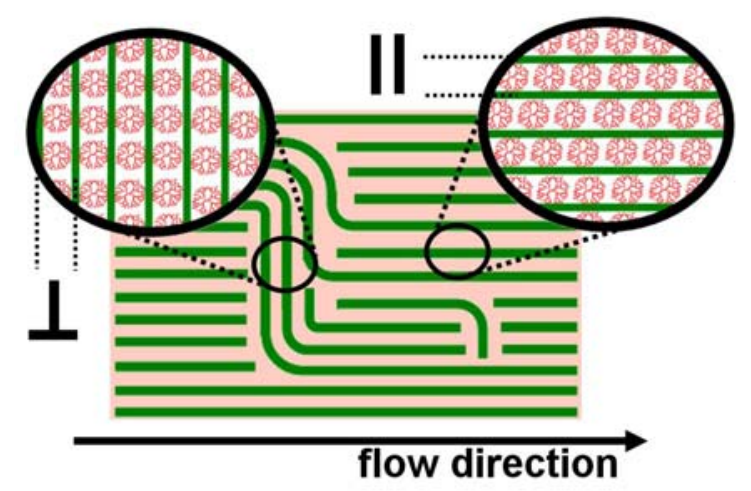

Figure 7-10: Schematic representation of the microdomain orientation in the region of maximum strain.

further downstream. This can be understood by the incorporation of additional dendrimers within the PPI 4/DNA condensate. The increase in $d$ (obtained experimentally) is shown in Figure 7-9a to overlay remarkably well with $N / P$ values provided by finite element simulations (solid line in Figure 7-9a) as a function of the measurement position $x$ in the device. These data are consistent with previously reported bulk X-ray diffraction measurements, ${ }^{53}$ citing a $2 \mathrm{D}$ columnar lattice of PPI 4/DNA condensates for $N / P$ values $2 \leq N / P<6$. The precise diffusive mixing of components in flow uniquely allows visualizing an increase in $d$ over the entire range of columnar mesophases explored here.

The mesoscale alignment of macromolecular assemblies is not easily achieved using standard sample preparation methods. However, an alignment in flow is comparatively straightforward using the setup reported here. At the confluence of the microchannels, the main stream is focused and fluid elements are accelerated. Owing to this extensional flow, DNA molecules as well as dendrimer/DNA assemblies experience an additional hydrodynamic stress, which leads to an orientation along the flow direction. Scans of the intensity along the azimuthal angle $\chi$ on the ring of the $q_{0}$-peak at different channel positions $x$ are shown in Figure 7-8b. Moving from $x=0$ along the reaction channel, all X-ray scans of dendrimer/DNA assemblies show a strong preferential orientation along the flow direction $\left(\chi \approx \pm 90^{\circ}\right)$ down to a position $x=3000 \mu \mathrm{m}$. According to the discussion in chapter 5.3.1, such a superimposed hydrodynamic stress, which can be experienced up to several $1000 \mu \mathrm{m}$ downwards the reaction channel, is expected for experimental situations characterized by a significantly higher viscosity of the main channel solution compared to side channel streams.

The extensional flow leads to a favored orientation of DNA macromolecules parallel to the applied stress (Figure 7-9b). The degree of orientation can be quantified as the full width at half maximum of the azimuthal peaks $\Delta \chi$. Figure $7-9 \mathrm{~b}$ clearly shows a 
minimum value of $\Delta \chi$, corresponding to the highest extent of material orientation, at a position $x=300 \mu \mathrm{m}$. Remarkably, this behavior is quantitatively predicted in the calculation of the strain rate $\dot{\varepsilon}=\partial \mathrm{u} / \partial x$ (solid line in Figure 7-9b) that is calculated from the simulated velocity field given in Figure 7-7 (top). This indicates that the strain rate, which describes mechanical effects on the material under flow, can accurately describe the phenomena of supramolecular alignment within microdevices.

The extensive alignment of PPI 4/DNA assemblies in the region of high strain rate enables the visualization of further details regarding the $2 \mathrm{D}$ columnar lattice. At $x=300 \mu \mathrm{m}$ and $400 \mu \mathrm{m}$, two additional local maxima at $\chi \approx 0^{\circ}$ and $180^{\circ}$ can be found (red arrows in Figure 7-8b). This additional weak orientation perpendicular to the flow direction provides interesting insights into the response of a 2D phase of long chain DNA molecules to external stress. The majority of PPI 4/DNA microdomains are oriented parallel to the flow direction. In the region of maximum strain rate however, a concomitant orientation of some microdomains perpendicular to the flow direction occurs. This is most likely due to structural restrictions imposed by the long DNA strands and the square symmetry of the mesophase (schematically represented in Figure 7-10). The observed decrease in the intensity for $x \geq 1000 \mu \mathrm{m}$ is puzzling. Two phenomena are assumed to account for this decrease: firstly, a disintegration of formed complexes might have taken place owing to the strong increase in the dendrimer concentration, and, secondly, experimental inadequacies might have resulted in the fact that the X-ray beam did not fully hit the hydrodynamic focused DNA jet any more.

\subsubsection{Resolving initial steps of mesophase formation}

In Figure 7-8, the peak at $x=0 \mu \mathrm{m}$ corresponds to initial stages of DNA compaction and shows an asymmetrical profile. Owing to the absence of higher order peaks, the detailed structure cannot be ruled out. Accordingly, initial stages of DNA compaction leading up to the charge neutral or isoelectric point characterized by $N / P \approx 1.8$ $(\mathrm{pH}=8.5)^{53}$ are conducted by increasing the velocity of the DNA solution to $u_{\mathrm{DNA}}=200 \mu \mathrm{m}^{-1}$ and simultaneously reducing dendrimer solution velocities to $u_{\text {PPI }}=3 / 2 u_{\text {DNA }}$. Access to this regime is significant because the assembly of DNA containing mesophases varies as a function of the overall charge ratio, conforming to a physical description of charge inversion. ${ }^{211}$

$\mathrm{X}$-ray scans along the $x$ - as well as the $y$-direction within the microchannel device are shown in Figure 7-11. Owing to flow properties in microchannel devices, analyzing the compaction along $x$ - and $y$-direction correspond to each other. However, along the $y$ - 

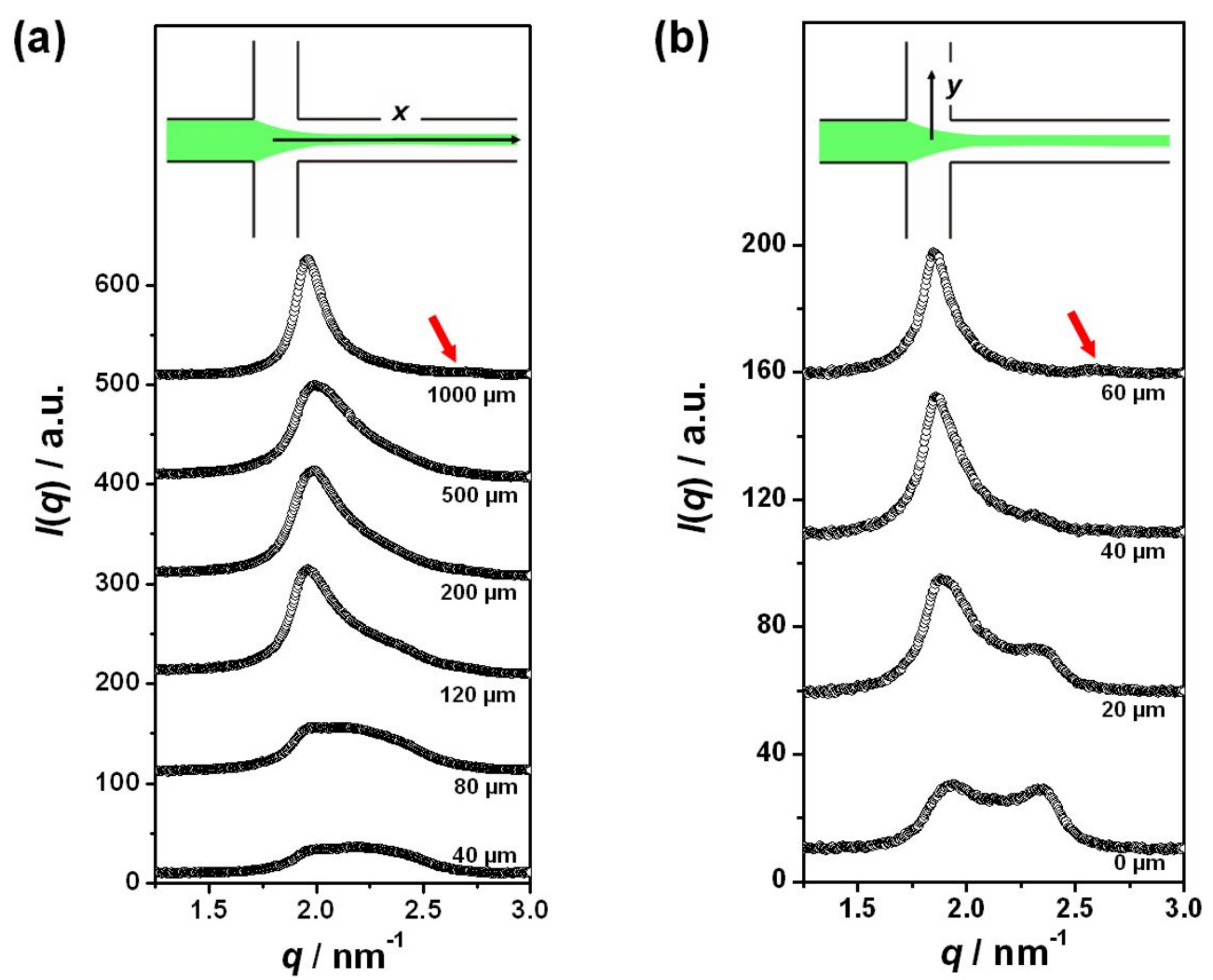

Figure 7-11: SAXS profiles of the early stages of PPI 4 mediated DNA compaction obtained at different $x$ positions along the main channel $(y=0)(a)$, and at different $y$ positions perpendicular to the main channel $(x=0)(b)$. The presence of a peak at $q_{1}$ is annotated by red arrows. ${ }^{209}$

direction the evolution of DNA compaction takes place on a compressed length scale. At the center of the confluence of the microchannels $(x=y=0)$, corresponding to minute dendrimer concentrations, X-ray pattern appear to be a convolution of multiple peaks with limiting peak positions $q_{0 \mathrm{a}} \approx 2.0 \mathrm{~nm}^{-1}$ and $q_{0 \mathrm{~b}} \approx 2.3 \mathrm{~nm}^{-1}$. Moving to higher $x$ or $y$ values (Figure 7-11a and $\mathrm{b}$, respectively), and therefore to increased dendrimer concentrations, the peak at $q_{0 \mathrm{a}}$ becomes dominant and features around $q_{0 \mathrm{~b}}$ disappear. Additionally, the peak position $q_{0 \mathrm{a}}$ is slightly shifted to smaller $q$ values. Eventually, a second peak $q_{1}=\sqrt{2} q_{0 \text { a }}$ (annotated by red arrows in Figure 7-11) is recorded, which refers again to a mesoscopic columnar phase with $2 \mathrm{D}$ in-plane square structure. This is surprising given that all measurement points are below the charge neutral point, where there is an excess of negatively charged DNA relative to the amount of dendrimers.

The appearance of $q_{0}$ at low dendrimer concentrations - and therefore a surplus of DNA - can be explained by a coexistence of two types of domains: firstly, positively charged domains with an enrichment of dendrimers, and secondly, negatively charged 

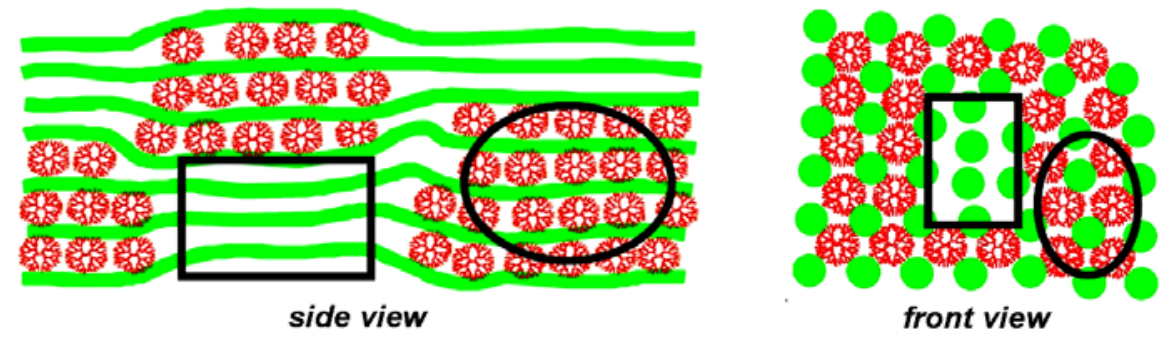

front view

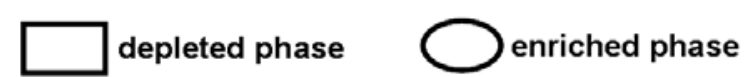

Figure 7-12: Schematic representation of the two coexisting phases in the low dendrimer regime: dendrimer-enriched (circle) and dendrimer-depleted (square). Side view (top) is along the microchannel, and front view (bottom) is a cross-section through the microchannel.

domains with a depletion of dendrimers. This is illustrated in Figure 7-12 from the side (along channel) and front (channel cross-section). Dendrimer-enriched domains show a columnar ordering of DNA molecules with intercalated dendrimers, having a spacing $d_{0 \mathrm{a}} \approx 3.2 \mathrm{~nm}$ (i.e. the square phase described previously). However, dendrimer-depleted domains show an ordering of DNA with a spacing that can be calculated from the peak position $q_{0 \mathrm{~b}} \approx 2.3 \mathrm{~nm}^{-1}$ and that is dependent on the lattice used to describe the mesophase. The diffraction peak at position $q_{0 \mathrm{~b}}$ is proposed to be due to a DNA columnar mesophase with a spacing $d \approx 2.7 \mathrm{~nm}$ (determined by $d=2 \pi / q$ ), which indicates a very dense DNA packing since it approaches the $2.5 \mathrm{~nm}$ diameter of hydrated DNA molecules. A similar peak at low $N / P$ values was reported by Evans et al., ${ }^{53}$ in which case its striking resemblance to that of hexagonal small cation-induced DNA bundles was noted. In this scenario, the lattice vector $a_{\mathrm{H}}=4 \pi / \sqrt{3} q \approx 3.2 \mathrm{~nm}$ reflects a unit vector of a hexagonal lattice with an interstitial area that is much too small to accommodate a PPI 4 molecule (without requiring a significant molecular deformation).

Regardless of the specific choice of the DNA lattice, in either case dendrimer molecules do not directly bridge DNA strands. Dendrimer bridging of DNA strands can be eliminated due to steric constraints: interstitial positions available to dendrimers in either a square or hexagonal DNA lattice are too small to accommodate PPI 4 molecules. Dendrimers nevertheless play an important role in the assembly of a onecomponent DNA mesophase, as is confirmed by experiments done on DNA solutions without dendrimers that show qualitatively different X-ray patterns (chapter 5.2.1 and 5.3.3). 


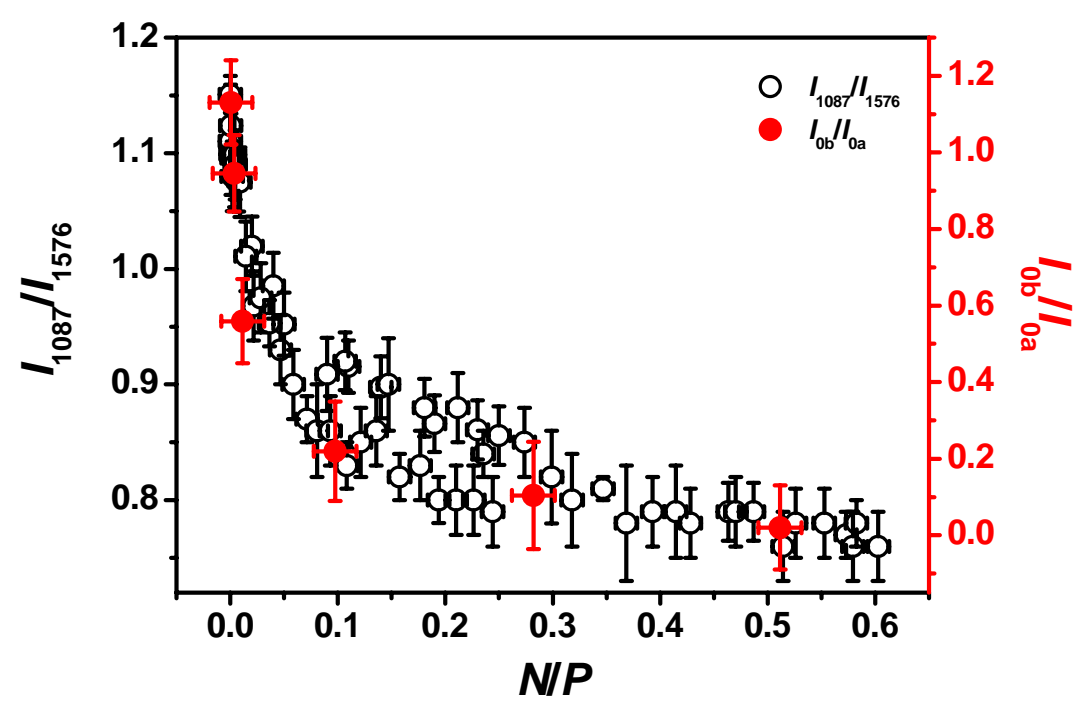

Figure 7-13: $N / P$-dependence of the intensity ratio $I_{1087} / I_{1576}$ obtained from Raman measurements and $I_{0 \mathrm{~b}} / I_{0 \mathrm{a}}$ obtained from X-ray microdiffraction experiments.

The most reasonable model, by process of elimination, is one including domains rich and poor in dendrimers. This is reminiscent of a scenario reported using DNA and surfactants. ${ }^{212}$ The close packing of DNA to length scales $d \approx 2.7 \mathrm{~nm}$ known to occur in the presence of multivalent salts is considered to be a result of a balance of forces acting to resist (e.g. bending, mixing entropy) or favor (e.g. counter-ion fluctuations, hydration) condensation. ${ }^{213}$ For the presented measurements, no additional salts have been explicitly added but a balance of similar forces is likely at play. The two-phase dendrimer/DNA model is corroborated by a vanishing of dendrimer-depleted domains $\left(q_{0 \mathrm{a}} \leq q \leq q_{0 \mathrm{~b}}\right)$ with a concurrent domination of square dendrimer/DNA mesophases at increasing values.

\subsubsection{Comparison with confocal Raman measurements}

The evolution of the non-equilibrium structure formation can be monitored in terms of the intensity ratio $I_{0 \mathrm{~b}} / I_{0 \mathrm{a}}$ of the two Bragg reflections at $q_{0 \mathrm{~b}}$ and $q_{0 \mathrm{a}}$. Observed X-ray pattern can be interpreted in terms of a structural transition of PPI 4/DNA complexes from dendrimer depleted microdomains to dendrimer enriched microdomains with square in-plane symmetry. A coexistence regime of the two phases is observed for positions $x<1000 \mu \mathrm{m}$. In order to correlate structural information from X-ray microdiffraction experiments to the $N / P$ ratio, finite element simulations are performed 
according to flow conditions used in chapter 7.2.2 in order to resolve initial steps of mesophase formation.

In Figure 7-13, the ratio of intensity $I_{0 \mathrm{~b}} / I_{0 \mathrm{a}}$ of the X-ray diffraction peaks at $q_{0 \mathrm{a}}$ and $q_{0 \mathrm{~b}}$ is plotted versus $N / P$ and contrasted to the ratio of intensity $I_{1087} / I_{1576}$ obtained from confocal Raman measurements (Figure 7-6). Comparing the two data sets shows that the vanishing of dendrimer-depleted domains with increasing $N / P$ is associated with an increased complexation of negatively charged phosphate groups by dendrimeric amine groups. On a molecular level, the onset of the square columnar mesophase seems to correspond to a complete complexation of all (accessible) DNA phosphate groups by dendrimeric amine sites at $N / P \approx 0.55$.

\subsection{DNA compaction by dendrimers of intermediate size and charge - a generalized description}

The dynamic assembly of DNA compaction induced by PPI 4 dendrimers has been studied in great detail in chapter 7.1 and 7.2 showing PPI 4/DNA mesophase formation. Utilizing hydrodynamic focusing devices, it is possible to access - for the first time in a controlled manner - very low $N / P$ ratios. Surprisingly, in such regions of excess DNA a coexistence of densely packed mesophases containing DNA only and mesophases consisting of PPI 4 and DNA is observable. The question arises in how far this unexpected structure formation is only specific to the PPI 4/DNA interaction and to what extend it represents a general feature of the interaction between dendrimers of an intermediate size and charge and DNA.

To address this issue, X-ray microdiffraction measurements of DNA compaction induced by two additional dendrimers of intermediate size and charge, namely PPI 3 and PAMAM 3, are performed. According to results presented in chapter 6, PAMAM 3 dendrimers possess a similar charge and size as PPI 4 dendrimers, whereas PPI 3 dendrimers are significantly smaller and possess only half of the charge of PPI 4. Representative X-ray diffraction patterns of PAMAM 3 and PPI 3 induced DNA condensation are shown in Figure 7-14 and Figure 7-15, respectively. The scattering intensity is plotted in terms of the reciprocal vector $q$. Plots of X-ray data in the figures are offset for clarity.

2D columnar mesophases of PAMAM 3/DNA are identified for different charge ratios using microfocused X-ray diffraction. For small $N / P$ ratios, a single broad peak at a position $q_{0} \approx 1.8 \mathrm{~nm}^{-1}$ is observed. This corresponds to the regime of excess DNA. At 

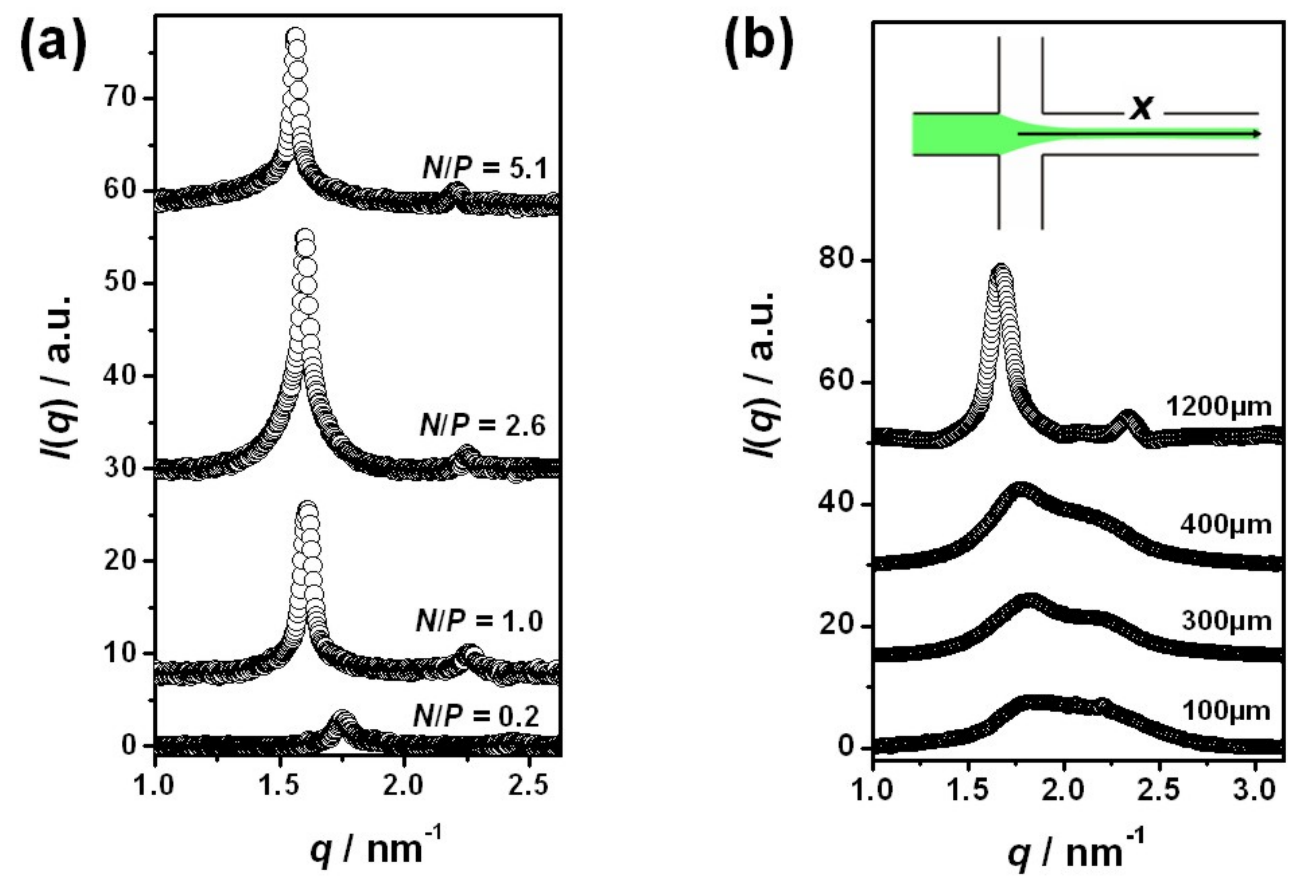

Figure 7-14: (a) SAXS profiles of PAMAM 3/DNA mesophases at different $N / P$ ratios. (b) SAXS profiles of initial stages of PAMAM 3 mediated DNA compaction at different $x$-positions along the outlet channel.

higher $N / P$ ratios, the $q_{0}$-peak is shifted towards smaller $q$ values and becomes more prominent. A second peak $q_{1}$ is concurrently arising. Analog to PPI 4/DNA mesophases, the relation $q_{1}=\sqrt{2} q_{0}$ of the two peak positions is consistent with a columnar mesophase with in-plane square symmetry. An increase in the lattice spacing $d_{\text {PAMAM3 }}$ is obtained with increasing $N / P$ ratio. According to the discussion in chapter 7.2.1, this can be understood by the incorporation of additional dendrimer molecules within mesophases.

Utilizing a hydrodynamic focusing microflow device enables to pinpoint reaction dynamics at low charge ratios below the charge neutral point, where DNA is in excess of dendrimers. A $5 \mathrm{mg}^{-1} \mathrm{~mL}^{-1}$ DNA solution is injected in the main channel with a mean velocity of $u_{\mathrm{DNA}}=300 \mu \mathrm{ms}^{-1}$ and $10 \mathrm{mg} \mathrm{mL}^{-1}$ PAMAM 3 dendrimer solutions are added to the side channels with a mean velocity of $u_{\text {PAMAM } 3}=2 u_{\text {DNA }}$. Figure $7-14 \mathrm{~b}$ shows the evolution of PAMAM 3/DNA mesophase formation at different positions along the reaction channel corresponding to different $N / P$ ratios. Mesophase formation analog to the one observed for PPI 4 induced DNA compaction is found. X-ray patterns again appear to be a convolution of multiple peaks. The limiting peak positions are $q_{0 \mathrm{a}} \approx 1.8 \mathrm{~nm}^{-1}$ and $q_{0 \mathrm{~b}} \approx 2.3 \mathrm{~nm}^{-1}$. According to findings reported for the PPI 4/DNA structure formation (chapter 7.2.2), increasing the PAMAM 3 dendrimer concentration 

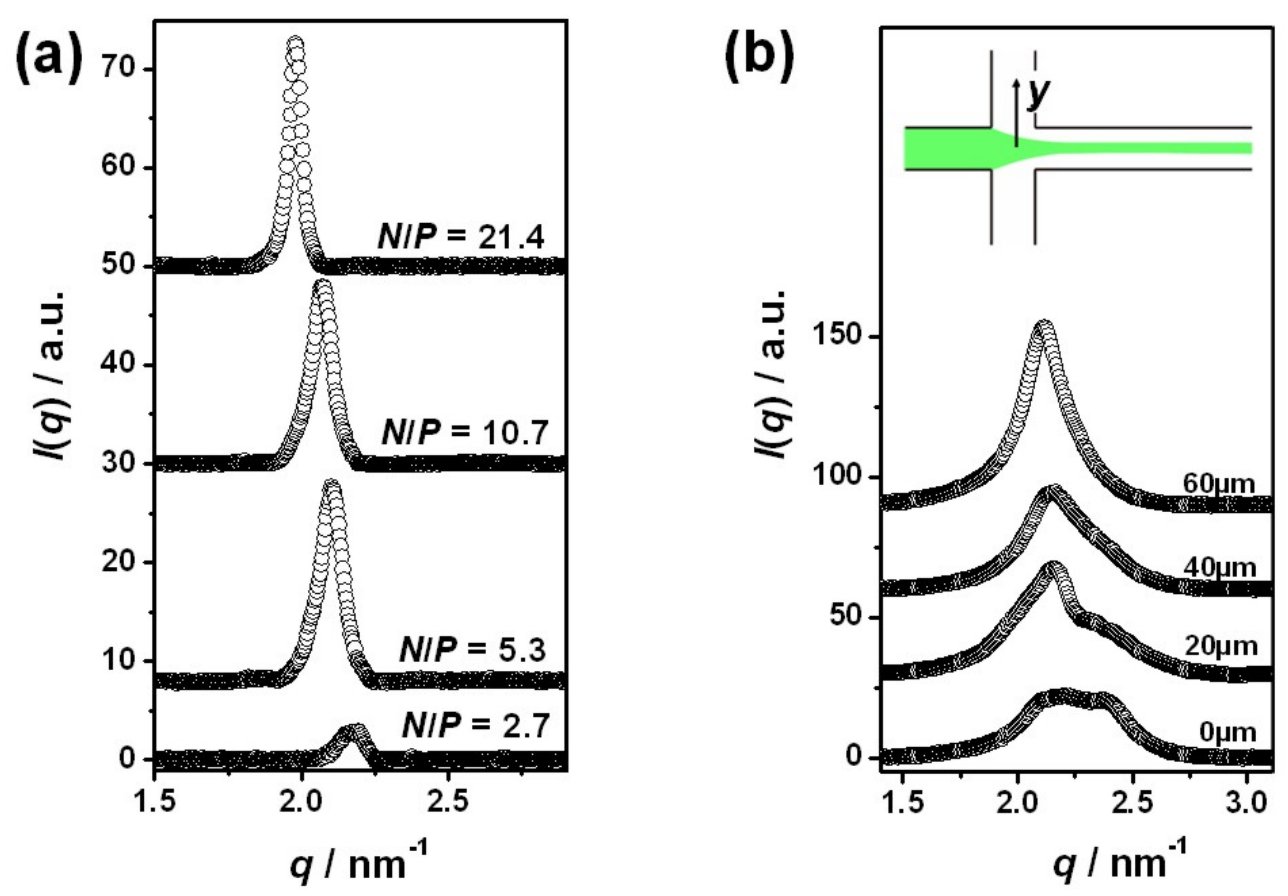

Figure 7-15: (a) SAXS profiles of PPI 3/DNA mesophases at different $N / P$ ratios. (b) SAXS profiles of the initial stages of PPI 3 mediated DNA compaction at different $y$-positions perpendicular to the reaction channel.

results in a more dominant peak at $q_{0 \mathrm{a}}$ associated with a disappearance of features around $q_{0 \mathrm{~b}}$. Additionally, the peak position $q_{0 \mathrm{a}}$ is slightly shifted to smaller $q$ values. The second peak at $q_{1}=\sqrt{2} q_{0 \text { a }}$ refers again to a mesoscopic columnar phase with a $2 \mathrm{D}$ in-plane square symmetry.

Figure 7-15a shows SAXS profiles obtained from PPI 3/DNA mesophases at different $N / P$ ratios. A single peak at a position $q_{0} \approx 2.2 \mathrm{~nm}^{-1}$ is observed, which is shifted towards smaller $q$ values and becomes more prominent with increasing $N / P$. As known from literature, ${ }^{53,214} \mathrm{X}$-ray diffraction patterns of PPI 3/DNA mesophases are strikingly similar in $q$ position and profile to the peak seen with polyamines spermine and spermidine complexing DNA. Accordingly, low-molecular dendrimers with only a few cationic groups and fragmentarily developed architecture are assumed to compact DNA similarly to such conventional polyamines. Polyamine/DNA complexes exhibit a hexatic structure with lattice constant of e.g. $a_{H}=4 \pi / \sqrt{3} q_{0}=3.0 \mathrm{~nm}$ for spermidine/DNA. ${ }^{215}$ PPI 3/DNA complexes are likely to have the same structure giving a real space DNA-DNA distance of approximately $3.1 \mathrm{~nm}$. However, in the absence of well defined higher order diffraction peaks, it cannot be ruled out a distorted hexagonal structure. 
The formation of a mesophase with hexatic in-plane symmetry indicates a change in the DNA compaction mechanism from an electrostatically driven, partial adsorption of DNA chains on the oppositely charged dendrimer nanospheres, to a like-charge attraction, where low generation dendrimer molecules play the role of environmental parameters. ${ }^{10}$ However, X-ray diffraction measurements in a hydrodynamic focusing device $\left(u_{\mathrm{DNA}}=500 \mu \mathrm{m}^{-1}, \quad u_{\mathrm{PPI} 3}=600 \mu \mathrm{m}^{-1}, \quad c_{\mathrm{DNA}}=5 \mathrm{mg} \cdot \mathrm{mL}^{-1}, \quad c_{\mathrm{PPI} 3}=10 \mathrm{mg} \cdot \mathrm{mL}^{-1}\right)$ performed in the low $N / P$ regime show qualitatively the same evolution of a two-phase dendrimer/DNA complex structure as has been observed for PPI 4 and PAMAM 3 dendrimers. SAXS profiles given in Figure 7-15 unravel a convolution of multiple peaks with limiting positions $q_{0 \mathrm{a}} \approx 2.1 \mathrm{~nm}^{-1}$ and $q_{0 \mathrm{~b}} \approx 2.4 \mathrm{~nm}^{-1}$ (corresponding to $d_{0 \mathrm{a}} \approx 3.0 \mathrm{~nm}$ and $\left.d_{0 \mathrm{~b}} \approx 2.6 \mathrm{~nm}\right)$. With increasing $N / P$, features around $q_{0 \mathrm{~b}}$, corresponding to dendrimer depleted domains, disappear and a concurrent domination of the dendrimer enriched domains around $q_{0 \mathrm{a}}$ is observable.

The results obtained from PPI 3, PPI 4, and PAMAM 3 induced DNA compaction suggest that the observed compaction mechanism characterized by a coexistence of dendrimer depleted and dendrimer enriched domains represents a general feature of the interaction between dendrimers of an intermediate size and charge and DNA. Moreover, data obtained from PPI 3/DNA complexes indicate that this alternate route of DNA compaction in the low $N / P$ region should actually be observable when studying DNA compaction mediated by conventional polyamines.

\subsection{Conclusions}

In summary, the potent combination of microfluidics with confocal Raman microscopy and small-angle X-ray microdiffraction allows for detailed insights into the evolution of dendrimer induced DNA compaction. The diffusive mixing in microchannels provides tunable reaction conditions with defined changes in local concentrations. This allows for acquiring of experimental data at any desired $N / P$ ratio. A major advantage of the experimental setup is the acquisition of multiple $N / P$ data points on a single device while using extremely small amounts of material without concern (due to the continuous flow of materials) for radiation damage.

Employing chemically sensitive confocal Raman microscopy, molecular interactions of DNA and dendrimers are studied. The fact that the negatively charged phosphate groups along the DNA backbone are found to be the main targets of dendrimer amine groups reflects the electrostatic nature of the dendrimer induced DNA compaction. Furthermore, combining high resolution 2D confocal Raman data and finite element 
simulations indicates that experimental parameters employed here are such that the diffusion time scale is much larger than the reaction time scale.

2D columnar mesophases are identified for different $N / P$ ratios using microfocused $\mathrm{X}$ ray diffraction. The mesophase lattice spacing $d$ and the degree of orientation $\Delta \chi$ correspond to values of $N / P$ and the strain rate $\dot{\varepsilon}$, respectively, both of which are calculated using finite element simulations. An added benefit of the experimental setup is the resulting alignment of mesophases transverse to the flow direction in the microdevice.

Moreover, for the first time, access to very low $N / P$ ratios in a controlled manner is enabled, where a coexistence of DNA/DNA and dendrimer/DNA interactions appears to occur. Generally, DNA is considered to condense in biological systems via multivalent salts or proteins. ${ }^{1,8,193,211,216}$ Most surprising, our results indicate that in an undercharged (low $N / P$ ) regime it is possible that DNA condensation occurs without direct contact to such materials. Comparing Raman and X-ray data shows that the vanishing of dendrimer-depleted domains with increasing $N / P$ is associated with an increased complexation of negatively charged phosphate groups by dendrimer amino groups. The complexation of a maximum amount of (accessible) phosphate groups is corresponding to the (full) emergence of the columnar mesophase with the in-plane square symmetry at $N / P \approx 0.55$.

The consistency of X-ray data obtained from DNA complexes with three different types of dendrimers suggests that the observed compaction mechanism at low $N / P$ ratios represents a more general feature of DNA compaction. This mechanism may also exist in biological cells, which are dense and contain a variety of surrounding protein aggregates. In addition, the stages of DNA packing and ejection in bacteriophage are a subject of considerable biophysical interest and involves a dense packing of DNA suggestive of the assembly found here for low $N / P$ values. ${ }^{217}$ 


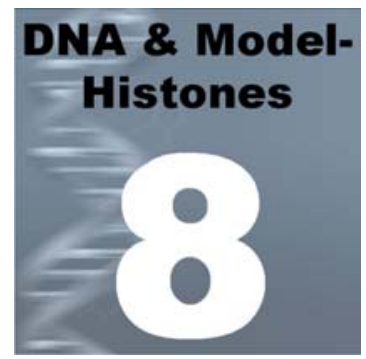

\section{DNA COMPACTION: Do DENDRIMERS MIMIC HISTONES?}

DNA compaction in vivo has to account for a multitude of different task. Hence, the structure of chromatin is very sophisticated and still widely unknown. ${ }^{10,}{ }^{20} \mathrm{Up}$ to now, the architecture of the $30 \mathrm{~nm}$ fiber and the higher order assemblies of chromatin have not been elucidated, nor have the forces that drive their formation been identified. In order to unravel the structure of chromatin, several researchers have performed in vitro studies of nucleosome core particles (NCP) organized in crystals and mesophases. ${ }^{22,218-220}$ In particular due to the fact that supramolecular organization in dense assemblies is the result of a tight packaging of particles into a regular arrangement driven only by the search for an energetically favorable mode of interaction between neighboring particles, it is widely believed that crystal packing may reflect some important aspects of inter-nucleosome interaction in vivo. ${ }^{218,}$ 220-222 However, the most obvious difference between chromatin on the one hand and NCP crystals or mesophases on the other hand is the fact that all individual nucleosomes are connected in chromatin by the linker-DNA, while they exist as individual, unconnected particles in NCP crystals and mesophases.

In this chapter PAMAM dendrimers generation 6 with dimensions and charges well comparable to those of the histone core are used to compact DNA. Owing to the significant higher charge and size of PAMAM 6 compared to dendrimers of lower generations, a changed organization of PAMAM 6/DNA complexes is expected including in particular local wrapping of DNA around the dendrimers. However, a direct prove of such a wrapping scenario is still missing. In particular, comparing structural details of the organization of PAMAM 6/DNA and NCPs may help to retain 
some important characteristics of the multiple interactions and possible supramolecular organizations in chromatin. It is important to point out that the PAMAM 6/DNA system with no added salt represents the model system of lowest possible complexity (only artificial "proteins", only monovalent ions, no additionally added salt, no other organic compounds) that can possibly be employed to mimic DNA/histone interactions. From a more fundamental point of view, macroion/polyelectrolyte complexes are a common pattern in chemistry, physics, and biology. ${ }^{223,224}$ According to their high importance, there are a significant number of theoretical studies on the topic reported in literature. $^{7,9,10}$ However, the number of experimental papers cited by theoretical physicists is limited to only a few experimental studies. Therefore, experimental results on PAMAM 6/DNA interactions are expected to be an important contribution to the field. Moreover, dendrimer/DNA complexes are currently extensively used for DNA transfection. ${ }^{162,225,226}$ To clarify fundamental aspects of involved transfection mechanisms, e.g. the observed generation dependence of transfection efficiency or the varying sensitivity of incorporated DNA to nuclease digestion, it is essential to investigate the structure of dendrimer/DNA complexes at a molecular level and to obtain new insights into formed supra-molecular structures. This may also serve to improve the design of new non-viral gene transfection vectors.

\subsection{Initial states of PAMAM 6/DNA complex formation}

In a first attempt to access the PAMAM 6/DNA complex formation, a set of synchrotron X-ray microdiffraction experiments are performed in flow using a hydrodynamic focusing device with a channel depth of $300 \mu \mathrm{m}$ and a width of $150 \mu \mathrm{m}$. A semi-diluted aqueous DNA solution with a concentration of $c_{\mathrm{DNA}}=7.5 \mathrm{mg}^{-1} \mathrm{~mL}^{-1}$ is injected in the main channel with a mean flow velocity of $u_{\mathrm{DNA}}=0.009 \mu \mathrm{m}^{\mathrm{s}} \mathrm{s}^{-1}$. The DNA stream is initially hydrodynamically focused by injecting pure water from the side channels with a velocity of $u_{\text {side }}=2.5 u_{\text {DNA }}$. After establishing a stationary flow, PAMAM 6 dendrimers are continuously added to both side streams. Dendrimer concentrations in the side channels are adjusted to $35 \mathrm{mg} \mathrm{mL}^{-1}$. The lamellar flow conditions inside the microchannels allow for a purely diffusive mixing of components resulting in a final charge ratio of $N / P \approx 11$ after complete mixing. All solutions are adjusted to $\mathrm{pH}=8.5$. According to chapter 6.2 , at this $\mathrm{pH}$ about $50 \%$ of all primary 
(a)

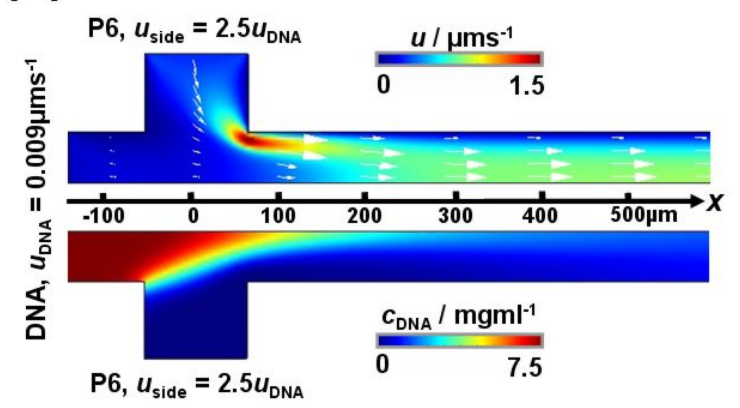

(b)

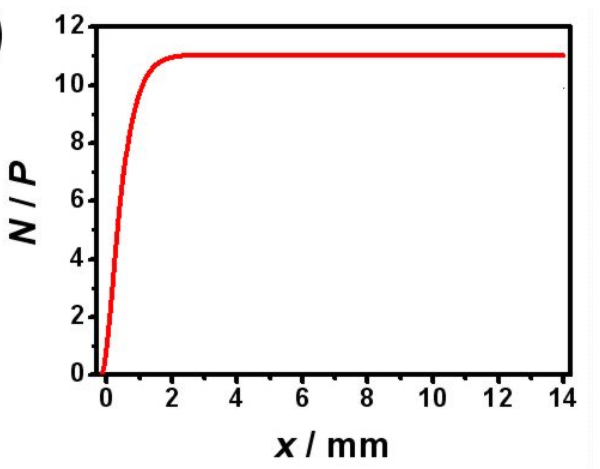

Figure 8-1: (a) Finite element simulations showing the flow field (upper part) and the DNA concentration distribution (lower part). (b) $x$-dependence of local $N / P$ ratio at a position in the middle of the outlet channel $(y=0)$.

amino groups are charged, whereas all tertiary are uncharged. The total charge of the molecule is about $150 \mathrm{e}^{+}$whereas the radius of the dendrimer is $R_{\mathrm{P} 6}(\mathrm{pH}=8.5)=3.0 \mathrm{~nm}$.

\subsubsection{Finite element simulations}

The physical conditions inside the microchannels are modeled by finite element simulations of the experimental situation (chapter 2.3). A detailed comparison of the simulation and the experiment allows for an improved understanding. According to simulations and results presented in chapter 5.3 and 7.1, the diffusion constant of PAMAM $6, D_{\mathrm{P} 6}$, and the viscosity of formed PAMAM 6/DNA assemblies, $\eta_{\text {complex }}$, are the only unknown parameters in the simulation. Due to reasons discussed in what follows, imaging of PAMAM 6/DNA complex formation in microflow was impossible as hence was an experimental determination of these two parameters. However, results obtained from analyzing dendrimer/DNA complexes in microflow, show that the viscosity of the formed DNA complexes is about three orders of magnitude higher than the viscosity of water, $\eta_{\text {complex }} \approx 10^{3} \eta_{\text {water }}$. This value seems to be largely independent of the exact nature of compaction agents. ${ }^{59,104}$ Moreover, obtained values for the diffusion constants of all compaction agents are close to values obtained from the Stokes-Einstein relation in water. Accordingly, simulations are performed using $\eta_{\text {complex }}=10^{3} \eta_{\text {water }}$ and $D_{\mathrm{P} 6}=7.5 \cdot 10^{-11} \mathrm{~m}^{2} \mathrm{~s}^{-1}$. Obtained results are presented in Figure 8-1a showing the flow field (top) and the local DNA concentration (bottom). Figure 8-1b gives the $x$-dependence of the local $N / P$ ratio at a position $y=0$ in the middle of the outlet channel. 


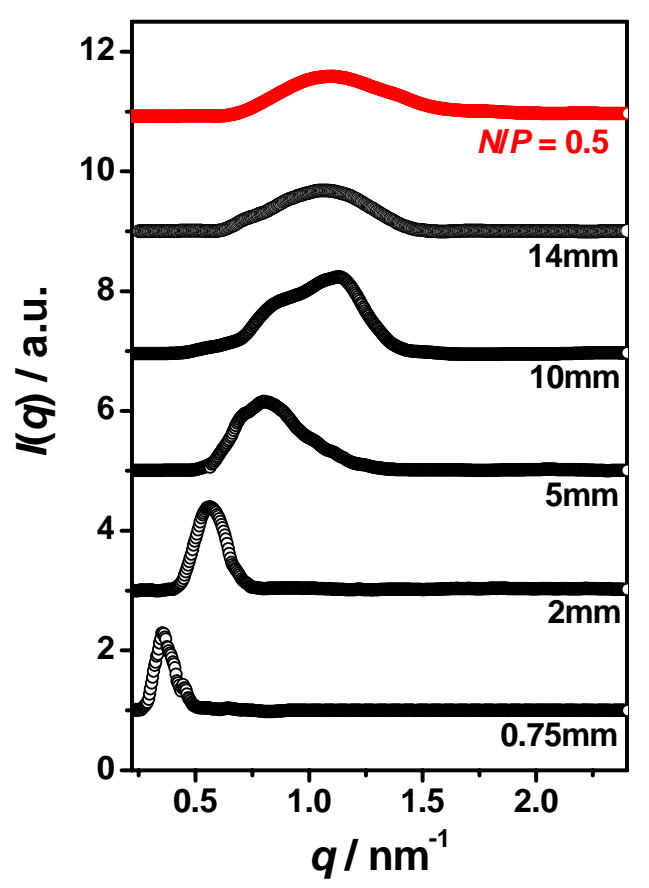

Figure 8-2: $X$-ray diffraction patterns obtained in flow at different positions $x$ in the middle of the outlet channel $(y=0)$. Data are contrasted to the diffraction pattern obtained from a bulk sample of $N / P=0.5$ (red).

\subsubsection{X-ray microdiffraction measurements in flow}

Subsequently to the channel intersection, the interaction of dendrimers with DNA can be monitored along the outlet channel. A lateral scanning of the sample allows for spatially resolved small angle X-ray diffraction experiments along the microfluidic channels. The positional accuracy of absolute coordinates in the microdevice is on the order of the beam size $(20 \mu \mathrm{m})$. CCD images are collected with exposure times of 30-120s per position. Representative X-ray diffraction patterns of PAMAM 6 induced DNA condensation measured at different distances $x$ from the confluence center are plotted in terms of the reciprocal vector $q$ and shown in Figure 8-2. Plots of the X-ray data in the figure are offset for clarity.

$\mathrm{X}$-ray pattern are composed of several overlapping peaks indicating a coexistence of a multitude of different microdomain structures. Figure 8-3 demonstrates that the decomposition is successfully made by assuming Lorentzian peak shapes. Best fitting the data yields corresponding peak positions $q$ and correlation lengths $L_{\mathrm{C}}=2 \pi / \Delta q$. In Figure 8-4, the dependence of the peak positions $q$ on the position $x$ along the outlet channel is given. A progressive shift in peak position starting from $q \approx 0.35 \mathrm{~nm}^{-1}$ 

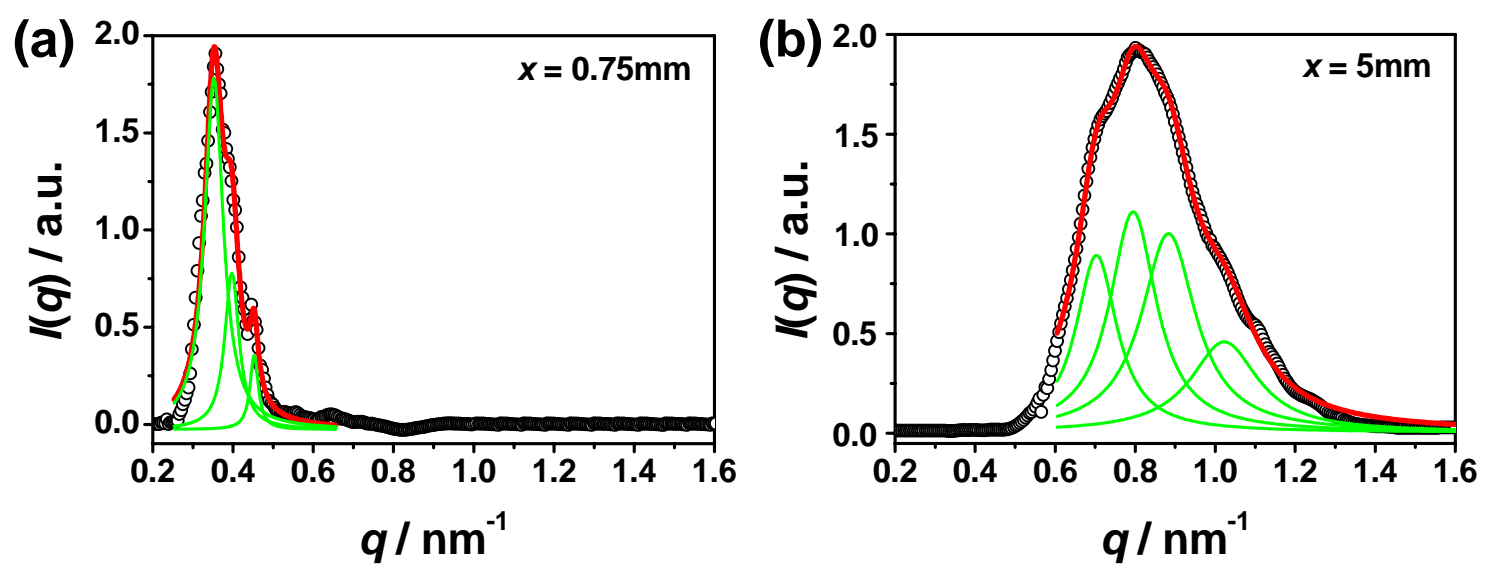

Figure 8-3: Decomposition of X-ray diffraction patterns by fitting with Lorentzian functions demonstrated for two representative data sets.

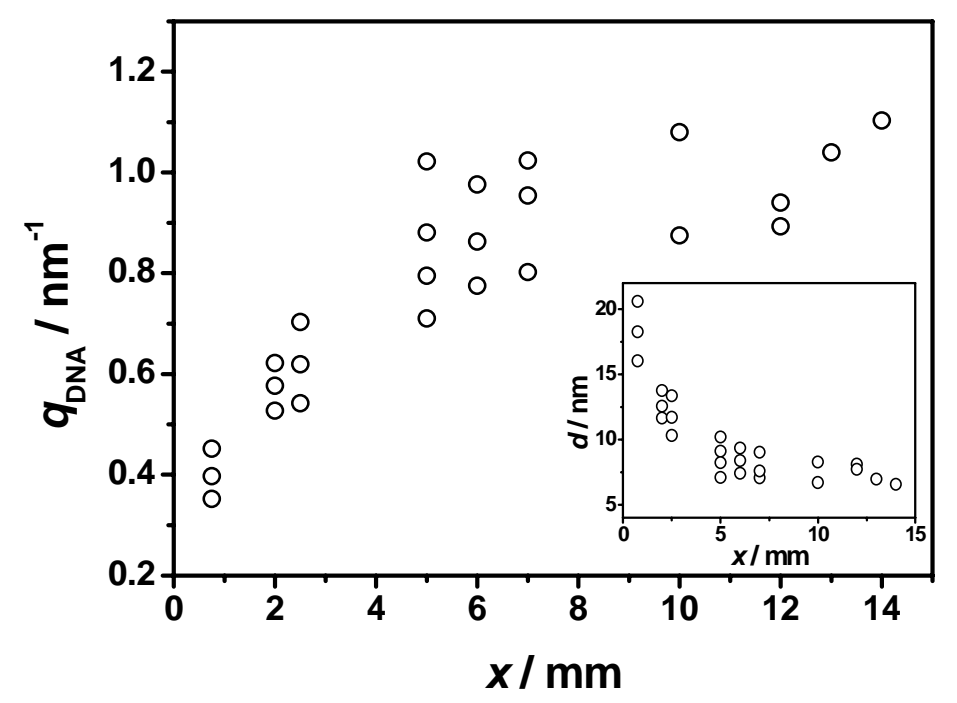

Figure 8-4: Dependence of the peak position $q$ on the position $x$ along the outlet channel. The inset picture gives the $x$-dependence of $d=2 \pi / q$.

towards higher $q$ values is observed. This increase in $q$ corresponds to shorter DNADNA distances, $d$. For $x>12 \mathrm{~mm}, \mathrm{X}$-ray profiles exhibit only a single peak indicating that all PAMAM 6/DNA microdomains are of the same structure. From the large variety of structures observed for PAMAM 6/DNA complexes, it seems reasonable to conclude that the apparent limitation of structural types observed in bulk samples is in reality a reflection of the experimental difficulty in the characterization of less stable intermediate phases of a shorter range order.

Figure 8-5a gives the dependence of the correlation length $L_{\mathrm{C}}$ on the position $x$ along the outlet channel. Starting from $L_{\mathrm{C}} \approx 110 \mathrm{~nm}$, the correlation length is significantly 

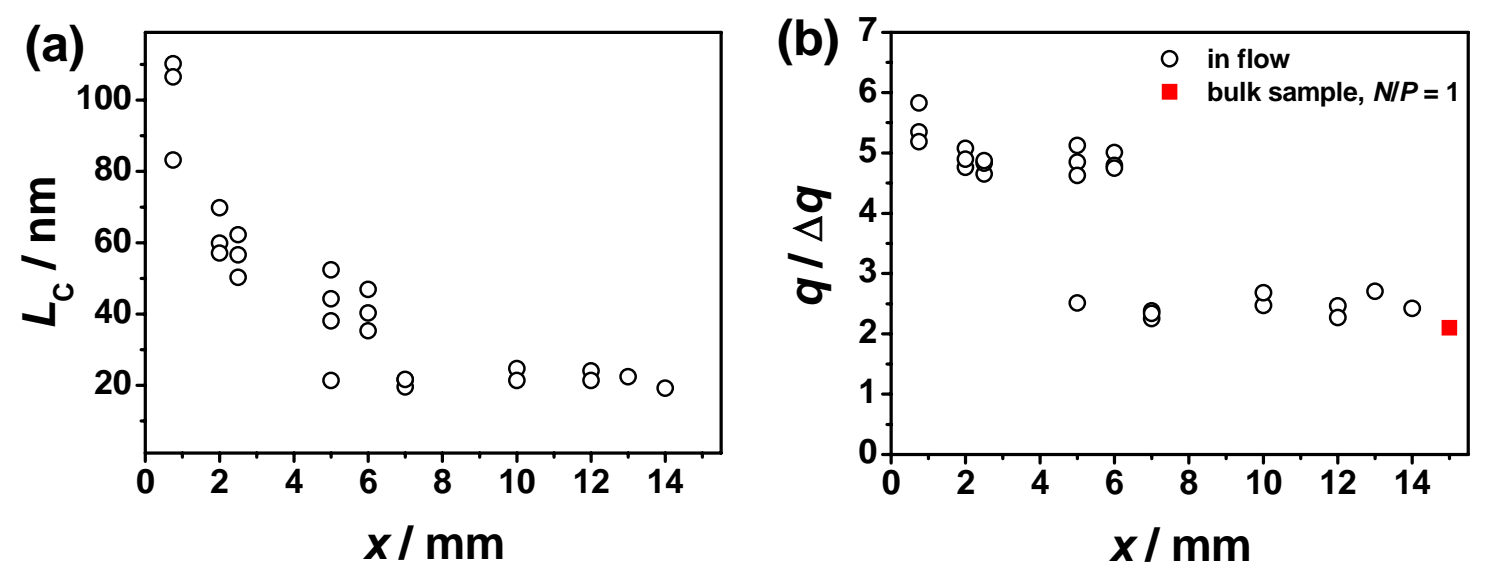

Figure 8-5: (a) Dependence of the correlation length $L_{\mathrm{c}}$ on the position $x$ along the outlet channel. (b) Dependence of the correlation length in terms of DNA repeats, $q / \Delta q$, on $x$. For comparison, the value obtained from bulk measurements $(N / P=0.5)$ is given.

reduced with increasing $x$ to $L_{\mathrm{C}} \approx 19 \mathrm{~nm}$. In order to account for comparable data, Figure $8-5$ b shows the $x$-dependence of the correlation length given in terms of DNA repeats, $L_{\mathrm{C}} / d=q / \Delta q$. Although the correlation length is significantly reduced with changing the observation position, the degree of correlational order seems to stay relatively constant at $q / \Delta q \approx 5$. Strikingly, this implies that although the correlation length is depending on the lattice spacing, the degree of correlational order is not. At $x \approx 4 \mathrm{~mm}$, microdomains with a significantly reduced degree of correlational order of $q / \Delta q \approx 2.4$ emerge. Moving the observation position further down the outlet channel, a transition takes place towards the less positionally ordered phase at $4<x<7 \mathrm{~mm}$. For $x \geq 7 \mathrm{~mm}$, only microdomains with the reduced positional order are observed.

In Figure 8-2, X-ray diffraction profiles obtained in flow are contrasted to profiles obtained from a PAMAM 6/DNA bulk sample at $N / P=0.5$ (red). At $N / P=0.5$, PAMAM 6/DNA complexes give rise to a single broad correlation peak at $q=1.12 \mathrm{~nm}^{-1}$. When analyzed with polarization optical microscopy, PAMAM 6/DNA complexes of this composition reveal an optically (weakly) birefringent pattern. This indicates that the DNA chains in the complex are orientationally ordered. It is hence reasonable to designate the observed mesomorphic structure at $N / P=0.5$ and the corresponding phases observed in flow as nematic liquid-crystalline. The packing lacks long-range positional order as the correlation peak is broad. From $q / \Delta q \approx 1.9$, it can be deduced that intermolecular positional ordering exists only between nearest neighbors. The positional ordering of the bulk sample is close to that observed in the microchannel at $x \geq 7 \mathrm{~mm}$. This can be readily seen in Figure $8-5 \mathrm{~b}$, where values of $q / \Delta q$ 
corresponding to the nematic phases observed in bulk measurements are given for comparison.

\subsubsection{Reaction limited structure formation}

In chapter 7, dendrimers generation 3 and 4 with an intermediate size $(3-4 \mathrm{~nm})$ that is conveniently between that of small polyamines (spermidine, spermine) and histone octamers are used to compact DNA. At low $N / P$ ratios, a coexistence of dendrimer enriched and dendrimer depleted domains has been observed. Although it is not possible to rule out the exact nature of PAMAM 6/DNA complexes formed in flow, observed X-ray patterns can not be described in terms of a coexistence of such domains.

Performing measurements in flow allows for time-resolved access of dendrimer/DNA interactions. Comparing experimental results with finite element simulations performed for dendrimers generation 3 and 4 shows that ongoing reaction processes are very fast and only limited by the dendrimer diffusion (chapter 7). Most notably, using the larger PAMAM 6 dendrimers this is no longer the case.

In Figure $8-1 \mathrm{~b}$, the $x$-dependence of the local $N / P$ ratio is given at $y=0$ showing that for positions $x>1.3 \mathrm{~mm}$, complete mixing of reactants is achieved. Accordingly, the final complex composition of $N / P \approx 11$ is maintained until the end of the outlet channel at $x=14 \mathrm{~mm}$. This distance corresponds to a time of $1210 \mathrm{~s}$. Contradictory to this fact, Figure 8-2 shows that the structural evolution of PAMAM 6/DNA complexes extends over the whole length of the outlet channel. Moreover, X-ray patterns observed at the furthest measurable position of the device, $x=14 \mathrm{~mm}$, are apparently rather corresponding to the one observed from bulk complexes with a composition of $N / P=0.5$.

One may speculate that a value according to the Stoke-Einstein relation overestimates $D_{\text {P6 }}$. However, it is not possible to establish a concentration gradient in the middle of the outlet channel, which extents over the whole length of the device, by varying the dendrimer diffusion constant within physically reasonable limits. This strongly indicates that, compared to the smaller dendrimers generation 3 and 4, the kinetics of phase transitions observed for PAMAM 6 and DNA along the outlet channel are particularly slow and not diffusion limited any more.

Studying PAMAM 6/DNA complex formation in microflow reveals multiple intermediate phases and a complex transition behavior with a coexistence of multiple phases. This strongly resembles the case of protein folding, where the condensation process also exhibits multistage dynamics. ${ }^{27}$ The multitude of different stages 
observed during the complex formation may directly reflect the fact that the long chain DNA molecules have to overcome several energetic and entropic bottlenecks in order to find their optimal organization on the dendrimer surface. This clearly indicates a fundamental change in the DNA compaction mechanism with increasing size of the compaction agent. In what follows, bulk measurements are employed in order to unravel the interaction mechanism of PAMAM 6/DNA.

\subsection{Unraveling the interaction mechanism}

\subsubsection{Form factor of PAMAM 6/DNA entities at $\mathrm{pH}=\mathbf{8 . 5}$}

In order to elucidate the nature of PAMAM 6/DNA interaction, the first point to determine is the shape and the dimensions of the scattering entities. Such information can be obtained by analyzing the corresponding form factor. For sufficiently diluted samples, the scattering intensity is considered to be free from any detectable interparticle effects (equation 6-3). SAXS measurements of such solutions may yield the form factor of the scattering entities (chapter 6.2). Therefore, a dilute sample with a solute mass fraction of $w / w \approx 0.05-0.1 \%$ and a complex composition of $N / P \approx 2$ has been analyzed at $\mathrm{pH}=8.5$. It is important to notice that a precipitation of complexes from solution can occur. This leads to a further reduction of the PAMAM 6/DNA mass fraction in the supernatant. Taken advantage of the fact that dendrimer/DNA complexes - contrary to protein/DNA complexes as discussed in chapter 9.4.3 - are insensitive to high dose X-ray irradiation, all measurements are taken at ambient temperature with exposure times of 3600-7200s. The 2D SAXS images are azimuthally averaged to produce $1 \mathrm{D}$ intensity profiles $I(q)$ for scattering vector values in the interval $\left[0.26 \mathrm{~nm}^{-1}, 2.08 \mathrm{~nm}^{-1}\right]$. In addition, the background from the pure solvent is also collected. After normalization of the scattering data, corresponding background data are subtracted. The result is shown in Figure 8-6.

In a first attempt to estimate the overall shape and size of PAMAM 6/DNA entities, the experimental data are fitted with the scattering intensity expected from objects of a simple geometry. The scattering entities have been approximated by assuming an overall spherical $(R=3.8 \mathrm{~nm})$, cylindrical $(R=3.8 \mathrm{~nm}, H=2.8 \mathrm{~nm})$, and an ellipsoidal ( $A=B=4.1 \mathrm{~nm}, C=3.0 \mathrm{~nm})$ shape, respectively. The dimensions of the model particles have been adjusted to yield best fits to the experimental data. Calculated X-ray patterns 

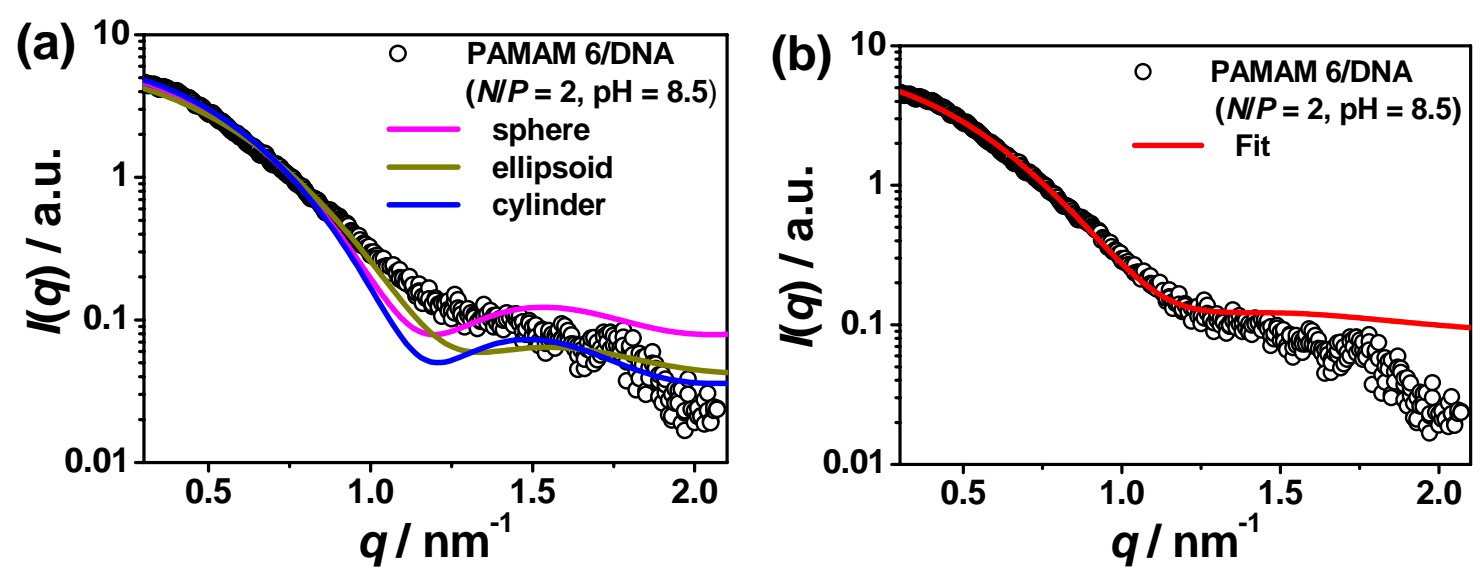

Figure 8-6: SAXS data obtained from a dilute PAMAM 6/DNA solution with a complex composition of $N / P \approx 2$ at $\mathrm{pH}=8.5$. Data are fitted with the scattering intensities expected from objects of simple geometry (a) and according to the fuzzy sphere model, which has been used to describe the dendrimers (b).

are given in Figure 8-6a showing that none of the three geometries give accurate results for the whole observable $q$ range. In Figure 8-6b, experimental data are also fitted to the fuzzy sphere model discussed in chapter 6.2. Best results are obtained assuming a particle radius of $R \approx 3.5 \mathrm{~nm}$ and a surface fuzziness of $\sigma \approx 1.6 \mathrm{~nm}$. Although this model is successfully characterizing dendrimers in solution, it does not allow for a reasonable description of the PAMAM 6/DNA scattering entities. Therefore, all assumed models are incapable to describe the experimental data properly over the whole observable $q$ range. However, from the fact that the PAMAM 6/DNA X-ray pattern can be well approximated for initial scattering vector values $q<0.8 \mathrm{~nm}^{-1}$, it can be concluded that above fits at least offer a rough estimate of the overall particle dimensions. Independent of the assumed geometry, particle dimensions are clearly larger than those of a single PAMAM 6 dendrimer $\left(R_{\mathrm{P} 6}=3.04 \mathrm{~nm}\right)$.

\subsubsection{Determination of the pair distance distribution function}

In order to obtain a real-space representation of the scattering data, the pair distance distribution function $p(r)$ is calculated. $p(r)$ gives the probability of finding a pair of small elements at a distance $r$ within the entire volume of the scattering entity. Hence, the approximated pair distance distribution function $p(r)$ provides information about the conformation of scattering entities. A Fourier transformation connects the measured scattering intensity $I(q)$ to $p(r):^{.70,228}$ 


$$
I(q)=4 \pi \int_{0}^{\infty} p(r) \frac{\sin (q r)}{q r} d r
$$

In principle, $p(r)$ and $I(q)$ contain the same information. However, a real-space representation is often more intuitive and information about the particle shape and conformation are more easily deduced by straightforward visual inspection of $p(r) .^{70}$

Direct Fourier transformation requires scattering data from $q=0$ to infinity. Experimental data $I(q)$, however, contain only a finite number of points in an interval $\left[q_{\min }, q_{\max }\right]$. The precision of these measurement points is determined by the corresponding statistical errors as well as by systematic errors, in particular due to instrumental effects (finite beam size, divergence,...). In addition, an appropriate background correction is of significant importance. ${ }^{229}$

Due to the experimental precision and the finite experimental range of scattering vectors, it is not possible to derive $p(r)$ by a direct inverse Fourier transformation. Therefore, small angle scattering data treatments have been developed for the purpose of extracting the maximum available information about analyzed systems. An indirect Fourier transformation (IFT) method of reciprocal space scattering data into real space is able to minimize the effects of missing data. ${ }^{70,230}$ The result of these calculations are a smooth fit to the smeared data, a desmeared scattering function, and the pair distance distribution function $p(r)$. Since $p(r)$ directly reflects errors due to concentration effects (negative oscillations around the maximum distance) and insufficiently removed scattering background (small increase near $r=0$ ), IFT offers the possibility to minimize these influences on the course of $p(r) .^{231}$

Here, the program GNOM is used to evaluate $p(r)$ from recorded SAXS curves. ${ }^{76,232}$ After subtraction of the background and the averaged solvent (water), GNOM reads in 1D scattering curves possibly smeared due to beam divergence and instrumental distortions. The smeared data can be corrected for instrumental broadening by numerical desmearing with the measured beam cross-section profile. The theoretical aspects of this method - i.e. the main equations relating the scattering intensity to the distribution functions and the description of smearing effects - and its experimental applications are discussed elsewhere. ${ }^{70,76,229,232,233}$

From the sampling theorem, predictions can be made about the minimal and maximal length scale, $r_{\min }$ and $r_{\max }$, which can be resolved in real space by IFT of a certain data set with scattering vector values in the interval $\left[q_{\min }, q_{\max }\right]$ and a step width of $\Delta q$ :

$$
r_{\max } \leq \frac{\pi}{\Delta q} \text { or } r_{\max } \leq \frac{\pi}{q_{\min }}
$$



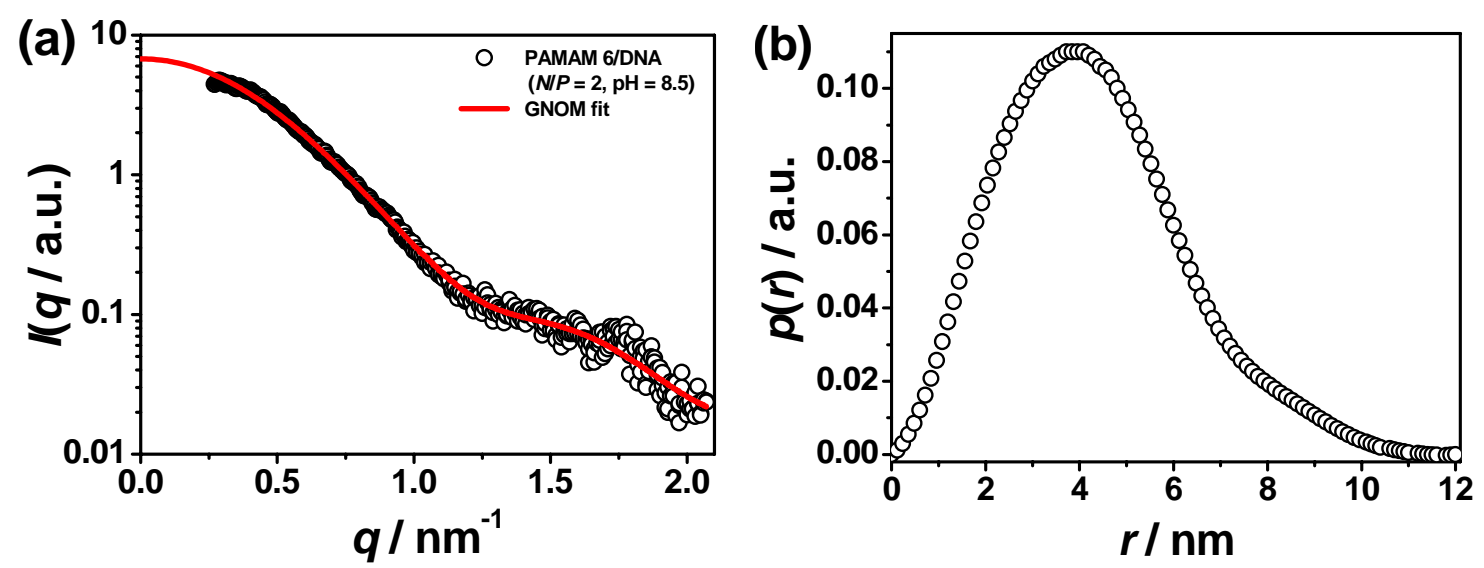

Figure 8-7: (a) Experimental data recorded from PAMAM 6/DNA fitted using GNOM. (b) Corresponding pair distance distribution function $p(r)$ of PAMAM 6/DNA calculated with GNOM.

$$
r_{\min }=\frac{\pi}{N \Delta q} \text { or } r_{\min }=\frac{\pi}{q_{\max }}
$$

However, for most cases, it has been shown that

$$
r_{\min } \approx \frac{1}{q_{\max }}
$$

provides a better estimation of $r_{\min }{ }^{233}$ Here, $q_{\min }=0.26 \mathrm{~nm}^{-1}$ and $q_{\max }=2.08 \mathrm{~nm}^{-1}$ are the limiting factors. Corresponding upper and lower resolution limits are $r_{\max } \approx 15.0 \mathrm{~nm}$ and $r_{\text {min }} \approx 0.5 \mathrm{~nm}$, respectively.

In Figure 8-7a, the GNOM fit to the experimental data is given showing good agreement. Figure 8-7b shows the calculated pair distance distribution function $p(r)$. $p(r)$ displays a bell-shaped course with a stretched tail at higher $r$ values. The maximum is situated at $r=3.9 \mathrm{~nm}$. Bell-shaped $p(r)$ functions are known from globular particles with the position of the maximum corresponding to the particle radius $R$. The stretched tail, which extends over approximately $4 \mathrm{~nm}$, is indicative of an elongated structure with typically skewed distributions for larger $r$.

Since $p(r)$ is zero for distances larger than the particle dimensions, $p(r)$ can account for the maximum extension $D_{\max }$ of the scattering entities. However, for the calculation of $p(r)$, GNOM requires an estimation of $D_{\max }$. Although the choice of maximum particle radius is somewhat subjective, its choice affects the calculated distributions only slightly. It has been shown that an overestimation of the true particle dimension does not lead to significant changes in $p(r)$, whereas underestimation of $D_{\max }$ leads to strong 
oscillations of $p(r) .{ }^{231}$ An approximate estimate of $D_{\max }$ is usually known a priori and can be iteratively refined.

The maximal particle extension is determined to $D_{\max } \approx 12 \mathrm{~nm}$. The value clearly lies below the theoretical upper resolution limit $r_{\max }$ of the IFT method. In these experiments, relatively long DNA chains have been used (chapter 5). Therefore, it is important to note that the $D_{\max }$ value extracted from $p(r)$ corresponds to the maximal overall diameter of the "compact part" of the scattering entities, i.e. the entity formed by the dendrimer and the stretch of DNA with that it interacts (chapter 8.2.3). Due to the relatively low fraction of scattering mass present in the DNA tails compared with the central part, the tails contribute only marginally to the scattering intensity and are not accounted for by $D_{\max }$. Similar observations are reported in literature for NCPs. ${ }^{234}$ With a height of $H_{\mathrm{NCP}}=11 \mathrm{~nm}$ and a radius of $R_{\mathrm{NCP}}=3.75 \mathrm{~nm}$, NCPs and PAMAM 6/DNA scattering entities possess similar dimensions. Moreover, histone octamers (net positive charge of $142 \mathrm{e}^{+}$) and PAMAM 6 at $\mathrm{pH}=8.5$ carry a comparable amount of positive charges. Therefore, a comparison of both seems reasonable. In order to compare the PAMAM 6/DNA scattering entities to NCPs, the program CRYSOL ${ }^{235}$ is used to calculate the scattering intensity $I(q)$ of an ideal solution of NCPs from the crystallographic coordinates published by Harp et al. (protein data base file leqz.pdb). ${ }^{236}$

In Figure 8-8a, the corresponding $I(q)$ curve is scaled to the same intensity at the origin, $I(0)$, as $I(q)$ of PAMAM 6/DNA in Figure 8-7. Figure 8-8b shows the corresponding pair distance distribution function $p(r)$. Exhibiting a bell-shaped distribution with a stretched tail at high $r$ values, $p(r)$ obtained from PAMAM 6/DNA entities and NCPs share common features. However, there are obvious differences, e.g. for NCPs the maximum of $p(r)$ is situated at $r \approx 4.3 \mathrm{~nm}$. Analogue to PAMAM 6/DNA scattering entities, a maximal particle extension of $D_{\max } \approx 12 \mathrm{~nm}$ is observed.

As $p(r)$ corresponds to the distribution of distances $r$ between any two scattering elements within a particle, it also offers an alternative way of calculating the radius of gyration $R_{\mathrm{g}}{ }^{70}$

$$
R_{g}^{2}=\frac{\int_{0}^{D_{\max }} p(r) r^{2} d r}{2 \int_{0}^{D_{\max }} p(r) d r}
$$

Compared to the Guinier approximation, the use of IFT yields more reliable results since it is less sensitive to the data cut-off at small angles. ${ }^{229}$ Obtained values for the radii of gyration are $R_{\mathrm{g}}(\mathrm{PAMAM} 6 / \mathrm{DNA})=3.3 \mathrm{~nm}$ and $R_{\mathrm{g}}(\mathrm{NCP})=3.7 \mathrm{~nm}$. 

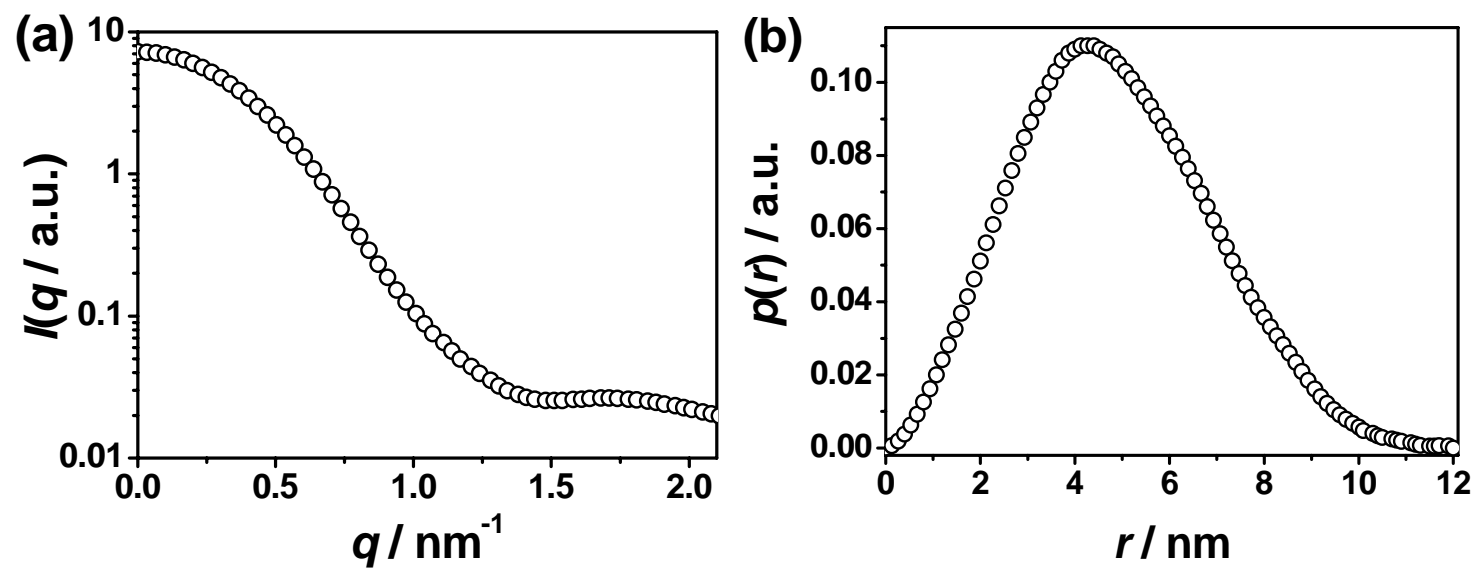

Figure 8-8: (a) Scattering intensity of a dilute NCP solution calculated from the crystallographic data (protein data base file 1eqz.pdb) using CRYSOL. (b) Corresponding pair distance distribution function $p(r)$ of NCPs calculated with GNOM.

With $R_{\mathrm{g}}$ calculated from the IFT data for PAMAM 6/DNA at hands, it is possible to compare the result from this model-independent approach to those obtained from fitting the experimental data with the scattering intensity expected from particles of simple geometry $\left(R_{\mathrm{g}}=3.0-3.1 \mathrm{~nm}\right.$, Figure 8-6a). Although PAMAM 6/DNA entities are of similar dimensions as these particles, the $R_{\mathrm{g}}$ value obtained from the $p(r)$ analysis is about $10 \%$ larger, independent of the assumed geometry (spherical, cylindrical, and ellipsoidal) of the scattering entities. This indicates that - contrary to the assumed simple geometries - PAMAM 6/DNA scattering entities contain protuberances, which must contribute to an increase of $R_{\mathrm{g}}$ compared to the values obtained from the smooth model objects. ${ }^{237}$

\subsubsection{Ab initio modeling of PAMAM 6/DNA entities at $\mathrm{pH}=8.5$}

Recently, small angle scattering data based modeling approaches allow for computation of low-resolution structural models of randomly oriented biological macromolecules in solution. ${ }^{75}$ Since up to now high-resolution crystallographic data are not available for most biological systems, visualization with such low resolution models is of significant importance. Here, the program DAMMIN ${ }^{75,80}$ is used to establish low-resolution bead models of the 3D shapes of PAMAM 6/DNA scattering entities in solution. Using compactness and connectivity constraints, models are exploited ab initio from the experimental SAXS data without needing any a priori structural information. With the 


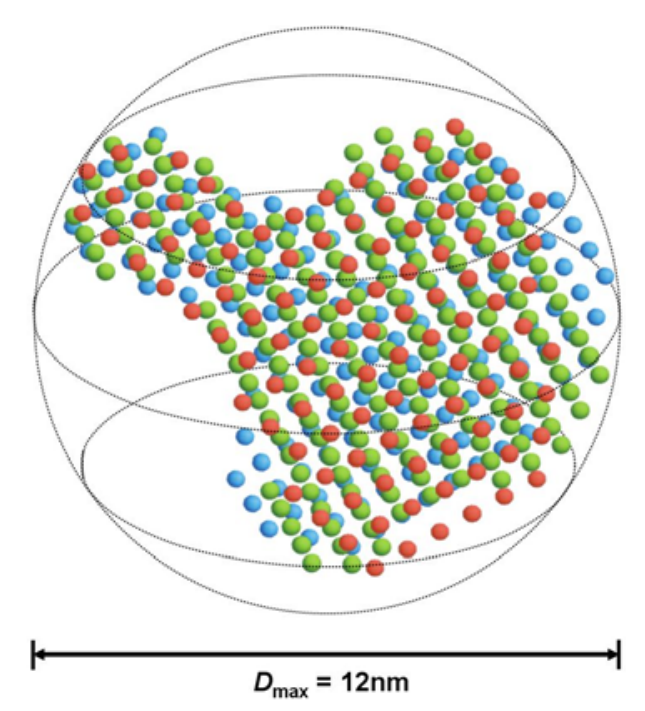

Figure 8-9: Superposition of three final configurations obtained from independent runs. The dashed sphere gives the search volume.

GNOM output file as input, DAMMIN starts with a spherical search space of $R \approx D_{\max } / 2$ filled with $N \approx 2000$ densely packed beads (dummy atoms) of a radius of $R_{\text {bead }} \approx 0.4 \mathrm{~nm}$, which completely encloses the particle. Simulated annealing is used as a global minimization algorithm that finally achieves a particle configuration matching the SAXS data. Annealing yields stable results for different, random starting points. The shape is recovered in an arbitrary orientation and handedness, the enantiomorph yielding the same scattering curve. ${ }^{75}$

The program RASMOL ${ }^{238}$ is adopted for the graphical representation of the obtained bead models. Figure 8-9 gives a superposition of final conformations of three independent runs. Results yield very similar compact solutions for different starting approximations. Averaging the results is used to further refine the solution. Such a strategy is a legitimate procedure to accumulate overall information and to achieve a sufficient degree of reliability because of the limited resolution of the SAXS experiments and the unsafe of $3 \mathrm{D}$ averaged information from specimens in solution. ${ }^{239}$ The reconstructed shape obtained from averaging of six independent runs is illustrated in Figure 8-10. The bead model restored by the shape determination program DAMMIN matches the observed $I(q)$ data in the angular range $q<1.4 \mathrm{~nm}^{-1}$, whereas the program is not able to fit the high $q$ portion of the experimental scattering pattern accurately (Figure 8-10a). However, it still provides a fair approximation of the overall appearance of the scattering entities. ${ }^{80,229}$ 

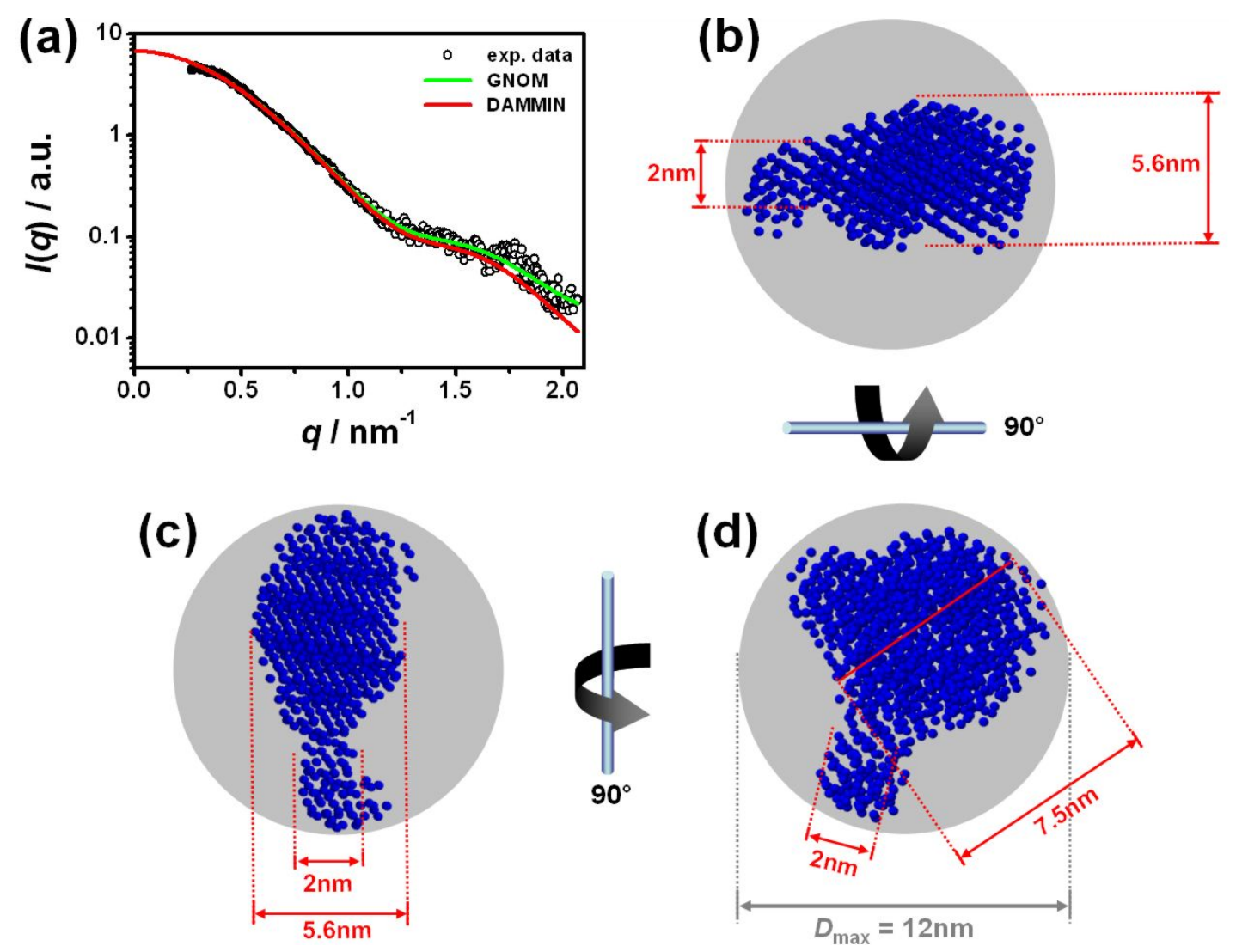

Figure 8-10: (a) PAMAM 6/DNA X-ray profile fitted with GNOM and DAMMIN, respectively. (b)-(d) ab initio bead model of the scattering entities performed using DAMMIN. Grey circles give the spherical search volume $\left(R \approx D_{\max } / 2\right)$

The bead model yields positive proof of a DNA wrapping scenario. Compared to dendrimers employed in chapter 7, the tendency to maximize the charge matching drives the DNA chains to (partially) wrap around the significantly larger and higher charged PAMAM 6. In Figure 8-10, the most obvious structural feature of PAMAM 6/DNA scattering entities is an ellipsoidal structure with a cross-section of approximately $a_{\text {DAMMIN }}=7.5 \mathrm{~nm}$ and a height of about $b_{\text {DAMMIN }}=5.6 \mathrm{~nm}$. Due to its dimensions and shape, the ellipsoidal structure is assigned to comprise the dendrimer $\left(2 \cdot R_{\mathrm{P} 6} \approx b_{\mathrm{DAMMIN}}\right)$ and the tightly bound stretch of DNA $\left(d_{\mathrm{DNA}}+2 \cdot R_{\mathrm{P} 6} \approx a_{\mathrm{DAMMIN}}\right)$. Assigning a $d_{\mathrm{DNA}}=2.0 \mathrm{~nm}$ hard-core diameter to the DNA, ${ }^{53}$ these results suggest a PAMAM 6 dendrimer radius of

$$
R_{P 6} \approx \frac{a_{D A M M I N}-d_{D N A}}{2} \approx \frac{b_{D A M M I N}}{2}=2.8 \mathrm{~nm}
$$

At $\mathrm{pH}=8.5$ - the solution $\mathrm{pH}$ used for PAMAM 6/DNA measurements - however, a larger radius of $R_{\mathrm{P} 6}(\mathrm{pH}=8.5)=3.04 \mathrm{~nm}$ has been observed. This indicates that 
PAMAM 6 dendrimers shrink upon the interaction with the oppositely charged DNA. The value of $R_{\mathrm{P} 6}$ estimated for the dendrimers in PAMAM 6/DNA complexes is close to the radius of an uncharged dendrimer at $\mathrm{pH}=12.5, R_{\mathrm{P} 6}(\mathrm{pH}=12.5)=2.7 \mathrm{~nm}$ (chapter 6.4). The fact that PAMAM 6 dimensions in the complex are corresponding to those of the uncharged dendrimer is in good agreement with results of Brownian dynamics simulations of complexes formed by charged PAMAM 4 dendrimers and oppositely charged linear polymer chains. ${ }^{240}$

The shape and location of the ellipsoidal structure comprising the dendrimer and the tightly bound stretches of DNA is well recovered in all runs (Figure 8-9). However, there is a relatively large uncertainty in the representation of the edges of the ellipsoid, i.e. in the location of the DNA. Such an uncertainty is also observed for the two smaller structures with a diameter of approximately $2 \mathrm{~nm}$. Due to their dimensions, both are assigned as unbound DNA stretches. The occurrence of these uncertainties are not surprising, given the fact that they are only a few percent of the model volume. ${ }^{75}$

According to theory, for spherical objects with dimensions and charges corresponding to PAMAM 6 such a stable dendrimer-DNA complex, in which the polymer wraps around the sphere, is generally expected on single particle level. ${ }^{241}$ In addition, from studying the competition of dendrimers and ethidium bromide binding to DNA, wrapping of DNA around dendrimers has already been speculated to occur for PAMAM dendrimers generation $7 .{ }^{242}$ Moreover, such a wrapping scenario can account for the observed fundamental change in DNA compaction mechanisms with significantly reduced reaction kinetics.

It is tempting to compare the averaged bead model of PAMAM 6/DNA scattering entities to the structure of NCPs obtained from crystallographic X-ray diffraction data (protein data base file 1eqz.pdb). ${ }^{236}$ In order to allow for direct comparison, Figure 8-11 shows the DAMMIN model of PAMAM 6/DNA scattering entities (a), the structure of NCPs obtained from the crystallographic data (b), and a superposition of both (c). In NCPs, a total of 146 base pairs are wrapped around the histone core in approximately 1.7 turns. From Figure 8-11c, it is possible to conclude that a reduced amount of DNA base pairs is wrapped around PAMAM 6 in a way such that the dendrimer is not completely encompassed by the DNA. According to the proposed structure of PAMAM 6/DNA scattering entities, about $12 \mathrm{~nm}$ of DNA $(\approx 35$ base pairs $)$ are adsorbed on each dendrimer. Based on the derived bead model, a schematic representation of the proposed structure of the PAMAM 6/DNA scattering entities is given in Figure 8-11d.

One can envision several different scenarios for the interaction of PAMAM 6 molecules and DNA. Netz and Joanny introduced different states of interaction 
(a)
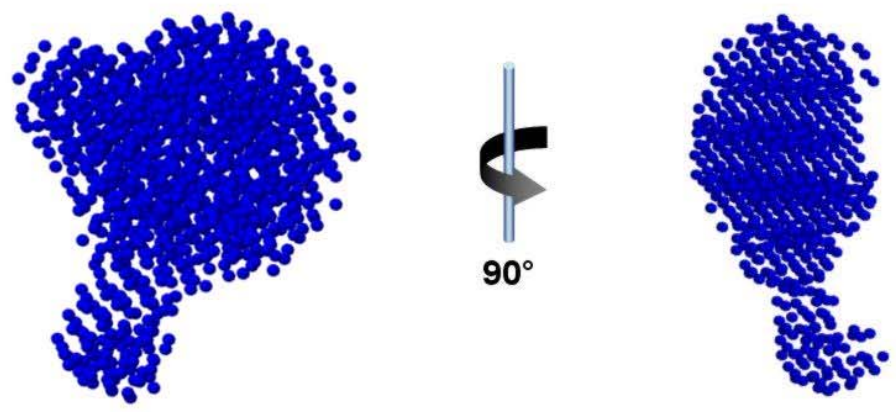

PAMAM 6/DNA

(DAMMIN)

(b)
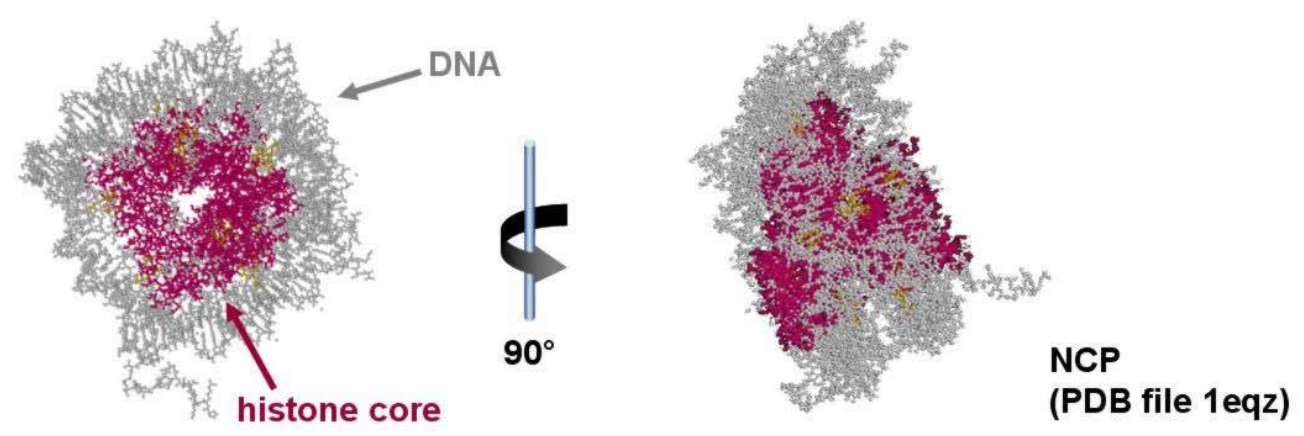

(c)
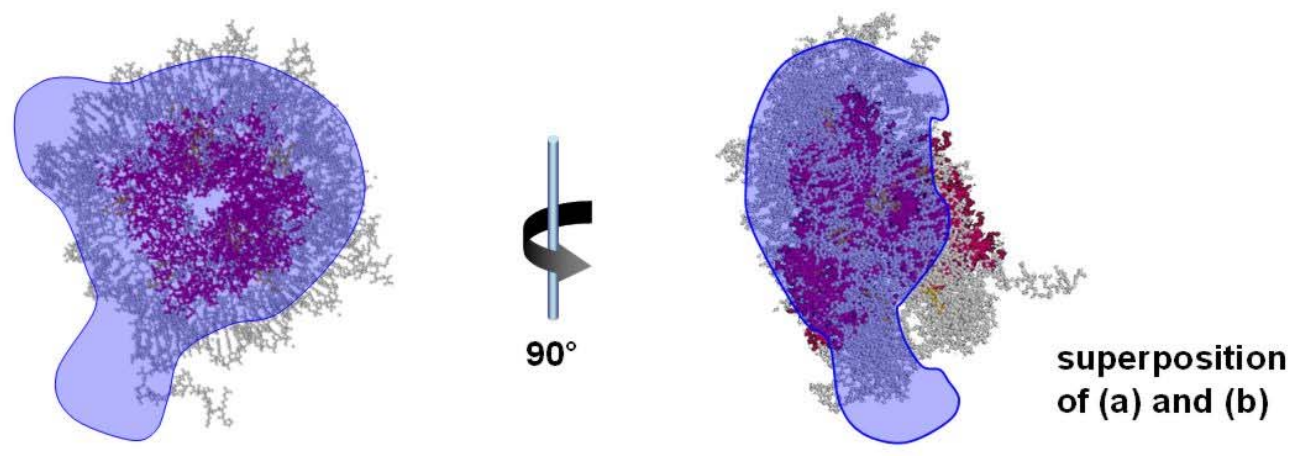

(d)
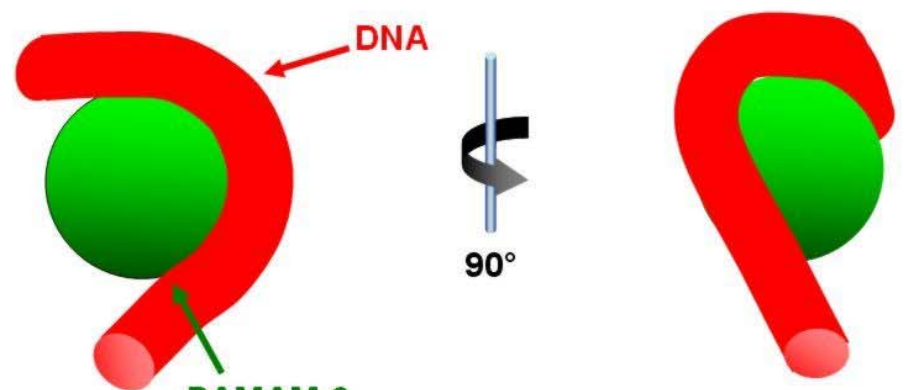

PAMAM 6/DNA

(proposed model)

Figure 8-11: Comparing the bead model of PAMAM 6/DNA and NCPs. (a) PAMAM 6/DNA, (b) NCP, (c) superposition of (a) and (b), (d) Schematic representation of the proposed structure of PAMAM 6/DNA entities. 
between a polyelectrolyte and a charged nanosphere: point touching, adsorption of a finite length of DNA on the nanosphere surface, and full wrapping. ${ }^{243}$ In addition, one dendrimer may simultaneously bind to two or more different DNA chains. Such a binding scenario may compact DNA to a higher degree, and it requires less bending of the DNA double strand. Therefore, a multitude of slightly different conformations of single scattering entities may contribute to the form factor.

Surprising in this context is the fact that the DAMMIN model indicates a rather specific conformation. One may speculate that there are distinct energetic constrains that favor such a rather specific wrapping path of the DNA around the PAMAM 6 dendrimers. This is compatible with the observation of a highly ordered, 3D superstructure of PAMAM 6/DNA complexes at high concentrations (discussed in chapter 8.3). The presence of a significant amount of polydispersity would prevent an arrangement in such a well defined 3D lattice. However, it is not self-evident that the scattering entities and hence the observed form factor remain unchanged in dense complexes at higher concentrations.

\subsection{Exceptional structure of PAMAM 6/DNA complexes}

\subsubsection{D and 3D hexagonal organization of PAMAM 6/DNA complexes}

The structural organization of PAMAM 6/DNA complexes at $\mathrm{pH}=8.5$ is studied using samples with a solute mass fraction of $w / w=2 \%$. Owing to the higher mass fraction used, complex precipitation from solution is observed for all samples. In order to improve the quality of the X-ray patterns, scattering data are recorded from the precipitate phase with a significantly higher solute mass fraction. In addition, the solute mass fraction can be increased by centrifugation.

Figure 8-12 shows the X-ray patterns of PAMAM 6/DNA complexes at $N / P=0.5$ (bottom) and $N / P=1$ (top). As discussed in chapter 8.1.2, the broad correlation peak at $q_{\mathrm{nem}}=1.12 \mathrm{~nm}^{-1}$ corresponds to a nematic liquid-crystalline phase with a spacing of $d_{\text {nem }}=5.6 \mathrm{~nm}$. Increasing the dendrimer content, PAMAM 6/DNA complexes formed in bulk at $N / P=1$ exhibit three small-angle Bragg reflections at $q=1.103,1.907$, and 


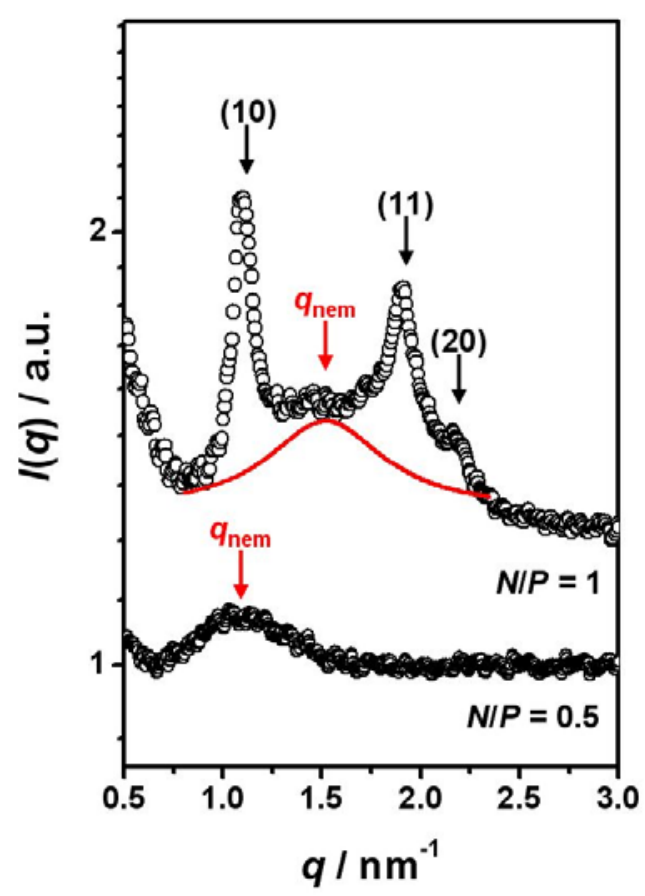

Figure 8-12: Small angle $X$-ray diffraction scans of PAMAM 6/DNA complexes at $N / P=0.5$ and $N / P=1$. All reflections are indexed.

$2.195 \mathrm{~nm}^{-1}$. These $q$-positions are in ratios close to $1: \sqrt{3}: \sqrt{4}$ suggesting a $2 \mathrm{D}$ columnar hexagonal organization of complexes. Consistently, the reflections are indexed as $(h k)=(10),(11)$ and (20). The $2 \mathrm{D}$ lattice constant is $a_{\mathrm{H}}=7.0 \mathrm{~nm}$. Additionally, a broad reflection (dashed line) centered at $q_{\mathrm{nem}}=1.52 \mathrm{~nm}^{-1}$ is observed $\left(d_{\text {nem }}=4.1 \mathrm{~nm}\right)$. This can be interpreted in terms of a coexistence of the $2 \mathrm{D}$ hexagonal phase with a nematic phase. With increasing $N / P$, the broad peak at $q_{\text {nem }}$ disappears and multiple peaks become evident, completing the transition of PAMAM 6/DNA complexes from a (condensed) nematic phase to a long-range ordered liquid-crystalline mesophase. In the 2D columnar hexagonal phase, PAMAM 6/DNA entities are suggested to be piled up regularly into columns that form a hexagonal lattice. However, entities are free to slide along the column exhibiting no intra-columnar correlation. The hexagonal symmetry of PAMAM 6/DNA complexes at $N / P=1$ does not correspond to the square symmetry observed for DNA complexes formed with PPI 4, PPI $5,^{53}$ PAMAM 3 and PAMAM 4, ${ }^{54}$ respectively (chapter 7). This change in symmetry is believed to directly reflect the change in the DNA compaction mechanism associated with increasing dendrimer generation to six.

Figure 8-13a shows the X-ray patterns obtained from PAMAM 6/DNA complexes at $N / P=4.5$. At this complex composition, a maximum amount of eleven sharp 


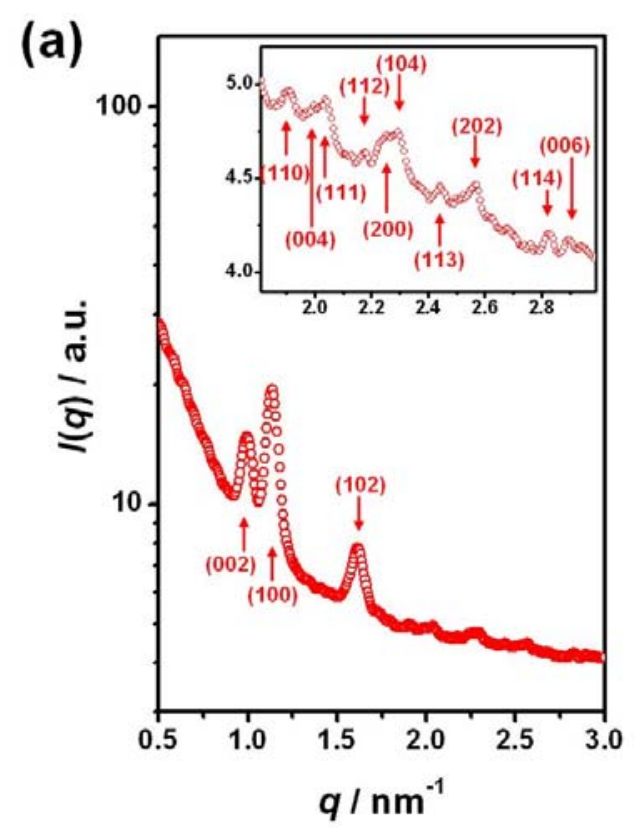

experimental data

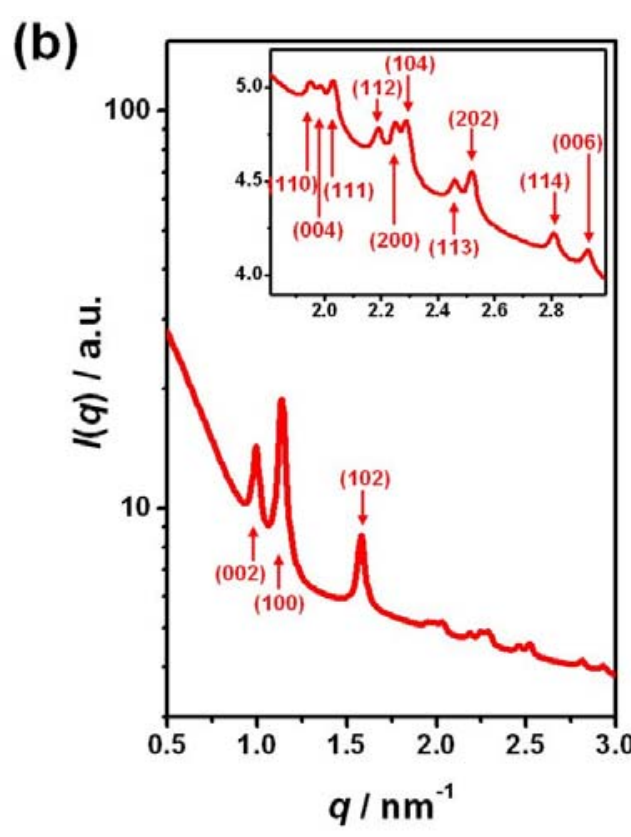

model calculation

Figure 8-13: (a) Small angle X-ray diffraction scans of PAMAM 6/DNA complexes at $N / P=4.5$. All reflections are indexed. (b) Model calculations of the scattering from PAMAM 6/DNA complexes exhibiting a 3D hexagonal symmetry. In the case of degenerated peaks (e.g. (100) and (010)), only a single label is displayed for the sake of clarity.

reflections are observed in the range of $q=0.5-3.0 \mathrm{~nm}^{-1}$. The surprisingly large number of Bragg reflections is indicative of the existence of a crystalline phase with a high degree of positional order. The sufficient number of reflections in the well resolved Xray diffraction pattern makes it possible to unambiguously identify a $3 \mathrm{D}$ hexagonal lattice. The lattice constants are $a_{\mathrm{H}}=6.4 \mathrm{~nm}$ and $c_{\mathrm{H}}=12.6 \mathrm{~nm}$. Bragg reflections are indexed in Figure 8-13a (upper curve and inset).

Figure 8-13b shows a model calculation of the expected scattering from a PAMAM 6/DNA complex of the assumed 3D hexagonal symmetry with the form factor defined in chapter 8.2. The structure factor peaks are taken to be Lorentzians with fixed width of $\Delta q=0.04 \mathrm{~nm}^{-1}$. Separate peak amplitudes are used to mimic obtained experimental scattering profiles. A good agreement with the experimental Xray profile is obtained. It is important to notice that assuming the proposed $3 \mathrm{D}$ hexagonal lattice can account for a consistent indexation of all observed reflections. Moreover, no reflection expected from such a 3D hexagonal lattice is missing in the recorded diffraction pattern. The fact that the width of the diffraction rings is about constant for all Bragg reflections is advising crystalline ordered domains, since for a 

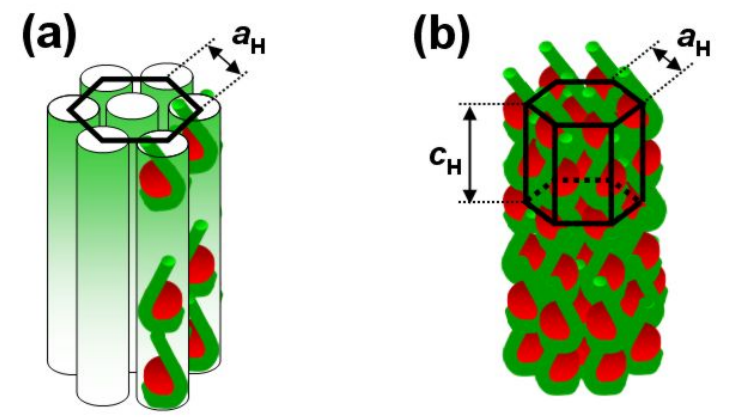

(c)

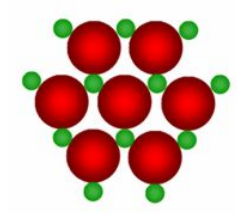

top view

- PAMAM 6 (d)

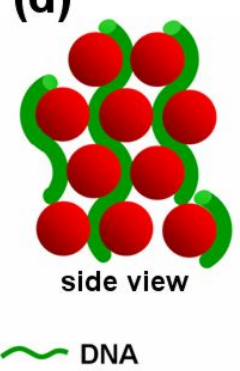

Figure 8-14: Schematic representation of the 2D columnar hexagonal (a) and 3D hexagonal order (b) of PAMAM 6/DNA complexes at $N / P=1$ and 4.5, respectively. (c) and (d) show the top and the side view of the $3 D$ hexagonal order, respectively.

liquid-crystalline-like organization (which would also yield concentric rings) the rings would smear out for higher $q$ values. From the full width at half maximum $\Delta q$ of the (100) reflection, the correlation length $L_{C}=2 \pi / \Delta q$ of the system can be estimated. Taking into account the experimental resolution of $0.03 \mathrm{~nm}^{-1}$, the correlation length is roughly $L_{\mathrm{C}} \approx 650 \mathrm{~nm}$.

A schematic representation of the $2 \mathrm{D}$ columnar hexagonal and $3 \mathrm{D}$ hexagonal arrangement of PAMAM 6/DNA at $N / P=1$ and 4.5, respectively, is given in Figure 8-14. It is important to notice that the used DNA molecules are highly polydisperse in chain length (chapter 5). Thus, the 3D ordering of PAMAM 6/DNA seems to accept such a significant amount of polydispersity in DNA length. However, the formed PAMAM 6/DNA entities seem to be relatively monodisperse as are the dendrimers (chapter 6).

\subsubsection{D to 3D structural transition}

In Figure 8-15, the structure factor of PAMAM 6/DNA complexes of compositions in the range of $N / P=1-4.5$ are shown. It is important to point out that the form factor shown in Figure $8-7 \mathrm{a}$ is exhibiting only minor and unsystematic changes with variations of the complex composition in the studied range of $2 \leq N / P \leq 4.5$. This can be interpreted in a way that the scattering entities composing the complexes are not changing their morphology significantly with $N / P$ but rather rearrange.

Increasing the amount of incorporated dendrimers and hence positive charges, the $\mathrm{X}$ ray patterns given in Figure 8-15 indicate a prominent structural transition of 


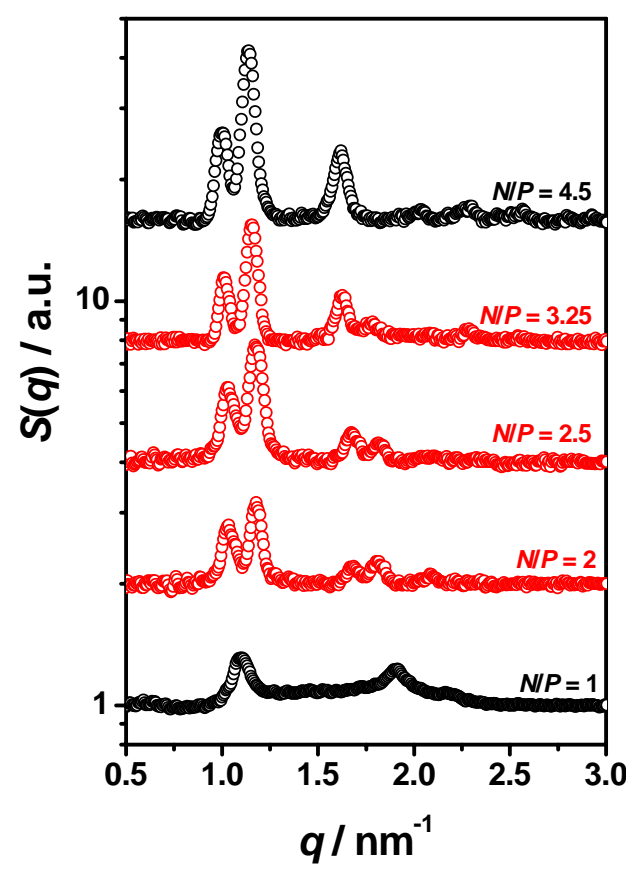

Figure 8-15: Structure factors of PAMAM 6/DNA complexes of compositions in the range of $N / P=1-4.5$.

PAMAM 6/DNA complexes in the range of $1<N / P<2$. The scattering pattern at $N / P=2$ exhibits five sharp Bragg reflections that are incompatible with the $2 \mathrm{D}$ columnar hexagonal order at $N / P=1$. With further increasing the dendrimer content $(2 \leq N / P \leq 4.5)$, PAMAM 6/DNA complexes show gradual changes evolving towards the $3 \mathrm{D}$ hexagonal order observed at $N / P=4.5$. The number of Bragg reflections is continuously increasing up to a maximum of 11 observed at $N / P=3.25$.

Assuming a monophasic structure at $N / P=2$, there are two possible interpretations of the observed changes in lattice symmetry, namely $2 \mathrm{D}$ rectangular or $3 \mathrm{D}$ orthorhombic organization. Rectangular phases sometimes are also called pseudo-hexagonal and crossover from the one symmetry to the other is not unusual. ${ }^{244}$ From the pattern at $N / P=3.25$, the lattice structure can be ruled out to be body-centered orthorhombic. Due to the low information content of small angle X-ray scattering from particles in solutions, the exact space group can - of course - not be determined unambiguously from the data presented in Figure 8-15. However, observed Bragg reflections are best compatible to the space group $I 2{ }_{1} 2_{1} 2_{1}$.

In Figure 8-16, all observed Bragg reflections can be indexed within such lattice symmetry with unit cell parameters $a_{\mathrm{O}}=10.9 \mathrm{~nm}, b_{\mathrm{O}}=6.3 \mathrm{~nm}$, and $c_{\mathrm{O}}=12.4 \mathrm{~nm}$. For $N / P<3.25$, some peaks can only be indexed with difficulty within such lattice symmetry. For all N/P values the low-index (101) peak of the orthorhombic lattice is 


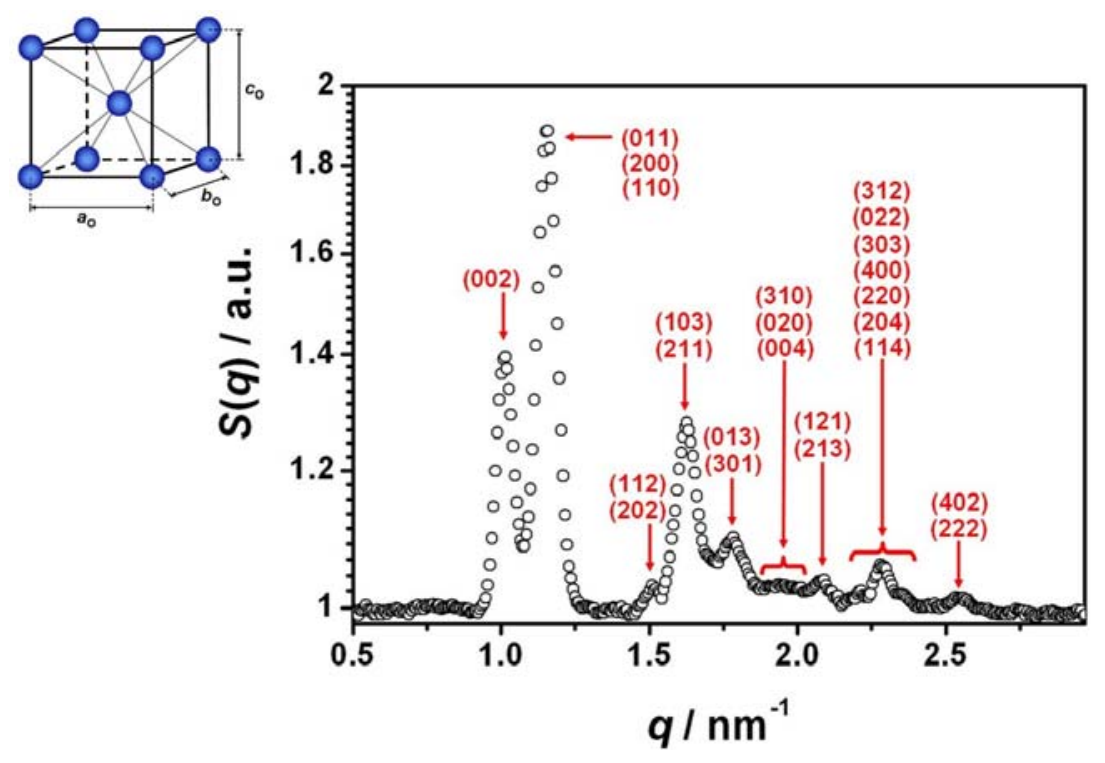

Figure 8-16: Structure factor of PAMAM 6/DNA complexes at $N / P=3.25$. Observed reflections are indexed according to a body-centered orthorhombic unit cell.

missing in the experimental diffraction pattern. However, Figure 8-16 demonstrates that for $N / P \geq 3.25$ this is the only discrepancy when indexing the Bragg reflections. This finding may directly reflect slight distortions of the orthorhombic unit cell. The fact that the consistency of calculated and observed Bragg reflections improves with increasing $N / P$ indicates an improvement of the lattice order of PAMAM 6/DNA complexes.

In Figure $8-17 \mathrm{a}$, values for the lattice parameters $a_{\mathrm{O}}$ and $b_{\mathrm{O}}$ of the rectangular in-plane organization are given in dependence of the $N / P$ ratio. Comparing orthorhombic lattice parameter $a_{\mathrm{O}}$ and hexagonal lattice parameters $a_{\mathrm{H}}$ shows an initial change from $a_{\mathrm{H}}(2 \mathrm{D})=7.0 \mathrm{~nm}$ to $a_{\mathrm{O}}=6.5 \mathrm{~nm}$ at $1<N / P<1.5$. Further increasing $N / P$ yields only minor changes indicating that the organization of PAMAM 6/DNA entities in the $\left(a_{\mathrm{O}}, b_{\mathrm{O}}\right)$-plane stays almost unchanged. Moreover, this suggests that the transition between the orthorhombic order at $N / P=4$ to the hexagonal order at $N / P=4.5$ is a gradual one. Consistently, the ratio $a_{\mathrm{O}} / b_{\mathrm{O}}=1.64-1.71$ is very close to $\sqrt{3}$. This indicates that the $3 \mathrm{D}$ hexagonal organization of PAMAM 6/DNA entities is only slightly distorted.

The organization of PAMAM 6/DNA entities normal to the plane defined by $a_{\mathrm{O}}$ and $b_{\mathrm{O}}$ is determined by the periodicity $c_{\mathrm{O}}$. Values of $c_{\mathrm{O}}$ can be directly derived from the position $q_{\mathrm{c}}$ of the first peak in the diffraction pattern: $c_{\mathrm{O}}=22 \pi / q_{\mathrm{c}}$. The additional factor 2 is due to the reflection conditions of a body-centered orthorhombic unit cell, which 

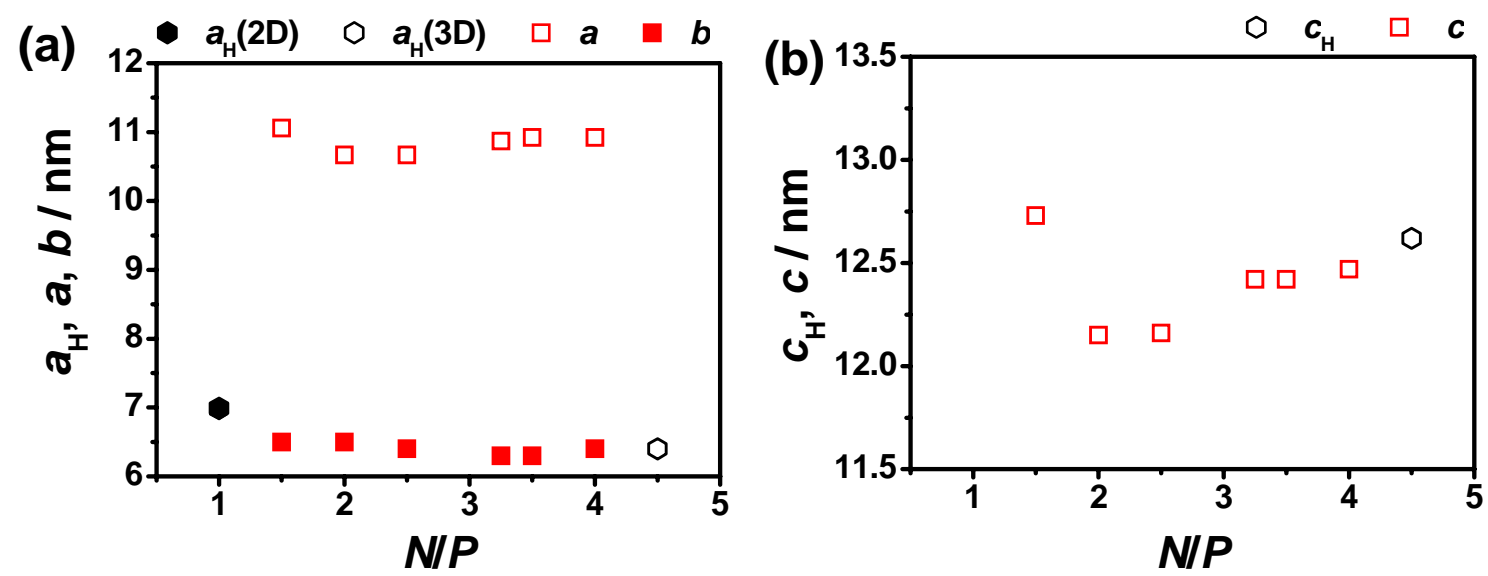

Figure 8-17: N/P dependence of orthorhombic lattice parameters $a, b$, and $c$. 2D and 3D hexagonal lattice parameters $a_{\mathrm{H}}$ and $c_{\mathrm{H}}$ are given for comparison.
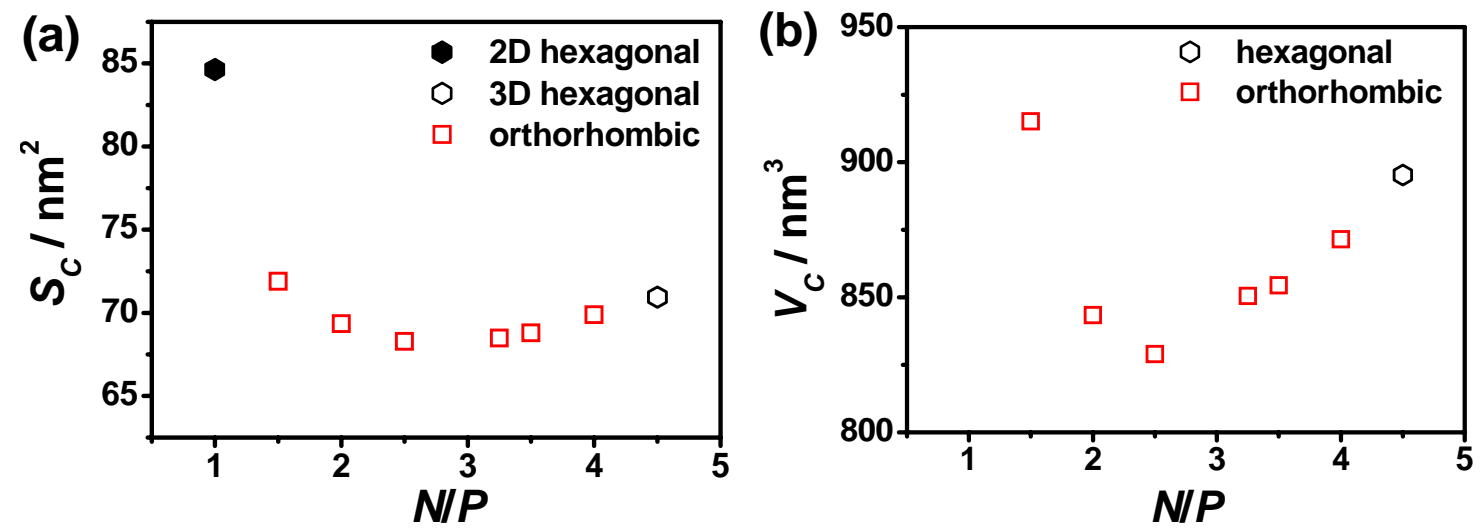

Figure 8-18: $N / P$ dependence of the surface area per column $S_{\mathrm{C}}($ a) and the volume of the unit cell (b).

only allows even values $l=2 n(n \in \aleph)$. In Figure 8-17b, possible values for the parameter $c_{\mathrm{O}}$ are given for the different complex compositions. At $N / P=1.5$, the onset of intra-columnar correlations between neighboring PAMAM 6/DNA entities is reflected by the observed periodicity of $c_{\mathrm{O}}=12.7 \mathrm{~nm}$. With increasing $N / P$, the longitudinal order along the columns is further evolving and a $3 \mathrm{D}$ quasi-hexagonal (orthorhombic) phase is fully established at $N / P=2$. This is consistent with the observed changes of PAMAM 6/DNA organization in the $\left(a_{\mathrm{O}}, b_{\mathrm{O}}\right)$-plane. After reaching its minimum value of $c_{\mathrm{O}}=12.1 \mathrm{~nm}$ at $N / P=2.5$, the periodicity perpendicular to the $\left(a_{\mathrm{O}}, b_{\mathrm{O}}\right)$-plane gradually increases until the $3 \mathrm{D}$ hexagonal order with $c_{\mathrm{H}}=12.6 \mathrm{~nm}$ is fully established at $N / P=4.5$. The observed continuous small variations indicate successive shifts in the molecular organization. 


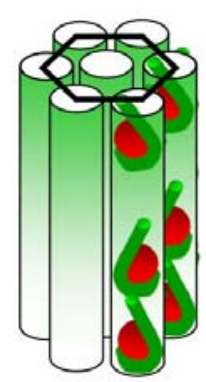

$N I P=1$

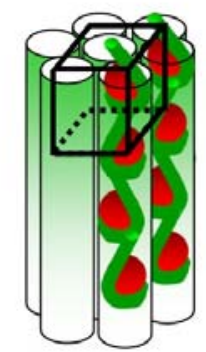

$1<N I P<4.5$

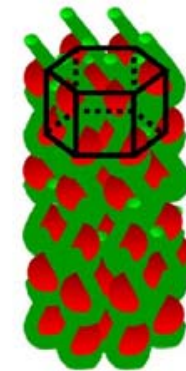

$N I P=4.5$

Figure 8-19: Schematic representation of the structural transition from a 2D columnar hexagonal to a 3D hexagonal organization of PAMAM 6/DNA upon increasing $N / P$ via intermediate orthorhombic states.

From a geometrical point of view, lattice structures are characterized mainly by two parameters: the cross section area $S_{\mathrm{C}}$ of the unit cell in the $\left(a_{\mathrm{O}}, b_{\mathrm{O}}\right)$-plane and the unit cell volume $V_{\mathrm{C}}{ }^{245}$ Both quantities are displayed in dependence of $N / P$ in Figure 8-18. The transition from a $2 \mathrm{D}$ columnar hexagonal to a $3 \mathrm{D}$ orthorhombic order is directly reflected by the significant change of $S_{\mathrm{C}}$ : Increasing $N / P$ from 1 to $1.5, S_{\mathrm{C}}$ is reduced by $15 \%$. $V_{\mathrm{C}}$ follows qualitatively the course of the lattice parameter $c_{\mathrm{O}}$. Interestingly, both $S_{\mathrm{C}}$ and $V_{\mathrm{C}}$ exhibit global minima around $N / P=2.5$.

\subsubsection{Evolution of complex structure with time}

All X-ray patterns presented in Figure 8-13, Figure 8-15, and Figure 8-16 were recorded approximately 13 month after sample preparation. The experimental conditions were designed to produce samples under equilibrium conditions in sealed capillaries. However, a significant evolution of complex structures is observable with equilibration time reflected in dramatic changes of the structure factor $S(q)=I(q) / F(q)$. In order to analyze this phenomenon in more detail, $S(q)$ obtained from complexes with a complex composition of $N / P=4.5$ after different equilibration times are given in Figure 8-20. Immediately after preparation, the sample exhibits a SAXS pattern, which is similar to that observed at $N / P=1$ in Figure $8-13 \mathrm{a}$. The positions of the three reflections around $q=1.05,1.79$, and $2.07 \mathrm{~nm}^{-1}$, respectively, indicate a $2 \mathrm{D}$ columnar hexagonal organization with a lattice constant of $a_{\mathrm{H}}=6.9 \mathrm{~nm}$. A coexistence of the 2D hexagonal phase with a nematic phase is indicated by the broad peak at $q_{\text {nem }}=1.51 \mathrm{~nm}^{-1}$. However, there is an additional peak at $q_{\mathrm{c}}=1.13 \mathrm{~nm}^{-1}$ with a 


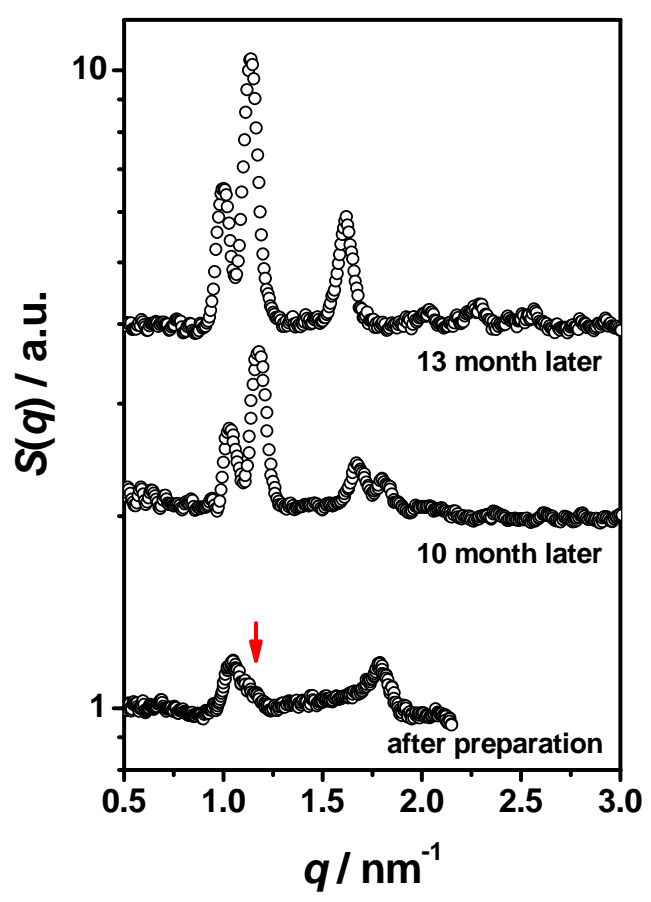

Figure 8-20: Structure factor of PAMAM 6/DNA complexes $(N / P=4.5)$ measured at different times after sample preparation.

significantly higher full width at half maximum of $\Delta q=0.21 \mathrm{~nm}^{-1}$ (indicated by the red arrow in Figure 8-20). The peak at $q=1.05 \mathrm{~nm}^{-1}$ corresponds to the average distance between columns, whereas the peak at $q_{\mathrm{c}}=1.13 \mathrm{~nm}^{-1}$ may correspond to the average spacing between PAMAM 6/DNA entities in a column. Accordingly, this may be indicative for the onset of an organization of PAMAM 6/DNA entities in a 3D ordered phase.

After 10 month of equilibration time, the diffraction pattern is completely different exhibiting six Bragg reflections. The positions of these reflections are incompatible with both a $2 \mathrm{D}$ and a $3 \mathrm{D}$ hexagonal organization. $S(q)$ rather corresponds to diffraction patterns obtained from PAMAM 6/DNA complexes with $2<N / P<3.5$ after 13 month of equilibration (chapter 8.3.2). The transition from $2 \mathrm{D}$ columnar hexagonal to $3 \mathrm{D}$ hexagonal lattice is completed 13 month after sample preparation.

From the data presented in Figure 8-20, it is not possible to be certain that the evolution of PAMAM 6/DNA structure formation is finished after 13 month. It seems more likely that equilibrium values have not been reached yet. Despite possible changes of lattice constants, the highly ordered $3 \mathrm{D}$ hexagonal lattice symmetry is expected to be preserved. The experimental data presented in Figure 8-20 show that the structural organization takes place on surprisingly long time scales of several months. This is consistent with structural organization time scales known from nucleosome core particle (NCP), where equilibration times of more than 17 months have been 
reported. ${ }^{219}$ However, such a long structural organization time makes it impossible to access the complete evolution of PAMAM 6/DNA structure formation in flow.

\subsection{Tuning DNA wrapping}

In chapter 6.4, it has been shown that it is possible to control surface charge density of dendrimers by varying the solvent $\mathrm{pH}$. Such a change is expected to affect the organization of the DNA on the dendrimer surface owing to the electrostatic origin of dendrimer/DNA interactions. Moreover, this should result in changes of both the shape and the size of PAMAM 6/DNA entities. This chapter is dedicated to the analysis of $\mathrm{pH}$ induced effects on the conformation of PAMAM 6/DNA entities and their organization in complexes.

\subsection{1. $\mathrm{pH}$ induced PAMAM 6/DNA complex structure transitions}

Figure 8-21 shows X-ray patterns recorded from PAMAM 6/DNA complexes formed at different $\mathrm{pH}$ conditions. All data are recorded from samples with a fixed DNA to dendrimer concentration ratio of $c_{\mathrm{DNA}} / c_{\mathrm{P} 6}=0.2$. However, owing to the $\mathrm{pH}$ induced variations in dendrimer charge, all samples possess the different $N / P$ ratios given in Figure 8-21. All samples have been equilibrated for 13 month and more. Data are offset for clarity.

The scattering curves presented in Figure 8-21 exhibit significant differences. This indicates that changing $\mathrm{pH}$ results in a dramatic modification of the complex structure. For $\mathrm{pH}=11-14$, all primary and tertiary amino groups of PAMAM 6 dendrimers are uncharged. Therefore, the formation of any DNA-dendrimer complexes is not expected. Consistently, the X-ray pattern recorded at $\mathrm{pH}=14$ does not exhibit any Bragg reflections. Instead, the scattering curve is similar to those obtained from semi-dilute DNA solutions (chapter 5.2).

Reducing $\mathrm{pH}$ results in charging the dendrimers and therefore in an electrostatic attraction of both components. However, the electrostatic attraction is balanced by the elastic energy of the DNA. Corresponding X-ray patterns show that the arising interaction between DNA and dendrimers leads to the formation of PAMAM 6/DNA mesophases. At $\mathrm{pH}=10$, PAMAM 6/DNA mesophases exhibit two reflections at $q=1.03$ and $1.83 \mathrm{~nm}^{-1}$. The ratio $1: \sqrt{3}$ of the two peak positions suggests a $2 \mathrm{D}$ 


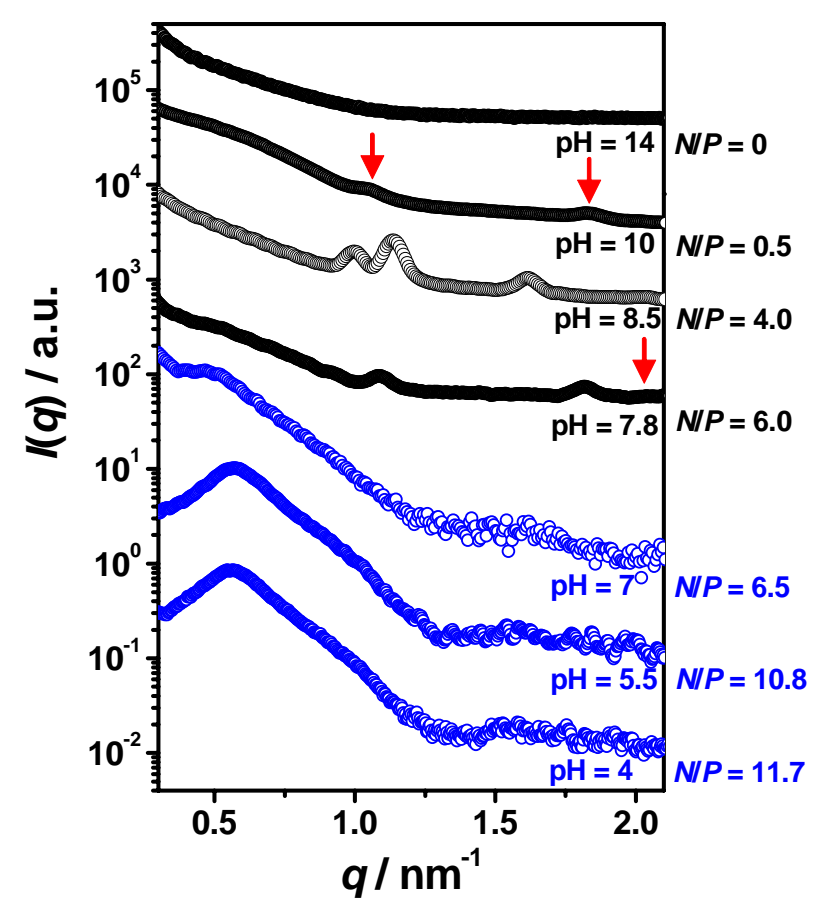

Figure 8-21: Scattering intensity $I(q)$ of PAMAM 6/DNA complexes at different pH values. Arrows indicate weakly pronounced structural features.

columnar hexagonal organization of complexes with an inter-columnar distance of $a_{\mathrm{H}}=7.0 \mathrm{~nm}$. Consistently, the reflections are indexed as $(h k)=(10)$ and (11). The large full width at half maximum $\Delta q_{(10)}=0.25 \mathrm{~nm}^{-1}$ of the (10) reflection indicates that the ordering is restricted to small domains, which likely coexist with unorganized domains. This is supported by the fact that peak intensities are low. A further reduction of the $\mathrm{pH}$ value to $\mathrm{pH}=8.5$ induces a transition from the $2 \mathrm{D}$ columnar hexagonal phase to the $3 \mathrm{D}$ quasi-hexagonal phase discussed in chapter 8.3. Corresponding unit cell parameters are $a_{\mathrm{O}}=10.9 \mathrm{~nm}, b_{\mathrm{O}}=6.3 \mathrm{~nm}$, and $c_{\mathrm{O}}=12.4 \mathrm{~nm}$. Successively, X-ray pattern recorded at $\mathrm{pH}=7.8$, exhibit three reflections at $q=0.98,1.70$, and $1.97 \mathrm{~nm}^{-1}$. These $q$-positions are in ratios close to $1: \sqrt{3}: \sqrt{4}$. Accordingly, the complex structure is transformed back to a $2 \mathrm{D}$ columnar hexagonal ordering, however with a slightly modified lattice spacing of $a_{\mathrm{H}}=7.4 \mathrm{~nm}$.

At neutral $\mathrm{pH}$ conditions, the protonation of all primary amine groups is completed, whereas all tertiary amine groups are unprotonated. Corresponding X-patterns of PAMAM 6/DNA complexes are not exhibiting any Bragg peaks. This indicates that there is no long range order present in the samples any more. However, the scattering intensity $I(q)$ exhibits a downturn for low $q$ values $\left(q<0.6 \mathrm{~nm}^{-1}\right)$. As discussed in chapter 5.2 and 6.2, this reveals the existence of interactions between 


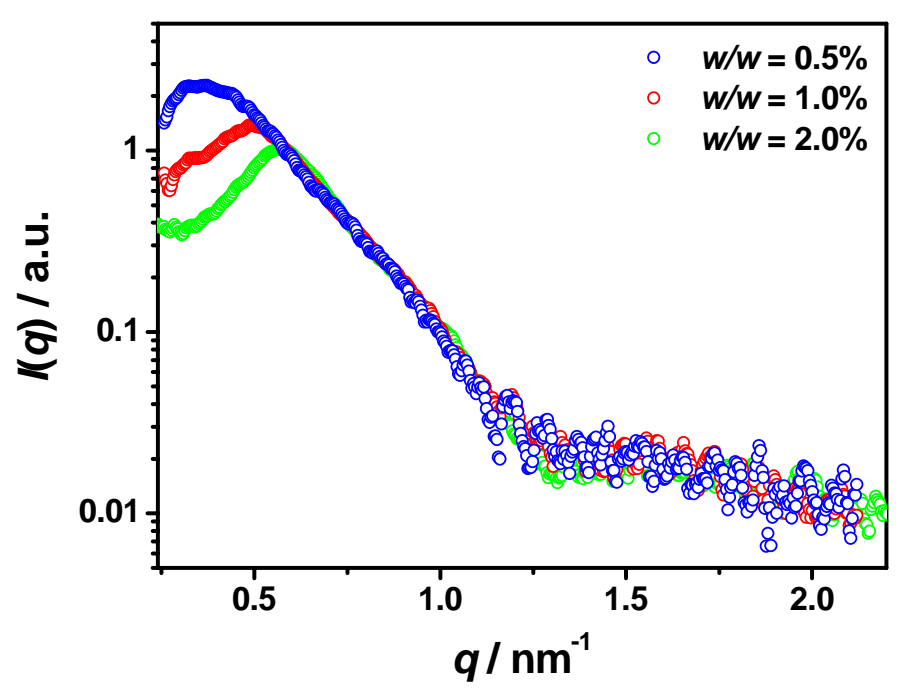

Figure 8-22: X-ray scattering pattern of three aqueous solutions of PAMAM 6/DNA of different mass fraction recorded at $\mathrm{pH}=5.5$. A fixed complex composition of $N / P \approx 1$ is used for all data sets.

the scattering entities, which modify the corresponding form factor $F(q)$ in the low $q$ region. ${ }^{159,234}$ For higher $q$ values, $I(q)$ almost corresponds to $F(q)$.

Inter-particle interactions become stronger at lower $\mathrm{pH}$ conditions, where the tertiary amino groups are continuously protonated. This is reflected by the more pronounced downturn in the low $q$ region. In addition to the loss of long range correlational order, starting at $\mathrm{pH} \approx 7$, the course of $I(q)$ is significantly modified. In particular, the slope for $0.6 \mathrm{~nm}^{-1}<q<1.3 \mathrm{~nm}^{-1}$ is significantly decreased and a minimum around $q \approx 1.3 \mathrm{~nm}^{-1}$ is observed. These features indicate that PAMAM 6/DNA entities increase in size and gain a more compact conformation with increasing dendrimer charge. This is analysed in detail in the following chapter.

\subsubsection{Form factor of PAMAM 6/DNA entities at low pH}

In Figure 8-22, X-ray patterns of aqueous PAMAM 6/DNA solutions obtained at low $\mathrm{pH}$ conditions $(\mathrm{pH}=5.5)$ and a fixed complex composition of $N / P \approx 1$ are shown. As discussed in chapter 2.2, X-ray scattering from dilute solutions of organic macromolecules is usually of very low intensity, in particular for larger scattering angles and hence higher $q$ values. The quality of the X-ray patterns can be improved by 


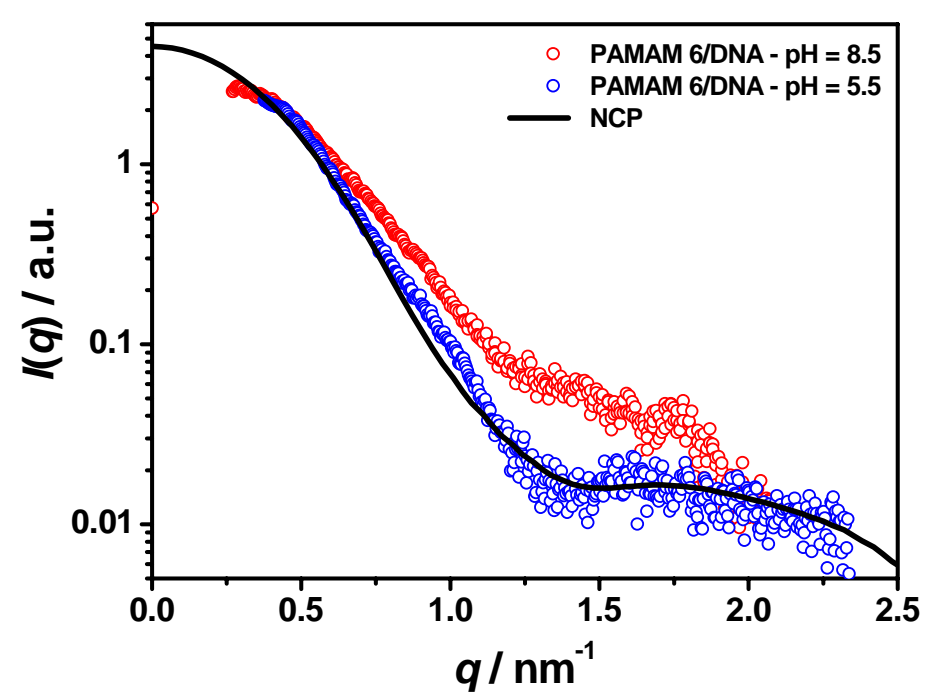

Figure 8-23: Form factor of PAMAM 6/DNA scattering entities at $\mathrm{pH}=5.5$ and $\mathrm{pH}=8.5$. Data are contrasted to the scattering curve obtained from NCPs.

increasing the solute mass fraction $w / w$. However, increasing $w / w$ also modifies the course of the X-ray patterns, in particular in the low $q$ region.

According to observation of PAMAM 6/DNA complexes at $\mathrm{pH}=8.5$, a precipitation from solution can occur. Recording scattering data from the supernatant is again used to diminish inter-particle effects. In Figure 8-22, for all measured mass fractions $(w / w \geq 0.5 \%)$, the scattering intensity $I(q)$ exhibits no Bragg reflections but a significant downturn for low $q$ values. This reveals the existence of net repulsive interactions between PAMAM 6/DNA entities. With increasing PAMAM 6/DNA mass fraction, this downturn is more pronounced directly reflecting the effect of stronger inter-particle interactions on the scattering intensity.

Figure 8-22 shows that although inter-particle effects on the scattering intensity are still present at the lowest mass fraction of $w / w=0.5 \%$, they are already significantly reduced and shifted to lower $q$ values. Corresponding scattering data are used as an approximation of the (ideal) form factor. Consequently, to prevent any effects of the downturn at small $q$ values, the calculation of the pair distance distribution function $p(r)$ and the determination of the radius of gyration $R_{\mathrm{g}}$ are performed using the range $q>0.32 \mathrm{~nm}^{-1}$. In order to improve statistics in the high $q$ region, where inter-particle effects can reasonably be neglected even at higher sample concentrations, the form factor data obtained at $w / w=0.5 \%$ are spliced with corresponding data of higher mass fraction $(w / w=2.0 \%)$. The resulting X-ray pattern is given in Figure 8-23.

In Figure 8-23, the X-ray pattern recorded at $\mathrm{pH}=5.5$ is contrasted to the curve recorded at $\mathrm{pH}=8.5$. Scattering intensities of PAMAM 6/DNA recorded at different 
$\mathrm{pH}$ exhibit very distinct courses and do not superimpose. In particular, the increased slope at low $q$ values and the minimum around $q \approx 1.3 \mathrm{~nm}^{-1}$, which is observed only for the data recorded at low $\mathrm{pH}$ conditions, suggest a noticeable conformational modification of PAMAM 6/DNA scattering entities.

In addition to the X-ray patterns obtained from PAMAM 6/DNA, in Figure 8-23 the scattering curve of NCPs is given as a solid line for comparison. In order to enable better comparability, all data sets are normalized to their values at $q=0.32 \mathrm{~nm}^{-1}$. Contrary to the observed differences between PAMAM 6/DNA scattering entities at different $\mathrm{pH}$ conditions, the experimental scattering intensity of PAMAM 6/DNA at low $\mathrm{pH}$ and the calculated intensity of NCPs extracted from the crystallography data exhibit remarkably similar scattering curves. The most prominent common feature is the superposition of the scattering curves for $q<0.8 \mathrm{~nm}^{-1}$. In addition, the scattering curve of PAMAM 6/DNA shows a minimum at $q \approx 1.3 \mathrm{~nm}^{-1}$, whereas for NCP the minimum position is shifted to only slightly higher $q$ values of $q=1.4 \mathrm{~nm}^{-1}$. Both features indicate that PAMAM 6/DNA at low $\mathrm{pH}$ conditions and NCPs possess similar overall shapes and sizes. However, there are - of course - differences in the fine structure of the scattering entities of the two species. This is reflected e.g. by the slight variation in the $q$ position of the minimum and - more pronounced - by the shoulder at $q=1.02 \mathrm{~nm}^{-1}$, which is only present in the scattering curve of PAMAM 6/DNA at low $\mathrm{pH}$ conditions.

For NCPs, it has been shown that a minimum around $q=1.4 \mathrm{~nm}^{-1}$ is closely related to the super-helical path of the DNA wrapping around the histone octamers. ${ }^{246}$ The disappearance of this feature, which has been experimentally observed for NCPs with modified histone tails, is the signature of a change in the super-helical path of the DNA. ${ }^{246}$ The absence of the minimum has been shown to directly reflect a partial detachment of the DNA. ${ }^{246}$ This is in agreement with observations of PAMAM 6/DNA scattering entities at high $\mathrm{pH}$ conditions, which do not exhibit any pronounced minima and where the DAMMIN model showed only partial DNA wrapping (chapter 8.2).

\subsection{3. $p(r)$ and $R_{\mathrm{g}}$ of PAMAM 6/DNA entities at low $\mathrm{pH}$}

The X-ray pattern given in Figure 8-23 is used to calculate the pair distance distribution function $p(r)$ with GNOM. Figure 8-24a shows that the resulting GNOM fit is able to describe the experimental data properly over the whole observable $q$ range. Figure 8 -24b gives $p(r)$. The experimental data allow access to scattering vector values in the interval $q \in[0.32,2.34]$. According to equations (9-3) and (9-5), this corresponds to 

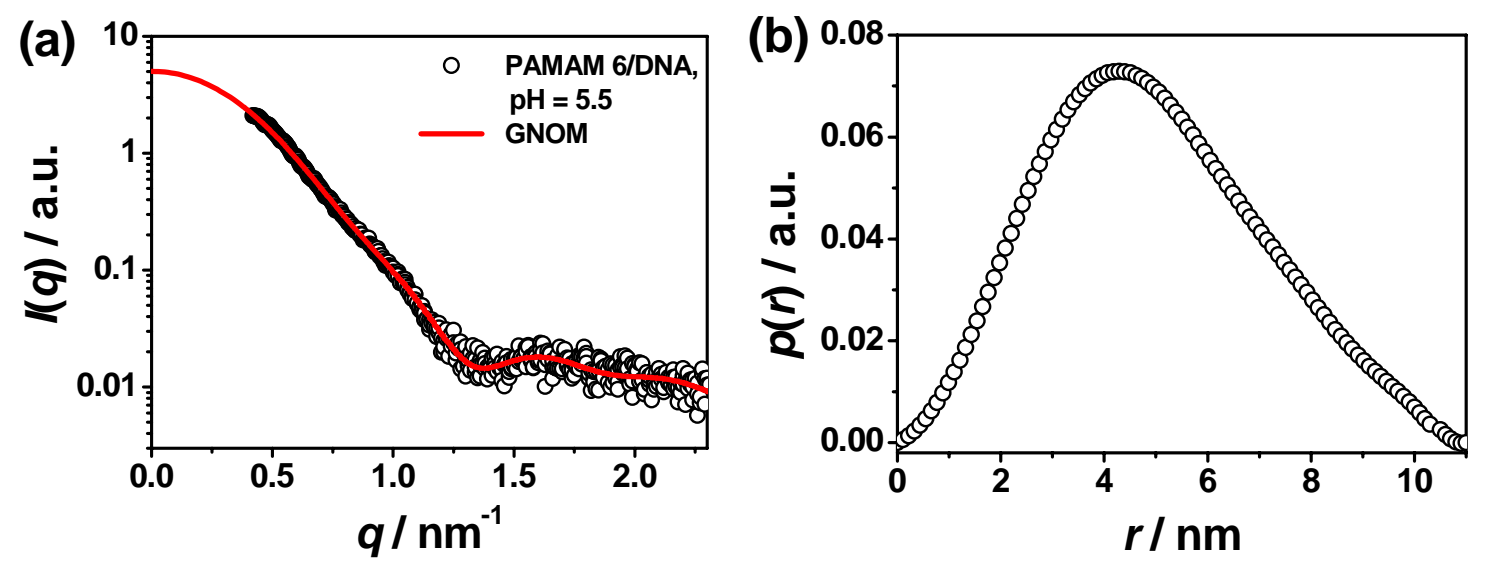

Figure 8-24: (a) GNOM fit to the experimental data obtained from PAMAM 6/DNA at low pH conditions. (b) Calculated pair distance distribution function $p(r)$.

lower and upper resolution limits in real space of $r_{\min } \approx 0.4 \mathrm{~nm}$ and $r_{\max } \approx 10 \mathrm{~nm}$, respectively. The relatively small value of $r_{\max }$ is owing to inter-particle effects, which limit the applicable $q$ range to $q>0.32 \mathrm{~nm}^{-1}$. The maximal particle extension is determined to $D_{\max } \approx 11 \mathrm{~nm}$. This value is slightly higher that the theoretical upper resolution limit $r_{\max }$ of the IFT method.

In order to perform any in-depth analysis of the conformation of PAMAM 6/DNA entities at low $\mathrm{pH}$, the obtained pair distance distribution function is compared to that of PAMAM 6/DNA entities at $\mathrm{pH}=8.5$ and NCPs (Figure 8-25). $p(r)$ of PAMAM 6/DNA entities at low $\mathrm{pH}$ conditions displays an asymmetric bell-shaped course with a maximum at $r=4.3 \mathrm{~nm}$. The slightly skewed distribution for $r>4.3 \mathrm{~nm}$ indicates a rather cylindrical shape. $p(r)$ of PAMAM 6/DNA at low and high $\mathrm{pH}$ differ significantly. The fact that the maximum position of the bell-shaped course is shifted to slightly higher $r$ values indicates larger scattering entities at low $\mathrm{pH}$ conditions. In addition, the significantly less pronounced tail at high $r$ values suggests a more compact overall shape. This is indicative for an altered DNA wrapping scenario: owing to the increased surface charge density of PAMAM 6 at $\mathrm{pH}=5.5$, a larger stretch of DNA is adsorbed on the dendrimer surface. With $D_{\max } \approx 11 \mathrm{~nm}$, maximal extensions of PAMAM 6/DNA entities at $\mathrm{pH}=5.5$ are in reasonable agreement with $D_{\max } \approx 12 \mathrm{~nm}$ observed for both PAMAM 6/DNA entities at $\mathrm{pH}=8.5$ and NCPs.

Consistently to $I(q)$, the course of $p(r)$ of PAMAM 6/DNA entities at low pH exhibits a remarkable similarity to that of NCPs with only slight deviations for $r>7.5 \mathrm{~nm}$. The radius of gyration $R_{\mathrm{g}}$ has been calculated from the $p(r)$ functions. Lowering $\mathrm{pH}$, the value of $R_{\mathrm{g}}$ is increasing to $R_{\mathrm{g}}=3.8 \mathrm{~nm}$. This value is very close to the experimental 


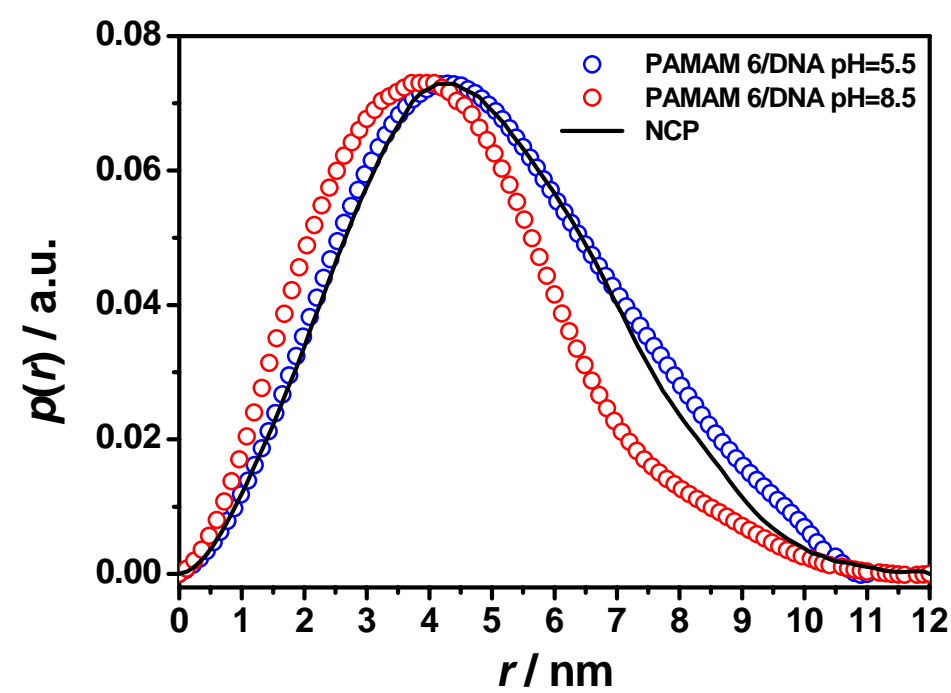

Figure 8-25: Pair distance distribution function $p(r)$ of PAMAM 6/DNA at $p H=5.5$ contrasted to $p(r)$ obtained from PAMAM 6/DNA at $\mathrm{pH}=8.5$ and NCPs.

value obtained for NCPs $\left(R_{\mathrm{g}}=3.7 \mathrm{~nm}\right)$. The fact that the pair distribution functions and the radii of gyration of both samples are nearly identical supports the conclusion that both entities are very much alike.

\subsubsection{Ab initio modeling of PAMAM 6/DNA entities at low $\mathrm{pH}$}

In order to resolve the 3D shape of PAMAM 6/DNA entities at low $\mathrm{pH}$ conditions and to estimate the amount of DNA wrapped around the dendrimers, the program DAMMIN $^{75,80}$ is used to establish low-resolution bead models. In order to improve the reliability of the model and to further refine the solution, averaging of results obtained from independent runs with random initial conditions is used. The shape of the structure is well recovered in all runs.

A graphical representation of the reconstructed shape is given in Figure 8-26. Figure 8-26a shows that the bead model matches the observed $I(q)$ data in the whole experimental $q$ range with good accuracy. Therefore, the model provides a fair reproduction of the shape of the scattering entities. Consistent with suggestions based on the course of $p(r)$, the bead model shows a cylindrically shaped particle with almost circular cross-section. The particle diameter is estimated to $a_{\text {DAMMIN }}=10.8 \mathrm{~nm}$, whereas its height is about $b_{\text {DAMMIN }}=6.6 \mathrm{~nm}$. The resolution of PAMAM 6/DNA entities is sufficient to unambiguously determine a $\mathrm{pH}$ induced transition of the structure of PAMAM 6/DNA entities. 

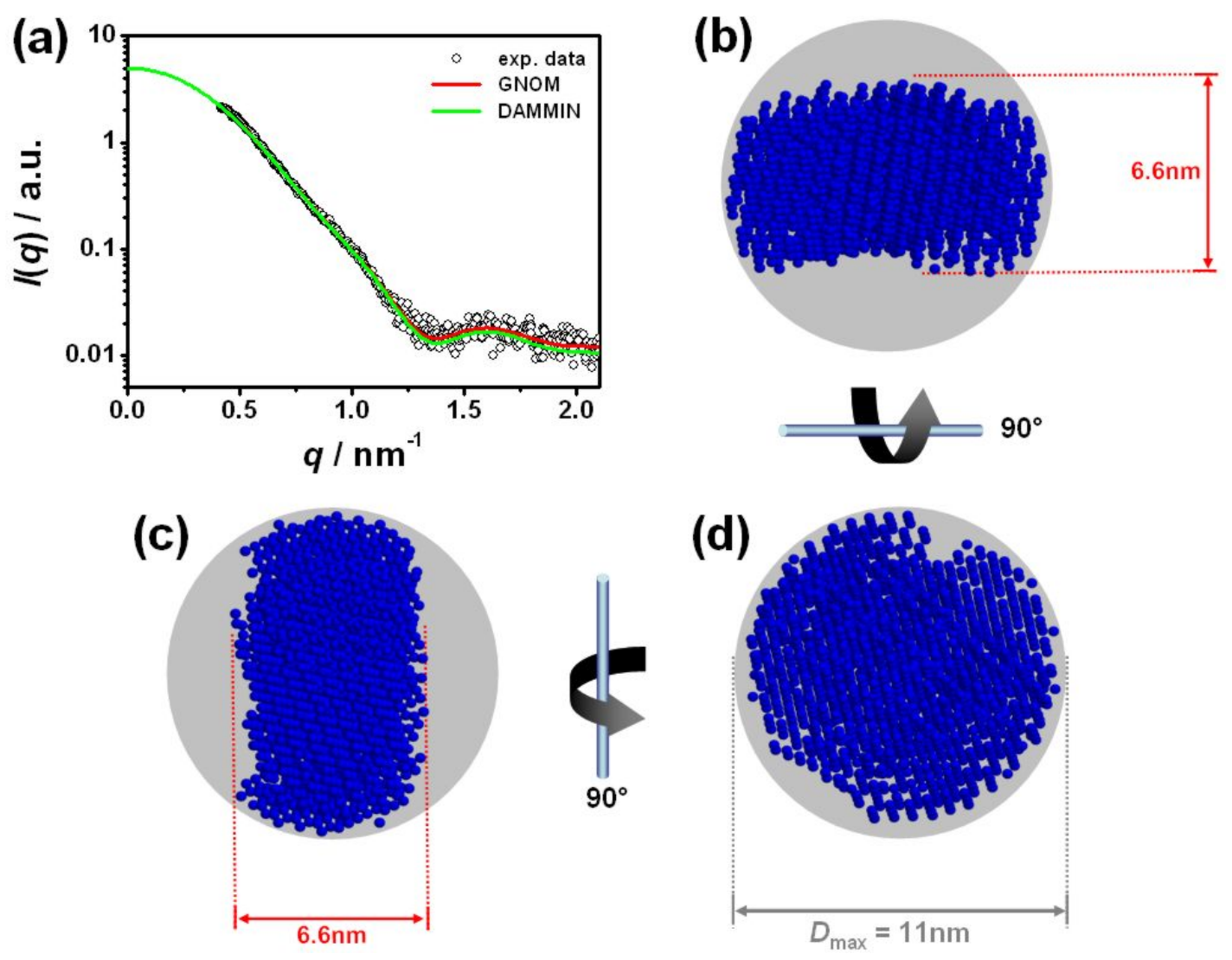

Figure 8-26: DAMMIN model of PAMAM 6/DNA scattering entities at pH $=\mathbf{5 . 5}$.

Assigning again a $d_{\mathrm{DNA}}=2.0 \mathrm{~nm}$ hard-core diameter to the DNA, ${ }^{53}$ these results suggest a PAMAM 6 dendrimer radius of

$$
R_{P 6} \approx \frac{a_{D A M M I N}-2 \cdot d_{D N A}}{2} \approx \frac{b_{D A M M I N}}{2}=3.3 \mathrm{~nm}
$$

Consistent with observations of PAMAM 6/DNA entities at $\mathrm{pH}=8.5$ in chapter 8.2.3, this value is slightly smaller than the radius of PAMAM 6 dendrimers $\left(R_{\mathrm{P} 6}(\mathrm{pH}=5.5)=3.55 \mathrm{~nm}\right)$ determined in chapter 6.4. Therefore, one can conclude that independent of $\mathrm{pH}$ conditions dendrimers seem to shrink upon the interaction with the oppositely charged DNA.

In Figure 8-27, the obtained averaged bead model of PAMAM 6/DNA scattering entities at low $\mathrm{pH}$ conditions is compared to the structure of NCPs obtained from crystallographic X-ray diffraction data (protein data base file leqz.pdb). ${ }^{236}$ Analogue to Figure 8-11, Figure 8-27 shows the DAMMIN model of PAMAM 6/DNA scattering entities (a), the structure of NCPs obtained from the crystallographic data (b), and a superposition of both (c). Consistent with results obtained from the corresponding pair 
(a)
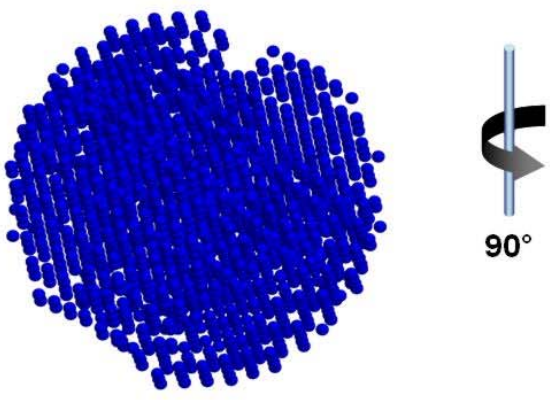

(b)

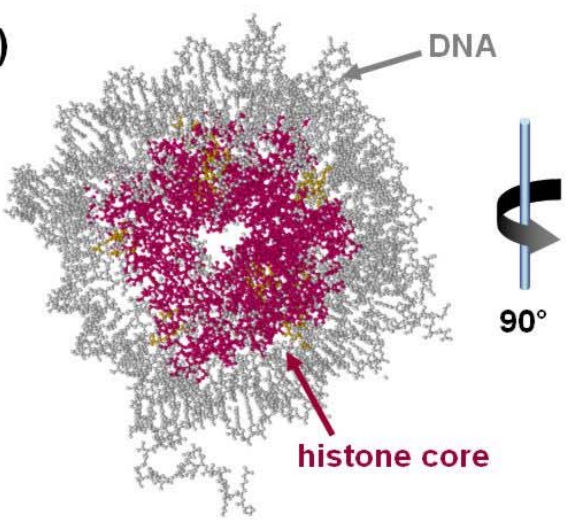

(c)

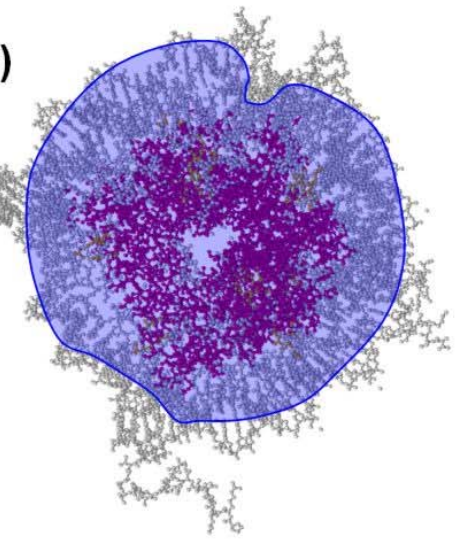

(d)

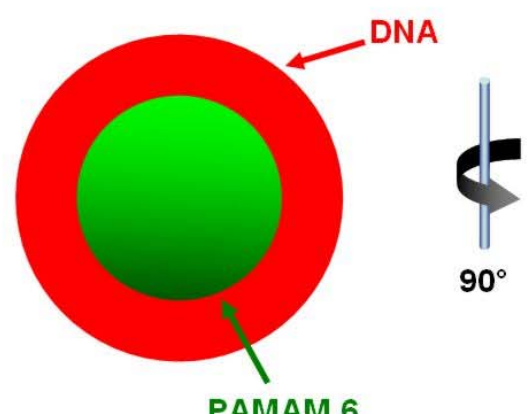

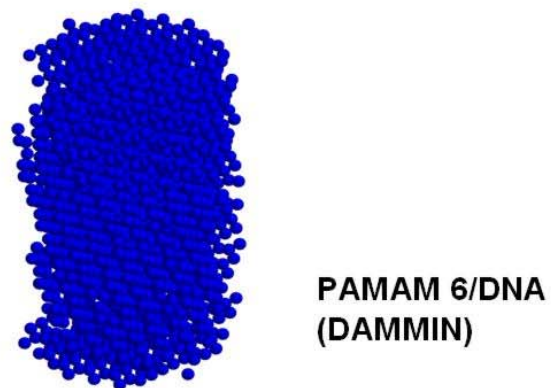
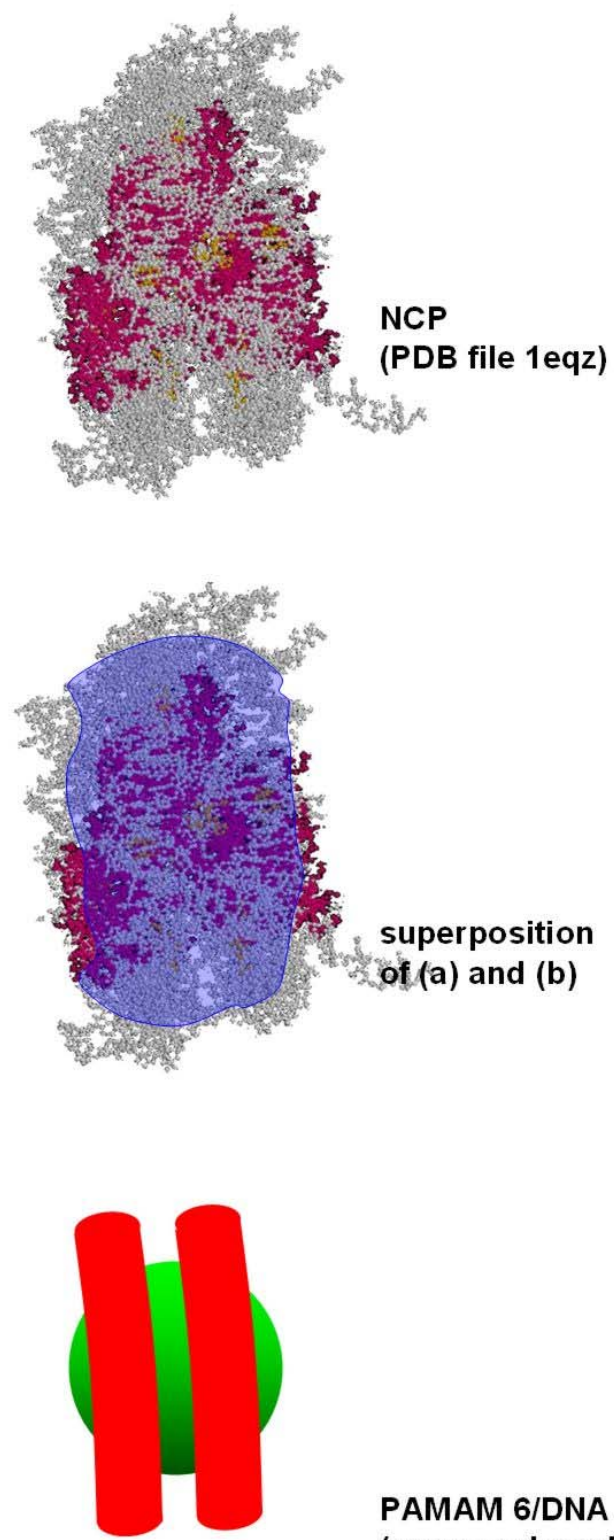

PAMAM 6/DNA (proposed model)

Figure 8-27: Comparison of PAMAM 6/DNA scattering entities and NCPs. (a) PAMAM 6/DNA, (b) NCP, (c) superposition of (a) and (b). (d) Schematic representation of the proposed structure of PAMAM 6/DNA entities. 


\begin{tabular}{|c|c|c|}
\hline & $\mathrm{pH}=5.5$ & $\mathrm{pH}=8.5$ \\
\hline \multicolumn{3}{|c|}{ PAMAM 6} \\
\hline radius in solution $R_{\mathrm{P} 6}$ & $3.55 \mathrm{~nm}$ & $3.04 \mathrm{~nm}$ \\
\hline radius in complex $R_{\mathrm{P} 6 \mathrm{C}}$ & $3.3 \mathrm{~nm}$ & $2.8 \mathrm{~nm}$ \\
\hline total charge $Q_{\mathrm{P} 6}$ & $405 \mathrm{e}^{+}$ & $160 \mathrm{e}^{+}$ \\
\hline contribution of primary amino groups $Q_{\mathrm{I}}$ & $256 \mathrm{e}^{+}$ & $160 \mathrm{e}^{+}$ \\
\hline contribution of tertiary amino groups $Q_{\text {III }}$ & $149 \mathrm{e}^{+}$ & $0 \mathrm{e}^{+}$ \\
\hline surface charge density $\Sigma_{\mathrm{P} 6}$ & $1.6-1.9 \mathrm{e}^{+} / \mathrm{nm}^{2}$ & $1.46 \mathrm{e}^{+} / \mathrm{nm}^{2}$ \\
\hline \multicolumn{3}{|l|}{ DNA } \\
\hline absorbed amount of $b p$ & $135 \mathrm{bp}$ & $35 \mathrm{bp}$ \\
\hline absorbed length $L_{\mathrm{a}}$ & $46 \mathrm{~nm}$ & $12 \mathrm{~nm}$ \\
\hline diameter $d_{\mathrm{DNA}}$ & \multicolumn{2}{|c|}{$2 \mathrm{~nm}$} \\
\hline line charge density $\tau_{\mathrm{DNA}}$ & \multicolumn{2}{|c|}{$6 \mathrm{e}^{-/ \mathrm{nm}}$} \\
\hline persistence length $L_{\mathrm{P}}{ }^{249}$ & \multicolumn{2}{|c|}{$50 \mathrm{~nm}$} \\
\hline covered dendrimer surface area $A_{\mathrm{P} 6 \mathrm{C}}$ & $92 \mathrm{~nm}^{2}$ & $24 \mathrm{~nm}^{2}$ \\
\hline absorbed charge $Q_{\mathrm{DNA}}$ & $270 \mathrm{e}^{-}$ & $70 \mathrm{e}^{-}$ \\
\hline local overcharging $\left(Q_{\mathrm{DNA}} / A_{\mathrm{P} 6 \mathrm{C}}-\Sigma_{\mathrm{P} 6}\right)$ & $1.0-1.3 \mathrm{e}^{-} / \mathrm{nm}^{2}$ & $1.46 \mathrm{e}^{-/} / \mathrm{nm}^{2}$ \\
\hline bending energy $E_{\mathrm{b}}$ & $62 k T$ & $22 k T$ \\
\hline
\end{tabular}

Table 8-1: Characteristics of PAMAM 6 dendrimers and DNA at $\mathrm{pH}=5.5$ and pH $=8.5$.

distance distribution functions $p(r)$, the similarity in size and shape of PAMAM 6/DNA and NCP is striking.

Based on the derived bead model, a schematic representation of the proposed structure of the PAMAM 6/DNA scattering entities is given in Figure 8-27d. In NCPs, a total of 146 base pairs are wrapped around the histone core in approximately 1.7 turns. Figure 8-27c suggests that a similar amount of DNA base pairs is wrapped around PAMAM 6. Therefore, the DNA length associated to PAMAM 6 increases significantly with reduction of $\mathrm{pH}$. Contrary to high $\mathrm{pH}$ conditions, the dendrimer seems to be completely encompassed by the DNA. Principle parameters derived from the bead model are listed in Table 8-1. For comparison, corresponding values obtained at $\mathrm{pH}=8.5$ are given.

At $\mathrm{pH}=8.5$, attractive electrostatic interactions of the two components are not strong enough to completely overcome the elastic counterbalances and to induce a complete DNA wrapping. As a consequence, the charge density of $\Sigma=1.46 \mathrm{e}^{+} / \mathrm{nm}$ results in an adsorption of an approximately $12 \mathrm{~nm}$ long stretch of DNA. A charge density of about 
$1.6-1.9 \mathrm{e}^{+} / \mathrm{nm}$ at $\mathrm{pH}=5.5$ is needed in order to enable a complete wrapping of the DNA in approximately 1.7 turns corresponding to $46 \mathrm{~nm}$. Therefore, the presented results show experimentally that a wrapping transition occurs from an adsorption of a finite length of DNA to a full wrapping with increasing the valence of the spherical macroion. This is in good agreement with theoretical studies on the interaction of a spherical macroion and a linear, oppositely charged polyelectrolyte using the linearized Poisson-Boltzmann theory. ${ }^{7,243,247}$

For a semi-flexible polyelectrolyte with a certain bending rigidity and therefore a finite persistence length $L_{\mathrm{P}}$, the chain bending upon adsorption onto a sphere surface disfavors wrapping. Two effects are included in $L_{\mathrm{P}}$, namely the mechanical bending rigidity of the uncharged chain and the electrostatic repulsions of like-charged chain segments. ${ }^{247,248}$ With the parameters in Table 8-1 at hands, it is possible to estimate the bending penalty, which has to be overcome by adsorption energies in order to enable a wrapping of the determined stretches of DNA around the sphere. The DNA bending energy $E_{\mathrm{b}}$ around a PAMAM 6 sphere can be considered in terms of $k T$ according to following equation: ${ }^{248}$

$$
\frac{E_{b}}{k T}=\frac{L_{P} L_{a}}{2\left(R_{P 6 C}+d_{D N A} / 2\right)^{2}} .
$$

Variables in equation 8-8 are chosen to be consistent to those defined in Table 8-1. $R_{\mathrm{P} 6 \mathrm{C}}+d_{\mathrm{DNA}} / 2$ estimates the curvature of the DNA. Obtained results show that an energy gain upon adsorption of approximately $62 k T$ is needed in order to achieve a complete DNA wrapping in about two turns around the dendrimer. However, obtained values represent a lower estimate, since e.g. contributions due to twist and torsion of the polyelectrolyte chain are neglected.

At $\mathrm{pH}=5.5$, the large amount of DNA adsorbed on the dendrimers leads to a strong, overall overcharging of the spherical cation: The overall charge of the DNA adsorbed on the PAMAM 6 surface exceeds the initial charge of the dendrimer, so that the net charge of the entity changes sign. The phenomenon of overcharging of spherical macroions complexed with oppositely charged, linear polyelectrolytes is well known in literature. $^{240,251}$ In the X-ray patterns, it is reflected by the strong inter-particle repulsions, which are observed at all studied conditions as pronounced downturns in the low $q$ region. Consequently, these inter-particle repulsions prevent the organization of long-range ordered complex structures. It is important to point out, that at $\mathrm{pH}=8.5$ overcharging effects are also present. However, they are only locally encountered, but are more pronounced (Table 8-1). 


\subsection{Relevance and future directions}

Compared to histone cores, which are of rather cylindrical shape and possess a very rough surface with specific binding sites for the DNA, the spherical and uniformly charged PAMAM 6 dendrimers represent only a very rough approximation of the real structure. Despite these facts, the cylindrically shaped, fully wrapped conformation of PAMAM 6/DNA entities at $\mathrm{pH}=5.5$ is strikingly similar in shape and size to the structure of nucleosome core particles (NCPs). Therefore, the PAMAM 6/DNA system clearly represents the simplest possible model system - no additional organic components no added salt, only monovalent ions, monodisperse, spherical cations with controllable charge densities -, which still captures the characteristic features of histone core/DNA interaction. Consequently, one may conclude that at least for a simple replication of the beads-on-a-string structure without paying attention to DNA functionality, a specific arrangement of charged patches on the histone octamer is not required.

To date, the next step of chromatin organization, the $30 \mathrm{~nm}$ fiber, is widely unclear. The exact architecture is still unknown as are the forces that drive the formation of the $30 \mathrm{~nm}$ fiber. In order to elucidate its structure, several researchers have performed in vitro studies of the NCP organization in crystals and mesophases. ${ }^{22,}{ }^{218-220}$ In this context, it is important to accentuate the fact that - contrary to NCP crystals - in PAMAM 6/DNA complexes, connecting (linker-)DNA strands are still present in the structure. Therefore, the PAMAM 6/DNA system represents an ideal starting point towards an experimental mimicking of the $30 \mathrm{~nm}$ fibre, e.g. by varying salt concentrations and/or adding linker-histones.

A first step towards an understanding of the organization of the $30 \mathrm{~nm}$ fiber is to understand the properties of complexes in solution. Therefore, one has to also consider the interaction between different PAMAM 6/DNA entities. These interactions are in particular relevant at small salt concentrations, where the screening length is large. ${ }^{247}$ Consequently, results presented here yield information about both the nature of PAMAM 6/DNA entities and the organization of formed complexes. Therefore, in Figure 8-28 the schematic representation of the charge density dependent evolution of PAMAM 6/DNA conformations is contrasted to that of corresponding complex organizations (chapter 8.4.1). Since analyzing the complex interplay involved in the organization of a beads-on-a-string structure is far beyond the possibilities of simulations, experimental results obtained from simple model systems are expected to be an important contribution to the field. 


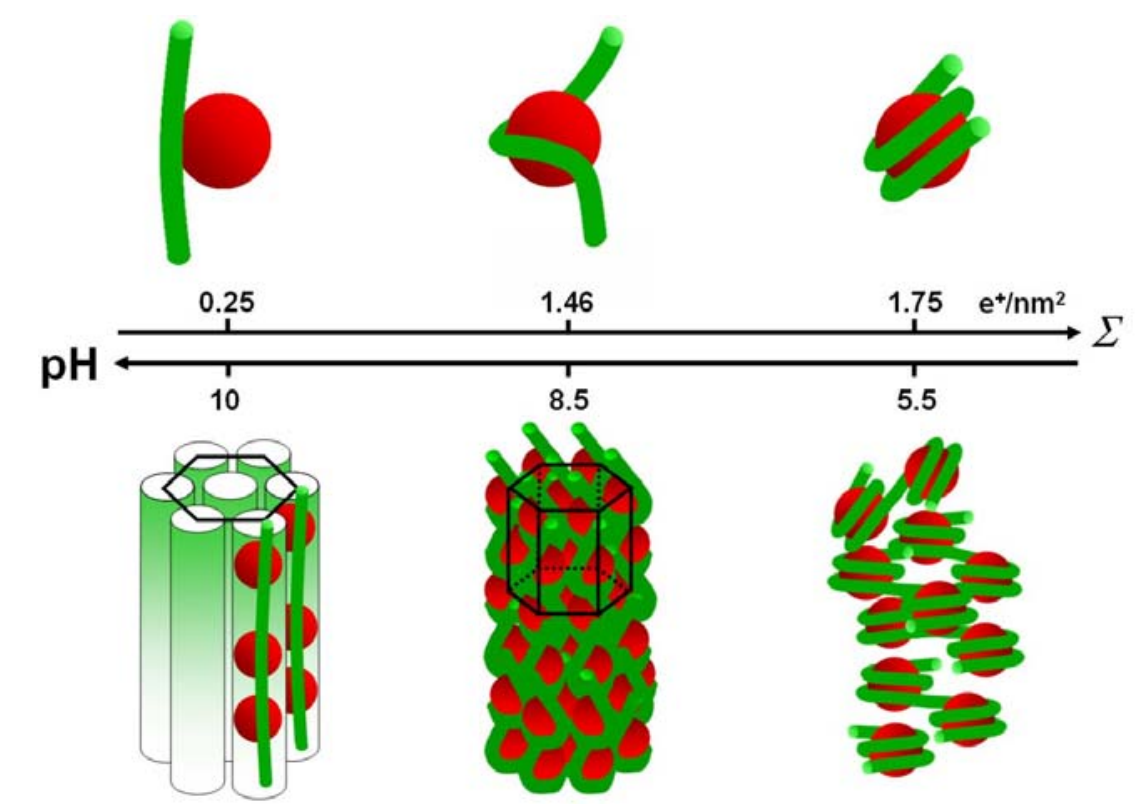

Figure 8-28: Schematic representation of the different conformations of PAMAM 6/DNA entities (top) and complex structures (bottom) in dependence of the $\mathrm{pH}$ controlled dendrimer surface charge density $\Sigma$.

As a consequence of the DNA wrapping, an increased amount of DNA is directly adsorbed on the dendrimer surface. Accordingly, a higher degree of DNA compaction can be achieved. Tentatively, such more compact dendrimer/DNA complexes may more readily penetrate cell membranes and more easily enter the cell cytoplasm. ${ }^{242}$ This would provide an explanation for the enhanced gene transfection efficiency of larger dendrimers compared to smaller ones. ${ }^{252}$ Moreover, the larger fraction of tightly bound (adsorbed) DNA regions reduces their accessibility to nuclease digestion and enhance DNA protection. Therefore, the wrapping scenario provides a plausible rationale for the observed increased stability of DNA complexes with higher generation dendrimers against nuclease digestion. ${ }^{225}$ Following this line of argumentation, low $\mathrm{pH}$ conditions may be better suited for the purpose of DNA delivery inside cells since a larger fraction of DNA is wrapped around the dendrimer and therefore protected.

\subsection{Conclusion}

Results presented in this chapter clearly show that PAMAM 6 dendrimers, which possess dimensions and charges comparable to those of the histone core, are able to mimic histones. Despite its high degree of simplicity, the PAMAM 6/DNA system is able to reproduce characteristic structural features of chromatin organization in vivo. 
The cylindrically shaped, fully wrapped conformation of PAMAM 6/DNA scattering entities at low $\mathrm{pH}$ is remarkably similar in shape and size to the structure of NCPs. In this sense, PAMAM 6/DNA entities can be view as artificial nucleosome core particles. Controlling only the $\mathrm{pH}$ dependent dendrimer valence, it is possible to tune the interaction of PAMAM 6 and DNA. A wrapping transition from an adsorption of a finite length of DNA to a full wrapping has been shown to take place with increasing the valence of the spherical macroion. Just as in the case of protein folding, studying PAMAM 6/DNA complex formation in microflow reveals that the condensation process exhibits multistage dynamics. Consistently, PAMAM 6/DNA interaction kinetics are no longer diffusion limited and particularly slow. At low $\mathrm{pH}$, the significant overcharging of PAMAM 6 by the adsorbed DNA prevents establishing of long-range ordered complex structures. Reducing the dendrimer surface charge density, however, leads to the formation of highly ordered 3D hexagonal lattice structures. The experiments presented in this chapter lay a foundation for future studies towards an experimental mimicking of the $30 \mathrm{~nm}$ fiber. Therefore, the simple PAMAM 6/DNA model-system is expected to be a subject of considrable biophysical interest. 


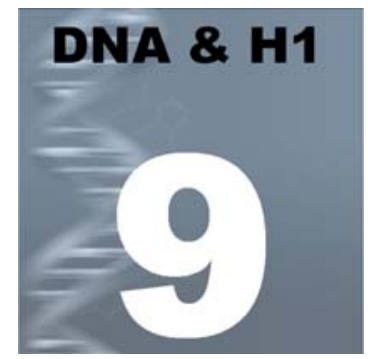

\section{DNA COMPACTION: LINKER-HISTONES H1}

Histones are the major protein component of chromatin. Structurally, one distinguishes between two main categories of histones: "core" and "linker" histones. Core histones (histones $\mathrm{H} 2 \mathrm{~A}, \mathrm{H} 2 \mathrm{~B}, \mathrm{H} 3$, and $\mathrm{H} 4$ ) are arranged as a globular octameric core. Around these $146-180 \mathrm{bp}$ of DNA are wrapped in approximately $13 / 4$ left-handed super-helical turns forming nucleosomal core particles, which are connected by a variable stretch of linker-DNA. ${ }^{24,25}$ The major function of histones of the $\mathrm{H} 1$ family is to extensively interact with linker-DNA. Hence, they are known as linker-histones. Despite the common name, linker-histones are not related to core histones. In eukaryotic cells, binding of linker-histones to the linker-DNA facilitates the shift of chromatin structure towards more condensed, higher order forms (i.e. the $30 \mathrm{~nm}$ chromatin fiber). ${ }^{24}$ These findings suggest that linker-histones are of central importance in genome organization and regulation. Hence, numerous studies of the interaction of linker-histones with DNA as well as of the interaction of linker-histones with nucleosomal core particles have been reported. In addition to the endless primary research literature, a few tens of review articles have been published on the subject in the last decade (e.g. ${ }^{20,23,25,216,253-}$ ${ }^{263}$ ). It is perhaps a measure of the complexity of the problem that titles of several reviews end with a question mark (e.g. ${ }^{259,264-266}$ ).

Despite extensive studies, most basic questions such as linker-histone location and function are still unanswered. Most surprisingly, so far there are no small angle scattering studies available in literature probing linker-histone/DNA interaction dynamics and structure formation on relevant molecular length scales. In this chapter, the combination of microfluidics and small angle X-ray microdiffraction is used to gain 
new insights in linker-histone/DNA interaction dynamics and structure formation. In particular, owing to the laminar flow conditions inside microchannels, additional possibility to investigate the interaction process in a time-resolved manner is provided. Moreover, the H1/DNA system is used to demonstrate some basic principles of microfluidics employing complex fluids and to show in detail the significant advantages for the characterization of complex fluids arising from the combination of $\mathrm{X}$-ray microdiffraction and microfluidics.

\subsection{Structure and function of linker-histones}

Linker-histones are some of the most abundant proteins in cellular nuclei. The linkerhistone family in higher eukaryotes is a heterogeneous family of highly tissue-specific, basic proteins such as the histone $\mathrm{H} 5$ from nucleated erythrocytes of birds ${ }^{267}$ and sperm PL-I proteins ${ }^{268}$ which exhibit significant variations in sequence. However, most of eukaryotic histones $\mathrm{H} 1$ share a similar tripartite structure consisting of a globular domain flanked by relatively unstructured lysine-rich domains, a shorter aminoterminal one and a longer, carboxyl-terminal one. ${ }^{260}$ Henceforth, these are referred to as N-tail and C-tail (Figure 9-1a).

The structure of the globular domain (diameter $2.9 \mathrm{~nm}$ ), ${ }^{269}$ which is highly conserved throughout all types of eukaryotic linker-histones, ${ }^{260}$ has been solved by X-ray crystallography $^{270}$ and NMR studies ${ }^{271}$ identifying it to be member of the winged helix class of DNA-binding domains exhibiting three $\alpha$-helices (Figure 9-1a). The globular domain is the only $\mathrm{H} 1$ domain that is folded in solution. ${ }^{272}$ However, in contrast to other members of this class of proteins, the globular domain of linker-histones contains a distinct, additional cluster of positively-charged amino acids. These form a second DNA-binding location, on a side of the protein opposite to the primary DNA-binding site. ${ }^{273}$ Linker-histones are known to attach specifically close to the entry and exit sites of linker-DNA on the nucleosome core bringing together the two linker DNA segments. ${ }^{257,264,274}$ Studies have shown that the globular domain is responsible for this structure-specific recognition, ${ }^{275}$ most likely provided by the specific arrangement of the two binding sites. ${ }^{276}$ However, little is known about the specific details of this important interaction, and several models for the exact positioning of linker-histones on the nucleosomal core particle have been proposed. ${ }^{274,277-279}$

Contrary to the evolutionary well conserved winged helix motif of the globular domain, both linker-histone tails are extremely heterogeneous in length as well as amino acid 

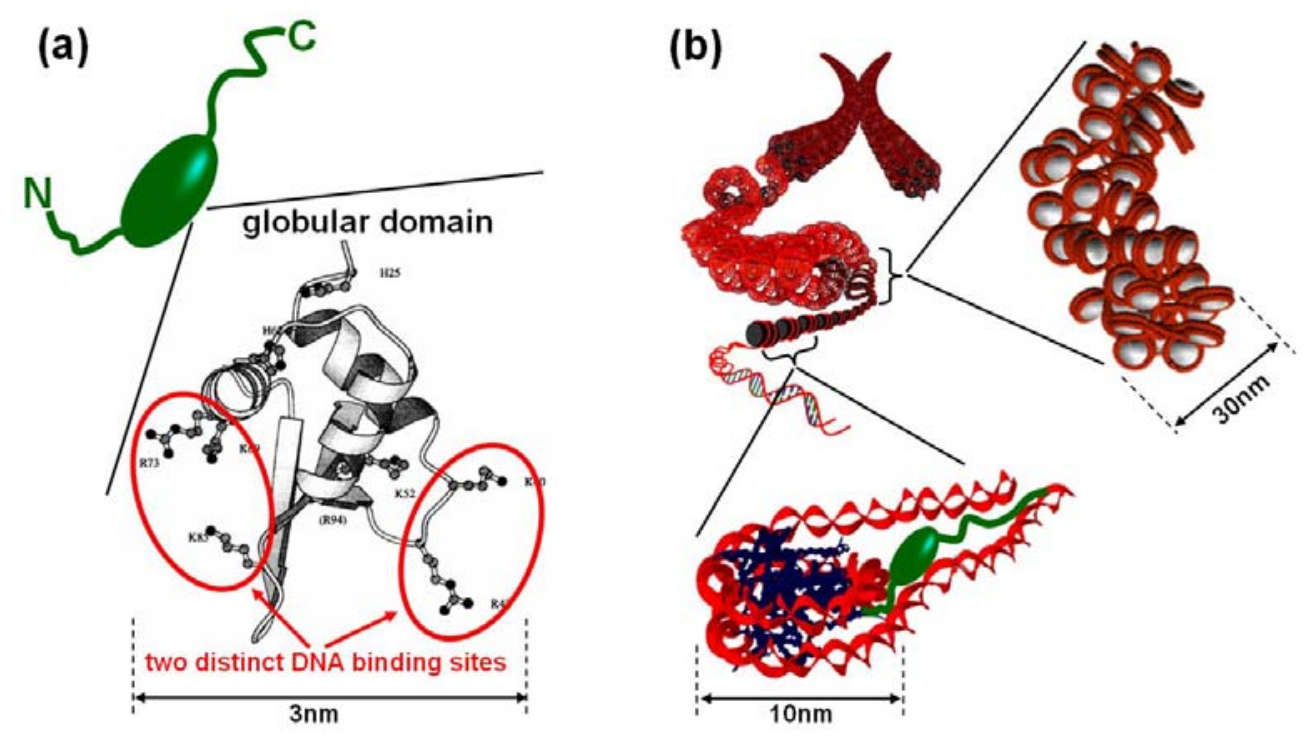

Figure 9-1: (a) Schematic representation of $\mathrm{H1}$ structure showing the globular domain flanked by the $\mathrm{N}$ - and the $\mathrm{C}$-tail. Additionally, a ribbon model of the 3D structure of the globular domain is given. ${ }^{270}$ (b) Linker-histone H1/linker-DNA interactions (bottom) mediate chromatin transition into the $30 \mathrm{~nm}$ fiber (right).

composition. The absence of an $\alpha$-helical structure of the tails in aqueous solutions is attributed to electrostatic repulsion between highly charged lysine side chains. However, it has been shown that both tails of several linker-histone H1 subtypes exhibit structure when exposed to helix-stabilizer (e.g. trifluoroethanol, $\mathrm{HClO}_{4}$ ) and attain optimum secondary and hence tertiary structure upon interaction with DNA. ${ }^{280-286}$

Despite positioning along the nucleosomal core particle, it is not the globular domain but rather the highly charged, C-tail that imparts to linker-histones their unique ability to bind to linker-DNA. ${ }^{274,287,288}$ Thus, the presence of the C-tail appears to be essential for processes of chromatin folding and DNA compaction. ${ }^{255,288,289}$ In vivo, the absence of this structural feature leads to greatly reduced chromatin binding. ${ }^{290}$

The functional and structural roles of the N-tail are still widely unclear. ${ }^{288}$ In many H1 subtypes, the N-tail presents two distinct subregions. ${ }^{291}$ The distal half is devoid of basic residues, whereas the half immediately adjacent to the globular domain contains a large amount of basic groups. The majority of studies available in literature agree that $\mathrm{N}$-tails bind to DNA rather poorly. ${ }^{288,}{ }^{292}$ Even though the N-tail does not seem to be essential for chromatin folding, this domain may be involved in the exact positioning and anchoring of the globular domain. ${ }^{288}$ Furthermore, there is a controversy in literature about in how far N-tails may mediate head-to-tail interactions of neighboring 
linker-histones leading to a preferential $\mathrm{H} 1-\mathrm{H} 1$ association and therefore cooperative binding to DNA. ${ }^{293}$

It has long been believed that the function of linker-histones is primary an architectural one helping to create higher order structures of the chromatin fiber. ${ }^{24}$ Although a chromatin fiber lacking linker-histones is able to fold to a certain extent, ${ }^{263}$ there is abundant evidence that the highly ordered chromatin compaction of the $30 \mathrm{~nm}$ fiber is only attained in the presence of linker-histones. ${ }^{20,24,264}$ From changes in chromatin conformation observed at different salt concentrations, the mechanism of chromatin compaction is assumed to be primarily electrostatic. ${ }^{19,294}$ Contributing to the free energy of chromatin folding, linker-histones help to select a specific folding state from among the set of compact states reached in its absence. ${ }^{253}$

Moreover, being involved in the build-up of the 30nm fiber, which presumably limits the access of the transcriptional machinery, linker-histones may function as generalized repressors. Consistently, transcriptionally active chromatin is typically depleted in linker-histones compared with inactive chromatin. ${ }^{295-297}$ However, experiments performed in vivo indicate that linker-histones may regulate transcription at a finer level by contributing to chromatin architecture and by participating in complexes that either activate or repress specific genes. ${ }^{264,298-301}$ Besides their architectural and generegulatory functions, recent reports indicate that linker-histones are involved in much more fundamental cellular processes. Linker-histones have been shown to play a prominent role in cell ageing, ${ }^{302}$ DNA repair, ${ }^{303}$ apoptosis, ${ }^{304}$ and muscle development. ${ }^{305}$ This raises the possibility that biological functions of linker-histones are more varied than previously imagined. However, all of these functions are believed to be highly depending on H1/DNA interaction mechanisms.

Histone $\mathrm{H} 1$ has also been shown to condense DNA in vitro. ${ }^{287,306-314}$ It has been argued that H1/DNA complexes are a good model system for studying aspects of the interaction of $\mathrm{H} 1$ with chromatin. The observation, that salt concentration effects are remarkable in H1/DNA reactions, is suggestive of a nonspecific binding, mainly controlled by electrostatic interactions analog to processes in vivo. ${ }^{306,314}$ H1/DNA complexes observed by electron microscopy posses a unique tramtrack-like conformation consisting of two DNA strands bridged by an array of protein molecules and multiples of these. ${ }^{308}$ This reflects the existence of the two distinct DNA binding sites on the globular domain, apparently mirroring the situation at the $\mathrm{H} 1$ binding site on the nucleosome. Although the molecular basis of H1/DNA condensation is not yet clear, it is generally believed that understanding the way in which these proteins bind DNA can help clarifying the still unresolved problem of how $\mathrm{H} 1$ bind to nucleosomal structures in chromatin. ${ }^{24}$. From a more general point of view, the H1/DNA system can be regarded as a model for non-specific DNA-protein interactions. 
(a)
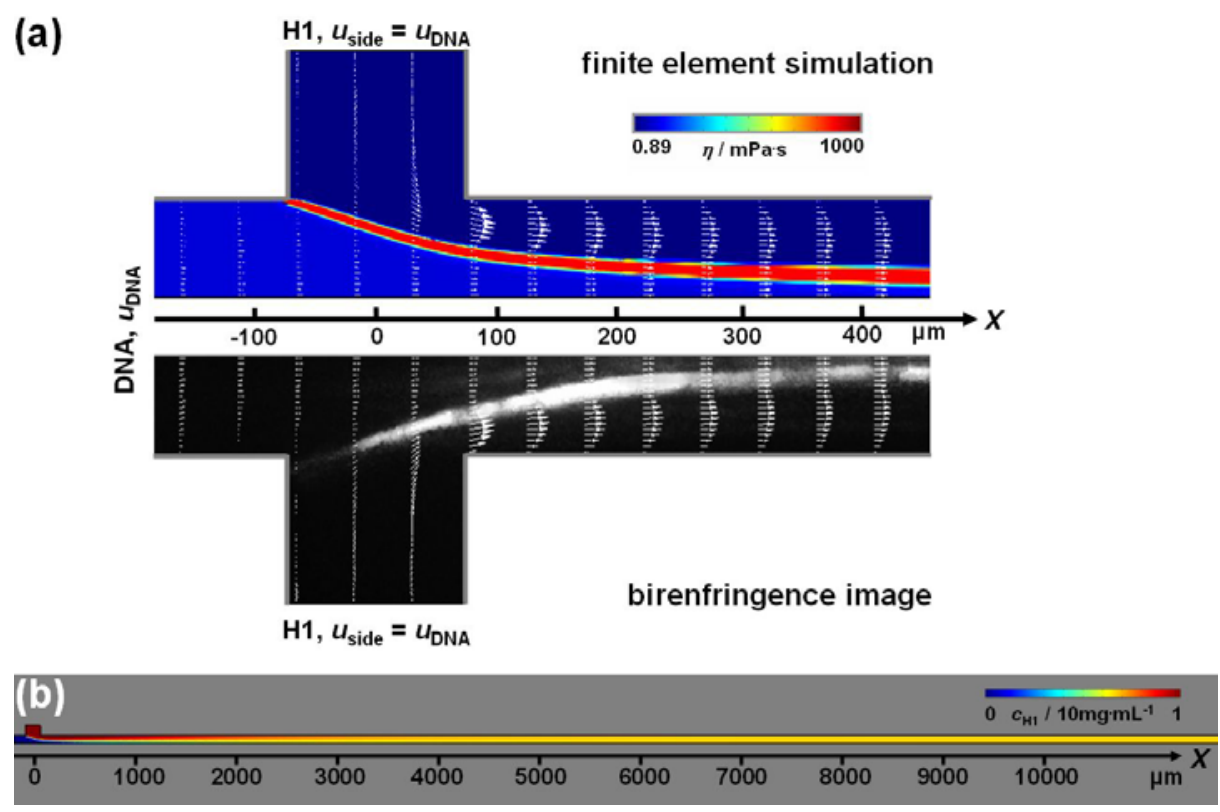

Figure 9-2: Real-time monitoring of linker-histone H1 induced DNA compaction in a hydrodynamic focusing device. (a) Simulation results (top) and birefringence data (bottom) close to the confluence region are contrasted $\left(u_{\mathrm{DNA}}=600 \mu \mathrm{m} \cdot \mathrm{s}^{-1}\right)$. The product of the complexation reaction appears in the diffusion cone of side and main stream components due to its highly increased viscosity. (b) Simulated H1 concentration profile of the whole device $(x=-200-12000 \mu \mathrm{m})$.

In the following, the interaction of DNA with calf thymus linker-histone H1 (isolated lysine rich fraction, ${ }^{315}$ Sigma-Aldrich $\mathrm{GmbH}$, Taufkirchen, Germany) with a molecular weight of $M_{\mathrm{w}}=21.5 \mathrm{kDa}$ and 55 positive charges at physiological $\mathrm{pH}$ conditions ${ }^{314}$ is studied in hydrodynamic focusing devices. X-ray data are recorded at the beamline ID10b at the ESRF (chapter 2).

\subsection{Monitoring $\mathrm{H} 1$ induced DNA compaction in microflow}

Polarized light microscopy has widely been used to study DNA complexes, which are known to be highly birefringent. ${ }^{53,54,214}$ The birefringence signal is increased upon self-assembly of DNA complexes under conditions of alignment and elongation as can be superimposed by using microflow devices. Consistently, combining microfluidics with birefringence microscopy provides a fast and easy access to direct imaging of $\mathrm{H} 1$ induced DNA compaction. The hydrodynamic focusing device used here consists of 
two perpendicularly crossed microchannels having a depth of $300 \mu \mathrm{m}$ and a width of $150 \mu \mathrm{m}$. A semi-diluted aqueous DNA solution with a concentration of $c_{\text {DNA }}=2.5 \mathrm{mg}^{-1} \mathrm{ml}^{-1}$ is injected in the reaction channel and hydrodynamically focused by two side streams of aqueous $\mathrm{H} 1$ solutions with a concentration of $c_{\mathrm{H} 1}=10 \mathrm{mg} \mathrm{mL}^{-1}$. Data are acquired at three different flow velocities $u_{\mathrm{DNA}}=60,150$ and $600 \mu \mathrm{m}^{-1}{ }^{-1}$. The flow velocities in the side channels are varied such that a flow velocity ratio $u_{\text {side }} / u_{\text {DNA }}=1$ is maintained. Hydrodynamic focusing experiments are performed at physiological pH. According to the discussion in chapter 5.3.1 and 7.1.2, finite element simulations of physical conditions inside the microchannels are performed and compared to the experiment in order to elucidate the experimental situation.

For each flow velocity, two finite element simulations are performed. In order to accurately determine relevant fit parameters, physical conditions in the microchannel device are first simulated with high precision (i.e. high number of finite elements) for a close-up region around the confluence area $(x=-200-500 \mu \mathrm{m})$. The viscosity of the formed H1/DNA assemblies' $\eta_{\text {complex }}$ and the diffusion constant $D_{\mathrm{H} 1}$ are used to match the experimentally recorded shape of the hydrodynamically focused DNA stream and of the formed H1/DNA assemblies. All other parameters such as channel geometry, flow rates, and the viscosity of the DNA solution are known. Additional details on the finite element simulations are given in chapter 5.3.1 and 7.1.2. In order to describe physical conditions at positions further down the reaction channel, a second simulation extending over the whole length of the device $(x=-200-12000 \mu \mathrm{m})$ has been performed using fit parameter values determined in the first set of simulations.

In Figure 9-2a, simulation data from the first set of simulations are exemplarily shown for $u_{\mathrm{DNA}}=600 \mu \mathrm{m}^{-1}$ and contrasted to corresponding experimental results. Subsequently to the intersection, H1 molecules diffusive into the DNA stream and the H1/DNA interaction can be observed along the outlet channel. The optically birefringent pattern reflects that the DNA chains in the complexes are orientationally ordered due to the superimposed flow. For direct comparison with experimental results, the modeled velocity profile (arrow plot) in the hydrodynamic focusing device is overlaid to the recorded birefringence image (bottom). In simulations, H1/DNA complexes can be best visualized by utilizing the fact that a strong increase in local viscosity is connected to the complexation reaction. According to the discussion in chapter 7.1.2, insignificant deviations of simulation results from the experimentally recorded shape are observed in the crossing area where the center stream is slightly bulged into the side channels. These deviations result from the fact that simulations are performed in two dimensions whereas the experimental system is affected by additional walls at the top and the bottom of the device. ${ }^{195}$ 

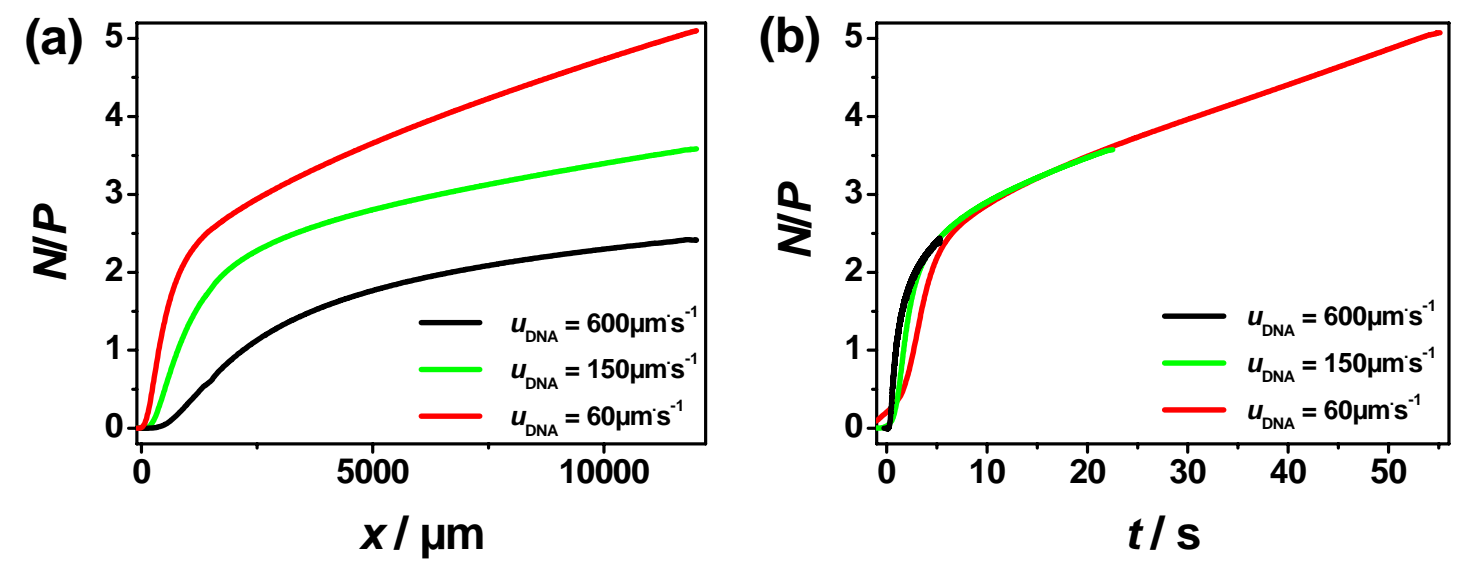

Figure 9-3: Dependence of the $N / P$ ratio on the position $x$ along the outlet channel (a) and the time $t(b)$.

Apart from this detail, experiments and simulations show good agreement. The result of the corresponding simulation over the whole range of the device is given in Figure 9-2b. Adjusting simulations to match experimental results yields information related to the viscosity of H1/DNA condensates and the diffusion constant of linker-histones. Independent of the flow velocity, throughout all simulations the viscosity of H1/DNA complexes is fitted to $\eta_{\text {complex }} \approx 3 \cdot 10^{3} \cdot \eta_{\text {water }}=2.7 \mathrm{~Pa}$ s. This result is on the same order of magnitude as results known from other polymer hydrogels. ${ }^{195-197}$ H1 proteins are successively diffusing through these highly viscous regions. A rather high diffusion constant of $D_{\mathrm{H} 1} \approx 2 \cdot 10^{-10} \mathrm{~m}^{2 \cdot} \mathrm{s}^{-1}$ is found, which is close to the result one obtains from the Stokes-Einstein relation ( $r_{\text {globular domain }} \approx 1.5 \mathrm{~nm}, \quad D_{\mathrm{SE}} \approx 1.7 \cdot 10^{-10} \mathrm{~m}^{2} \cdot \mathrm{s}^{-1}$ ) under purely aqueous conditions. The magnitude of $D_{\mathrm{H} 1}$ is directly and significantly affecting calculated local H1 concentrations and therefore $N / P$ ratios. The fact that a highly consistent picture is evolving when combining the huge amount of recorded X-ray data with simulation results allows for a validation of $D_{\mathrm{H} 1}$ as will be discussed in chapter 9.3.1.

From simulations, local experimental conditions such as flow velocities and concentrations can be obtained at each position. In Figure 9-3a, simulated $N / P$ ratios obtained for different flow velocities are given as a function of the position $x$ along the center of the outlet channel $(y=0)$. Using hydrodynamic focusing microdevices, mixing and concentration distributions in the reaction channel can be adjusted by changing main and side channel velocities. Consequently, interaction dynamics and DNA containing mesophase formation - both depending on concentration distributions and therefore on the $N / P$ ratio - can be spatially separated in steady state flow by varying flow velocities and/or observation position along the outlet channel. Experimental conditions are adjusted such that each position corresponds to a different 
complex composition. Flow velocities are varied to result in final charge ratios at the furthest measurable point of the device $(x \approx 12 \mathrm{~mm})$ of $N / P<2.4,3.3$, and 5.1 for $u_{\mathrm{DNA}}=60,150$, and $600 \mu \mathrm{m}^{-1}$, respectively. Local flow velocities can be used to translate positional changes along each streamline into corresponding reaction time coordinates $t$.

Figure 9-3b shows the $t$ dependence of $N / P$. For larger $t, N / P$ ratios collapse onto a master curve. Deviations at initial time states reflect differences in the flow velocity depending strain rate $\dot{\varepsilon}=\partial u / \partial y$, as will be discussed in chapter 9.4. These experimental conditions are utilized to access relevant time states and complex compositions. Moreover, the well defined, purely diffusive mixing of components significantly reduces the likelihood of creating kinetically trapped phases. This is an indispensable characteristic of this study.

\subsection{Analyzing $\mathrm{H} 1$ induced DNA compaction}

\subsubsection{Structure of H1/DNA mesophases}

Spatially resolved small angle X-ray diffraction data are obtained at varying $x$-positions along the main channel for three different initial flow velocities and plotted in terms of the reciprocal vector $q$. The positional accuracy is on the order of the beam size $(20 \mu \mathrm{m})$. Structural information can be obtained from the effective structure factor $S$ which is obtained by revising the scattering intensity $I(q)$ by the normalized intensity of a dilute sample. Qualitatively, peaks of the structure factor at distinct $q$-positions can be interpreted as Bragg reflections from planes of particles separated by a certain mean nearest-neighbor distance $d \sim q^{-1}{ }^{316}$ In Figure 9-4, characteristic 2D diffraction images are exemplarily shown for $u_{\mathrm{DNA}}=150 \mu \mathrm{m}^{-1}$. Plots of X-ray data obtained by radial averaging of the 2D raw images are offset for clarity. Since DNA has a significantly higher electron density than $\mathrm{H} 1$ proteins, the DNA packing dominates the scattering profile. At positions close to the channel intersection, X-ray pattern exhibit two relatively sharp, overlapping Bragg reflections. The decomposition is successfully made by best fitting two Lorentzian functions yielding peak positions $q_{1}$ and $q_{2}$. In Figure 9-4, this is exemplarily shown for $x=100 \mu \mathrm{m}$. 

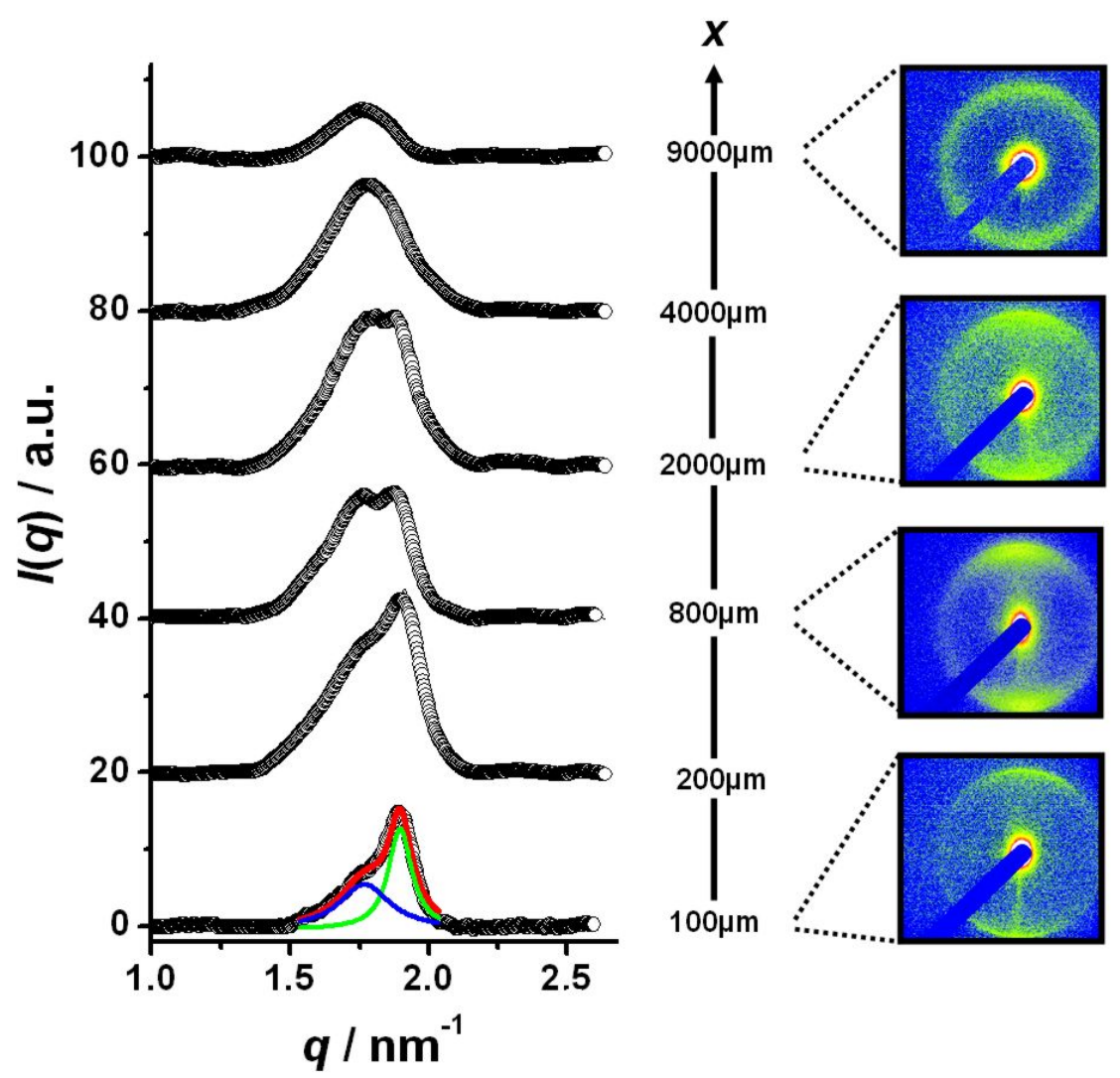

Figure 9-4: Representative 2D X-ray diffraction images (right) obtained at $u_{\mathrm{DNA}}=150 \mu \mathrm{m} \cdot \mathrm{s}^{-1}$ in the middle of the outlet channel $(y=0)$ at different positions $x$ and the extracted, radially averaged $q$-dependence of scattering intensities (left).

In Figure 9-5a, the dependence of the peak positions $q_{1}$ and $q_{2}$ on the position $x$ is given for the data set recorded at $u_{\mathrm{DNA}}=150 \mu \mathrm{m}^{-1}$. In order to elucidate their dependence on time and complex composition, it is reasonable to plot quantities of interest versus $t$ and $N / P$ obtained from simulations. This is shown exemplarily for $q_{1}$ and $q_{2}$ in Figure 9-5b and Figure 9-5c, respectively. Depending on the aim of the performed analysis, in what follows data are plotted versus one of these three variables $(x, t$, and $N / P)$. It is important to stress the fact that using X-ray microdiffraction in combination with microfluidics allows for experimentally accessing H1/DNA structure formation over a wide range of $N / P$ ratios in a minimum of measuring time. Additionally, only small quantities of normally very expensive biomaterials are needed. Plotting quantities extracted from $\mathrm{X}$-ray diffraction data obtained at different flow velocities against $N / P$ allows for collapsing all data onto a master plot showing their complex composition dependence. 

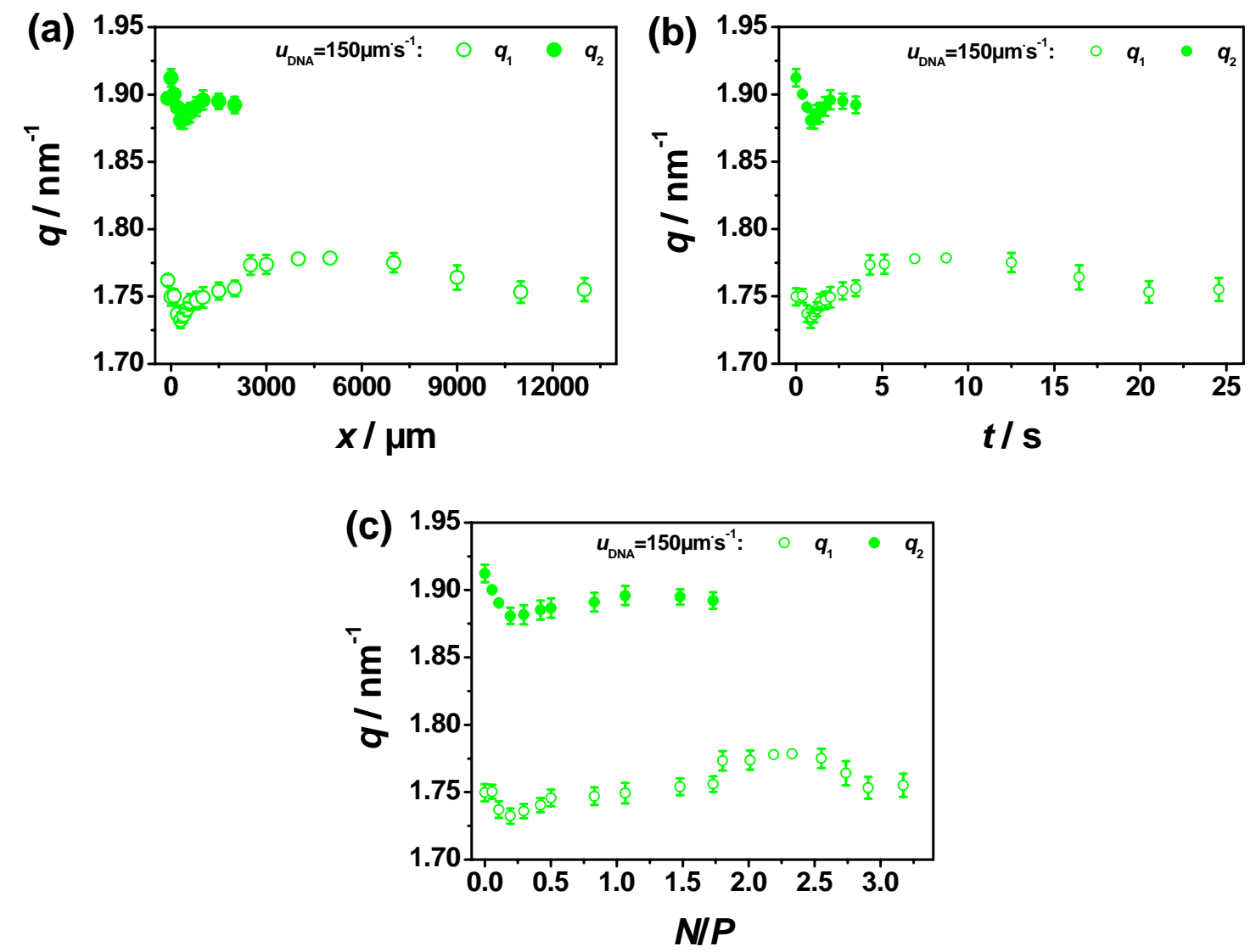

Figure 9-5: Dependence of peak positions $q_{1}$ and $q_{2}$ on the position $x$ along the outlet channel (a), on the reaction time $t(b)$, and on the $N / P$ ratio (c) shown exemplarily for the data set recorded at a flow velocity of $u_{\mathrm{DNA}}=150 \mu \mathrm{m} \cdot \mathrm{s}^{-1}$.

In Figure 9-6, this is demonstrated for peak positions $q_{1}$ (lower curve) and $q_{2}$ (upper curve) measured along the streamline in the center of the reaction channel $(y=0)$. The three data sets obtained at $u_{\mathrm{DNA}}=60,150$, and $600 \mu \mathrm{m}^{-1}{ }^{-1}$ show good agreement with deviations between different data sets of less than $0.01 \mathrm{~nm}^{-1}$. Local $N / P$ ratios are highly depending on the diffusion of $\mathrm{H} 1$ molecules. Accordingly, the fact that data obtained at different velocities are in good agreement and collapse onto master curves validates the determination of $\mathrm{H} 1$ diffusion constant in chapter 9.2. Moreover, it proves the accuracy of the experimental method and the high degree of consistency between experiments and simulations.

At low $N / P$ ratios, peak positions of $q_{1} \approx 1.76 \mathrm{~nm}^{-1}$ and $q_{2} \approx 1.90 \mathrm{~nm}^{-1}$ are observed. With increasing $N / P, q_{1}$ and $q_{2}$ are simultaneously shifted toward lower $q$ values reading their minima of $q_{1}=1.73 \mathrm{~nm}^{-1}$ and $q_{2}=1.88 \mathrm{~nm}^{-1}$, respectively, at $N / P \approx 0.2$. Following, the peak position $q_{1}$ is monotonically moving towards higher $q$-values whereas $q_{2}$ is leveling off at $q_{2}=1.90 \mathrm{~nm}^{-1}$. Eventually, for $N / P>1.8$, this corresponds to $x>2200 \mu \mathrm{m}$ 


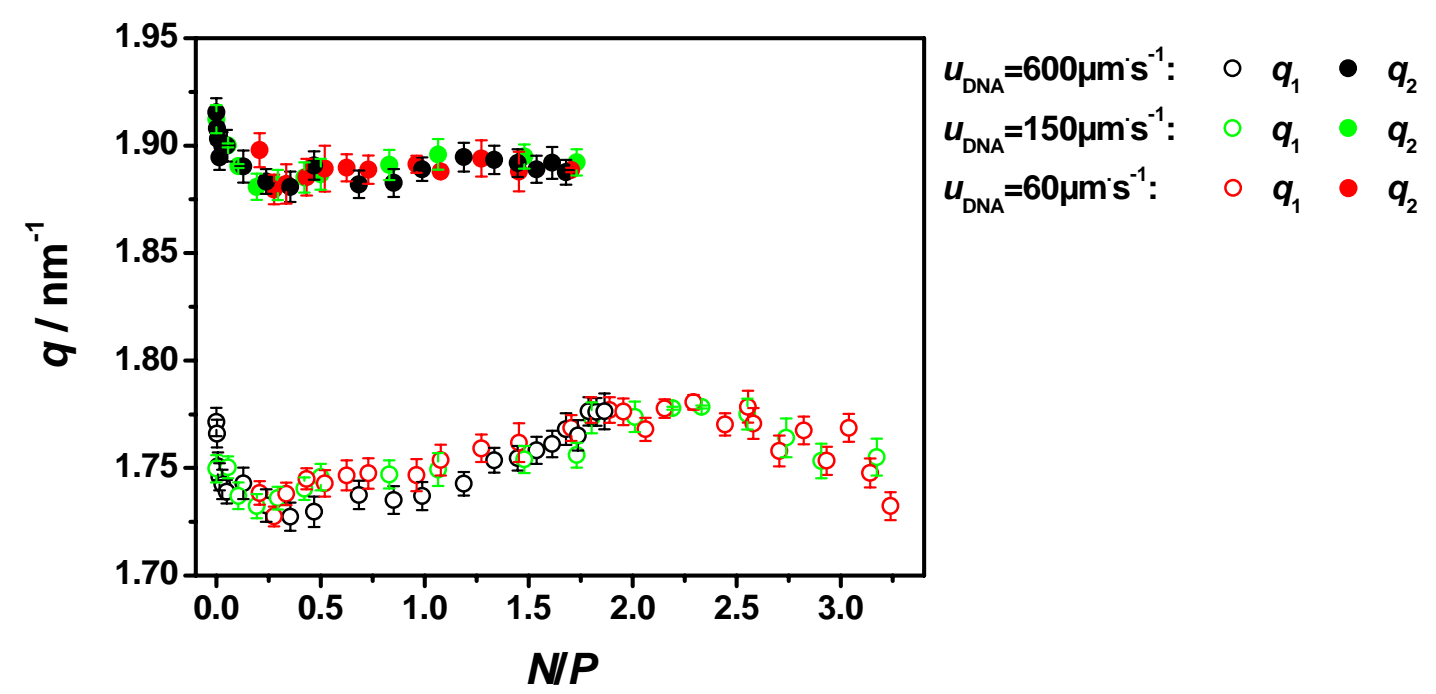

Figure 9-6: Complex composition dependence of peak positions $q_{1}$ and $q_{2}$.

in Figure 9-4, the peak at $q_{2}$ is disappeared leaving a single peak at $q_{1}$. Associated with the disappearance of the peak at $q_{2}$, the remaining peak shows a maximum in $q$-position at $q_{1}=1.78 \mathrm{~nm}^{-1}$. With further increasing $\mathrm{H} 1$ concentration, $q_{1}$ is shifted to smaller $q$ values yielding $q_{1}=1.72 \mathrm{~nm}^{-1}$ at the furthermost observable position $x=13000 \mu \mathrm{m}$ along the reaction channel $(N / P \approx 3.3)$.

Owing to the absence of higher order peaks, the detailed structure of formed H1/DNA mesophases cannot be ruled out. This is mainly due to the fact that spatial constrains of the beamline limited the observable $q$-range to $q<2.67 \mathrm{~nm}^{-1}$. Furthermore, correlation lengths of formed H1/DNA complexes are on the order of 10-70nm (discussed in chapter 9.3.2 and 9.4.2). For systems with such a reduced long-range ordering, scattering profiles often display only strong (100) and (110) reflections. ${ }^{316}$

Structural organization in mesophases with square, hexagonal, or lamellar symmetry is possible. PAMAM dendrimers generation 3 and PPI dendrimer generation 4 exhibit mesophases with hexagonal in-plane symmetry and square in-plane symmetry (chapter 7.2 and references $53,59,61,214$ ). Possessing sizes and charges well comparable to these dendrimers, it is seems reasonable to assume that DNA mesophases formed with H1 have a similar structure as the one observed for dendrimer/DNA, i.e. of hexagonal or/and square symmetry. The (110) Bragg reflection of such a structure is expected at a position $q_{1} \cdot \sqrt{2} \approx 2.43 \mathrm{~nm}^{-1}$, which is well situated in the accessible $q$-range and should be therefore observable. Furthermore, minima of the form factor of the globular domain which could account for the absence of the (110) refection are situated at 1.55 and 


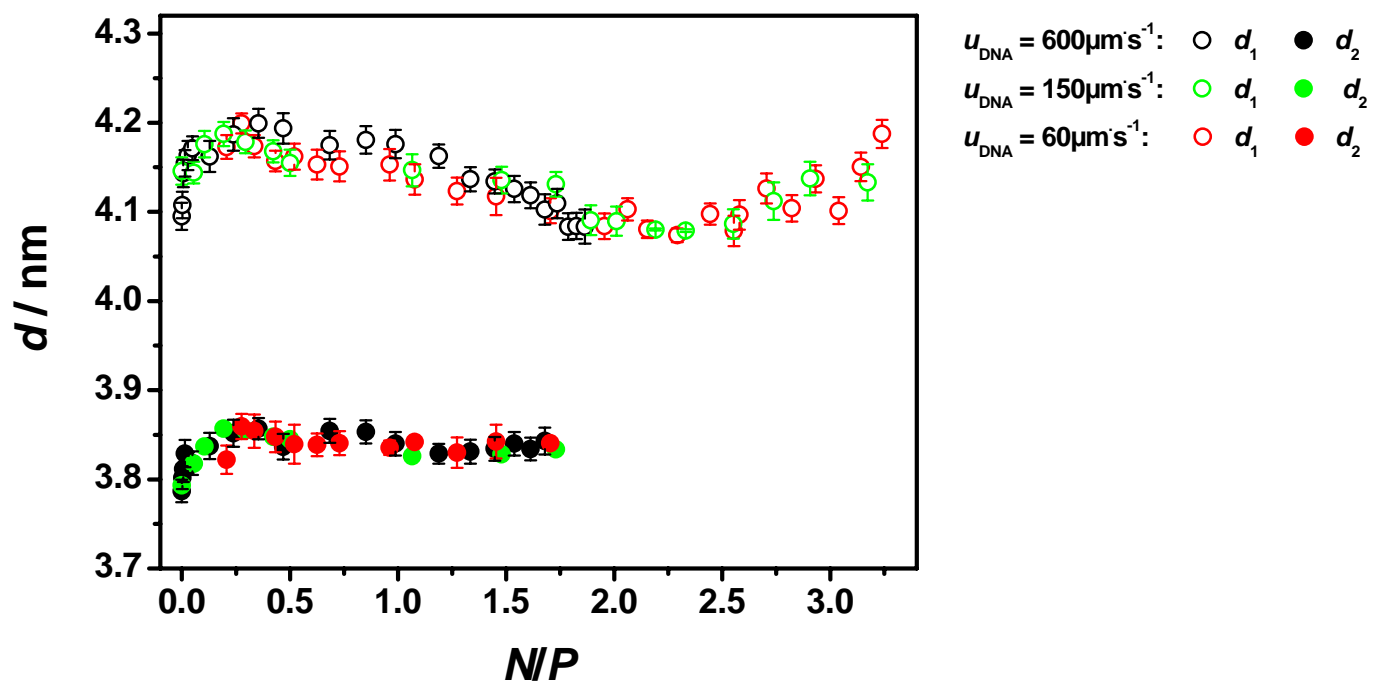

Figure 9-7: Dependence of the lattice spacing $d$ on $N / P$.

$2.66 \mathrm{~nm}^{-1}$ and are therefore not expected to be of influence. Accordingly, from the absence of a peak at this position, the in-plane structure of the mesophase exhibiting the peak at $q_{1}$ can be ruled out most likely to be a hexagonal one. Unfortunately, it is not possible to narrow down the structure of the mesophase exhibiting the peak at $q_{2}$.

Assuming hexagonal ordering, lattice spacing $d$ can be calculated according to following relation: $d=4 \pi / \sqrt{3} q$. In Figure 9-7, lattice spacings $d_{1}$ and $d_{2}$ corresponding to $q_{1}$ and $q_{2}$ are given and their dependence on H1/DNA complex composition - expressed in terms of the charge ratio $N / P$ - shown. Observed lattice spacings are in the range of $3.8-4.2 \mathrm{~nm}$.

The complex interaction of $\mathrm{H} 1$ proteins and DNA resulting from specific $\mathrm{H} 1$ properties is reflected by the unique structure of their assemblies. From electron microscope experiments reported in literature, it is known that the interaction of H1 with DNA at low ionic strength results in the formation of DNA double-fibers linked together by a central core of linker-histone proteins. ${ }^{273}, 307,308,312$ This so called tramtrack configuration of H1/DNA complexes has an effective diameter of the double-fiber of $3.8 \mathrm{~nm},{ }^{306}$ which is in good agreement with observed lattice spacings.

Strikingly, such tramtrack like linker-histone/DNA structures have been found with the globular domain alone. ${ }^{308,312}$ Removal of the positive charge at either of the two DNA binding sites at opposed sides of the globular domain impairs or abolishes the ability to assemble complexes of such structure with DNA. Therefore, the tramtrack like structure of H1/DNA complexes reflects the divalent character of the globular domain. The fact that the most important requirement for binding of $\mathrm{H} 1$ seems to be the 


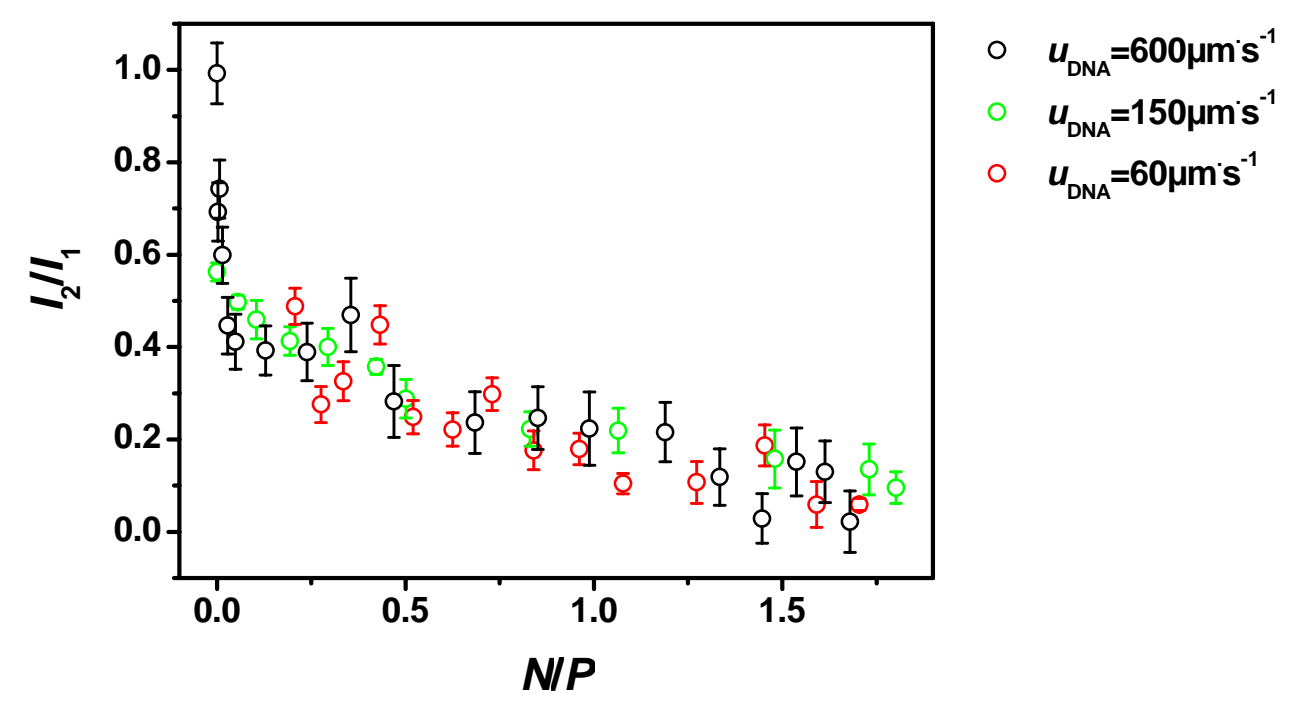

Figure 9-8: Dependence of the intensity ratio $I_{2} / I_{1}$ of the two peaks at $q_{2}$ and $q_{1}$ on the local $N / P$ ratio.

possibility of more than one simultaneous H1/DNA contact may explain not only the tramtrack like complex structure involving two DNA strands braided together by $\mathrm{H} 1$ but also the preference of $\mathrm{H} 1$ for supercoiled over linear DNA. ${ }^{273,307-309,312,317}$ Figure 9-7 shows that both types of H1/DNA mesophase structures have a similar lattice spacing throughout all complex compositions exhibiting changes smaller than $0.25 \mathrm{~nm}$. This readily reflects the fact that it is the globular domain that primarily defines distances between neighbouring DNA strands. ${ }^{308,312}$

In order to enable comparability with results in literature, it is useful to translate $N / P$ into mass fraction $w / w$ of $\mathrm{H} 1$ to DNA. The X-ray data presented in Figure 9-7 exhibit a maximal lattice spacing of both coexisting phases at $N / P \approx 0.2(w / w \approx 0.2)$. This is in good agreement with the fact that for linear DNA molecules and low-salt conditions small amounts of $\mathrm{H} 1, w / w \approx 0.15$, produce complete incorporation of all DNA molecules into extremely large aggregates. ${ }^{309}$

The observed X-ray patterns can be interpreted in terms of a structural transition of H1/DNA complexes from a microdomain structure giving rise to the peak at $q_{2}$ to the other structure existing in the complex yielding the peak at $q_{1}$. A coexistence regime of the two phases is observed for low $N / P$ ratios. In Figure 9-8, the evolution of the structure formation is monitored in terms of the intensity ratio $I_{2} / I_{1}$ of the two Bragg reflections. The coexistence regime occurs over a relatively wide range of $N / P$ ratios. It is characterized by an overlapping of the two relatively sharp Bragg reflections at $q_{1}$ and $q_{2}$. With increasing $N / P, I_{2} / I_{1}$ is gradually reduced reaching zero at $N / P \approx 1.8$. This is the complex composition at which the peak at $q_{2}$ is lost completely. The scattering is 
further on characterized by a single peak at $q_{1}$ that both shifts to lower $q$ values and broadens in $q$ with a further increase of $N / P$.

Combining X-ray microdiffraction and microfluidics allows for time-resolved access to linker-histone/DNA structure formation dynamics. The observed X-ray patterns indicate that the interaction of H1 with DNA is clearly a two step process. First, H1/DNA interaction results in the formation of microdomains yielding the peak at $q_{2}$. After binding, a successive rearrangement of molecules in the formed complexes results in a structure yielding the peak at $q_{1}$. This conformational transition can be monitored in terms of the intensity ratio $I_{2} / I_{1}$ of the two peaks.

The coexistence region is ending at $N / P \approx 1.8$, which corresponds to $15 \mathrm{bp}$ of DNA per each $\mathrm{H} 1$ molecule. This is in good agreement to the excluded binding site determined from sedimentation titration binding data using radioactively labeled linker-histones. ${ }^{318}$ Linker-histones have been found to bind up to a binding density of one protein molecule per $10-13 \mathrm{bp}(N / P \approx 2.5-1.9)$, upon which the DNA lattice is saturated. The excluded binding site has been shown to be relatively independent of salt concentration in the range of $c_{\text {salt }}=14 \mathrm{mM}-350 \mathrm{mM} \cdot{ }^{314,318}$ Results obtained from the presented X-ray microdiffraction experiments, which have been performed at conditions of no additional salt added, agree well to this finding. Moreover, the observation that each $\mathrm{H} 1$ protein binds to $15 \mathrm{bp}$ of DNA agrees remarkably well with results from nuclease digestion of chromatin, which show that each linker-histone protects approximately 10bp from each end of the chromatosomal DNA. ${ }^{9,19,20}$ The exact nature of the two step process of H1/DNA interaction is discussed in chapter 9.3.3.

The experimental setup does not allow to fully distinguish between reaction time and composition dependent effect. In Figure 9-3b, the dependence of $N / P$ on the reaction time $t$ is given for all three flow velocities. For flow velocities of $u=150 \mu \mathrm{ms}^{-1}$ and $600 \mu \mathrm{m} \cdot \mathrm{s}^{-1}, N / P \approx 1.8$ is reached for $t \approx 2.5 \mathrm{~s}$. For $u=60 \mu \mathrm{m} \mathrm{s}^{-1}$ this complex composition is only reached at $t \approx 4.1 \mathrm{~s}$. As mentioned in chapter 9.2 , the deviations are due to different velocity dependent strain rates. However, although the reaction time is almost doubled for $u=60 \mu \mathrm{m}^{-1}$, a vanishing of the peak at $q_{2}$ is only observed for $t \geq 4.1 \mathrm{~s}$. This indicates that the complexation mechanism of H1 and DNA is rather diffusion limited. This is in good agreement with observations of similar sized dendrimer reported in chapter 7 . 


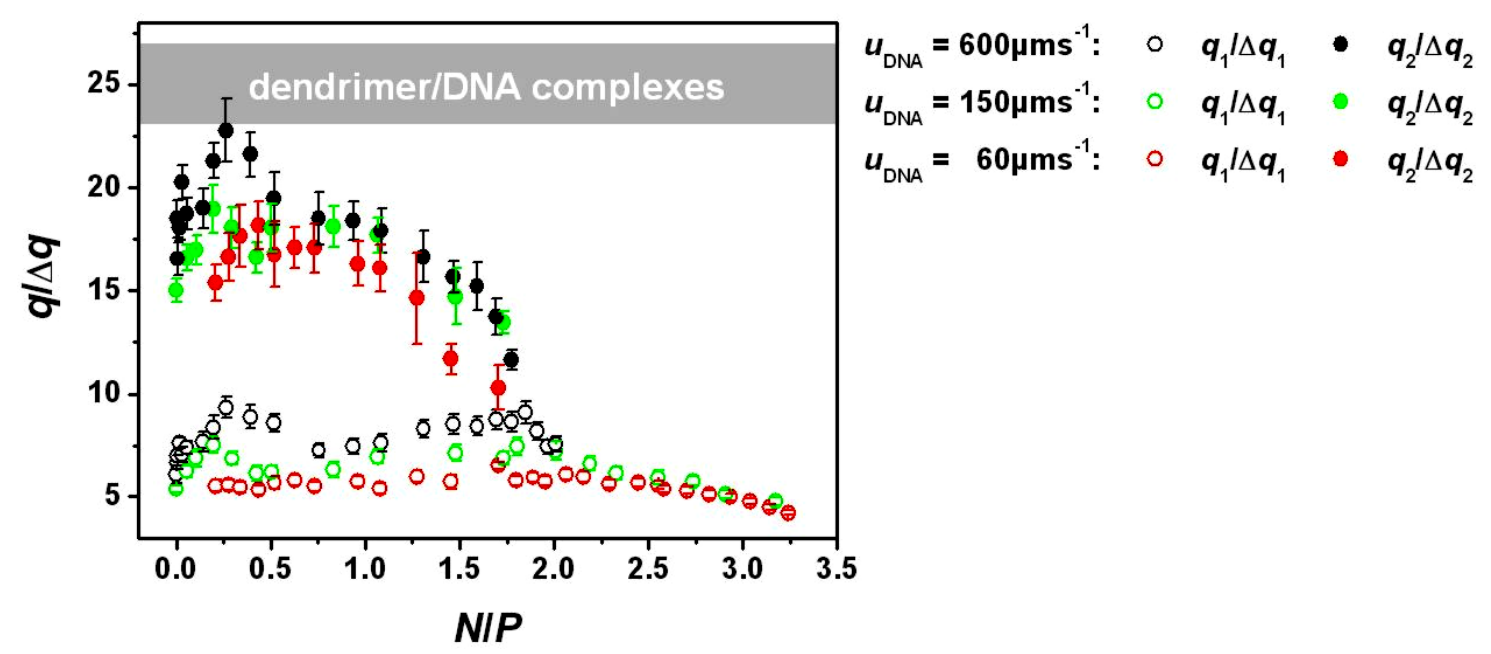

Figure 9-9: $\quad N / P$ dependence of $q_{1} / \Delta q_{1}$ and $q_{2} / \Delta q_{2}$. Corresponding values of dendrimer/DNA complexes are given by the grey box for comparison.

\subsubsection{Microdomain sizes of H1/DNA complexes}

In addition to peak positions, average in-plane microdomain sizes of H1/DNA complexes can be determined from the full width at half maximum $\Delta q$ of the reflections at $q_{1}$ and $q_{2}$ (Figure 9-4). The microdomain size is corresponding to a typical correlation length $L_{\mathrm{C}}=2 \pi / \Delta q$. In order to ensure comparability, it is useful to analyze $q / \Delta q$, which corresponds to the correlation lengths given in terms of the lattice spacing, $q / \Delta q=L_{\mathrm{C}} / d$. The $N / P$-dependence of $q_{1} / \Delta q_{1}$ and $q_{2} / \Delta q_{2}$ are shown in Figure 9-9. A clear dependence on the flow velocity of both $q_{1} / \Delta q_{1}$ and $q_{2} / \Delta q_{2}$ is observable. This will be discussed in detail in chapter 9.4.

At low $N / P$ ratios in the coexistence region, $q_{2} / \Delta q_{2}$ is significantly higher than $q_{1} / \Delta q_{1}$ exhibiting values of $15-23\left(L_{\mathrm{C} 2} \approx 48-73 \mathrm{~nm}\right)$ and $5-9\left(L_{\mathrm{C} 1} \approx 19-33 \mathrm{~nm}\right)$, respectively. With increasing $N / P, q_{2} / \Delta q_{2}$ shows a strong decrease starting around the charge neutral point characterized by $N / P=1$. For all three flow velocities, maximal values $q_{1} / \Delta q_{1}=6$, 7 and 9 , respectively, are found at $N / P \approx 1.8$ when the feature at $q_{2}$ is disappeared. This finding is consistent with the evolution of the ratio of intensity $I_{2} / I_{1}$ shown in Figure 9-8. Parallel to the observed shift in peak position $q_{1}$ to smaller $q$ values with further increasing $\mathrm{H} 1$ concentration, microdomain sizes are decreasing to about $4 \cdot d$ at the latest recorded complex composition $(N / P \approx 3.3)$.

Giving $q / \Delta q$ allows for comparison of H1/DNA microdomain sizes to values obtained from dendrimer/DNA complexes. Compared to linker-histones, PPI generation 4 and PAMAM generation 3 dendrimers have a similar size and charge (chapter 7). This is 
readily reflected in the fact that their DNA complexes have similar lattice spacings $\left(d_{\mathrm{PPI} 4}=3.1-3.6 \mathrm{~nm}, d_{\text {PAMAM3 }}=3.8-4.3 \mathrm{~nm}, d_{\mathrm{H} 1}=3.8-4.2 \mathrm{~nm}\right)$. At comparable strain rates $\left(\dot{\varepsilon}_{\max } \approx 1-2 s^{-1}\right)$ and charge ratios $(N / P \approx 1)$, PPI 4/DNA and PAMAM 3/DNA complexes exhibit microdomain sizes of approximately $23 \cdot d$ and $27 \cdot d$, respectively. These values are comparatively close to those of $q_{2} / \Delta q_{2}$ and differ significant from $q_{1} / \Delta q_{1}$

\subsubsection{Linking $\mathrm{H} 1$ structure to function}

$\mathrm{H} 1$ proteins act as electrostatically driven linker-molecules. At low $N / P$ ratios, a two step reaction process is observable. Both types of H1/DNA mesophases coexist exhibiting significant differences in correlation lengths of $L_{\mathrm{C} 2} \approx 2.5-3 \cdot L_{\mathrm{C} 1}$. Since both types of mesophases experience identical experimental conditions $(N / P, \dot{\varepsilon}, \ldots)$ in the coexistence regime, it is most suggestive that a conformational change of the DNA is occurring during the transition from one H1/DNA mesophase structure to the other.

Compared to other (globular) cationic compaction agents of similar size and charge, e.g. dendrimers, the substantial difference between these and linker-histones is the fact that linker-histones possess highly charged tails. Moreover, although the globular domain is essential for H1/DNA interaction, it is the C-tail that imparts to H1 its DNA binding ability by braiding together the two DNA strands to which the globular domain is bound. Therefore, it seems obvious to assume that the tails are inducing the conformational change of the DNA upon binding. Independent of the assumed model of underlying interaction mechanisms, many studies reported in literature indicate that such a conformational transition of the DNA is indeed involved in H1/DNA interaction. $^{314,318-320}$

Both linker-histone tails, which are unfold in solution, exhibit structure when exposed to helix-stabilizer (e.g. trifluoroethanol, $\mathrm{HClO}_{4}$ ) and attain optimum secondary and hence tertiary structure upon interaction with DNA. ${ }^{280-286}$ Experimentally, structures of a turn and of a helix-turn motif have been determined by NMR. ${ }^{282}$ Therefore, the presented experimental X-ray data suggest that the conformational transition of $\mathrm{H} 1$ tails - in particular of the C-tails ${ }^{280-286}$ - from their rather extended conformation in aqueous solution to their fully folded state upon interaction with DNA is the motor for the observed conformational phase transition of H1/DNA complexes. In the first step of the non-equilibrium structure formation, $\mathrm{H} 1$ proteins bind to DNA unspecifically maintaining their rather extended tail conformations from solution. This leads to the 


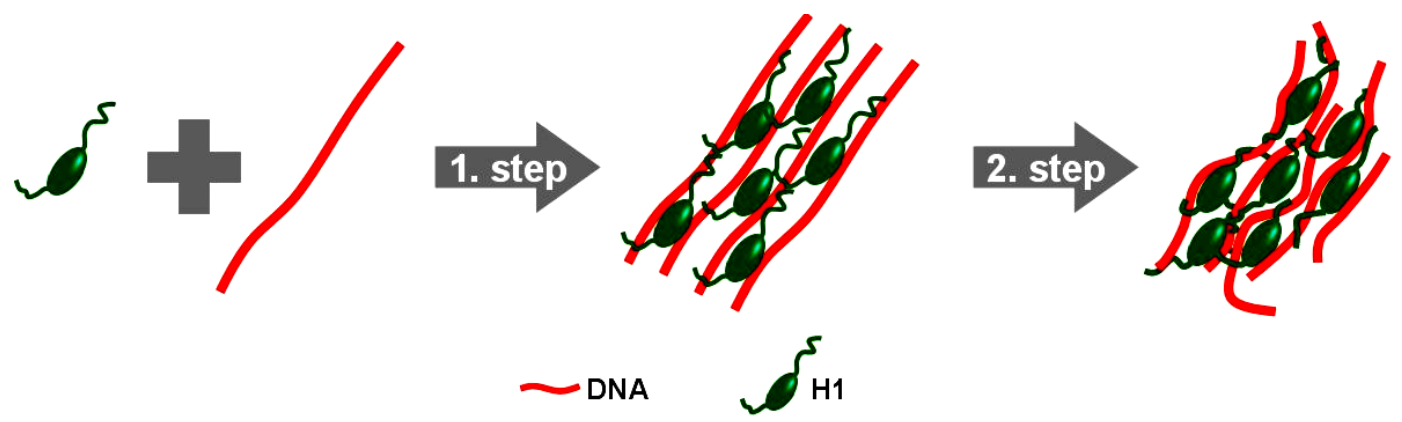

Figure 9-10: Schematic representation of the H1/DNA interaction. In a first step, H1 molecules bind unspecificly to DNA with extended tails. In a second step, $\mathrm{H} 1$ tails fold upon interaction with DNA, distorting and bending thereby the DNA structure.

formation of complexes with microdomain sizes well comparable to those known from dendrimer/DNA complexes. However, interacting with DNA alleviates for the associated entropic penalty loss and consistently a transition of the extended tails to their folded structure occurs. This two step binding mechanism of H1 to DNA is schematically shown in Figure 9-10. The coexistence of the two mesophase structures can therefore be explained by assuming two types of microdomains, one including linker-histones with still rather extended tails, the other comprising already completely folded tails.

The HMG-box protein fold (HMG: high-mobility group) has been proposed to be the most appropriate candidate template for modeling the C-tail. ${ }^{321}$ According to this structural feature, linker-histone tails are speculated to completely wrap around the DNA distorting thereby the helical structure. ${ }^{283,}{ }^{322-324}$ Such a DNA bending as well as an unwinding of supercoiled DNA has been observed upon the interaction with linkerhistones. $^{283,325}$ Consistently, the two different conformational forms of DNA correspond to undistorted DNA and distorted, bended DNA due to completely encompassing of the DNA chain by folded C-tails. Moreover, one can speculate that the significantly larger microdomain size of H1/DNA complexes yielding the peak at $q_{2}$ is directly reflecting the rather undistorted and unbended nature of incorporated DNA chains. Complete saturation of all DNA binding sites with $\mathrm{H} 1$ corresponds to a complex conformation comprising only fully folded tails, which is observed at $N / P \approx 1.8$ (15bp per $\mathrm{H} 1$ ).

This appears reasonable since folded tails leave more unoccupied space along the DNA chains. After the charge neutral point characterized by $N / P=1$, a further increase of $N / P$ leads to a positive overcharging of H1/DNA complexes. Figure 9-9 shows that after $N / P \approx 1.1$ ( $28 \mathrm{bp}$ of DNA per each H1 molecule; $w / w \approx 1.2$ ), the correlation length 


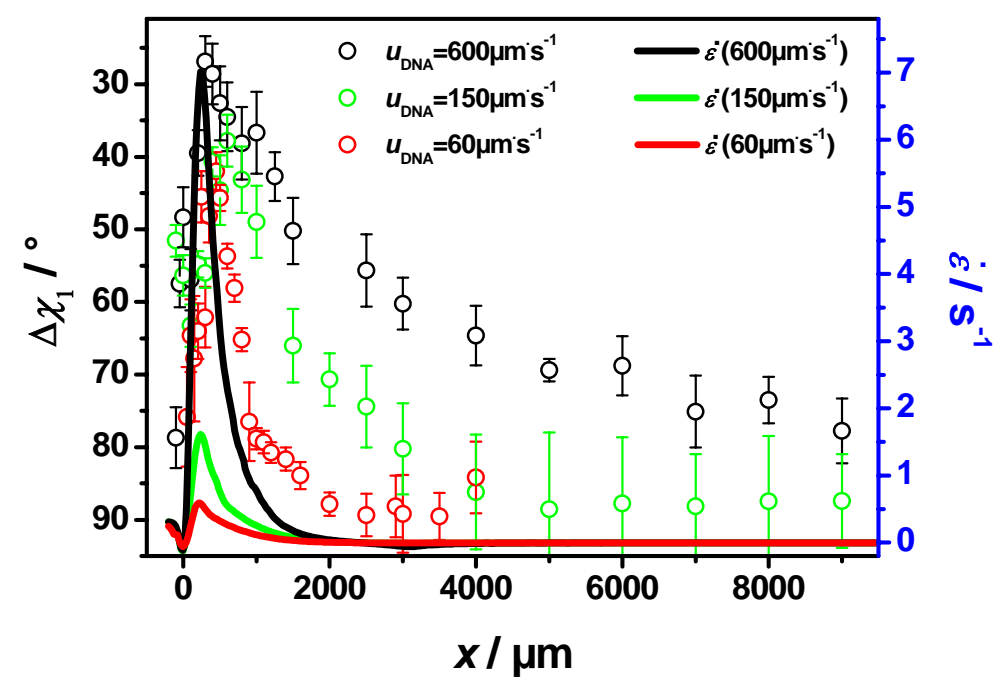

Figure 9-11: Degree of orientational order $\Delta \chi_{1}$ in dependence of the $x$-position. Solid lines represent the strain rate $\dot{\varepsilon}$ inducing the preferential orientation. Note that the ordinate representing $\Delta \chi_{1}$ is inverted.

of microdomains with extended tails is rapidly reduced. This may indicate that the increasing electrostatic repulsion between neighboring $\mathrm{H} 1$ molecules affects the folding of C-tails. Compared to binding of linker-histones to nucleosomal core particles in vivo, it may be likely that tails of core histones are interacting with $\mathrm{H} 1$ tails and induce thereby the transition to a folded state.

\subsection{Demonstration of certain fundamental principles of microfluidics encountered for complex fluids}

In chapter 9.3, the combination of small angle X-ray microdiffraction and microfluidics has been used to analyze the interaction of linker-histones with DNA and to gain new insights in linker-histone/DNA structure formation. In turn, the H1/DNA system can be used to demonstrate certain basic principles of microfluidics, which one encounters when using complex fluids. Moreover, it is used to demonstrate in detail the significant advantages for the characterization of complex fluids provided by combining X-ray microdiffraction and microfluidics. 

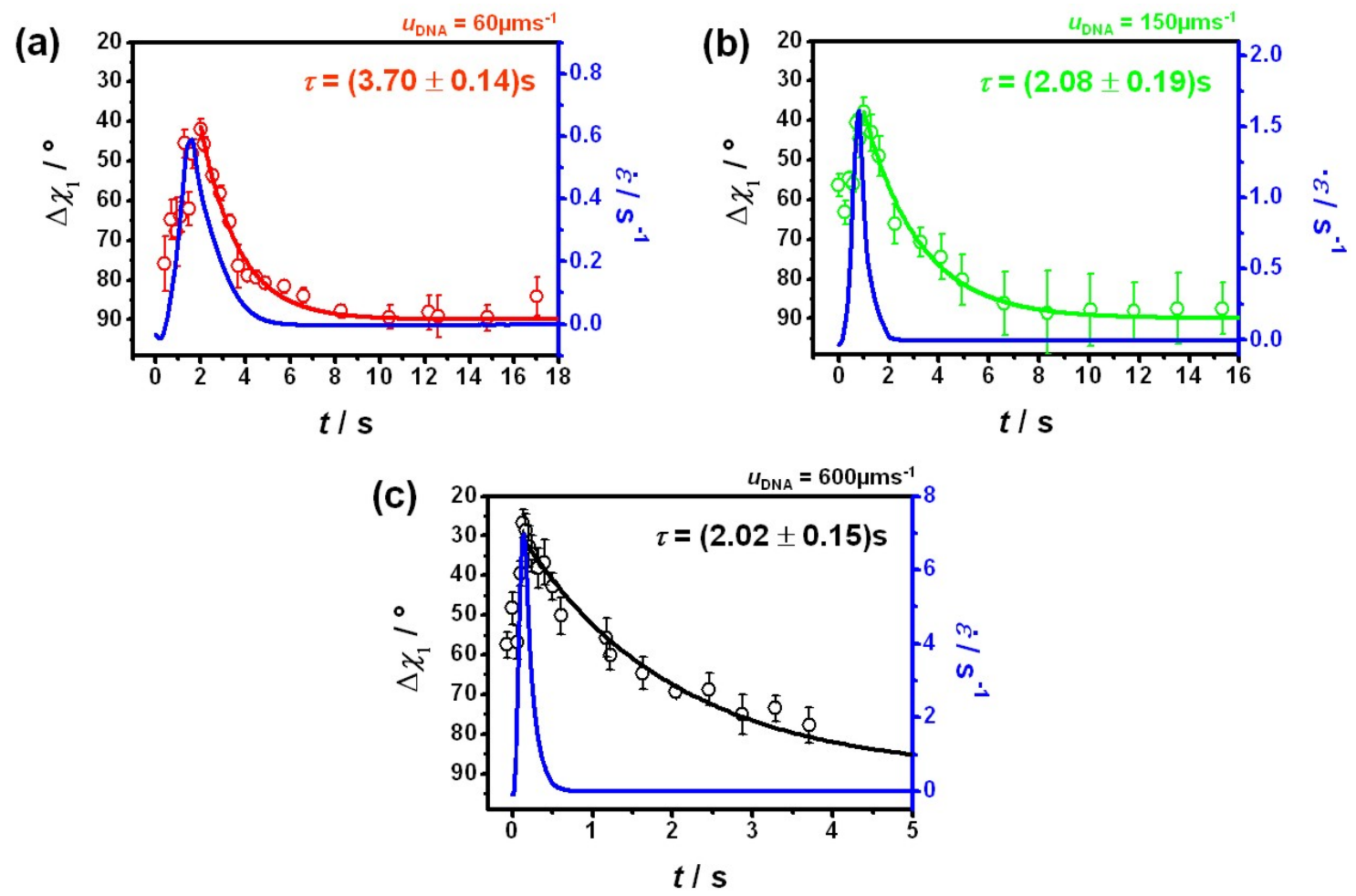

Figure 9-12: Time dependence of the degree of orientational order $\Delta \chi_{1}$ and strain rate $\dot{\varepsilon}$ (blue lines). Solid lines represent fits to data using first order exponential decay functions. Note that the ordinate representing $\Delta \chi_{1}$ is inverted.

\subsubsection{Flow induced orientation of biomaterials}

Owing to the acceleration of fluid elements at the confluence of main and side channels, DNA molecules as well as H1/DNA assemblies experience hydrodynamic strain, which induces an orientation of formed microdomains. In Figure 9-4, representative $2 \mathrm{D} \mathrm{X}$-ray diffraction patterns are shown. At positions $x$ close to the confluence area, pattern clearly reveal oriented diffraction rings. However, moving down the reaction channel, a loss in orientational order is readily seen. The hydrodynamic focusing setup enables one to follow the entire orientational dynamics of microdomains from the inception of flow-induced orientation to the fully orientationally relaxed biomaterials. By changing flow velocities and/or observation position, it is possible to characterize changes in the orientational distribution of microdomains with hydrodynamic strain and to simultaneously vary complex composition. 


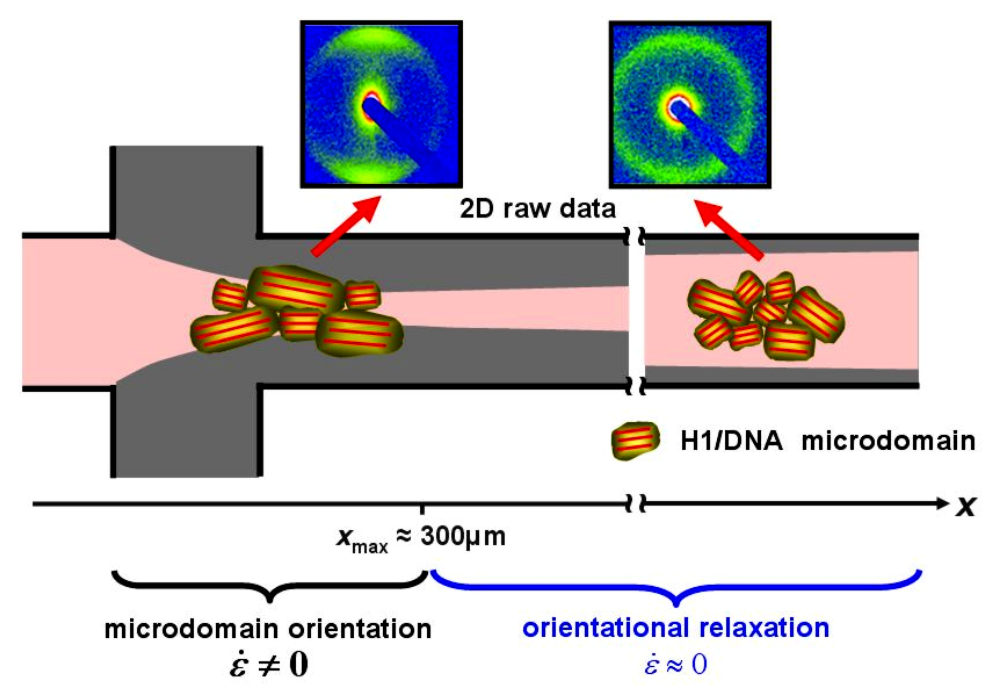

Figure 9-13: Schematic representation of microdomain orientation and successive orientational relaxation along the outlet channel.

Scans of the intensity along the azimuthal angle $\chi$ on the rings of the peaks at $q_{1}$ and $q_{2}$, respectively, both reveal a strong preferential microdomain orientation along the flow direction $\left(\chi \approx \pm 90^{\circ}\right)$, i.e. DNA molecules align parallel to the applied strain. The degree of orientation can be quantified as the full width at half maximum $\Delta \chi_{1}$ and $\Delta \chi_{2}$ of azimuthal peaks $q_{1}$ and $q_{2}$, respectively. Figure 9-11 shows the dependence of $\Delta \chi_{1}$ on the position $x$ along the reaction channel for the three different flow velocities. Since $\Delta \chi_{2}$ exhibits a qualitatively identical behavior, data are not shown. The degree of orientational preference $\Delta \chi_{1}$ for each data set is contrasted to the effective strain rate (solid lines of corresponding color), which describes mechanical effects on the biomaterials under flow. The hydrodynamic strain rate $\dot{\varepsilon}=\partial v / \partial x$ is calculated from simulated velocity fields given in Figure 9-2 exemplarily for $u_{\mathrm{DNA}}=600 \mu \mathrm{m} \mathrm{s}^{-1}$.

Beginning at the confluence of main and side streams, local strain rates increase. Remarkably, the position of maximum strain rate is situated independent of applied flow velocities at $x_{\max } \approx 300 \mu \mathrm{m}$ (Figure 9-11). Maximal strain rate values are $\dot{\varepsilon}=0.6 \mathrm{~s}^{-1}$, $1.6 \mathrm{~s}^{-1}$, and $7.0 \mathrm{~s}^{-1}$ for the three different initial flow velocities of $u_{\mathrm{DNA}}=60,150$ and $600 \mu \mathrm{m}^{-1}{ }^{-1}$, respectively.

In order to elucidate the dynamics of the orientation process, in Figure 9-12 for each flow velocity the time dependence of $\Delta \chi_{1}$ is shown. Corresponding strain rates are given for comparison. Owing to different flow velocities, for each data set $x_{\max }$ translates into a different reaction time featuring maximal $\dot{\varepsilon}$ and - associated with this - maximal degree of orientation $\left(t_{60}=1.6 \mathrm{~s}, t_{150}=0.8 \mathrm{~s}\right.$, and $\left.t_{600}=0.2 \mathrm{~s}\right)$. After having reached its maximal value, the strain rate decays exponentially to zero. The length of 
the time interval $\Delta t$ in which an effective strain rate is active depends on the flow velocity. The higher the flow velocity is the narrower $\Delta t$ and so much the better the effective strain can be regarded as momentary perturbation acting on the biomaterials in flow. This can be readily seen in Figure 9-12. The magnitude of the orientation inducing hydrodynamic strain rate is highly depending on initial flow velocities as therefore is $\Delta \chi_{1}$. The initial fast rise in the preference of microdomain orientation occurs simultaneously with $\dot{\varepsilon}$. Maximal preference of orientation corresponds to minimal values of $\Delta \chi_{1} \approx 27^{\circ}, 38^{\circ}$ and $42^{\circ}$, found for initial flow velocities of $u_{\text {DNA }}=600,150$ and $60 \mu \mathrm{ms}^{-1}$, respectively. After the orienting influence is removed, the system relaxes. Figure 9-13 gives a schematic representation of the process of microdomain orientation and the successive orientational relaxation.

The orientational relaxation dynamics of microdomains can be described by a first order exponential decay function with a single characteristic relaxation time $\tau$ (solid lines in Figure 9-12), which is derived from each data set: $\tau_{60}=(3.70 \pm 0.14) \mathrm{s}$; $\tau_{150}=(2.08 \pm 0.19) \mathrm{s} ; \quad \tau_{600}=(2.02 \pm 0.15) \mathrm{s}$. Orientational relaxation times for initial flow velocities of $u_{\mathrm{DNA}}=150$ and $600 \mu \mathrm{ms}^{-1}$ are in excellent agreement, only the relaxation time determined for $u_{\mathrm{DNA}}=60 \mu \mathrm{m}^{-1}$ shows deviations from $\tau \approx 2 \mathrm{~s}$. The observed deviation in relaxation time for this slowest flow velocity can be explained by the fact that the time interval $\Delta t$, in which the microdomain orientation inducing hydrodynamic strain is active, is strongly increasing with reducing velocity. Therefore, the hydrodynamic strain is not behaving as a momentary perturbation of the system any more and $\Delta \chi_{1}$ is rather following the decay of $\dot{\varepsilon}$ than exhibiting an unperturbated relaxation process (Figure 9-12c). From the fact that obtained relaxation times for initial flow velocities of $150 \mu \mathrm{ms}^{-1}$ and $600 \mu \mathrm{ms}^{-1}$ are in excellent agreement, it can be concluded that the decay of $\dot{\varepsilon}$ is not playing a decisive role in the relaxation process any more.

\subsubsection{Strain induced variations in microdomain size}

In addition to peak positions, average in-plane microdomain sizes for H1/DNA complexes can be determined by investigating the full width at half maximum $\Delta q$ of the reflections at $q_{1}$ and $q_{2}$ (Figure 9-4). As discussed in chapter 9.3.2, it is usefull to analyze correlation lengths $L_{\mathrm{C}}$ given in terms of the lattice spacings $d: L_{\mathrm{C}} / d=q_{1} / \Delta q_{1}$. Figure 9-14 shows $q_{1} / \Delta q_{1}$ in dependence of the $N / P$ ratio. Since $q_{2} / \Delta q_{2}$ exhibits a qualitatively identical behavior, data are not shown. Plotting $q_{1} / \Delta q_{1}$ against $N / P$ is 


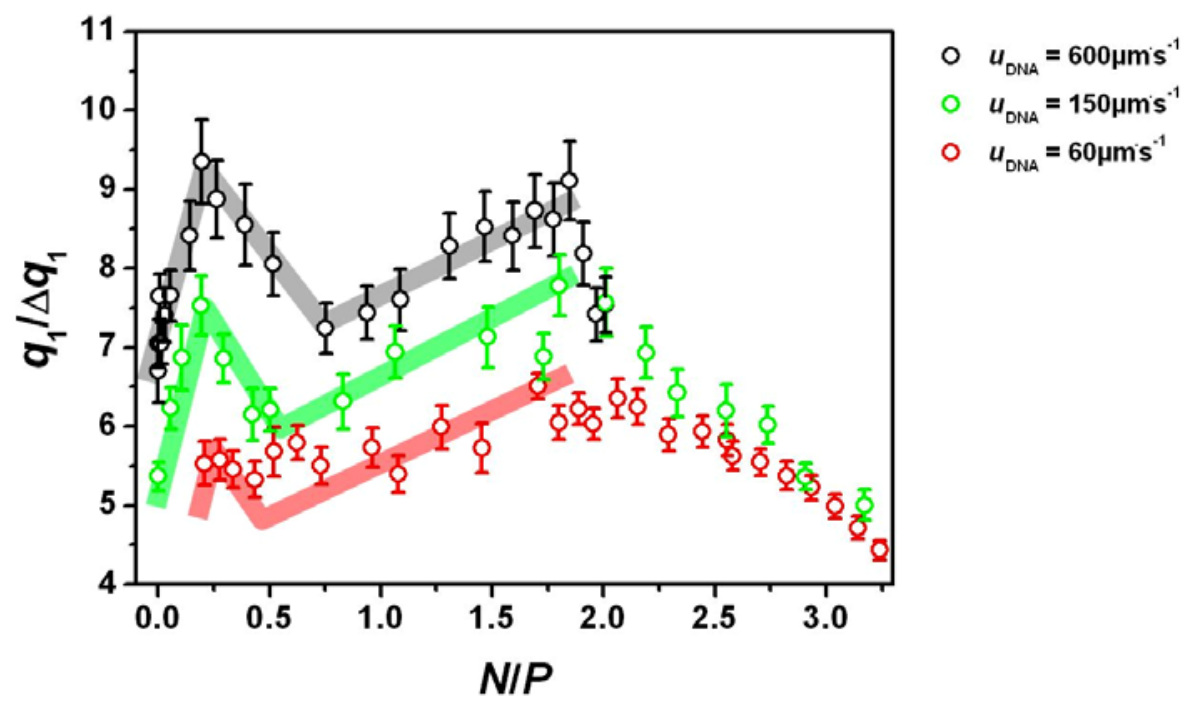

Figure 9-14: Dependence of $\Delta q_{1}$ on complex composition $N / P$. Solid lines are guides to the eyes only.

expected to allow for collapsing of data collected at different flow velocities onto a master plot reflecting the H1/DNA complex composition dependence of $q_{1} / \Delta q_{1}$. In Figure 9-14, however, such a behavior is only partly observable: Although all three data sets are following similar courses and are exhibiting analog systematic changes, their absolute values are significantly different and obviously depending on the magnitude of the flow velocity.

At $N / P \approx 0.2, q_{1} / \Delta q_{1}$ reaches maximal values for all flow velocities. This is exactly the complex composition at which minimal values of $q_{1}$ and $q_{2}$ are found (Figure 9-6). Following, for all three flow velocities maximal values of $q_{1} / \Delta q_{1}=6.4,7.8$ and 9.1, respectively, are found at $N / P \approx 1.8$ when the feature at $q_{2}$ is disappeared. This finding is consistent with the evolution of the ratio of intensity $I_{2} / I_{1}$ shown in Figure 9-8. Parallel to the observed shift in peak position $q_{1}$ to smaller $q$ values with further increasing $\mathrm{H} 1$ concentration, $q_{1} / \Delta q_{1}$ is increasing to its values $q_{1} / \Delta q_{1}=4.4$ at the final complex composition $(N / P \approx 3.3)$.

Besides their dependence on complex composition, Figure 9-14 shows a clear and systematic dependence of $q_{1} / \Delta q_{1}$ on the flow velocity. Throughout all $N / P$ ratios, $q_{1} / \Delta q_{1}$ is increasing with increasing strain rate. This is indicative for a strong correlation of microdomain size and strain induced orientation, which is superimposed to the $N / P$ dependence of $q_{1} / \Delta q_{1}$. In order to discuss this phenomenon, in Figure 9-15 $q_{1} / \Delta q_{1}$ is given in dependence of the position $x$ along the reaction channel since local strain rates are intrinsically depending on $x$ and not on $N / P$ (chapter 9.4.1). The abscissa is divided 


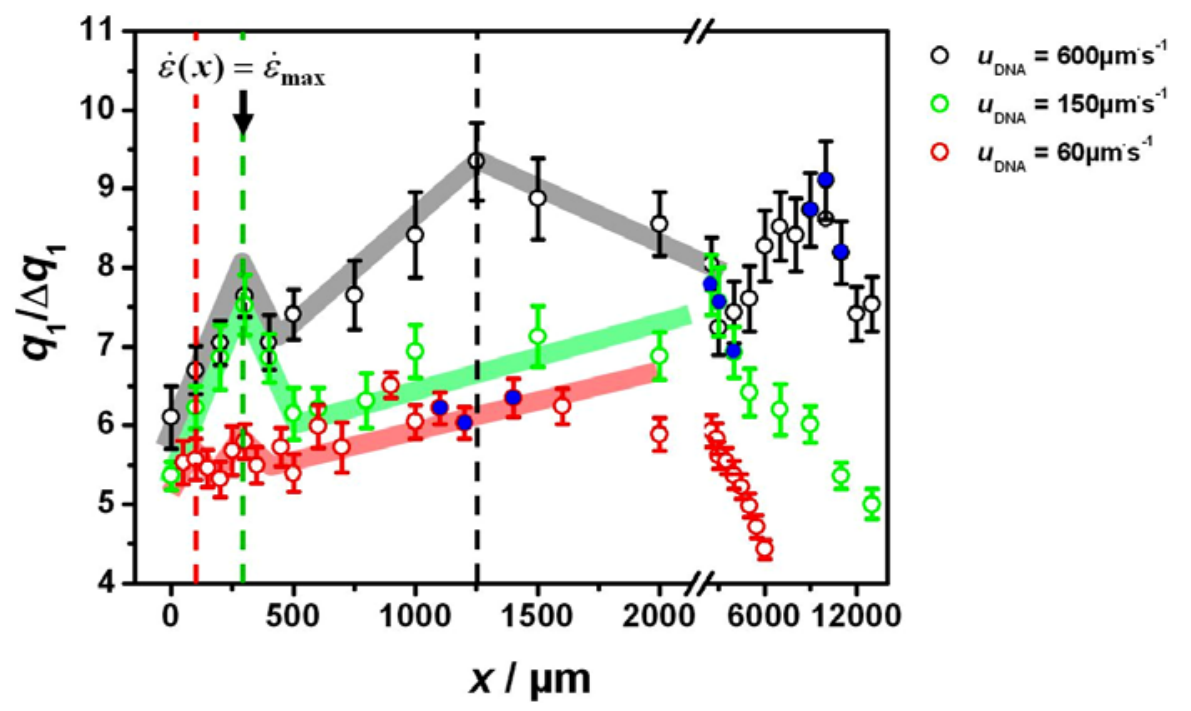

Figure 9-15: Dependence of $\Delta q_{1}$ on the position $x$ along the reaction channel. The black arrow indicates the position of maximum strain rate $\dot{\varepsilon}_{\max }$. The dashed lines mark positions corresponding to $N / P \approx 0.2$. Blue points are used to $L_{\mathrm{C} 1}$ in Figure 7-16.

in a way that access to variations in $q_{1} / \Delta q_{1}$ at positions $x<2000 \mu \mathrm{m}$ is improved. As expected, data sets are not showing parallel changes any more. At $N / P \approx 0.2, q_{1} / \Delta q_{1}$ exhibits maxima due to its complex composition dependence as can be readily seen in Figure 9-14. Consistently, $q_{1} / \Delta q_{1}$ adopts maximal values at corresponding $x$-positions marked with dashed lines in Figure 9-15. However, $q_{1} / \Delta q_{1}$ obtained from all three data sets develops an additional maximum at the position of maximum strain rate, $x_{\max } \approx 300 \mu \mathrm{m}$, which is indicated by the arrow in Figure 9-15. For the data set recorded at $u_{\mathrm{DNA}}=150 \mu \mathrm{m}^{-1}{ }^{-1}$, both maxima due to the $N / P$ dependence of H1/DNA complexes and due to hydrodynamic strain are coinciding at $x_{\max } \approx 300 \mu \mathrm{m}$ (green dashed line and arrow in Figure 9-15). The superposition of both effects is responsible for the fact that the data set recorded at $u_{\mathrm{DNA}}=150 \mu \mathrm{m}^{-1}$ exhibits similar $q_{1} / \Delta q_{1}$ values as the data set recorded at the higher flow velocity $u_{\mathrm{DNA}}=600 \mu \mathrm{ms}^{-1}$. The last maximum observed a different position for each curve reflects the position where the feature at $q_{2}$ is disappeared.

These findings provide interesting insights into the response of a 2D mesophase of long chain DNA molecules to external strain. The strain rate induced extensive alignment of H1/DNA assemblies' leads to an increase in $q_{1} / \Delta q_{1}$ with applied external strain. In order to quantify for the strain effect on $q_{1} / \Delta q_{1}$, only data points that accomplish the following conditions must be considered: Firstly, in order to account for compositional 


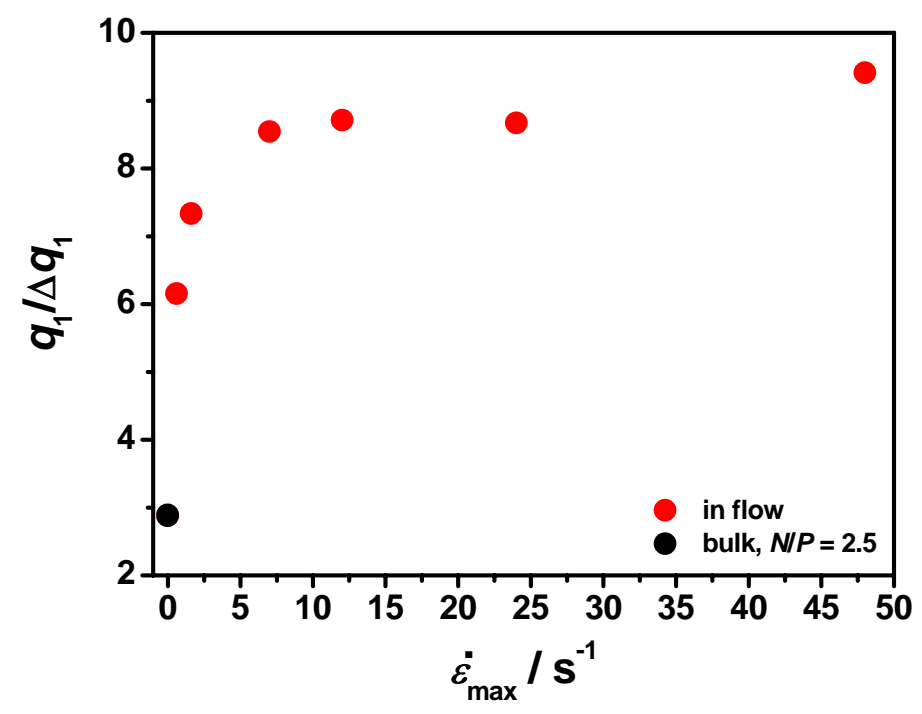

Figure 9-16: Dependence of the microdomain size on the maximal strain rate superimposed. Data from bulk measurements are given for comparison.

changes in $q_{1} / \Delta q_{1}$, considered data points must be recorded from H1/DNA complexes exhibiting similar N/P ratios for all three data sets. Secondly, no hydrodynamic strain must be active any more and the complexes have to be already sufficiently relaxed, i.e. $\Delta \chi_{1} \approx 90^{\circ}$. Only data points marked blue in Figure 9-15 fulfill both conditions simultaneously exhibiting $N / P=1.9-2.0$ and $\Delta \chi_{1}=78^{\circ}-80^{\circ}$. In Figure 9-16, $q_{1} / \Delta q_{1}$ are given in dependence of the maximum strain rate. Data points at $\dot{\varepsilon}_{\max }>10 \mathrm{~s}^{-1}$ are obtained from data sets recorded at initial flow velocities of 1000, 2000, and $4000 \mu \mathrm{ms}^{-1}$, which are not as comprehensive as the three data sets discussed to this point. In addition, $q / \Delta q$ recorded from a bulk sample with a composition of $N / P=2.5$ is shown. Figure 9-16 shows that already a low maximum strain rate of $0.6 \mathrm{~s}^{-1}$ at $u=60 \mu \mathrm{m}^{-1}{ }^{-1}$ results in a dramatic effect the on microdomain size: $q_{1} / \Delta q_{1}$ more than doubles. With increasing strain rate, $q_{1} / \Delta q_{1}$ is continuously increasing up to $\dot{\varepsilon}_{\max } \approx 10 s^{-1}$. Further increasing the strain rates has only minor effects. The fact that it is possible to significantly improve the correlation length of biomaterials by superimposing external strain is a clear advantage of using flow to assemble such materials. In the context of X-ray diffraction analysis, this phenomenon can be utilized to improved the characterization of biomolecular materials, which are difficult to crystallize and normally form liquid-crystalline structures ${ }^{57,63,64}$. 

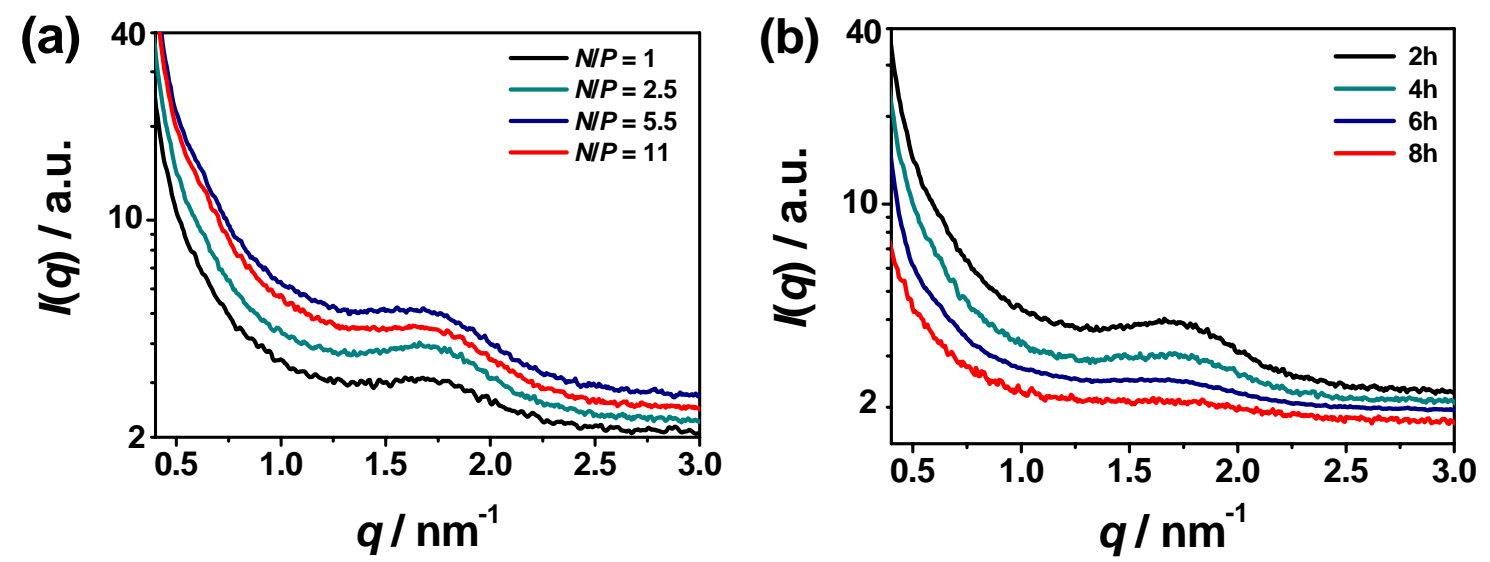

Figure 9-17: (a) SAXS profiles of H1/DNA bulk complexes at different $N / P$. (b) SAXS profiles of successive measurements at a fixed position $(N / P=2.5)$.

\subsubsection{Bulk measurements of H1/DNA complexes}

As a contrast to the X-ray microdiffraction measurements in flow, additional X-ray measurements on H1/DNA bulk samples have been performed in quartz glass capillaries using the in-house setup. Figure 9-17 shows representative small angle Xray scattering data (chapter 2.2).

In Figure 9-17a, X-ray diffraction patterns of complexes with $N / P$ ratios ranging from $N / P=1-11$ are given. For all data sets, exposure times of two hours have been used. Time-resolved X-ray diffraction measurements of H1/DNA structure formation showed that H1/DNA interaction is a two step process. This was reflected in the X-ray pattern by a transition from two peaks at $q_{2}$ and $q_{1}$ to a single peak at $q_{1}$. Despite significant variations in complex composition, X-ray diffraction patterns of the bulk samples are almost unchanged exhibiting a single, broad peak at $q_{1} \approx 1.76 \mathrm{~nm}^{-1}$. This demonstrates a principle limitation of bulk sample analysis. In order to gain new insights in H1/DNA interaction, time-resolved access to a broad range of reaction states seems indispensable.

In Figure 9-17b, SAXS profiles of successive measurements at a fixed position are given $(N / P=2.5)$. With each repeat, the observed peak is gradually reduced in intensity. After a total exposure time of six hours, the peak is almost vanished. Therefore, SAXS profiles in Figure 9-17b clearly demonstrate that H1/DNA samples experience a significant degradation due to the X-ray irradiation during measurements. Although the flux of the synchrotron X-ray beam is about $10^{5}$ times higher compared to that of the in-house setup, the very short times of less than $0.1 \mathrm{~s}\left(u_{\mathrm{DNA}}=60 \mu \mathrm{m}^{-1} \mathrm{~s}^{-1}\right)$, in which the biomaterials are exposed to X-ray irradiation in flow, avoid sample damage 
efficiently. Accordingly, the radiation level at which the biomolecular samples exhibit significant degradation (red curve in Figure 7-17b) are about 3-36 times higher (for $u_{\mathrm{DNA}}=60-600 \mu \mathrm{m} \cdot \mathrm{s}^{-1}$ ) compared to those H1/DNA complexes experience in microfluidic experiments. Therefore, performing X-ray diffraction measurements in flow allows overcoming this fundamental restriction.

Despite extensive study, it is surprising that so far there are no studies published in literature probing linker-histone/DNA interaction dynamics and structure formation by small angle X-ray diffraction. I believe that the fundamental restrictions associated with $\mathrm{X}$-ray measurements of bulk samples demonstrated above are one of the main reasons for this.

\subsection{Conclusions}

Combining X-ray microdiffraction and microfluidics, for the first time, small angle Xray scattering measurements of $\mathrm{H} 1 / \mathrm{DNA}$ complexes have been reported. The experimental setup allowed accessing H1/DNA structure formation dynamics. Observed X-ray patterns indicate that the interaction of H1 with DNA is a two step process: an initial unspecific binding of $\mathrm{H} 1$ proteins to DNA is followed by a rearrangement of molecules in the formed complexes. Consistent with a multitude of studies in literature, obtained X-ray diffraction data indicate that a conformational transition of the DNA upon binding of H1 is involved in H1/DNA interactions. The results presented in this chapter suggest that the conformational transition of the linkerhistone tails from their rather extended conformation in aqueous solution to their fully folded state upon interaction with DNA are most likely responsible for the conformational phase transition of H1/DNA complexes. This kind of rearrangement is most likely necessary to determine the curvature and the path of initial parts of linkerDNA at the entry/exit point of the nucleosome. I believe this finding to have a direct bearing on the understanding of chromatin fiber folding into higher order structures.

Moreover, the H1/DNA system has been used to demonstrate the effect of hydrodynamic strain on complex fluids and to directly follow the orientation and the orientational relaxation of microdomains. The fact that it is possible to improve the correlation length of biomaterials by superimposing strain is a clear advantage of using flow to assemble such materials. In the context of X-ray diffraction analysis, this phenomenon can be utilized to improve the characterization of biomolecular materials, which are difficult to crystallize and normally form liquid-crystalline structures. 


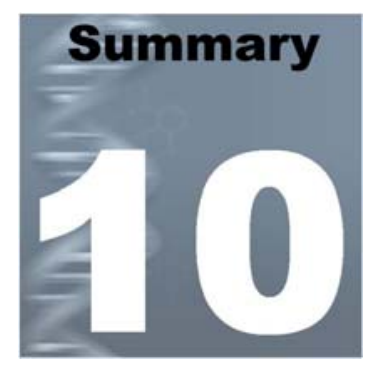

\section{SUMMARY}

In summary, the potent combination of microfluidics with small-angle X-ray microdiffraction and confocal Raman microscopy allows for detailed insights into the evolution of dendrimer and linker-histone induced DNA compaction. In particular, the laminar flow conditions inside microchannels provide the possibility to investigate interaction processes in a time-resolved manner. Diffusive mixing in microchannels creates tunable reaction conditions with defined changes in local concentrations. Multiple experimental data are therefore aquired at any desired complex composition on a single device, while consuming extremely small amounts of material and without any concerns for radiation damage. The fact that it is possible to improve the correlation length of biomaterials, by superimposing stress, is a clear advantage of using flow to assemble such materials, which normally form liquid-crystalline phases. The major obstacle for performing X-ray diffraction measurements directly on a microfluidic chip was the lack of cheap, tunable, and robust devices with thin, lowabsorbing and low-scattering windows. Therefore, the successful development of such $\mathrm{X}$-ray compatible microfluidic devices is a central aspect of the present work. The newly developed microflow foils now place researchers in an advantageous position to approach important biophysical questions regarding biomatter self-assembly and interactions in a variety of environments. It is anticipated that microflow foils will provide a robust and affordable approach not only to continuous flow X-ray microdiffraction, but also in micro total analysis systems (" $\mu$-TAS") and bioassays. Here, two different classes of compaction agents have been studied: dendrimers of varying generation and linker-histones $\mathrm{H} 1$. 
Dendrimers, which are a unique class of precisely engineered, highly branched macromolecules, can be viewed as compact, spherical objects with a smeared surface region. In order to precisely control and understand dendrimer induced DNA compaction, it is of crucial importance to first characterize the compaction agent. Therefore, dendrimer properties have been analyzed in detail. In particular, the phenomenon of charge-induced dendrimer swelling has been experimentally quantified for PPI and PAMAM dendrimers and over a wide range of generations. The results clearly show highly predictable, charge-induced changes in dendrimer conformation. Therefore, the discrepancy between theory and experiments that existed in literature up to now is eliminated. Moreover, the observed response of dendrimers to superimposed electrostatics can be generalized to all types of dendrimers.

Through the variation of dendrimer size and charge, the entire spectrum of naturally occurring condensation agents is bridged, ranging from small cations, such as spermine/spermidine encountered in viruses, to the much larger histone proteins, in eukaryotic cells. Therefore, dendrimers are perfectly suited to study DNA compaction and to mimic the influence of electrostatic interactions on DNA compaction in vivo. In particular, the dynamic assembly of DNA condensates by cationic dendrimers with sizes and charges situated between that of small multivalent organic cations and larger histone-like proteins is analyzed. Performing measurements on the $2 \mathrm{D}$ columnar mesophase formation in flow for the first time enables access to very low $N / P$ ratios in a controlled manner. Most surprising, our results indicate that in an undercharged regime it is possible that DNA condensation occurs locally without direct contact to cationic compaction agents. The consistency of results obtained from DNA complexes with three different types of dendrimers suggests that the observed DNA compaction mechanism at low N/P ratios is a general phenomenon and may also exist in biological cells.

PAMAM dendrimers generation 6 have dimensions and charges comparable to those of the histone core found in chromosomal DNA packing. The observed interactions with this dendrimer of increased size and charge are in strong contrast to those of DNA and smaller cations. Just as in the case of protein folding, studying PAMAM 6/DNA complex formation in microflow reveals a DNA condensation process that exhibits multistage dynamics. Consistently, interaction kinetics at high $\mathrm{pH}$ conditions $(\mathrm{pH}=8.5)$ are found to be particularly slow and the organization of PAMAM 6/DNA in a highly ordered $3 \mathrm{D}$ hexagonal lattice takes place on remarkably long time scales of years.

The principle organization of the DNA chain on the PAMAM 6 surface includes local wrapping of DNA around the dendrimers and is primarily determined by the $\mathrm{pH}$ dependent dendrimer valency. Increasing dendrimer charge density from $1.46 \mathrm{e}^{+} / \mathrm{nm}^{2}$ 
$(\mathrm{pH}=8.5)$ to about $1.7 \mathrm{e}^{+} / \mathrm{nm}^{2}(\mathrm{pH}=5.5)$ results in a transition from a state, where only a finite length of DNA is adsorbed on the dendrimer surface in approximately half of a turn to a full wrapping of DNA in approximately two turns. The cylindrically shaped, fully wrapped conformation of PAMAM 6/DNA entities at low $\mathrm{pH}$ is strikingly similar in shape and size to the structure of nucleosome core particles (NCPs). In this sense, PAMAM 6/DNA entities are excellent biomimetics of NCPs. Consequently, one may conclude that at least for a simple replication of the beads on a string structure without paying attention to DNA functionality, a specific arrangement of charged patches on the histone octamer is not required. The uniform spherical charge distribution of the "artificial protein" results in a structurally analogous packing of DNA. An important point is that for PAMAM 6/DNA entities, connecting (linker-)DNA strands are still present in the structure. Therefore, the PAMAM 6/DNA system represents an ideal starting point towards an experimental realization of the $30 \mathrm{~nm}$ fiber (e.g. by adding linker-histones).

The importance of investigations of DNA condensates is not only relevant from a fundamental point of view. These structures are also extensively used for the delivery of therapeutic genes to living cells. Owing to the increased fraction of DNA, which is tightly bound, the wrapping scenario provides a plausible rationale for the increased stability of DNA complexes with higher generation dendrimers against nuclease digestion. This is a predominant issue reported in literature. Following this line of argumentation, low $\mathrm{pH}$ conditions are better suited for the purpose of DNA delivery inside cells since a larger fraction of DNA is wrapped around the dendrimer. In this scenario, the DNA is also protected. Therefore, results presented in this thesis are believed to contribute in the field of biotechnology towards the construction of new vectors for DNA gene delivery.

In addition to dendrimers, linker-histone $\mathrm{H} 1$ proteins are also used to compact DNA. Exploiting the powerfull combination of X-ray microdiffraction and microfluidics allows for the first time to gain access to H1/DNA structure formation dynamics. Observed SAXS patterns clearly show that the interaction of $\mathrm{H} 1$ with DNA is a two step process: an initial unspecific binding of $\mathrm{H} 1$ proteins to DNA is followed by a rearrangement of molecules in the complexes. Results suggest that the conformational transition of the linker-histone tails from their rather extended conformation in aqueous solution to their fully folded state upon interaction with DNA is responsible for the conformational phase transition of H1/DNA complexes. This kind of rearrangement is most likely necessary to determine the curvature and the path of initial parts of linkerDNA at the entry and exit points of the nucleosome.

The systems studied in this thesis are inherently significant to the fields of biology and biotechnology. The results reported here are expected to have a direct bearing on the 
10. Summary

understanding of chromatin fiber folding into higher order structures. The underlying concepts and techniques may be generalized and used to experimentally access additional relevant biophysical problems and to realize new biomimetic systems. 
11. Appendix

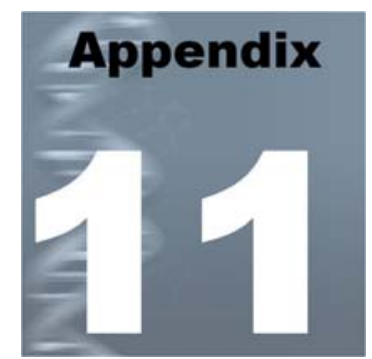

\section{APPENDIX}

\subsection{List of Abbreviations}

A

$A_{\mathrm{P} 6 \mathrm{C}}$

$A_{\text {shape }}$

$a$

$a_{1,} a_{2}$

$\bar{a}_{1}, \bar{a}_{2}$

$a_{\mathrm{H}}$

$a_{\mathrm{O}}$

$a_{\text {rel }}$

$b$

$b_{\mathrm{O}}$

$c_{\mathrm{O}}$ adenine

scattering amplitude

dendrimer surface are covered by DNA

overall shape contribution to the scattering

amplitude

acceleration

fit parameters

fit parameters

hexagonal lattice spacing

orthorhombic unit cell parameter

relative weighing factor

contour length of a curved microchannel

orthorhombic unit cell parameter

orthorhombic unit cell parameter 
11. Appendix

\begin{tabular}{|c|c|}
\hline$c$ & concentration \\
\hline$c_{\text {main }}$ & concentration of the main stream \\
\hline$c_{\text {side }}$ & concentration of the side stream \\
\hline$c_{\mathrm{S}}$ & salt concentration \\
\hline$c_{\mathrm{H}}$ & hexagonal lattice spacing \\
\hline$C$ & cytosine \\
\hline$d$ & spacing/distance \\
\hline$d_{\mathrm{DNA}}$ & average distance between DNA molecules \\
\hline$d_{\mathrm{N}}$ & diameter of the in-house laser beam \\
\hline$d_{\mathrm{S}}$ & diameter of the synchrotron laser beam \\
\hline$d_{\mathrm{DNA}}$ & diameter of the DNA \\
\hline$D e$ & Deborah number \\
\hline$D_{\max }$ & maximum particle extension \\
\hline E & electric field \\
\hline$E_{0}$ & amplitude of the electric field \\
\hline$E_{b}$ & bending energy \\
\hline$e$ & Euler number \\
\hline$e^{+}, e^{-}$ & elementary charge \\
\hline \multirow[t]{2}{*}{$f$} & focal distance \\
\hline & body force density \\
\hline$f_{\mathrm{b}}$ & functionality of dendrimer branches \\
\hline$f_{\mathrm{c}}$ & functionality of the dendrimer core \\
\hline$F$ & force \\
\hline$F$ & single particle scattering function, form factor \\
\hline$F_{\text {shape }}$ & scattering contribution of the overall shape \\
\hline \multirow[t]{2}{*}{$F_{\text {blob }}$} & scattering contribution of internal density \\
\hline & fluctuations \\
\hline \multirow[t]{2}{*}{$G$} & guanine \\
\hline & number of generations \\
\hline \multirow[t]{2}{*}{$h$} & Planck constant \\
\hline & microchannel height \\
\hline$H$ & height of a particle \\
\hline
\end{tabular}


11. Appendix

\begin{tabular}{|c|c|}
\hline$I$ & (scattering) intensity \\
\hline$I_{\text {Anti-Stokes }}$ & intensity of the Anti-Stokes scattering \\
\hline$I_{\text {Stokes }}$ & intensity of the Stokes scattering \\
\hline$I_{\mathrm{GC}}$ & scattering intensity with glassy carbon \\
\hline$j$ & diffusion flux \\
\hline$k$ & Boltzmann factor \\
\hline$l$ & typical length scale of a system \\
\hline$l_{\mathrm{B}}$ & Bjerrum length \\
\hline$L_{\mathrm{a}}$ & adsorbed DNA length \\
\hline$L_{\mathrm{C}}$ & correlation length \\
\hline$L_{\mathrm{P}}$ & persistence length \\
\hline$m$ & mass \\
\hline$M_{\mathrm{w}}$ & molecular weight \\
\hline \multirow[t]{2}{*}{$N / P$} & number of positively charged amino groups divided \\
\hline & $\begin{array}{l}\text { by the number of negatively charge phosphate } \\
\text { groups }\end{array}$ \\
\hline$N$ & number of monomers/atoms/particles \\
\hline$N_{\text {tot }}$ & total number of segments \\
\hline$N_{\text {blob }}$ & number of blobs \\
\hline$N_{\text {seg }}$ & number of segments per blob \\
\hline$N_{\mathrm{S}}$ & number of Shannon channels \\
\hline$O$ & surface area \\
\hline \multirow[t]{3}{*}{$p$} & dipole moment \\
\hline & pressure \\
\hline & pair distance distribution function \\
\hline$P$ & molecular chain length \\
\hline$q$ & momentum transfer, scattering vector \\
\hline $\bar{q}$ & modified scattering vector \\
\hline$q_{\min }$ & minimal $q$-value \\
\hline$q_{\max }$ & maximal $q$-value \\
\hline$Q_{\max }$ & $\begin{array}{l}\text { maximum dendrimer charge assuming complete } \\
\text { protonation }\end{array}$ \\
\hline
\end{tabular}


$Q_{\text {tot }}$

$Q_{\text {I }}$

$Q_{\text {III }}$

$Q_{\text {DNA }}$

$r$

$r_{\min }$

$r_{\max }$

$r_{\mathrm{i}}$

$R$

$R_{\mathrm{P} 6}$

$R_{\mathrm{P} 6 \mathrm{C}}$

$R_{\mathrm{g}}$

$R e$

S

$S_{\mathrm{C}}$

$t$

$T$

u

$u_{0}$

$u_{\text {main }}$

$u_{\text {side }}$

V

$V_{\mathrm{C}}$

$V_{0}$

$V_{\text {water }}$

w

$W i$

$x$

$x_{\max }$

$y$

total dendrimer charge at a certain $\mathrm{pH}$ value

charge of protonated primary amine groups

charge of protonated tertiary amine groups

charge of the (adsorbed) DNA

position of the center of mass

(radial) distance

minimal resolvable length scale

maximal resolvable length scale

position of monomer $i$

radius

radius of PAMAM 6 dendrimers

radius in the PAMAM 6/DNA complex

radius of gyration

Reynolds number

interparticle scattering function, structure factor

unit cell cross section

time

perturbation time constant

temperature

thymine

fluid velocity

initial velocity

velocity of the main stream

velocity of the side stream

volume

unit cell volume

volume of uncharged dendrimers

molecular volume of a water molecule

microchannel width

Weissenberg number

direction in space

position $x$ exhibiting maximal strain rate

direction in space 
11. Appendix

$z$

$\alpha$

$\xi$

$\Delta \chi$

$\Delta q$

$\varepsilon$

$\dot{\varepsilon}$

$\dot{\varepsilon}_{\max }$

$\gamma$

$\eta$

$\eta_{\text {complex }}$

$\eta_{\text {main }}$

$\eta_{\text {side }}$

$\chi$

$\kappa$

$\lambda$

$v$

$\rho$

$\sigma$

$\Sigma$

$\Sigma_{\text {P6 }}$

$\tau$

$\tau_{\mathrm{P}}$

$\tau_{\mathrm{DNA}}$ direction in space

polarizability

aspect ratio

correlation length of density fluctuations

full width at half maximum of an azimuthal intensity distribution

full width at half maximum of a radial intensity distribution

dielectric constant

strain rate

maximal strain rate

density correlation function

viscosity

viscosity of formed complexes

viscosity of the main stream

viscosity of the side stream

azimuthal angle

inverse screening length

wavelength

wave number

Flory-Huggins parameter

density

standard deviation of the Gaussian distribution modeling the surface fuzziness of dendrimers

surface charge density of a dendrimer

surface charge density of PAMAM 6

relaxation time

polymer relaxation time

line charge density of DNA 
11. Appendix

\begin{tabular}{|c|c|}
\hline$\theta$ & scattering angle \\
\hline$\theta_{\mathrm{a}}$ & advancing contact angle \\
\hline$\omega_{0}$ & frequency \\
\hline$\omega_{\mathrm{vib}}$ & vibrational frequency \\
\hline $8 \mathrm{CB}$ & n-octyl-4-cyanobephenyl \\
\hline APD & avalanche photo diode \\
\hline CRL & compound refractive lense \\
\hline CRYSOL & program used to determine $I(q)$ from PDB data \\
\hline DAMMIN & $\begin{array}{l}\text { program used for ab initio modeling scattering } \\
\text { entities }\end{array}$ \\
\hline DNA & deoxyribonucleic acid \\
\hline ESRF & European Synchrotron Radiation Facility \\
\hline $\mathrm{GC}$ & glassy carbon \\
\hline GNOM & program used to calculate $p(r)$ \\
\hline $\mathrm{H} 1, \mathrm{H} 1^{\circ}, \mathrm{H} 5$ & linker-histones \\
\hline $\mathrm{H} 2 \mathrm{~A}, \mathrm{H} 2 \mathrm{~B}, \mathrm{H} 3, \mathrm{H} 4$ & core histones \\
\hline IFT & indirect Fourier transformation \\
\hline KSK & Kapton-Steel-Kapton \\
\hline $\mathrm{NCP}$ & nucleosome core particle \\
\hline PAMAM & poly(amido-amine) \\
\hline PDB & protein data base \\
\hline PDMS & poly(dimethylsiloxane) \\
\hline PMMA & poly(methylmethacrylate) \\
\hline PPI & poly(propylene-imine) \\
\hline RASMOL & program used to display DAMMIN models \\
\hline SAS & small angle scattering \\
\hline SANS & small angle neutron scattering \\
\hline SAXS & small angle $\mathrm{X}$-ray scattering \\
\hline
\end{tabular}




\subsection{Bibliography}

(1) Bloomfield, V. A. (1997). "DNA condensation by multivalent cations. [Review]." Biopolymers, 44, 269.

(2) WAGNER, K.;HARRIES, D. et al. (2000). "Direct evidence for counterion release upon cationic lipid-DNA condensation." Langmuir, 16, 303.

(3) Melnikov, S. M.;SERgeyev, V. G.;Yoshikawa, K.;TAKahashi, H. \& Hatta, I. (1997). "Cooperativity or phase transition - unfolding transition of DNA cationic surfactant complex." Journal of Chemical Physics, 107, 6917.

(4) RAY, J. \& MANNING, G. S. (1999). "Counterion and colon distribution functions in the counterion condensation theory of polyelectrolytes." Macromolecules, 32, 4588.

(5) RAU, D. C. \& PARSEGIAN, V. A. (1992). "Direct measurement of the intermolecular forces between counterion-condensed DNA double-helices. Evidence for long range attractive hydration forces." Biophysical Journal, 61, 245.

(6) Radler, J. O.;Koltover, I.;SAlditt, T. \& SAFinya, C. R. (1997). "Structure of DNA-cationic liposome complexes - DNA intercalation in multilamellar membranes in distinct interhelical packing regimes." Science, 275, 810.

(7) Netz, R. R. \& JoAnNy, J. F. (1998). "Complexation behavior of polyampholytes and charged objects." Macromolecules, 31, 5123.

(8) Raspaud, E.;Delacruz, M. O.;Sikorav, J. L. \& Livolant, F. (1998). "Precipitation of DNA by polyamines - a polyelectrolyte behavior." Biophysical Journal, 74, 381.

(9) SCHIESSEL, H. (2003). "The physics of chromatin [Review]." Journal of Physics-Condensed Matter, 15, R699.

(10) Zinchenko, A. A. \& CHEN, N. (2006). "Compaction of DNA on nanoscale three-dimensional templates [Review]." Journal of Physics-Condensed Matter, 18, R453.

(11) Bohrmann, B.;Haider, M. \& Kellenberger, E. (1993). "Concentration evaluation of chromatin in unstained resin-unbedded sections by means of low-dose ratio-contrast imaging in STEM." Ultramicroscopy, 49, 235.

(12) Morita, M.;TASAKA, M. \& FujISAwA, H. (1993). "DNA packaging ATPase of bacteriophage T3." Virology, 193, 748.

(13) JÜliChER, F.;AJdARI, A. \& PROST, J. (1997). "Modeling molecular motors." Reviews of Modern Physics, 69, 1269. 
(14) Wolf, S. G.;FrENKIEL, D. et al. (1999). "DNA protection by stress-induced biocrystallization." Nature, $\mathbf{4 0 0}, 83$.

(15) Minsky, A.;Shimoni, E. \& FrenkiEL-Krispin, D. (2002). "Stress, order and survival [Review]." Nature Reviews Molecular Cell Biology, 3, 50.

(16) Grant, R. A.;Filman, D. J.;Finkel, S. E.;Kolter, R. \& Hogle, J. M. (1998). "The crystal structure of DPS, a Ferritin homolog that binds and protects DNA." Nature Structural Biology, 5, 294.

(17) KamasheV, D. \& Rouviere-Yaniv, J. (2000). "The histone-like protein HU binds specifically to DNA recombination and repair intermediates." EMBO Journal, 19, 6527.

(18) Wojtuszewski, K.;HAwkins, M. E.;Cole, J. L. \& MukeRJi, I. (2001). "HU binding to DNA: Evidence for multiple complex formation and DNA bending (vol 40, pg 2588, 2001)." Biochemistry, 40, 4892.

(19) WIDOM, J. (1986). "Physicochemical studies of the folding of the $100 \mathrm{~A}$ nucleosome filament into the 300 A filament. Cation dependence." Journal of Molecular Biology, 190, 411.

(20) WidOM, J. (1998). "Structure, dynamics, and functions of chromatin in vitro. [Review]." Annual Review of Biophysics \& Biomolecular Structure, 27, 285.

(21) KoRnBERG, R. D. (1974). "Chromatin structure: a repeating unit of histones and DNA." Science, 184, 868.

(22) Richmond, T. J. \& DAVEY, C. A. (2003). "The structure of DNA in the nucleosome core." Nature, 423, 145.

(23) Wolffe, A. P. \& Pruss, D. (1996). "Chromatin - hanging on to histones." Current Biology, 6, $234 \mathrm{ff}$.

(24) VAn Holde, K. E. (1989). "Chromatin." New York, Springer Verlag.

(25) Woodcock, C. L.;Skoultchi, A. I. \& FAN, Y. H. (2006). "Role of linkerhistone in chromatin structure and function: H1 stoichiometry and nucleosome repeat length." Chromosome Research, 14, 17.

(26) Ruiz-ChicA, J.;MEdina, M. A.;SANCHEZ-JiMENEZ, F. \& RAMiREZ, F. J. (2001). "Fourier transform Raman study of the structural specificities on the interaction between DNA and biogenic polyamines." Biophysical Journal, 80, 443.

(27) McMurry, L. M. \& AlgranATi, I. D. (1986). "Effects of polyamines on trnaslation fidelity in vivo." European Journal of Biochemistry, 155, 383.

(28) Thomas, T. \& Thomas, T. J. (2001). "Polyamines in cell growth and cell death: molecular mechanisms and therapeutic applications [Review]." Cellular \& Molecular Life Sciences, 58, 244. 
(29) TSUCHIDA, E. (1994). "Formation of polyelectrolyte complexes and their structures." Journal of Macromolecular Science-Pure \& Applied Chemistry, A31, 1.

(30) KoetZ, J.;KoepKe, H.;SchmidtnaAKe, G.;Zarras, P. \& Vogl, O. (1996). "Polyanion-Polycation Complex Formation As A Function Of The Position Of The Functional Groups." Polymer, 37, 2775.

(31) VÖGTLE, F.;Buhleier, E. \& WeHner, W. (1978). "Cascade- and nonskid-chainlike syntheses of molecular cavity topologies." Synthesis, 1978, 155.

(32) Tomalia, D. A.;BAKer, H. et al. (1985). Polym. J., 17, 117.

(33) Tomalia, D. A. \& Durst, H. W. (1993). "Supramolecular chemistry I-directed synthesis and molecular recognition." Topics in Current Chemistry, pp. 193.

(34) Jansen, J.;Debrabandervandenberg, E. M. M. \& MeiJer, E. W. (1994). "Encapsulation of guest molecules into a dendritic box." Science, 266, 1226.

(35) Lorenz, K.;Holter, D.;Stuhn, B.;Mulhaupt, R. \& Frey, H. (1996). "A mesogen-functionalized carbosilane dendrimer - a dendritic liquid-crystalline polymer." Advanced Materials, 8, $414 \mathrm{ff}$.

(36) CAKara, D.;Kleimann, J. \& Borkovec, M. (2003). "Microscopic protonation equilibria of poly(amidoamine) dendrimers from macroscopic titrations." Macromolecules, 36, 4201.

(37) Vanduijvenbode, R. C.;Borkovec, M. \& Koper, G. J. M. (1998). "Acid-Base Properties Of Poly(Propylene Imine) Dendrimers." Polymer, 39, 2657.

(38) NisAto, G.;IVKOV, R. \& AmIS, E. J. (2000). "Size invariance of polyelectrolyte dendrimers." Macromolecules, 33, 4172.

(39) Ballauff, M. \& LiKos, C. N. (2004). "Dendrimers in solution: Insight from theory and simulation [Review]." Angewandte Chemie-International Edition, 43, 2998.

(40) Lee, I.;Athey, B. D.;WetZel, A. W.;Meixner, W. \& BAKeR, J. R. (2002). "Structural molecular dynamics studies on polyamidoamine dendrimers for a therapeutic application: Effects of $\mathrm{pH}$ and generation." Macromolecules, 35, 4510.

(41) Lee, C. C.;MacKay, J. A.;Frechet, J. M. J. \& SzokA, F. C. (2005). "Designing dendrimers for biological applications [Review]." Nature Biotechnology, 23, 1517.

(42) Chen, W.;Tomalia, D. A. \& Thomas, J. L. (2000). "Unusual pH-dependent polarity changes in PAMAM dendrimers: Evidence for $\mathrm{pH}$-responsive conformational changes." Macromolecules, 33, 9169.

(43) Maiti, P. K.;CAgin, T.;Lin, S. T. \& Goddard, W. A. (2005). "Effect of solvent and $\mathrm{pH}$ on the structure of PAMAM dendrimers." Macromolecules, 38, 979.

(44) Braun, C. S.;VETRO, J. A. et al. (2005). "Structure/function relationships of polyamidoamine/DNA dendrimers as gene delivery vehicles." J Pharm Sci, 94, 423. 
(45) Bielinska, A. U.;Chen, C. L.;JOHnson, J. \& BAKER, J. R. (1999). "DNA complexing with polyamidoamine dendrimers: Implications for transfection."

Bioconjugate Chemistry, 10, 843.

(46) Tomalia, D. A.;Reyna, L. A. \& Svenson, S. (2007). "Dendrimers as multipurpose nanodevices for oncology drug delivery and diagnostic imaging." Biochemical Society Transactions 35(Part, 35, 61.

(47) KubasiaK, L. A. \& TOMAlia, D. A. (2005). "Cationic dendrimers as gene transfection vectors: Dendri-poly(amidoamines) and dendri-poly(propylenimines)." in: Amiji, M. M. (Ed.) Polymeric Gene Delivery: Principles And Applications, pp. 133 (Boca Raton, CRC press).

(48) Esfand, R. \& TOMALIA, D. A. (2001). "Poly(amidoamine) (PAMAM) dendrimers: from biomimicry to drug delivery and biomedical applications [Review]." Drug Discovery Today, 6, 427.

(49) BielinsKA, A. U.;YEN, A. et al. (2000). "Application of membrane-based dendrimer/DNA complexes for solid phase transfection in vitro and in vivo." Biomaterials, 21, 877.

(50) WANG, Y. O.;Boros, P. et al. (2000). "DNA/dendrimer complexes mediate gene transfer into murine cardiac transplants ex vivo." Molecular, 2, 602.

(51) Roessler, B. J.;BielinsKa, A. U.;JANCZAK, K.;LEe, I. \& BAKER, J. R. (2001). "Substituted beta-cyclodextrins interact with PAMAM dendrimer-DNA complexes and modify transfection efficiency." Biochemical \& Biophysical Research Communications, 283, 124.

(52) WANG, Y. N.;BAI, Y. L. et al. (2001). "Combination of electroporation and DNA/dendrimer complexes enhances gene transfer into murine cardiac transplants." American Journal of Transplantation, 1, 334.

(53) Evans, H. M.;AHMAD, A. et al. (2003). "Structural polymorphism of DNAdendrimer complexes." Physical Review Letters, 9107, 5501.

(54) LIU, Y. C.;CHEN, H. L. et al. (2005). "Mesomorphic complexes of poly(amidoamine) dendrimer with DNA." Macromolecules, 38, 9434.

(55) OtTaViani, M. F.;SACCHI, B. et al. (1999). "An EPR study of the interactions between starburst dendrimers and polynucleotides." Macromolecules, 32, 2275.

(56) Pfohl, T.;Mugele, F.;Seemann, R. \& Herminghaus, S. (2003). "Trends in microfluidics with complex fluids." Chemphyschem, 4, 1291.

(57) Dootz, R. (2004). "Raman-Spektroskopie an mikrostrukturierten Polyelektrolyt-Multischichten." Angewandte Physik, Universität Ulm, Ulm. 
(58) Dootz, R.;Nie, J. J.;Du, B. Y.;Herminghaus, S. \& Pfohl, T. (2006). "Raman and surface enhanced Raman microscopy of microstructured polyethylenimine/DNA multilayers." Langmuir, 22, 1735.

(59) Dootz, R.;OtTen, A.;Koster, S.;Struth, B. \& Pfohl, T. (2006). "Evolution of DNA compaction in microchannels." Journal of Physics-Condensed Matter, 18, S639.

(60) Otten, A.;Koster, S.;Struth, B.;Snigirev, A. \& Pfohl, T. (2005).

"Microfluidics of soft matter investigated by small-angle X-ray scattering." Journal of Synchrotron Radiation 12(Part, 12, 745.

(61) Pfohl, T.;OtTEN, A. et al. (2007). "Highly packed and oriented DNA mesophases identified using in situ microfluidic X-ray microdiffraction." Biomacromolecules, 11, 256.

(62) Struth, B.;SnigireV, A. et al. (2003). "Application of microfocusing at a nonspecific beamline." AIP Conf. Proc. SRI, 705, 804.

(63) Dootz, R.;Evans, H.;Koster, S. \& PFOHL, T. (2007). "Rapid prototyping of Xray microdiffraction compatible continuous microflow foils." Small, 3, 96.

(64) BurghardT, W. R.;Brown, E. F.;AuAd, M. L. \& Kornfield, J. A. (2005). "Molecular orientation of a commercial thermotropic liquid-crystalline polymer in simple shear and complex flow." Rheologica Acta, 44, 446.

(65) RAMAN, C. V. \& KRishnan, K. S. (1928). "A new type of secondary radiation." Nature, 121, 501.

(66) Schrader, B. (1995). "Infrared and Raman spectroscopy." New York, VCH Publishers Inc..

(67) Benevides, J. M.;Overman, S. A. \& Thomas, G. J. (2005). "Raman, polarized Raman and ultraviolet resonance Raman spectroscopy of nucleic acids and their complexes [Review]." Journal of Raman Spectroscopy, 36, 279.

(68) SchUlZ, H. \& BARANSKA, M. (2007). "Identification and quantification of valuable plant substances by IR and Raman spectroscopy [Review]." Vibrational Spectroscopy, 43, 13.

(69) Guinier, A. \& Fournet, G. (1955). "Small angle scattering of X-rays." New York, Wiley.

(70) GlatTER, O. \& KRATKY, O. (1982). "Small angle X-rays scattering." (London, Academic Press).

(71) Als-NiELSEN, J. \& MCMORROW, D. (2001). "Elements of modern X-ray physics." Chichester, John Wiley \& Sons Ltd..

(72) SHANNON, C. E. \& WEAVER, W. (1949). "The mathematical theory of communication." Urbana, IL., University of Illinois Press. 
(73) TAUPIN, D. \& LUZZATI, V. (1982). "Informational content and retrieval in solution scattering studies. I. Degrees of Freedom and data reduction." Journal of Applied Crystallography, 15, 289.

(74) Moore, P. B. (1980). "Small-anlge scattering. Information content and error analysis." Journal of Applied Crystallography, 13, 168.

(75) Svergun, D. I. (1999). "Restoring low resolution structure of biological macromolecules from solution scattering using simulated annealing." Biophysical Journal, 77, 2896.

(76) SEMENYUK, A. V. \& SVERGUN, D. (1991). "GNOM - a program package for small-angle scattering data processing." Journal of Applied Crystallography, 24, 537.

(77) KitTEL, C. (2005). "Introduction to solid state physics." New York, John Wiley \& Sons.

(78) SnigireV, A.;KoHn, V.;SnigireVA, I. \& Lengeler, B. (1996). "A compound refractive lens for focusing high-energy X-rays." Nature, 384, 49.

(79) FIT2D: http://www.esrf.eu/computing/scientific/FIT2D/.

(80) Petoukhov, M. \& Svergun, D. (2007). "DAMMIN."

(81) Svergun, D.;BARBERATO, C. et al. (2007). "CRYSOL."

(82) SCHNITZLER, W. (2004). "Abbildung von Biomolekülen im kontinuierlich Fluss." Angewandte Physik Ulm, University of Ulm.

(83) Beebe, D.;WheEler, M.;Zeringue, H.;WAlters, E. \& RATy, S. (2002). "Microfluidic technology for assisted reproduction." Theriogenology, 57, 125.

(84) Hansen, C. \& QuAKe, S. R. (2003). "Microfluidics in structural biology: smaller, faster. better [Review]." Current Opinion in Structural Biology, 13, 538.

(85) Song, H.;BRinger, M. R.;TICE, J. D.;GERDTS, C. J. \& IsMAgILOV, R. F. (2003). "Experimental test of scaling of mixing by chaotic advection in droplets moving through microfluidic channels." Applied Physics Letters, 83, 4664.

(86) Whitesides, G. M. (2003). "The 'right' size in nanobiotechnology." Nature Biotechnology, 21, 1161.

(87) Zheng, B.;Tice, J. D. \& Ismagilov, R. F. (2004). "Formation of droplets of in microfluidic channels alternating composition and applications to indexing of concentrations in droplet-based assays." Analytical Chemistry, 76, 4977.

(88) Voldman, J.;GRAY, M. L. \& SchMidT, M. A. (1999). "Microfabrication in biology and medicine [Review]." Annual Review of Biomedical Engineering, 1, 401. 
(89) Köster, S.;Steinhauser, D. \& Pfohl, T. (2005). "Brownian motion of actin filaments in confining microchannels." Journal of Physics-Condensed Matter 17(49 Special Issue, 17, S4091.

(90) Guyon, E.;Hulin, J.-P.;Petit, L. \& Mitescu, C. D. (2001). "Physical hydrodynamics." New York, Oxford University Press.

(91) SQuires, T. M. \& QuAKe, S. R. (2005). "Microfluidics: Fluid physics at the nanoliter scale [Review]." Reviews of Modern Physics, 77, 977.

(92) Brody, J. P.;YAger, P.;Goldstein, R. E. \& Austin, R. H. (1996).

"Biotechnology at low Reynolds numbers." Biophysical Journal, 71, 3430.

(93) BAUdRY, J.;CHARlaiX, E.;TONCK, A. \& MAZUYER, D. (2001). "Experimental evidence for a large slip effect at a nonwetting fluid-solid interface." Langmuir, 17, 5232.

(94) Tretheway, D. C. \& MeInhart, C. D. (2002). "Apparent fluid slip at hydrophobic microchannel walls." Physics of Fluids, 14, L9.

(95) Neto, C.;Evans, D. R.;Bonaccurso, E.;Butt, H. J. \& Craig, V. S. J. (2005). "Boundary slip in Newtonian liquids: a review of experimental studies [Review]." Reports on Progress in Physics, 68, 2859.

(96) Cottin-Bizonne, C.;BArRat, J. L.;Bocquet, L. \& CharlaiX, E. (2003). "Low-friction flows of liquid at nanopatterned interfaces." Nature Materials, 2, 237.

(97) Cottin-Bizonne, C.;JuRINE, S. et al. (2002). "Nanorheology: An investigation of the boundary condition at hydrophobic and hydrophilic interfaces." European Physical Journal E, 9, 47.

(98) FurUKAWA, A. \& TANAKA, H. (2006). "Violation of the incompressibility of liquid by simple shear flow." Nature, $443,434$.

(99) Müller, S. J.;LARson, R. G. \& Shaqfeh, E. S. G. (1989). "A purely elastic transition in the Taylor-Couette flow." Rheol. Acta, 28, 499.

(100) Groisman, A. \& Steinberg, V. (2000). "Elastic turbulence in a polymer solution flow." Nature, 405, 53.

(101) BEEBE, D. J.;MoORE, J. S. et al. (2000). "Functional hydrogel structures for autonomous flow control inside microfluidic channels." Nature, 404, 588.

(102) Bird, R. B.;Curtiss, C.;ARmstrong, R. C. \& Hassager, O. (1987). "Dynamics of polymer liquid." New York, Wiley.

(103) Knight, J. B.;Vishwanath, A.;Brody, J. P. \& Austin, R. H. (1998). "Hydrodynamic focusing on a silicon chip - mixing nanoliters in microseconds." Physical Review Letters, 80, 3863. 
(104) KÖSTER, S.;LEACh, J. B.;Struth, B.;Pfohl, T. \& WOnG, J. Y. (2007).

"Visualization of flow-aligned type I collagen self-assembly in tunable $\mathrm{pH}$ gradients." Langmuir, 23, 357.

(105) Pollack, L.;TATE, M. W. et al. (1999). "Compactness of the denatured state of a fast-folding protein measured by submillisecond small-angle x-ray scattering." Proceedings of the National Academy of Sciences of the United States of America, 96, 10115.

(106) Stone, H. A.;STROOCK, A. D. \& AJdARI, A. (2004). "Engineering flows in small devices: Microfluidics toward a lab-on-a-chip [Review]." Annual Review of Fluid Mechanics, 36, 381.

(107) Pollack, L.;TATE, M. W. et al. (2001). "Time resolved collapse of a folding protein observed with small angle x-ray scattering." Physical Review Letters, 86, 4962.

(108) BARRETT, R.;FAUCON, M. et al. (2006). "X-ray microfocussing combined with microfluidics for on-chip X-ray scattering measurements." Lab on a Chip, 6, 494.

(109) HARRISON, D. J.;FLURI, K. et al. (1993). "Micromachining a miniaturized capillary electrophoresis-based chemical analysis system on a chip." Science, 261, 895.

(110) Beebe, D. J.;Mensing, G. A. \& Walker, G. M. (2002). "Physics and applications of microfluidics in biology [Review]." Annual Review of Biomedical Engineering, 4, 261.

(111) NG, J. M. K.;Gitlin, I.;STroock, A. D. \& Whitesides, G. M. (2002). "Components for integrated poly(dimethylsiloxane) microfluidic systems [Review]." Electrophoresis, 23, 3461.

(112) Whitesides, G. M.;Ostuni, E.;TAKayama, S.;Jiang, X. Y. \& IngBer, D. E. (2001). "Soft lithography in biology and biochemistry [Review]." Annual Review of Biomedical Engineering, 3, 335.

(113) DufFy, D. C.;McDonald, J. C.;Schueller, O. J. A. \& Whitesides, G. M. (1998). "Rapid prototyping of microfluidic systems in poly(dimethylsiloxane)." Analytical Chemistry, 70, 4974.

(114) QuAKE, S. R. \& SCHERER, A. (2000). "From micro- to nanofabrication with soft materials [Review]." Science, 290, 1536.

(115) Chaudhury, M. K. \& Whitesides, G. M. (1992). "Correlation between surface free energy and surface constitution." Science, 255, 1230.

(116) McDonald, J. C.;Metallo, S. J. \& Whitesides, G. M. (2001). "Fabrication of a configurable, single-use microfluidic device." Analytical Chemistry, 73, 5645.

(117) Greaves, E. D. \& MANZ, A. (2005). "Toward on-chip X-ray analysis." Lab on a Chip, 5, 382. 
(118) Chandrasekhar, S. (1992). "Liquid crystals." Cambridge, Cambridge University Press.

(119) Gray, G. W. (1974). "Liquid." New York, Plenum Press.

(120) PfOHL, T.;KIM, J. H. et al. (2001). "Controlled modification of microstructured silicon surfaces for confinement of biological macromolecules and liquid crystals." Langmuir, 17, 5343.

(121) CHOI, M. C.;Pfohl, T. et al. (2004). "Ordered patterns of liquid crystal toroidal defects by microchannel confinement." Proceedings of the National Academy of Sciences of the United States of America, 101, 17340.

(122) SAFinYA, C. R.;Sirota, E. B. et al. (1993). "Structure of membrane surfactant and liquid-crystalline smectic lamellar phases under flow." Science, 261, 588.

(123) IdziaK, S. H. J.;Koltover, I.;ISRAelachVili, J. N. \& SAFinya, C. R. (1996). "Structure in a confined smectic liquid crystal with competing surface and sample elasticities." Physical Review Letters, 76, 1477.

(124) IDZIAK, S. H. J.;SAFINYA, C. R. et al. (1994). "The X-ray surface forces apparatus - structure of a thin smectic liquid crystal film under confinement." Science, 264, 1915.

(125) PICTURE TO THE LEFT: WHEELER, R. "http://en.wikipedia.org/wiki/Image:ADNA\%2C_B-DNA_and Z-DNA.png."

(126) WATSON, J. \& CRICK, F. (1953). "Molecular structure of nucleic acids; a structure for deoxyribose nucleic acid." Nature, 171, 737.

(127) Mandelkern, M.;Elias, J. G.;Eden, D. \& Crothers, D. M. (1981). "The dimensions of DNA in solution." Journal of Molecular Biology, 152, 153.

(128) Smith, S. B.;Finzi, L. \& Bustamante, C. (1992). "Direct mechanical measurements of the elasticity of single DNA molecules by using magnetic beads." Science, 258, 1122.

(129) Lodisch, H.;BERK, A. et al. (2000). "Molekulare Zellbiologie." Heidelberg, Berlin, Spektrum Akademischer Verlag.

(130) WING, R.;DREW, H. et al. (1980). "Crystal structure analysis of a complete turn of B-DNA." Nature, 287, 755.

(131) HEO, Y. \& LARSON, R. G. (2005). "The scaling of zero-shear viscosities of semidilute polymer solutions with concentration." Journal of Rheology, 49, 1117.

(132) WANG, L. \& BloOmfield, V. A. (1991). "Small-angle X-ray scattering of semidilute rodlike DNA solutions: polyelectrolyte behavior." Macromolecules, 24, 5791. 
(133) SKIBINSKA, L.;GAPINSKI, J. et al. (1999). "Effect of electrostatic interactions on the structure and dynamics of a model polyelectrolyte. II. Intermolecular correlations." Journal of Chemical Physics, 110, 1794.

(134) Borsali, R.;Nguyen, H. \& PeCORA, R. (1998). "Small-angle neutron scattering and dynamic light scattering from a polyelectrolyte solution - DNA." Macromolecules, 31, 1548 .

(135) Koch, M. H. J.;SAYERS, Z.;SICRE, P. \& SVERGUn, D. (1995). "A synchrotron radiation electric field $X$-ray solution scattering study of DNA at very low ionic strength." Macromolecules, 28, 4904.

(136) Livolant, F. \& Leforestier, A. (1996). "Condensed phases of DNA structures and phase transitions. [Review]." Progress in Polymer Science, 21, 1115.

(137) Castelletto, V.;Itri, R.;Amaral, L. Q. \& Spada, G. P. (1995). "Small-angle X-ray scattering of DNA fragments - form and interference factors." Macromolecules, 28, 8395 .

(138) LeForestier, A. \& Livolant, F. (1993). "Supramolecular ordering of DNA in the cholesteric liquid-crystalline phase: an ultrastructural study." Biophys $J, \mathbf{6 5}, 56$.

(139) Livolant, F.;LeVelut, A. M.;Doucet, J. \& BenOit, J. P. (1989). "The highly concentrated liquid-crystalline phase of DNA is columnar hexagonal." Nature, 339, 724 .

(140) Livolant, F. (1984). "Cholesteric organization of DNA in vivo and in vitro." Eur J Cell Biol, 33, 300.

(141) LePault, J.;Dubochet, J.;BAschong, W. \& Kellenberger, E. (1987). "Organization of double-stranded DNA in bacteriophages: a study by cryo-electron microscopy of vitrified samples." Embo J, 6, 1507.

(142) Feughelman, M.;LANGRIDGe, R. et al. (1955). "Molecular structure of deoxyribose nucleic acid and nucleoprotein." Nature, 175, 834.

(143) Hayter, J.;Jannink, G.;Brochard-Wyart, F. \& DE Gennes, P.-G. (1980). J. Phys. (Paris) Lett., 41, L451.

(144) KoyAma, R. (1984). "Small-angle scattering of polyelectrolyte solutions." Macromolecules, 17, 1594.

(145) Deng, H.;Bloomfield, V. A.;Benevides, J. M. \& Thomas, G. J. (1999). "Dependence of the raman signature of genomic B-DNA on nucleotide base sequence." Biopolymers, 50, 656.

(146) Deng, H.;Bloomfield, V. A.;Benevides, J. M. \& Thomas, G. J. (2000). "Structural basis of polyamine-DNA recognition: spermidine and spermine interactions with genomic B-DNAs of different GC content probed by Raman spectroscopy." Nucleic Acids Research, 28, 3379. 
(147) GuAN, Y. F.;CHOY, G. S. C.;GLASER, R. \& ThOMAS, G. J. (1995). "Vibrational analysis of nucleic acids - 2. Ab initio calculation of the molecular force field and normal modes of dimethyl phosphate." Journal of Physical Chemistry, 99, 12054.

(148) LiU, Y. L.;ZhaO, M. Q.;Bergbreiter, D. E. \& Crooks, R. M. (1997). "pHswitchable, ultrathin permselective membranes prepared from multilayer polymer composites." Journal of the American Chemical Society, 119, 8720.

(149) GU, Y. L.;XIE, H. et al. (2005). "AFM characterization of dendrimer-stabilized platinum nanoparticles." Langmuir, 21, 3122.

(150) ScotT, R. W. J.;Sivadinarayana, C. et al. (2005). "Titania-supported PdAu bimetallic catalysts prepared from dendrimer-encapsulated nanoparticle precursors." Journal of the American Chemical Society, 127, 1380.

(151) MaCKAY, M. E.;HONG, Y. et al. (2002). "Microphase separation of hybrid dendron-linear diblock copolymers into ordered structures." Macromolecules, 35, 8391.

(152) TAnde, B. M.;WAgner, N. J.;Mackay, M. E.;HAwKer, C. J. \& JeONG, M. (2001). "Viscosimetric, hydrodynamic, and conformational properties of dendrimers and dendrons." Macromolecules, 34, 8580.

(153) JIANG, Y. H.;EMAU, P. et al. (2005). "SPL7013 gel as a topical microbicide for prevention of vaginal transmission of SHIV 89.6P in macaques." AIDS Research \& Human Retroviruses, 21, 207.

(154) Frechet, J. M. J.;Hawker, C. J.;Gitsov, I. \& LeOw, J. (1990). Pure Appl. Chem., A33, 1425.

(155) DE GENNES, P.-G. \& HeRvet, H. (1983). "Statistics of starbust polymers." $J$. Physiol. Lett., 44, L351.

(156) Murat, M. \& Grest, G. S. (1996). "Molecular dynamics study of dendrimer molecules in solvents of varying quality." Macromolecules, 29, 1278.

(157) Mansfield, M. L. (2000). "Monte Carlo studies of dendrimers. Additional results for the diamond lattice model." Macromolecules, 33, 8043.

(158) Boris, D. \& RUBinsteIn, M. (1996). "A self-consistent mean field model o a starburst dendrimer - dense core vs dense shell." Macromolecules, 29, 7251.

(159) Prosa, T. J.;BAuer, B. J. \& Amis, E. J. (2001). "From stars to spheres: A SAXS analysis of dilute dendrimer solutions." Macromolecules, 34, 4897.

(160) Rathgeber, S.;Monkenbusch, M.;Kreitschmann, M.;Urban, V. \& Brulet, A. (2002). "Dynamics of star-burst dendrimers in solution in relation to their structural properties." Journal of Chemical Physics, 117, 4047.

(161) Rosenfeldt, S.;Dingenouts, N. et al. (2002). "Determination of the structure factor of polymeric systems in solution by small-angle scattering: A SANS-Study of a dendrimer of fourth generation." Macromolecular Chemistry \& Physics, 203, 1995. 
(162) BoAs, U. \& HeEgaARD, P. M. (2004). "Dendrimers in drug research." Chem Soc Rev, 33, 43.

(163) SchiESSEL, H. \& Pincus, P. (1998). "Counterion-condensation-induced collapse of highly charged polyelectrolytes." Macromolecules, 31, 7953.

(164) Jusufi, A.;Likos, C. N. \& Lowen, H. (2002). "Conformations and interactions of star-branched polyelectrolytes - art. no. 018301." Physical Review Letters, 8801, 8301 .

(165) Maiti, P. K.;CAgin, T.;WAng, G. F. \& Goddard, W. A. (2004). "Structure of PAMAM dendrimers: Generations 1 through 11." Macromolecules, 37, 6236.

(166) Welch, P. \& Muthukumar, M. (1998). "Tuning the density profile of dendritic polyelectrolytes." Macromolecules, 31, 5892.

(167) Prosa, T. J.;BAuer, B. J.;Amis, E. J.;Tomalia, D. A. \& ScherrenberG, R. (1997). "A SAXS study of the internal structure of dendritic polymer systems." Journal of Polymer Science Part B-Polymer Physics, 35, 2913.

(168) Higgins, J. S. \& Benoít, H. C. (1994). "Polymers and neutron scattering." Oxford, Claredon Press.

(169) LI, J.;PIEHLER, L. T. et al. (2000). "Visualization and characterization of poly(amidoamine) dendrimers by atomic force microscopy." Langmuir, 16, 5613.

(170) Dozier, W. D.;HuAng, J. S. \& FetTers, L. J. (1991). "Colloidal nature of star polymer dilute and semi-dilute solution." Macromolecules, 24, 2810.

(171) Beaucage, G. (1995). "Approximations leading to a unified exponential powerlaw approach to small-angle scattering." Journal of Applied Crystallography, 28, 717.

(172) Daoud, M. \& Cotton, J. P. (1982). "Sta shaped polymers." J. Phys. (France), 43,531 .

(173) FlORY, P. J. (1956). "Statistical thermodynamics of semi-flexible chain molecules." Proc. R. Soc. London A, 234, 73.

(174) Rathgeber, S.;PAKula, T. \& URBAn, V. (2004). "Structure of star-burst dendrimers: A comparison between small angle x-ray scattering and computer simulation results." Journal of Chemical Physics, 121, 3840.

(175) Loppinet, B.;Stiakakis, E.;Vlassopoulos, D.;Fytas, G. \& RoOvers, J. (2001). "Reversible thermal gelation in star polymers: An alternative route to jamming of soft matter." Macromolecules, 34, 8216.

(176) RiCHTER, D.;SCHNEIDERS, D. et al. (1997). "Polymer aggregates with crystalline cores - the system polyethylene-poly(ethylenepropylene)." Macromolecules, 30, 1053.

(177) RAMZI, A.;PRAGER, M. et al. (1997). "Influence of polymer architecture on the formation of micelles of miktoarm star copolymers 
polyethylene/poly(ethylenepropylene) in the selective solvent decane."

Macromolecules, 30, 7171.

(178) RAthgeber, S.;GAST, A. P. \& HEDRICK, J. L. (2002). "Structural properties of star-like dendrimers in solution." Applied Physics A, 74, S396.

(179) Gotze, I. O. \& LiKos, C. N. (2003). "Conformations of flexible dendrimers: A simulation study." Macromolecules, 36, 8189.

(180) WiLlNER, L.;JUCKNISCHKE, O. et al. (1994). "Structural investigation of star polymers in solution by small angle neutron scattering." Macromolecules, 27, 3821.

(181) Megens, M.;Vankats, C. M.;Bosecke, P. \& Vos, W. L. (1997). "Synchrotron small-angle X-ray scattering of colloids and photonic colloidal crystals." Journal of Applied Crystallography, 30, 637.

(182) Mallamace, F.;CAnetTA, E. et al. (2002). "Scaling properties in the internal structure of dendrimer systems." Physica A, 304, 235.

(183) Richter, D.;FArago, B.;Fetters, L. J.;Huang, J. S. \& Ewen, B. (1990). "On the relation between structure and dynamics of star polymers in dilute solution." Macromolecules, 23, 1845.

(184) NiU, Y. H.;SUN, L. \& CROOKS, R. A. (2003). "Determination of the intrinsic proton binding constants for poly(amidoamine) dendrimers via potentiometric $\mathrm{pH}$ titration." Macromolecules, 36, 5725.

(185) Uppuluri, S.;Keinath, S. E.;Tomalia, D. A. \& Dvornic, P. R. (1998). "Rheology of dendrimers - I. Newtonian flow behavior of medium and highly concentrated solutions of polyamidoamine (PAMAM) dendrimers in ethylenediamine (EDA) solvent." Macromolecules, 31, 4498.

(186) Betley, T. A.;Holl, M. M. B. et al. (2001). "Tapping mode atomic force microscopy investigation of poly(amidoamine) dendrimers: Effects of substrate and $\mathrm{pH}$ on dendrimer deformation." Langmuir, 17, 2768.

(187) Dvorkin, P. L.;Ryzhova, G. L. \& Lebedev, Y. A. (1983). "Parameters for calculating the molar volumen and density of liquid organic substances." Izvestiya Akademii Nauk SSSR, Seriya Khimicheskaya, 5, 1101.

(188) $\quad$ HTTP://WWW.LSBU.AC.UK/WATER/DATA.HTML.

(189) Boussif, O.;LEzOUALCH, F. et al. (1995). "A versatile vector for gene and oligonucleotide transfer into cells in culture and in vivo - polyethylenimine."

Proceedings of the National Academy of Sciences of the United States of America, 92, 7297.

(190) DeDuve, C.;DeBARsy, T. et al. (1974). "Lysosomotropic agents." Biochem. Pharmacol., 23, 2495. 
(191) Tang, M. X.;Redemann, C. T. \& SzoKa, F. C. (1996). "In Vitro Gene Delivery By Degraded Polyamidoamine Dendrimers." Bioconjugate Chemistry, 7, 703.

(192) Petrov, J. G.;Pfohl, T. \& MöHwald, H. (1999). "Ellipsometric chain length dependence of fatty acid Langmuir monolayers. A heads-and-tails model." Physical Chemistry $B, \mathbf{1 0 3}, 3417$.

(193) Martinez, A. \& Kolter, R. (1997). "Protection Of Dna During Oxidative Stress By The Nonspecific Dna-Binding Protein Dps." Journal of Bacteriology, 179, 5188.

(194) AtKIns, P. W. (1998). "Physical chemistry." Oxford, Oxford University Press.

(195) Koester, S. (2006). "Biological Matter in Microfluidic Enviroment - from Single Molecules to Self-Assembly." MPI for Dynamics and Self-Organization (Göttingen, Georg-August-Universität Göttingen).

(196) EstrofF, L. A.;Leiserowitz, L.;AdDAdi, L.;Weiner, S. \& HAMilton, A. D. (2003). "Characterization of an organic hydrogel: A cryo-transmission electron microscopy and X-ray diffraction study." Advanced Materials, 15, 38.

(197) MuKhopadhyay, S.;MAITRA, U. et al. (2004). "Structure and dynamics of a molecular hydrogel derived from a tripodal cholamide." Journal of the American Chemical Society, 126, 15905.

(198) Szymanski, J.;PAtKowski, A.;Wilk, A.;Garstecki, P. \& Holyst, R. (2006). "Diffusion and viscosity in a crowded environment: From nano- to macroscale." Journal of Physical Chemistry B, 110, 25593.

(199) JENA, S. S. \& BloOMfield, V. A. (2005). "Probe diffusion in concentrated polyelectrolyte solutions: Effect of background interactions on competition between electrostatic and viscous forces." Macromolecules, 38, 10551.

(200) Petit, J. M.;Roux, B.;Zhu, X. X. \& Macdonald, P. M. (1996). "A new physical model for the diffusion of solvents and solute probes in polymer solutions." Macromolecules, 29, 6031.

(201) Petit, J. M.;Zhu, X. X. \& Macdonald, P. M. (1996). "Solute probe diffusion in aqueous solutions of poly(vinyl alcohol) as studied by pulsed-gradient spin-echo NMR spectroscopy." Macromolecules, 29, 70.

(202) Phillies, G. D. J. (1987). "Dynamics of plymers in concentrated solutions: the universal scaling equation derived." Macromolecules, 20, 558.

(203) Phillies, G. D. J. (1989). "The hydrodynamic scaling model for polymer selfdiffusion." Journal of Physical Chemistry, 93, 5029.

(204) MASARO, L.;ZHU, X. X. \& MACDONALD, P. M. (1998). "Self-diffusion of oligoand poly(ethylene glycol)s in poly(vinyl alcohol) aqueous solutions as studied by pulsed-gradient NMR spectroscopy." Macromolecules, 31, 3880. 
(205) Masaro, L.;Zhu, X. X. \& MacDonald, P. M. (1999). "Study of the selfdiffusion of poly(ethylene glycol)s in poly(vinyl alcohol) aqueous systems." Journal of Polymer Science Part B-Polymer Physics, 37, 2396.

(206) Lin, T.-H. \& PHillies, G. D. J. (1984). "Translational diffusion of a macro particulant probe species in salt-free poly(acrylic acid):water." Journal of Colloids and Interface Science, 100, 82.

(207) OosawA, F. (1971). "Polyelectrolytes." New York, Marcel Dekker.

(208) Dunstan, D. E. \& STOKES, J. (2000). "Diffusing probe measurements of polystyrene latex particles in polyelectrolyte solutions: Deviations from StokesEinstein behavior." Macromolecules, 33, 193.

(209) PPI4/DNACOMPLEXES (2004). "X-ray microdiffraction measurements performed by A. Otten, S. Köster, B. Du, T. Pfohl at ESRF (beamline ID10b), data treatment and analysis by R.D."

(210) PARAMETERS: $\mathrm{D}_{\mathrm{PPI} 4} \approx 1.6 \cdot 10^{-10} \mathrm{~m}^{2} \mathrm{~s}^{-1}, \mathrm{D}_{\mathrm{DNA}} \approx 2 \cdot 10^{-12} \mathrm{~m}^{2} \mathrm{~s}^{-1}, \eta_{\mathrm{DNA}} \approx 10^{3} \eta_{\text {water }}$.

(211) Grosberg, A. Y.;Nguyen, T. T. \& Shklovskit, B. I. (2002). "Colloquium: The physics of charge inversion in chemical and biological systems [Review]." Reviews of Modern Physics, 74, 329.

(212) Ghirlando, R.;WAChtel, E. J.;ARAD, T. \& Minsky, A. (1992). "DNA packing induced by micellar aggragates: a novel in vitro DNA condensation system."

Biochemistry, 31, 7110.

(213) BLOOMFIELD, V. A. (1991). "Condensation of DNA by multivalent cations: consideration of mechanism." Biopolymers, 31, 1471.

(214) Evans, H. M. (2005). "Structure-function investigations of DNA condensing agents with application to gene delivery." (Santa Barbara, University of California Santa Barbara).

(215) Pelta, J.;Durand, D.;Doucet, J. \& Livolant, F. (1996). "DNA mesophases induced by spermidine - structural properties and biological implications." Biophysical Journal, 71, 48.

(216) Wolffe, A. (1998). "Chromatin structure and function." San Diego, Academic Press.

(217) PUROHIT, P. K.;INAMDAR, M. M. et al. (2005). "Forces during bacteriophage DNA packaging and ejection." Biophysical Journal, 88, 851.

(218) Mangenot, S.;Leforestier, A.;Durand, D. \& Livolant, F. (2003). "Phase diagram of nucleosome core particles." Journal of Molecular Biology, 333, 907.

(219) Mangenot, S.;Leforestier, A.;Durand, D. \& Livolant, F. (2003). "X-ray diffraction characterization of the dense phases formed by nucleosome core particles." Biophysical Journal, 84, 2570. 
(220) Richmond, T. J.;Finch, J. T.;Rushton, B.;Rhodes, D. \& KLuG, A. (1984). "Strucutre of the nucleosome core particle at 7A resolution." Nature, 311, 532.

(221) White, C. L.;Suto, R. K. \& LugER, K. (2001). "Structure of the yeast nucleosome core particle reveals fundamental changes in internucleosome interactions." EMBO Journal, 20, 5207.

(222) Luger, K.;MAder, A. W.;Richmond, R. K.;SARgent, D. F. \& Richmond, T. J. (1997). "Crystal structure of the nucleosome core particle at 2.8-Angstrom resolution." Nature, 389, 251.

(223) ZHANG, X. M.;Hirota, N. et al. (1999). "Investigation of molecular diffusion in hydrogel by electronic speckle pattern interferometry." Journal of Physical Chemistry $B, \mathbf{1 0 3}, 6069$.

(224) Haronska, P.;Vilgis, T. A.;Grottenmuller, R. \& Schmidt, M. (1998). "Adsorption Of Polymer Chains Onto Charged Spheres - Experiment And Theory." Macromolecular Theory \& Simulations, 7, 241.

(225) Bielinska, A. U.;Kukowskalatallo, J. F. \& BAKER, J. R. (1997). "The interaction of plasmid DNA with polyamidoamine dendrimers - mechanism of complex formation and analysis of alterations induced in nuclease sensitivity and transcriptional activity of the complexed DNA." Biochimica et Biophysica Acta - Gene Structure \& Expression, 1353, 180.

(226) Svenson, S. \& Tomalia, D. A. (2005). "Dendrimers in biomedical applications--reflections on the field." Adv Drug Deliv Rev, 57, 2106.

(227) Sali, A.;Shakhnovich, E. \& Karplus, M. (1994). "How Does A Protein Fold." Nature, 369, 248.

(228) Guinier, A. \& Fournet, G. (1955). "Small angle scattering of X-rays." Wiley, New York.

(229) Svergun, D. I. \& KocH, M. H. J. (2003). "Small-angle scattering studies of biological macromolecules in solution [Review]." Reports on Progress in Physics, 66, 1735 .

(230) Glatter, O. (1977). "A new method for the evaluation of small-angle scattering data." Journal of Applied Crystallography, 10, 415.

(231) MÜLleR, K. \& GLATTER, O. (1982). "Practical aspects to the use of indirect Fourier transformation methods." Makromol. Chem., 183, 465.

(232) Svergun, D. (1992). "Determination of the regularization parameter in indirecttransform methods using perceptual criteria." Journal of Applied Crystallography, 25, 495. 
(233) Pedersen, J. S. \& Schurtenberger, P. (1996). "Cross-section structure of cylindrical and polymer-like micelles from small-angle scattering data. - 1. Test of analysis methods." Journal of Applied Crystallography, 29, 646.

(234) Mangenot, S.;Leforestier, A.;Vachette, P.;Durand, D. \& LiVolant, F. (2002). "Salt-induced conformation and interaction changes of nucleosome core particles." Biophysical Journal, 82, 345.

(235) Svergun, D.;BArberato, C. \& Koch, M. H. J. (1995). "Crysol - A Program To Evaluate X-Ray Solution Scattering Of Biological Macromolecules From Atomic Coordinates." Journal of Applied Crystallography, 28, 768.

(236) HARP, J. M.;HANSON, B. L.;TimM, D. E. \& BuniCK, G. J. (2000). "Asymmetries in the nucleosome core particle at 2.5 angstrom resolution." Acta Crystallographica Section D-Biological Crystallography 56(Part, 56, 1513.

(237) Gelamo, E. L.;ItRI, R. \& TABAK, M. (2004). "Small angle X-ray scattering (SAXS) study of the extracellular hemoglobin of Glossoscolex paulistus - Effect of $\mathrm{pH}$, iron oxidation state, and interaction with anionic SDS surfactant." Journal of Biological Chemistry, 279, 33298.

(238) SAyle, R. \& Bernstein, H. (2004). "RASMOL."

(239) Volkov, V. V. \& SVergun, D. I. (2003). "Uniqueness of ab initio shape determination in small-angle scattering." Journal of Applied Crystallography, 36, 860.

(240) LyUlin, S. V.;DARINSKII, A. A. \& Lyulin, A. V. (2005). "Computer simulation of complexes of dendrimers with linear polyelectrolytes." Macromolecules, 38, 3990.

(241) KunZE, K. K. \& Netz, R. R. (2000). "Salt-induced DNA-histone complexation." Physical Review Letters, 85, 4389.

(242) Chen, W.;Turro, N. J. \& Tomalia, D. A. (2000). "Using ethidium bromide to probe the interactions between DNA and dendrimers." Langmuir, 16, 15.

(243) Netz, R. R. \& JoAnNy, J. F. (1999). "Complexation between a semi-flexible polyelectrolyte and an oppositely charged sphere." Macromolecules, 32, 9026.

(244) LASCHAT, S.;BARO, A. et al. (2007). "Discotic liquid crystals: From tailor-made synthesis to plastic electronics [Review]." Angewandte Chemie-International Edition, 46, 4832 .

(245) Morale, F.;DATE, R. W. et al. (2003). "Columnar mesomorphism from hemidisklike metallomesogens derived from 2,6-bis[3 ',4 ',5'-

tri(alkoxy)phenyliminomethyl]pyridines (L): Crystal and molecular structures of [M(L)Cl-2 (M = Mn, Ni, Zn)." Chemistry-A European Journal, 9, 2484.

(246) Bertin, A.;Renouard, M.;Pedersen, J. S.;Livolant, F. \& DuRAnd, D. (2007). "H3 and H4 histone tails play a central role in the interactions of recombinant NCPs." Biophys $J, 92,2633$. 
(247) Kunze, K. K. \& Netz, R. R. (2002). "Complexes of semi-flexible polyelectrolytes and charged spheres as models for salt-modulated nucleosomal structures - art. no. 011918 [Review]." Physical Review E 6601(1 Part, 6601, 1918.

(248) ARCesi, L.;LA PenNA, G. \& Perico, A. "A generalized electrostatic model of the wrapping of DNA around oppositely charged proteins."

(249) HowARD, J. (2001). "Mechanical of motor proteins and the cytoskeleton." Sinauer Ass. Inc., Sunderland.

(250) OdiJK, T. (1977). Polymer Science, 15, 477.

(251) Nguyen, T. T.;Grosberg, A. Y. \& Shklovskit, B. I. (2000). "Macroions in salty water with multivalent ions: Giant inversion of charge." Physical Review Letters, 85, 1568 .

(252) Kukowskalatallo, J. F.;Bielinska, A. U. et al. (1996). "Efficient Transfer Of Genetic Material Into Mammalian Cells Using Starburst Polyamidoamine Dendrimers." Proceedings of the National Academy of Sciences of the United States of America, 93, 4897.

(253) WidOM, J. (1998). "Chromatin Structure - Linking Structure To Function With Histone H1." Current Biology, 8, R 788.

(254) Zlatanova, J.;CAiAfa, P. \& Van Holde, K. (2000). "Linker-histone binding and displacement: versatile mechanism for transcriptional regulation [Review]." FASEB Journal, 14, 1697.

(255) Georgel, P. T. \& HAnsen, J. C. (2001). "Linker-histone function in chromatin: Dual mechanisms of action [Review]." Biochemistry \& Cell Biology-Biochimie \& Biologie Cellulaire, 79, 313.

(256) Bustin, M.;CATEZ, F. \& LiM, J. H. (2005). "The dynamics of histone H1 function in chromatin." Molecular Cell, 17, 617.

(257) Thomas, J. O. (1999). "Histone H1: location and role." Current Opinion in Cell Biology, 11, 312.

(258) Travers, A. (1999). "The location of the linker-histone on the nucleosome." Trends in Biochemical Sciences, 24, 4.

(259) Harvey, A. C. \& Downs, J. A. (2004). "What functions do linker-histones provide? [Review]." Molecular Microbiology, 53, 771.

(260) KASINSKY, H. E.;LEWIS, J. D.;DACKS, J. B. \& AusiO, J. (2001). "Origin of H1 linker-histones [Review]." FASEB Journal, 15, 34.

(261) SERA, T. \& WolfFe, A. P. (1998). "Role of histone H1 as an architectural determinant of chromatin structure and as a specific repressor of transcription on Xenopus oocyte 5S genes. [Review]." Molecular \& Cellular Biology, 18, 3668. 
(262) CoLE, R. D. (1984). "A minireview of microheterogeneity in H1 histone and its possible significants." Analytical Biochemistry, 136, 24.

(263) Hansen, C. \& Ausio, J. (1992). "Chromatin dynamics and the modulation of genetic activity." Trends in Biochemical Sciences, 17, 187.

(264) Wolffe, A. P.;Khochbin, S. \& Dimitrov, S. (1997). "What do linker-histones do in chromatin?" Bioessays, 19, 249.

(265) CRANE-Robinson, C. (1999). "How do linker-histones mediate differential gene expression?" Bioessays, 21, 367.

(266) Ausio, J. (2000). "Are linker-histones (histone H1) dispensable for survival?" Bioessays, 22, 873.

(267) Neelin, J. M.;Callahan, P. X.;Lamb, D. C. \& Murray, K. (1964). "The histones of chicken erythrocyte nuclei." Canadian Journal of Biochemistry, 42, 1743.

(268) Ausio, J. (1999). "Histone H1 and evolution of sperm nuclear basic proteins [Review]." Journal of Biological Chemistry, 274, 31115.

(269) Aviles, F. J.;Chapman, G. E.;Kneale, G. G.;Crane-Robinson, C. \& BRADBURY, E. M. (1978). "The conformation of histone H5." European Journal of Biochemistry, 88, 363.

(270) RAmakrishnan, V.;Finch, J. T.;GraZiano, V.;LeE, P. L. \& SweEt, R. M. (1993). "Crystal structure of globular domain of histone H5 and its implication for nucleosome binding." Nature, 362, 219.

(271) CERF, C.;LIPPENS, G. et al. (1994). "Homo- and heteronuclear twodDimensional NMR studies of the globular domain of histone H1 - full assignment, tertiary structure, and comparison with the globular domain of histone H5." Biochemistry, 33, 11079.

(272) Mamoon, N. M.;Song, Y. G. \& Wellman, S. E. (2002). "Histone H1 ${ }^{\circ}$ and its carboxyl-terminal domain bind in the major groove of DNA." Biochemistry, 41, 9222.

(273) Goytisolo, F. A.;Gerchman, S. E. et al. (1996). "Identification of two DNAbinding sites on the globular domain of histone H5." EMBO Journal, 15, 3421.

(274) Allan, J.;Hartman, P. G.;Crane-Robinson, C. \& Aviles, F. X. (1980). "The structure of histone $\mathrm{H} 1$ and its location in chromatin." Nature, 288, 675.

(275) Hayes, J. J. \& Wolffe, A. P. (1993). Proceedings of the National Academy of Sciences of the United States of America, 90, 6415.

(276) Zlatanova, J. \& VANHOlde, K. (1998). "Binding to four-way junction DNA a common property of architectural proteins. [Review]." FASEB Journal, 12, 421.

(277) Pruss, D.;BARTholomew, B. et al. (1996). "An asymmetric model for the nucleosome - a binding site for linker-histones inside the DNA gyres." Science, 274, 614. 
(278) Hayes, J. J.;Kaplan, R.;Ura, K.;Pruss, D. \& WolfFe, A. (1996). "A Putative Dna Binding Surface In The Globular Domain Of A Linker-Histone Is Not Essential For Specific Binding To The Nucleosome." Journal of Biological Chemistry, 271, 25817.

(279) ZHOU, Y. B.;GERChMAN, S. E.;RAMAKRISHNAN, V.;TRAVERS, A. \& MUYLDERMANS, S. (1998). "Position and orientation of the globular domain of linkerhistone H5 on the nucleosome." Nature, 395, 402.

(280) VILA, R.;PONTE, I. et al. (2001). "DNA-induced alpha-helical structure in the NH2-terminal domain of histone H1." Journal of Biological Chemistry, 276, 46429.

(281) Vila, R.;Ponte, I.;Collado, M.;Arrondo, J. L. R. \& SuAu, P. (2001). "Induction of secondary structure in a $\mathrm{COOH}$-terminal peptide of histone $\mathrm{H} 1$ by interaction with the DNA - An infrared spectroscopy study." Journal of Biological Chemistry, 276, 30898.

(282) Vila, R.;Ponte, I.;Jimenez, M. A.;Rico, M. \& SuAu, P. (2000). "A helix-turn motif in the C-terminal domain of histone H1." Protein Science, 9, 627.

(283) ClarK, D. J.;Hill, C. S.;Martin, S. R. \& Thomas, J. O. (1988). "Alpha-helix in the carboxy-terminal domains of histones H1 and H5." EMBO Journal, 7, 69.

(284) Hill, C. S.;Martin, S. R. \& Thomas, J. O. (1989). "A stable alpha-helical element in the carboxy-terminal domain of free and chromatin-bound histones H1 from sea urchin sperm." EMBO Journal, 8, 2591.

(285) BÖHM, L. \& CREemers, P. C. (1993). "Protein structure and molecular enzymology." Biochimica et Biophysica Acta, 1202, 230.

(286) CARTER, G. J. \& VAN Holde, K. (1998). "Self-association of linker-histone H5 and of its globular domain - evidence for specific self-contacts." Biochemistry, 37, 12477.

(287) Subirana, J. A. (1990). "Analysis of the charge distribution in the carboxylterminal region of histone $\mathrm{H} 1$ as related to its interaction with DNA." Biopolymers, 29, 1351.

(288) Allan, J.;Mitchell, T.;Harborne, N.;Bohm, L. \& Crane-Robinson, C. (1986). "Roles of H1 domains in determining chromatin strcutre and histone location." Journal of Molecular Biology, 187, 591.

(289) LU, X. \& HANSEN, J. C. (2004). "Identification of specific functional subdomains within the linker-histone H1(o)C-terminal domain." Journal of Biological Chemistry, 279, 8701.

(290) Misteli, T.;Gunjan, A.;Hock, R.;Bustin, M. \& Brown, D. T. (2000). "Dynamic binding of histone H1 to chromatin in living cells." Nature, 408, 877.

(291) BöHM, L. \& MitchelL, T. C. (1985). FEBS Letters, 193, 1. 
(292) Chapman, G. E.;Hartman, P. G.;CARry, P. D.;Bradbury, E. M. \& LeE, D. R. (1978). European Journal of Biochemistry, 85, 35.

(293) LENNARD, A. C. \& ThOMAS, J. O. (1985). "The arragement of histone H5 molecules in extended and condensed chicken erythrocyte chromatin." EMBO Journal, 4,3455 .

(294) Clark, D. J. \& Kimura, T. (1990). "Electrostatic mechanism of chromatin folding." Journal of Molecular Biology, 211, 883.

(295) WeINTRAUB, H. (1985). "Histone-H1-dependent chromatin superstructures and the suppression of gene activity." Cell, 38, 17.

(296) SMITH, C. L. \& HAGER, G. L. (1997). "Transcriptional regulation of mammalian genes in vivo - a tale of two templates. [Review]." Journal of Biological Chemistry, 272, 27493.

(297) KIM, A. \& DEAN, A. (2003). "A human globin enhancer causes both discrete and widespread alterations in chromatin structure." Molecular \& Cellular Biology, 23, 8099.

(298) Zlatanova, J. \& VAN Holde, K. (1992). "Histone H1 and transcription: still an enigma?" Journal of Cell Science, 103, 889.

(299) Khochbin, S. \& WolfFe, A. P. (1994). "Developmentally regulated expression of linker-histone variants in vertebrates. [Review]." European Journal of Biochemistry, 225, 501 .

(300) Shen, X. T. \& Gorovsky, M. A. (1996). "Linker-histone H1 regulates specific gene expression but not global transcription in vivo." Cell, 86, 475.

(301) BROWN, D. T. (2003). "Histone H1 and the dynamic regulation of chromatin function." Biochemistry \& Cell Biology-Biochimie \& Biologie Cellulaire, 81, 221.

(302) BARra, J. L.;Rhounim, L.;Rossignol, J. L. \& FAugeron, G. (2000). "Histone $\mathrm{H} 1$ is dispensable for methylation-associated gene silencing in Ascobolus immersus and essential for long life span." Molecular \& Cellular Biology, 20, 61.

(303) Downs, J. A.;Kosmidou, E.;Morgan, A. \& Jackson, S. P. (2003).

"Suppression of homologous recombination by the Saccharomyces cerevisiae linkerhistone." Molecular Cell, 11, 1685.

(304) Konishi, A.;SHIMIZU, S. et al. (2003). "Involvement of histone H1.2 in apoptosis induced by DNA double-strand breaks." Cell, 114, 673.

(305) CiRILlo, L. \& ZARET, K. (2004). "A linker-histone restricts muscle development." Science, 304, 1607.

(306) BötTGer, M.;Mickwitz, C.-U. V.;SCHERneCK, S. \& Lindigkeit, R. (1984).

"Interaction of histone $\mathrm{H} 1$ with superhelical DNA - confomartional studies and influence of ionic strength." Molecular Biology Reports, 10, 3. 
(307) Clark, D. J. \& ThOMAS, J. O. (1986). "Salt-dependent cooperative interaction of histone H1 with linear DNA." Journal of Molecular Biology, 187, 569.

(308) Draves, P. H.;LOWARY, P. T. \& WidOM, J. (1992). "Cooperative binding of the globular domain of histone H5 to DNA." Journal of Molecular Biology, 225, 1105.

(309) Ellen, T. P. \& VAN HoldE, K. E. (2004). "Linker-histone interaction shows divalent character with both supercoiled and linear DNA." Biochemistry, 43, 7867.

(310) IvanchenKo, M.;Hassan, A.;VAnholde, K. \& Zlatanova, J. (1996). "H1 binding unwinds DNA - evidence from topological assays." Journal of Biological Chemistry, 271, 32580.

(311) KRYlov, D.;Leuba, S.;VAnholde, K. \& Zlatanova, J. (1993). "Histones H1 and $\mathrm{H} 5$ interact preferentially with crossovers of double-helical DNA." Proceedings of the National Academy of Sciences of the United States of America, 90, 5052.

(312) Thomas, J. O.;REes, C. \& Finch, J. T. (1992). "Cooperative binding of the globular domains of histones H1 and H5 to DNA." Nucleic Acids Research, 20, 187.

(313) Vargaweisz, P.;Zlatanova, J.;Leuba, S. H.;Schroth, G. P. \& VAnhOlde, K. (1994). "Binding of histones H1 and H5 and their globular domains to 4-way junction DNA." Proceedings of the National Academy of Sciences of the United States of America, 91, 3525.

(314) WatanaBE, F. (1986). "Cooperative interaction of histone H1 with DNA." Nucleic Acids Research, 14, 3573.

(315) De NoOiJ, E. H. \& WestenbrinK, H. G. K. (1962). "Isolation of a homogenous lysine-rich histone from calf thymus." Biochimica et Biophysica Acta, 62, 608.

(316) DeRouchey, J.;Netz, R. R. \& RAdLER, J. O. (2005). "Structural investigations of DNA-polycation complexes." European Physical Journal E, 16, 17.

(317) IvanchenKo, M.;Zlatanova, J. \& Vanholde, K. (1997). "Histone H1 preferentially binds to superhelical DNA molecules of higher compaction." Biophysical Journal, 72, 1388.

(318) Mamoon, N. M.;Song, Y. G. \& Wellman, S. E. (2005). "Binding of histone H1 to DNA is described by an allosteric model." Biopolymers, 77, 9.

(319) Morán, F.;MOnTERo, F.;Azorín, F. \& SuAU, P. (1985). "Condensation of DNA by the C-terminal domain of histone H1. A circular dichroism study." Biophys. Chem., 22, 125.

(320) Fasman, G. D.;Schaffhausen, B.;Goldsmith, L. \& Adler, A. (1970). "Conformational chages associated with $\mathrm{f}-1$ histone deoxyribonucleic acid complex. Circular dichroism studies." Biochemistry, 9, 2814. 
(321) BHARATH, M. M. S.;CHANDRA, N. R. \& RAO, M. R. S. (2002). "Prediction of an HMG-box fold in the C-terminal domain of histone H1: Insights into its role in DNA condensation." Proteins: Structure, Function, \& Genetics, 49, 71.

(322) Hardman, C. H.;BRoAdhurst, R. W. et al. (1995). "Structure of the A-domain of HMG1 and its interaction with DNA as studied by heteronuclear three- and fourdimensional NMR spectroscopy." Biochemistry, 34, 16596.

(323) Love, J. J.;LI, X. A. et al. (1995). "Structural basis for DNA bending by the architectural transcription factor Lef-1." Nature, 376, 791.

(324) Werner, M. H.;Ruth, J. R.;Gronenborn, A. M. \& Clore, G. M. (1995). "Molecular basis of human 46x, Y sex reversal revealed from the three-dimensional solution structure of the human Sry-DNA complex." Cell, 81, 705.

(325) Sheflin, L. G.;Fucile, N. W. \& SPAulding, S. W. (1993). "The specific interactions of HMG1 and 2 with negatively supercoiled DNA are modulated by their acidic C-terminal domains and involve cysteine residues in their HMG 1/2 boxes." Biochemistry, 32, 3238. 


\subsection{Publications}

"Highly packed and oriented DNA mesophases identified using in situ microfluidic X-ray microdiffraction."

Thomas Pfohl, Alexander Otten, Sarah Köster, Rolf Dootz, Bernd Struth, and Heather M. Evans (2007). Biomacromolecules, 8 (7), 2167-2172. doi:10.1021/bm070317s

"X-ray microdiffraction on flow-controlled biomolecular assemblies."

Heather M Evans, Rolf Dootz, Sarah Köster, Bernd Struth, and Thomas Pfohl (2007).

Bulletin of the Polish Academy of Sciences: Technical Sciences 55 (2), 217-227.

„Rapid prototyping of X-ray microdiffraction compatible continuous microflow foils.“

Rolf Dootz, Heather Evans, Sarah Köster, and Thomas Pfohl (2007). Small 3 (1), 96-100. $\underline{\text { doi: } 10.1002 / \mathrm{smll} .200600288}$

"Evolution of DNA compaction in microchannels."

Rolf Dootz, Alexander Otten, Sarah Köster, Bernd Struth, and Thomas Pfohl (2006). J. Phys.:

Condensed Matter, 18, S639-S653. doi:10.1088/0953-8984/18/18/S10

"Raman and surface enhanced Raman microscopy of microstructured PEI/DNA multilayers." Rolf Dootz, Jingjing Nie, Binyang Du, Stephan Herminghaus, and Thomas Pfoh (2006) Langmuir 22, 1735-1742. doi:10.1021/la052739y

"Time-resolved X-ray microdiffraction on linker-histone-DNA interaction."

Rolf Dootz and Thomas Pfohl. In preparation.

"Demonstrating fundamental principles of microfluidics using linker-histone induced DNA compaction."

Rolf Dootz and Thomas Pfohl. In preparation.

"Charge induced changes in dendrimer conformation."

Rolf Dootz and Thomas Pfohl. In preparation.

"Complex structural organization of PAMAM 6/DNA condesates."

Rolf Dootz and Thomas Pfohl. In preparation.

"Formation of artificial nucleosome core particles."

Rolf Dootz and Thomas Pfohl. In preparation. 


\section{DANKSAGUNG}

Abschließend möchte ich mich bei allen Personen bedanken, die zum Gelingen dieser Arbeit beigetragen haben.

Allen voran gebührt mein herzlicher Dank Thomas Pfohl für seine hervorragende und engagierte Betreuung. Für mich war Thomas während der gesamten Arbeit der ideale Betreuer, der immer das richtige Mittelmaß zwischen intensiver Betreuung und notwendigem Freiraum fand. Ich konnte mich in fachlicher und persönlicher Hinsicht immer auf seine Hilfe und sein Verständnis verlassen. Sein fortwährendes Interesse an meiner Arbeit sowie die stete Bereitschaft zur Diskussion bildeten die wissenschaftliche Grundlage für ihre Anfertigung.

Mein besonderer Dank gilt auch Stephan Herminghaus, der mir die Möglichkeit gab, meine Arbeit am MPI für Dynamik und Selbstorganisation durchzuführen und die tolle Ausstattung des Institutes zu nutzen. Er stand mir stets mit ausführlichen Diskussionen, wertvollen Anregungen sowie großzügiger und uneingeschränkter Unterstützung zur Seite. Tim Salditt danke ich ganz herzlich für die Übernahme des Koreferats. Darüber hinaus bin ich Tim für sein Interesse an meinem Projekt, für seine Einladung zur Winterschule und insbesondere für seine ansteckende Begeisterung dankbar. Weiteren Dank schulde ich Erwin Neher, Carsten Ronning, Detlev Schild und Rainer Ulbrich, die sich spontan und bereitwillig als Prüfer zur Verfügung stellten und bei der Absprache von Prüfungsfächern und -terminen sehr flexibel waren.

Der gesamten Arbeitsgruppe Pfohl bin ich nicht nur für die Unterstützung beim Gelingen meines Projektes sondern auch für die angenehme, motivierende und freundschaftliche Atmosphäre und den tollen Zusammenhalt dankbar. Ohne Heather Evans, Sarah Köster, Semra Öztürk, Dagmar Steinhauser, Eric Stellamanns und Sravanti Uppaluri wäre manche Stunde langweiliger und fruchtloser gewesen. Heather bin ich besonders für die Durchsicht einiger Teile meiner Arbeit dankbar. Die eine oder andere geschliffene Wendung geht sicherlich auf sie zurück. Semra schulde ich außerdem Dank für ihre tatkräftige und vorbildliche Organisation des Laborbetriebs und für die Abwicklung meiner Bestellungen. Außerdem möchte ich Alexander Otten für die Aufnahme wertvoller experimenteller Daten danken, die in Kapitel 7.2 eingeflossen sind und die mir eine vollständige Auswertung erst möglich machten. 
Meine Arbeit profitierte von fruchtbaren Gesprächen mit einer großen Zahl von Leuten. Insbesondere möchte ich dabei Martin Brinkmann erwähnen, dem ich für die hilfreichen, fachlichen Diskussionen dankbar bin, die den einen oder anderen Fehler frühzeitig zu erkennen und zu beseitigen halfen.

Ohne hervorragende technische Unterstützung wäre diese Arbeit nicht möglich gewesen. Mein Dank geht deswegen an Udo Krafft, der sich für alle meine sinnigen und unsinnigen Ideen Zeit genommen hat und manchmal davon sogar noch begeisterter war als ich selbst. Ohne seine unkonventionellen Lösungen für viele technische Probleme wäre diese Arbeit in der vorliegenden Form nicht möglich gewesen. Des Weiteren war ich immer sehr dankbar, in Wolf Keiderling einen kompetenten und hilfsbereiten Ansprechpartner für technische Probleme jeder Art zu haben. Ohne die Unterstützung von Bernd Struth, Oleg Konovalov und Anatoly Snigirev wären die Experimente am ESRF in Grenoble sicherlich nicht möglich gewesen. Marcus Schwamberger danke ich für seine fachkundige Unterstützung bei allen kleineren und größeren Computerproblemen. Monika Teuteberg danke ich für ihren hervorragenden organisatorischen Beistand sowie für die prompte Hilfe bei Druckerproblemen.

Außerdem möchte ich auch allen Teilnehmern des Mittagssports für ihre tatkräftige Unterstützung beim Einlegen spontaner Erholungspausen danken. Sie waren häufig das nötige Ventil für Freud und Leid und das probate Mittel, um aus Motivationslöchern wieder rauszukommen. Mein Dank gilt auch einem gewissen roten Gerät, ohne welches die hier vorliegende Arbeit niemals so schnell fertig geworden wäre.

Abschließend möchte ich meiner Familie danken. Ohne ihre kontinuierliche Unterstützung wäre ich nicht in der Lage gewesen, diesen Weg zu gehen. Meine Eltern haben mich auf meinem bisherigen Lebensweg uneingeschränkt unterstützt. Ihre moralische und finanzielle Unterstützung haben den Grundstein zum Gelingen dieser Arbeit gelegt und können nicht hoch genug bemessen werden. Dafür meinen Dank. Meinen Schwiegereltern möchte ich dafür danken, dass sie immer ein offenes Ohr für meine Sorgen hatten. Ich konnte und kann mich immer auf sie verlassen.

Während meiner Promotionszeit habe ich mich nicht selten so gefühlt, als würde ich am Abgrund stehen. Dass ich nicht abgestürzt bin verdanke ich vor allem meiner Frau Tanja. Klaglos hat sie alle meine Launen ertragen, meinen Monologen über meine Arbeit gelauscht und mich durch alle Höhen und Tiefen der Arbeit begleitet. Ihre liebevolle Unterstützung, ihre Geduld, ihr Verständnis und ihre Aufmunterungen waren und sind mir stets ein Rückhalt. Deshalb widme ich ihr diese Arbeit. 


\section{CURRICULUM VITAE}

Persönliche Angaben

Nachname: Dootz

Vorname: $\quad$ Rolf

Geburtstag: $\quad$ 19. Mai 1978

Geburtsort: $\quad$ Kronstadt/Rumänien

Staatsangehörigkeit: deutsch

Ausbildung

Seit Aug. 2004

Promotionsstudium am Max-Planck-Institut für Dynamik und Selbstorganisation in Göttingen

08. Juli 2004

Abschluss des Diplom-Studiengangs Physik

Febr. 2003- Febr. 2004

Diplomarbeit in der Abt. Angewandte Physik, Universität Ulm

Okt. 1998 - Juli 2004

Diplomstudium der Physik an der Universität Ulm

Juli 1997 - Aug. 1998

Zivildienst

Juni 1989 - Juli 1997

Hellenstein-Gymnasium in Heidenheim, Abschluss: Abitur

1988 - April 1989

Johannes-Honterus-Gymnasium in Kronstadt 